\title{
Production planning for flexible manufacturing systems
}

Citation for published version (APA):

Oerlemans, A. G. (1992). Production planning for flexible manufacturing systems. [Doctoral Thesis, Maastricht University]. Datawyse / Universitaire Pers Maastricht. https://doi.org/10.26481/dis.19921009ao

Document status and date:

Published: 01/01/1992

DOI:

10.26481/dis.19921009ao

Document Version:

Publisher's PDF, also known as Version of record

\section{Please check the document version of this publication:}

- A submitted manuscript is the version of the article upon submission and before peer-review. There can be important differences between the submitted version and the official published version of record.

People interested in the research are advised to contact the author for the final version of the publication, or visit the DOI to the publisher's website.

- The final author version and the galley proof are versions of the publication after peer review.

- The final published version features the final layout of the paper including the volume, issue and page numbers.

Link to publication

\footnotetext{
General rights rights.

- You may freely distribute the URL identifying the publication in the public portal. please follow below link for the End User Agreement:

www.umlib.nl/taverne-license

Take down policy

If you believe that this document breaches copyright please contact us at:

repository@maastrichtuniversity.nl

providing details and we will investigate your claim.
}

Copyright and moral rights for the publications made accessible in the public portal are retained by the authors and/or other copyright owners and it is a condition of accessing publications that users recognise and abide by the legal requirements associated with these

- Users may download and print one copy of any publication from the public portal for the purpose of private study or research.

- You may not further distribute the material or use it for any profit-making activity or commercial gain

If the publication is distributed under the terms of Article $25 \mathrm{fa}$ of the Dutch Copyright Act, indicated by the "Taverne" license above, 


\section{PRODUCTION PLANNING FOR FLEXIBLE MANUFACTURING SYSTEMS}




\title{
PRODUCTION PLANNING FOR FLEXIBLE MANUFACTURING SYSTEMS
}

\author{
PROEFSCHRIFT
}

\author{
ter verkrijging van de graad van doctor \\ aan de Rijksuniversiteit Limburg te Maastricht, \\ op gezag van de Rector Magnificus, Prof. Mr. M.J. Cohen, \\ volgens het besluit van het College van Dekanen, \\ in het openbaar te verdedigen op \\ vrijdag, 9 oktober 1992 om 14.00 uur
}

door

Alwin Gerolt Oerlemans

geboren te Utrecht 


\section{Promotor:}

Prof.dr.ir. A.W.J. Kolen

\section{Co-promotor:}

Dr. Y. Crama

\section{Beoordelingscommissie:}

Prof.dr.ir.drs. O.J. Vrieze (voorzitter)

Prof.dr. G. Finke (Université Joseph Fourier, Grenoble)

Prof.dr. H.Th. Jongen (Rheinisch-Westfälische Technische Hochschule, Aachen)

Dr. H.J.M. Peters

Prof.dr. W.H.M. Zijm (Universiteit Twente, Enschede)

\section{CIP-DATA KONINKLIJKE BIBLIOTHEEK, DEN HAAG}

\section{Oerlemans, Alwin Gerolt}

Production planning for flexible manufacturing systems / Alwin Gerolt Oerlemans. - Maastricht : Universitaire Pers Maastricht. - III.

Thesis Maastricht. - With index, ref. - With summary in Dutch.

ISBN 90-5278-035-8

NUGI 811/849

Subject headings: production planning / tool management I flexible manufacturing systems.

() 1992 A.G. Oerlemans, Maastricht 
Aan mijn ouders en Simone 


\section{Preface}

The work described in this thesis started in the spring of 1988. At that time I had just completed my Master's thesis in Operations Research and Econometrics at the Erasmus University in Rotterdam and I barely knew anything about flexible manufacturing systems (FMS). It was my thesis advisor Antoon Kolen who introduced me to this new field of application for techniques from Operations Research. This was the starting point of our cooperation in the last 4 years of which this thesis is the endproduct.

My decision to come to Maastricht proved to be the right one and I owe a lot to the group of researchers I worked with at the University of Limburg. The research environment at the University of Limburg is international, informal and innovative. Also, the beauty and style of Maastricht and South-Limburg offer countless opportunities for enjoying a Burgundian life every now and then. I have learned a lot on the application of flexible manufacturing technology and the development of models to improve the efficiency of these systems during the last years. I have to thank my thesis advisors Yves Crama and Antoon Kolen for the many hours we spent discussing planning and combinatorial optimization problems and the patience and expertise with which they guided this research.

The leitmotiv in the first part of the journey into the field of flexible manufacturing were the frequent meetings together with Frits Spieksma and our advisors Yves Crama and Antoon Kolen. The research described in Chapters 2 and 6 of this thesis originated at these meetings. In Chapter 2 a real-world production planning problem in the automated manufacturing of printed circuit boards is described. Under the difection of Yves Crama and Antoon Kolen, Frits Spieksma and I worked on this project. This research was followed by a another joint project concentrating on recognizing combinatorial properties and developing solution strategies for a specific problem occurring in FMS production planning, i.e. the problem of minimizing the number of tool switches, which is described in Chapter 6. 
The topic of efficient tool management got a hold of us and other results of work in this field are described in Chapters 3, 4 and 5. I conducted the research described in these chapters under the guidance of Yves Crama. It was during this period that the final shape of this thesis was decided on and without the great interest and important comments of Yves Crama this thesis would not have had its final form. I thank Yves Crama for his advice and encouragement and Antoon Kolen for directing the work for the thesis. Special thanks goes to Henk $\mathrm{Zijm}$ for introducing us to the production planning problem, discussed in Chapter 2. I also want to thank Erwin Pesch for his interest in the project during this research.

My stay at the University of Limburg would not have been so enjoyable without many others which contributed to my work and well-being. First I would like to thank Karin van den Boorn, Ellen Nelissen and Yolanda Paulissen for their role in taking care of the social activities in the department, but also for their generous secretarial support during these years. I would like to thank my roommates. First, I thank Frits Spieksma for our fruitful cooperation. I thank Hugo Kruiniger for his interest in other topics than his own research and the many discussions we had on nearly every topic in the political landscape. Special thanks go to Marcel Lever and Hans Voordijk for their interest in the progress of this research and the many meals we shared discussing other aspects of life during the last years.

At this point I want to express my gratitude to professors Gerd Finke, Bert Jongen, Hans Peters, Koos Vrieze and Henk Zijm for being part of the reading committee.

I also want to thank the members of the Faculty of Economics and Business Administration, the Department of Mathematics and others who contributed to this research in one way or another, especially Peter van Laarhoven, Joe Mazzola, Maarten Oosten, Marga Peeters, Gerard Pfann, François Soumis, Peter Vlaar and Ron van der Wal.

I want to thank the University of Limburg for the opportunity they gave me to do research and visit conferences in Europe and North-America. My gratitude also goes to the Rutgers Center for Operations Research (New Brunswick, NJ), Shell Nederland B.V. and the Stichting Wetenschappelijk Onderzoek Limburg for (travel) grants I received during the last years.

Finally, I would like to thank my parents for stimulating me to explore new opportunities and Simone Kaars for her support and for abandoning many weekends during the last year(s).

Maastricht, May 1992. 


\section{Contents}

\section{Chapter 1}

Flexible manufacturing: concepts and models $\ldots \ldots \ldots \ldots \ldots$

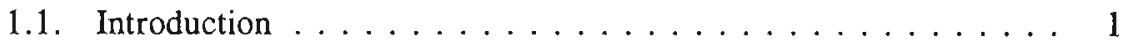

1.2. Automated manufacturing $\ldots \ldots \ldots \ldots \ldots \ldots$

1.2.1. What is an FMS? . . . . . . . . . . . . 3

1.2.2. Classification and diffusion of FMS ........ 4

1.2.3. Must FMS be justified by faith alone? . . . . . . . . 7

1.3. Hierarchical approaches to FMS production planning . . . . . . 9

1.3.1. Strategic planning . . . . . . . . . . . 11

1.3.2. Tactical planning . . . . . . . . . . . . 12

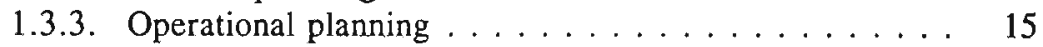

1.4. Overview of the thesis $\ldots \ldots \ldots \ldots \ldots \ldots$

\section{Chapter 2}

Throughput rate optimization in the automated assembly of printed circuit boards . . . . . . . . . . . . 21

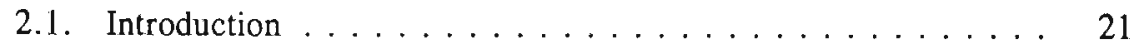

2.2. Technological environment .............. 22

2.3. The throughput rate optimization problem . . . . . . . 25

2.4. Workload balancing .................. . 27

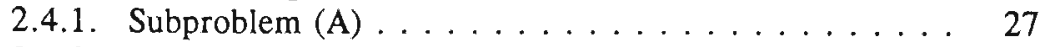

2.4.2. Subproblem (B) ............... 31 
2.5. Scheduling of individual machines ... . . . . . . 34

2.5.1. Subproblem (C) . . . . . . . . . . . . . . 34

2.5.2. Subproblem (D) $\ldots \ldots \ldots \ldots \ldots \ldots \ldots$

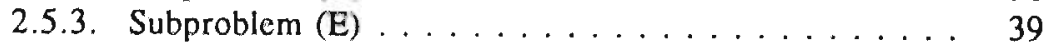

2.5.4. Subproblem $(\mathrm{F}) \ldots \ldots \ldots \ldots \ldots \ldots \ldots \ldots$

2.6. An example ........................ 44

2.6.1. Subproblem (A) . . . . . . . . . . . . 45

2.6.2. Subproblem (B) . . . . . . . . . . . . . 46

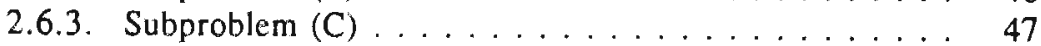

2.6.4. Subproblem (D) . . . . . . . . . . . . . . 47

2.6.5. Subproblem (E) and $(F) \ldots \ldots \ldots \ldots$

Chapter 3

A column generation approach to job grouping for

flexible manufacturing systems . . . . . . . . . . . . . . . 49

3.1. Introduction . . . . . . . . . . . . . . . . . 49

3.2. Lower bounds . . . . . . . . . . . . . . 51

3.2.1. The job grouping problem ........... 51

3.2.2. Column generation ................ 53

3.2.3. The generation subproblem . . . . . . . . . . 54

3.2.4. Computation of lower bounds via column generation . . 57

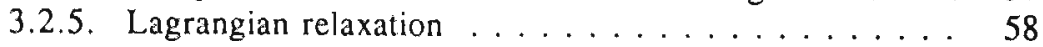

3.2.6. Other lower bounds ................ . 61

3.3. Upper bounds . . . . . . . . . . . . . . . . 63

3.3.1. Sequential heuristics for grouping .......... 63

3.3.2. Set covering heuristics . . . . . . . . . . . . . . 64

3.4. Implementation . . . . . . . . . . . . . . 65

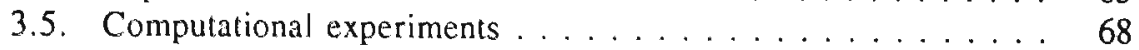

3.5.1. Generation of problem instances . . . . . . . . . 68

3.5.2. Computational results . . . . . . . . . . . 70

3.6 Summary and conclusions $\ldots \ldots \ldots \ldots \ldots \ldots$

\section{Chapter 4}

The job grouping problem for flexible manufacturing systems: some extensions

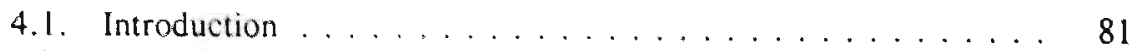

4.2. Multiple slots . . . . . . . . . . . . . . 81

4.2.1. The job grouping problem . . . . . . . . 81

4.2.2. Lower bounds via column generation . . . . . . . 83 
4.2.3. Other lower bounds . . . . . . . . . . . 85

4.2.4. Upper bounds . . . . . . . . . . . . . 85

4.2.5. Adjusting the column generation procedure . . . . . 87

4.2.6. Computational experiments . . . . . . . . . . 88

4.2.7. Computational results ................ 9

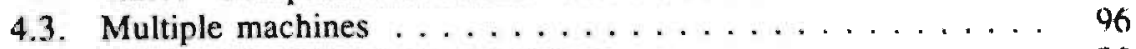

4.3.1. The job grouping problem . . . . . . . . . . 96

4.3.2. Lower bounds via column generation . . . . . . . . . 97

4.3.3. Other lower bounds . . . . . . . . . . . . . . 98

4.3.4. Upper bounds . . . . . . . . . . . . . . . . 99

4.3.5. Adjusting the column generation procedure . . . . . . . 99

4.3.6. Computational experiments . . . . . . . . . . . . 99

4.3.7. Computational results . . . . . . . . . . 100

4.4. Other extensions ................... . . 105

4.5. Summary and conclusions . . . . . . . . . . 106

\section{Chapter 5}

A local search approach to job grouping . . . . . . . . . 107

5.1. Introduction . . . . . . . . . . . . . . . . . 107

5.2. Local search environment . . . . . . . . . . . . . 108

5.2.1. Starting solution ................. 109

5.2.2. Objective function $\ldots \ldots \ldots \ldots \ldots \ldots \ldots$

5.2.3. Neighbourhood structure . . . . . . . . . 111

5.2.4. Stopping criteria .............. 111

5.3. Local search approaches ................. 112

5.3.1. Simple improvement approach ........... 112

5.3.2. Tabu search approach .............. 112

5.3.3. Simulated annealing approach .......... 113

5.3.4. Variable-depth approach ............ 114

5.4. Computational experiments . . . . . . . . . . . 114

5.4.1. The dataset ................... . . 115

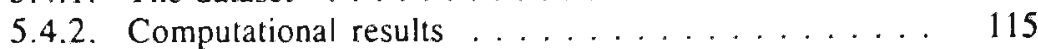

5.5. Summary and conclusions . . . . . . . . . . 122 
2.5. Scheduling of individual machines . . . . . . . . 34

2.5.1. Subproblem (C) . . . . . . . . . . . . . . 34

2.5.2. Subproblem (D) $\ldots \ldots \ldots \ldots \ldots \ldots$

2.5.3. Subproblem (E) . . . . . . . . . . . . . . . 39

2.5.4. Subproblem (F) . . . . . . . . . . . . . . 42

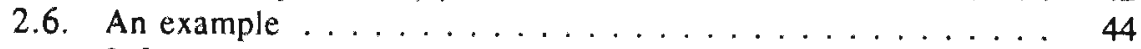

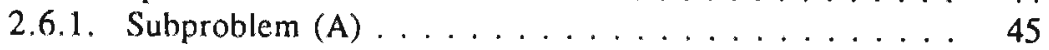

2.6.2. Subproblem (B) . . . . . . . . . . . . 46

2.6.3. Subproblem (C) . . . . . . . . . . . . . . 47

2.6.4. Subproblem (D) . . . . . . . . . . . . . . 47

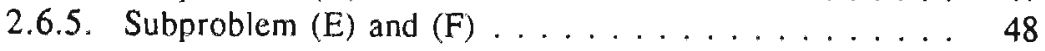

\section{Chapter 3}

A column generation approach to job grouping for

flexible manufacturing systems

3.1. Introduction . . . . . . . . . . . . . . . 49

3.2. Lower bounds . . . . . . . . . . . . . . . 51

3.2.1. The job grouping problem $\ldots \ldots \ldots \ldots \ldots \ldots$

3.2.2. Column generation ............... 53

3.2.3. The generation subproblem . . . . . . . . . . 54

3.2.4. Computation of lower bounds via column generation . . 57

3.2.5. Lagrangian relaxation . . . . . . . . . . . 58

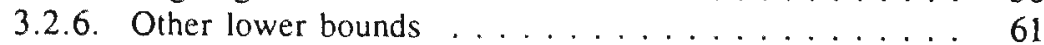

3.3. Upper bounds . . . . . . . . . . . . . . . . 63

3.3.1. Sequential heuristics for grouping .......... 63

3.3.2. Set covering heuristics . . . . . . . . . . . . . 64

3.4. Implementation . . . . . . . . . . . . . . 65

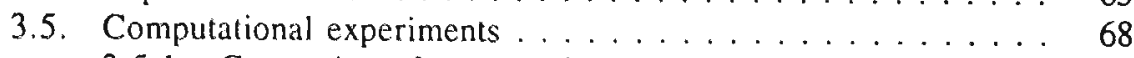

3.5.1. Generation of problem instances . . . . . . . . . 68

3.5.2. Computational results . . . . . . . . . . . . 70

3.6 Summary and conclusions . . . . . . . . . . . . 79

\section{Chapter 4}

The job grouping problem for flexible manufacturing systems:

some extensions

4.1. Introduction . . . . . . . . . . . . . . 81

4.2. Multiple slots . . . . . . . . . . . . . 81

4.2.1. The job grouping problem . . . . . . . . . . 81

4.2.2. Lower bounds via column generation . . . . . . . 83 
4.2.3. Other lower bounds . . . . . . . . . . . . . 85

4.2.4. Upper bounds . . . . . . . . . . . . . . 85

4.2.5. Adjusting the column generation procedure . . . . . 87

4.2.6. Computational experiments . . . . . . . . . . 88

4.2.7. Computational results . . . . . . . . . . . . . 90

4.3. Multiple machines . . . . . . . . . . . . . 96

4.3.1. The job grouping problem . . . . . . . . . . . 96

4.3.2. Lower bounds via column generation . . . . . . . . . 97

4.3.3. Other lower bounds . . . . . . . . . . . . . . . . 98

4.3.4. Upper bounds . . . . . . . . . . . . . . . . . . . . 99

4.3.5. Adjusting the column generation procedure . . . . . . 99

4.3.6. Computational experiments ............... . 99

4.3.7. Computational results ............. 100

4.4. Other extensions . . . . . . . . . . . . . 105

4.5. Summary and conclusions . . . . . . . . . . . 106

\section{Chapter 5}

A local search approach to job grouping . . . . . . . . . . 107

5.1. Introduction . . . . . . . . . . . . . . . . . . . 107

5.2. Local search environment ................. 108

5.2.1. Starting solution . . . . . . . . . . . . . 109

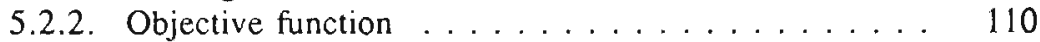

5.2.3. Neighbourhood structure . . . . . . . . . . 111

5.2.4. Stopping criteria . . . . . . . . . . . . 111

5.3. Local search approaches ... . . . . . . . . . . . . 112

5.3.1. Simple improvement approach ........... 112

5.3.2. Tabu search approach . . . . . . . . . . . 112

5.3.3. Simulated annealing approach ........... 113

5.3.4. Variable-depth approach ............. 114

5.4. Computational experiments . . . . . . . . . . . . . 114

5.4.1. The dataset . . . . . . . . . . . . . . . . . 115

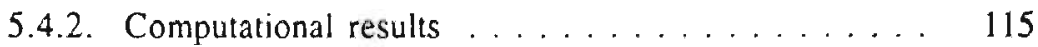

5.5. Summary and conclusions . . . . . . . . . . . . 122 


\section{Chapter 6}

Minimizing the number of tool switches on a flexible machine . . . 125

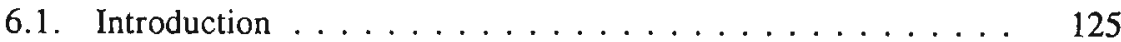

6.2. Basic results . . . . . . . . . . . . . . . . . . . . . 129

6.2.1. NP-hardness results . . . . . . . . . . . . 129

6.2.2. Finding the minimum number of setups for a fixed job sequence . . . . . . . . . . . . . . 130

6.3. Heuristics . . . . . . . . . . . . . . . . . . . . 134

6.3.1. Traveling salesman heuristics . . . . . . . 135

6.3.2. Block minimization heuristics ... . . . . . . 136

6.3.3. Greedy heuristics . . . . . . . . . . . . . . 137

6.3.4. Interval heuristic . . . . . . . . . . . . . 138

6.3.5. 2-Opt strategies . . . . . . . . . . . . . . . . 139

6.3.6. Load-and-Optimize strategy . . . . . . . . . . 140

6.4. Computational experiments . . . . . . . . . . . . . . . 141

6.4.1. Generation of problem instances . . . . . . . . . . 141

6.4.2. Computational results . . . . . . . . . . . . 143

6.5. Lower bounds . . . . . . . . . . . . . . . . . . . . 147

6.5.1. Traveling salesman paths ............. 147

6.5.2. Structures implying extra setups . . . . . . . . . 149

6.5.3. Valid inequalities ................. 151

6.5.4. Lagrangian relaxation . . . . . . . . . . . . . 153

6.6. Summary and conclusions . . . . . . . . . . . . 153

Appendix: Graph-theoretic definitions $\ldots \ldots \ldots \ldots \ldots \ldots$

References . . . . . . . . . . . . . . . . . 157

Author index . . . . . . . . . . . . . . . . . . . . . . . . 169

Samenvatting $\ldots \ldots \ldots \ldots \ldots \ldots \ldots \ldots \ldots \ldots$

Curriculum vitae $\ldots \ldots \ldots \ldots \ldots \ldots \ldots \ldots \ldots \ldots$ 


\section{Chapter 1}

\section{Flexible manufacturing: concepts and models}

\subsection{Introduction}

In the last two decades we have witnessed great changes in manufacturing technology. Increased automation has cleared the way for what some people call the "factory of the future". However, this factory already exists today. It distinguishes itself from conventional factories by a high degree of automation, reduced use of labour and raw materials and a high degree of flexibility to respond quickly to market changes. The subject of this thesis is the development of quantitative methods to help solve problems related to the planning of automated manufacturing systems.

The first chapter is devoted to a presentation of the subject of automated manufacturing. Its developments and benefits are presented. Also, an introduction is given on the efforts of the operations research community to provide quantitative support for the design, implementation and operation of these highly automated systems. Attention is focused on the use of deterministic models. The last section (Section 1.4) of this chapter gives an outline of the work described in the remainder of this thesis.

\subsection{Automated manufacturing}

Manufacturers around the world are confronted with rapid changes in market demand. The ability to respond quickly to these changes is a key to survive in global competition. However, this requires the production 
philosophy to change dramatically. Not only the layout and design of factories, but also the organization and development of new products has to change (Cook, 1975; Zijm, 1988).

The developments in information-intensive processing technologies have only increased the pace of these changes on the factory floor. Automation in manufacturing has been an important factor in achieving changes in the factory. A wide body of literature on manufacturing automation has emerged in the last decade in technical, management and mathematically oriented journals. Concepts relating to automated manufacturing are e.g. Computer Aided Design (CAD), Computer Aided Manufacturing (CAM), robotics, Flexible Manufacturing Systems (FMS) and Computer Integrated Manufacturing (CIM). The introduction of automated manufacturing and increased availability of information technology also offered new tools for management to control the flow of goods in the factory. Especially in the last decades we have seen the rise of methods like Materials Requirements / Manufacturing Resources Planning (MRP), Optimized Production Technology (OPT) and the implementation of Just-In-Time (JIT) principles on the factory floor (see Aggarwal (1985)).

A starting point in the development of automated manufacturing was the use of numerically controlled (NC) machine tools in the 1950's. NCtechnology permits complex parts to be manufactured rapidly and accurately by automated machines. NC-machines use a numerical control method for activating the tools in response to a predetermined command stored on punch (or magnetic) tapes. A further development was the introduction of computer numerical control (CNC) in which a mini- or micro-computer is used to control the NC machine tools. Since the late 1960's CNC has taken over the major part of the NC machine tool industry. If a group of $\mathrm{NC}$ (or $\mathrm{CNC}$ ) machines is simultaneously controlled by one host computer, we speak of direct numerical control (DNC).

Computer Aided Design (CAD) has emerged since the 1960's as a flexible method to design products and equipments. CAD coupled with NC manufacturing is called Computer Aided Manufacturing. The increased availability of cheap computers also dramatically changed the way production was organized and controlled. Manufacturing Resource Planning (MRP) is one of these methods. The emergence of $C A D / C A M$ and adequate production planning provided an opportunity for improving the productivity of the design and manufacturing process (see also Groover and Zimmers (1984)). 
The current developments head in the direction of the totally automated factory, where raw materials are processed into finished goods without direct human intervention. This way of producing is also called Computer Integrated Manufacturing (CIM). CIM requires that material handling, assembly, inspection and design be fully integrated. Human involvement concentrates on control, maintenance and design. A growing number of systems which fit this description are used around the world.

In some industries automated manufacturing has a long history, especially in chemicals or oil refining (Dorf, 1983). However, in the batchmanufacturing industries, such as metal working, it has been introduced only in the 1970's. Our focus will be on this type of industry. Flexible manufacturing systems are a critical component for the introduction of the so-called "factory of the future" in the batch-manufacturing industries. Jaikumar (1986) points out that this new technology has drastically changed the competitive landscape. A close attention to the market and regular adjustments of price and product-mix must guarantee full capacity utilization. Fixed cost have to be reduced and the development time of new products has to be decreased. To achieve the benefits of FMSs, teams must be created to work on the manufacturing projects. The intellectual development of the employees must be stimulated. Jaikumar (1986) also stresses the integration of design and manufacturing and a focus on process improvement.

The rest of this section will be devoted to the description, the history and diffusion and the economic benefits of FMSs.

\subsubsection{What is an FMS?}

A flexible manufacturing system is an integrated, computer-controlled complex of automated material handling devices and numerically controlled machines that can simultaneously process medium-sized volumes of a variety of part types (Stecke, 1983). As Huang and Chen (1986) and Gerwin (1982) point out, FMSs are an attempt to solve the production problem of midvolume (200-20,000 parts per year) and midvariety parts, for which neither the high-production rate transfer lines nor the highly flexible stand-alone NC machines are suitable. The aim is to achieve the efficiency of mass-production, while utilizing the flexibility of manual job shop production.

An FMS consists of a number of machines or work stations that are used to perform operations on parts. Each operation requires a number of tools, 
that can be stored in the limited capacity tool magazine of the machines. An automatic tool interchanging device quickly interchanges the tools during production. This rapid interchange facility enables the machine to perform several operations with virtually no setup time between operations. Parts are moved automatically to the machines by a transportation system or a Material Handling System (MHS). A number of buffer places or an Automated Storage and Retrieval System (ASRS) are also added to the system, either at a central location or at each machine. In some FMSs, tools are also stored at a central tool store and delivered to machines by a special delivery system (Buzacott and Yao, 1986). Finally, a network of supervisory computers takes care of the control of tools, parts, MHS and machines. The development of FMSs goes along with the other developments in automated manufacturing. The first systems appeared in the 1960's; one of the earliest FMSs, which was designed to process constant speed drive housings for aircraft, was installed by Sunstrand in 1964 (Huang and Chen, 1986). In the late 1970's more systems were developed, while the last decade was mainly devoted to refinement of the systems. Emphasis has shifted from hardware issues to the development of control systems and refinement of the software packages (Huang and Chen, 1986). A number of authors have written excellent books in which detailed descriptions of FMSs are given (Ránky, 1983; Charles Stark Draper Laboratory, 1984; Hartley, 1984; Warnecke and Steinhilper, 1985).

\subsubsection{Classification and diffusion of FMS}

A number of surveys have been published on FMSs that are operational around the world. Several authors have given a classification of FMSs in their surveys.

Groover (1980) partitions FMSs in the following two classes:

(1) Dedicated FMS, which manufacture a fixed set of parts with welldefined manufacturing requirements over a fixed time horizon.

(2) Random FMS that produce a wide variety of parts in random order.

Dupont-Gatelmand (1982) gives a survey (with many illustrations) of several early FMSs in Europe, Japan and the U.S. She divides the systems in three main categories according to the material handling system used:

(1) Flexible modules and units, which consist of a workstation with an automatic parts input/output system;

(2) Flexible conveyor lines, where the flexible units are connected by an automated conveyor system;

(3) Unaligned flexible systems, where the roller conveyor is replaced by Automated Guided Vehicles (AGVs). 
Browne, Dubois, Rathmill, Sethi and Stecke (1984) define eight types of flexibility to clarify the confusion which often occurs when discussing 'flexible' manufacturing systems. They define the following eight types of flexibility:

(1) Machine flexibility: the ease of making changes required to produce a given set of part types.

(2) Process flexibility: the ability to produce a given set of part types, each possibly using different materials in several ways.

(3) Product flexibility: the ability to change over to produce a new (set of) product(s) very economically and quickly.

(4) Routing flexibility: the ability to handle breakdowns and to continue producing the given set of part types.

(5) Volume flexibility: the ability to operate profitably at different production volumes.

(6) Expansion flexibility: the ability to expand the system easily, modularly.

(7) Operation flexibility: the ability to interchange the ordering of several operations for each part type.

(8) Production flexibility: the universe of part types that can be produced.

Sethi and Sethi (1990) extend this list by the following flexibilities:

(9) Material handling flexibility: the ability to move different part types efficiently for proper positioning and processing through the facility served by the MHS.

(10) Market flexibility: the ease with which the manufacturing system can adapt to a changing market environment.

The different flexibility types are not independent of each other. For an FMS, the machine and routing flexibilities are basic flexibilities relating to the CNC machines and the MHS. Other flexibilities depend on these. For a thorough discussion on this topic we refer to the surveys of Gupta and Goyal (1989) and Sethi and Sethi (1990).

This classification can help to categorize FMSs according to their overall flexibility. In Browne et al. (1984) four types of FMSs are described based on flexibility and layout-features such as machine tools, MHS (including tool transportation system), storage areas for in-process inventory and computer control:

I Flexible Machining Cell,

II Flexible Manufacturing System,

III Flexible Transfer Line,

IV Flexible Transfer Multi-Line. 
Type I consists of a single general purpose CNC machine, interfaced with an MHS. Linking different type I systems together with a flexible MHS consisting of shuttle conveyors or AGVs will give a system of type II in which parts are allowed to take several routings to different machines. In a type III system a conveyor line is used as an MHS and each operation is assigned and performed on one specified machine. Each part follows a fixed route through the system. In Type IV combining a number of type III systems offers some additional routing flexibility. Browne et al. (1984) remark that most FMSs in Germany and the U.S. tend to be of Type II, while most systems in Japan are of Type III. This classification can also be used for flexible assembly systems (FAS). In a related study Stecke and Browne (1985) discuss different types of MHS. They discuss the flexibility of belt conveyors, powered roller conveyors, power-and-free conveyors, monotractors or monorails, towlines and automated guided vehicles (mentioned in order of increasing flexibility). The flexibility of the four types of FMSs (see Browne et al. (1984)) combined with a type of MHS leads to a more detailed classification. Thirty FMSs in Europe, Japan and the U.S. are classified according to this classification (Stecke and Browne, 1985).

Jaikumar (1986) gives a comparison of the use of FMSs in Japan (60 systems) and the U.S. (35 systems). He concludes that, although the systems are similar, the FMSs in Japan are better used in terms of flexibility. The number of different parts produced on Japanese FMSs was nearly ten times larger than in the U.S., and for every new part that was introduced on an American FMS, 22 parts were introduced in Japan. Development times for U.S. systems took twice as long with four times the investment in man-hours to get the system running. Ettlie (1988), however, found out that process and product flexibilities in American FMSs were well above Jaikumar's (1986) observations. Jaikumar and Van Wassenhove (1989) present results on 20 highly successful FMSs. They consider a system successful if its metal cutting time is greater than $75 \%$ of the total time, if it produces more than 100 different parts a year and if more than 25 new parts are introduced per year. For such systems, they observe average system losses of only $17 \%$ of total operating time. They observe a trend from tightly coupled cellular systems with low storage and a high degree of interdependence between machines towards integrated, independent cells distinguished by high storage or low interdependence between the machines (or both). The number of a third category of FMSs, the transfer lines, which are like tightly coupled cellular systems combined with a fixed routing, remains stable. 
Darrow (1987) has performed a survey on 253 manufacturing facilities. He mentions that the number of systems in Europe is large, but if it comes to the number of machines involved Japan has a strong lead. Darrow (1987) also comments on the product mix. In general, FMSs produce prismatic (and to a lesser extend rotational) parts including machine tools, miscellaneous industrial, aerospace and automotive products.

Ranta and Tchijov (1990) study a large FMS-database, containing data of 800 FMSs. They partition FMSs according to size and investment costs into three groups, i.e. compact, midrange and high-capacity systems. Ranta and Tchijov (1990) estimate the number of FMSs with at least two CNC machines, automated MHS and central control at 1200 FMSs in 1989 (compared to a total of 80 systems in 1980) of which $80 \%$ is compact. The estimated annual growth rate of the number of FMSs amounts to $15 \%$ until the year 2000 (Ranta and Tchijov, 1990).

\subsubsection{Must FMS be justified by faith alone?}

Kaplan (1986) clearly gives a negative answer to this question. He argues that traditional models are appropriate for the evaluation of investment proposals concerning computer integrated manufacturing. Managers need ways to apply for example a discounted cash flow approach to evaluate this new type of investment and not just rely on strategic faith. Discounted cash flow methods go wrong when rates for evaluating proposals are set too high. Kaplan (1986) also notices a bias towards incremental rather than revolutionary investment proposals. Primrose and Leonard (1991) argue that in the early years of FMS investment was done as an act of faith, where today all potential costs and benefits can be included in a financial evaluation.

Ranta and Tchijov (1990) give estimates on the investment costs involved in FMSs. The share of the cost spent on CNC-machines, MHS, system software and planning and training for the three classes of FMSs described above is given in Table 1.1. The investments in FMS appear to be large (around U.S. \$ 1 million per CNC machine). Operational costs are mainly related to labour, maintenance and tooling.

The benefits of FMSs can be splitted in benefits that are easy to quantify (tangible benefits) and those which are difficult to measure (intangible benefits) (Kaplan, 1986).

Financial analysis that focuses too much on savings that are easy to quantify, such as savings in labour cost, material or energy misses other benefits. FMSs offer opportunities for large reductions in inventory. Its 
Table 1.1 Characteristics of three classes of FMSs (source: Ranta and Tchijov (1990))

\begin{tabular}{|c|c|c|c|c|c|c|}
\hline \multirow[b]{2}{*}{ System class } & \multirow[b]{2}{*}{$\begin{array}{c}\text { Total costs } \\
\text { (in million } \\
\text { U.S. \$) }\end{array}$} & \multicolumn{4}{|c|}{ Share of costs (in \%) } & \multirow[b]{2}{*}{$\begin{array}{c}\text { Number of } \\
\text { CNC } \\
\text { machines }\end{array}$} \\
\hline & & $\begin{array}{c}\mathrm{CNC} \\
\text { machines }\end{array}$ & $\begin{array}{c}\text { Material } \\
\text { Handling } \\
\text { System }\end{array}$ & $\begin{array}{c}\text { System } \\
\text { software }\end{array}$ & $\begin{array}{c}\text { Planning } \\
\& \\
\text { Training }\end{array}$ & \\
\hline Compact & 3 & $50-55$ & $15-20$ & $20-25$ & 10 & $2-4$ \\
\hline Midrange & $4-9$ & $40-50$ & 15 & 25 & 15 & $5-15$ \\
\hline High-capacity & $10-15$ & $35-40$ & 15 & $25-30$ & $15-20$ & $15-20$ \\
\hline
\end{tabular}

flexibility gives an FMS the opportunity to react quickly to changes in demand, which also may lead to inventory reductions. Work-in-process is reduced by a vast amount. Other tangible benefits include reduced waste, scrap and rework and savings in the amount of necessary floor space. Improved quality and the possibility to easily follow customer specifications are other tangible benefits. Improved quality also gives reductions in warranty expense and gives the company a marketing advantage (Kaplan, 1986).

It is difficult to quantify all aspects of increased flexibility (see Section 1.2.2). Greater flexibility of an FMS can extend the life-time of the investment. Product life-cycles are getting shorter and FMSs can be used for several generations of products. However, quantifying these opportunities is difficult. Another intangible benefit is the reduction in throughput time and lead time. These benefits are partly incorporated in reduced inventory but other benefits such as the marketing advantages that would emerge are hard to estimate.

Investments in computer integrated manufacturing also have important learning characteristics. Kaplan (1986) compares these with buying options, where relatively small initial outlays may yield huge benefits. He concludes that there is no reason to value intangible benefits at zero. Managers should ask themselves how large benefits should be in order to justify an investment, instead of first quantifying the benefits.

Flexible manufacturing has proven to be very profitable and its concepts and technology are becoming established, which helps managers to overcome the perceived risks of this type of investment. Therefore investment in FMSs should be evaluated the same way as other investments concerning automated manufacturing are evaluated (Primrose and Leonard, 
1991). Jaikumar (1986) studied 22 systems in Japan, which all met their companies return-on-investment criterion of a three year pay back. Goldhar and Jelinek (1985) and Meredith (1987) discuss benefits in several metal working companies which adopted FMS-technology. Ranta and Tchijov (1990) conclude that small scale, compact systems and high-capacity, large scale systems (see Table 1.1) are beneficial with pay-back times of $1.5-3$ (resp. 1 - 4) years, while the returns on midrange systems are disappointing (pay-back time 5 years or more).

\subsection{Hierarchical approaches to FMS production planning}

The previous section pointed out that the benefits of FMSs are potentially high. However, these benefits can only be obtained if the FMS is properly implemented and managed. A successful implementation of an FMS corresponds to the efficient solving of the many technical, organizational and planning problems that arise when a manufacturer wants to introduce flexible manufacturing technology. Hartley (1984) gives a description of technical problems that have to be overcome like design, maintenance and control of fixtures and pallets, tool management and tool condition monitoring. These technical problems have to be solved first, but the potential benefits of an FMS can only be reached if the system is planned and controlled efficiently. In this section we review some of the literature concerning planning and control of FMSs. First, we review a number of general frameworks for planning FMSs and then concentrate on subproblems.

Several authors have presented methodologies and classifications of FMS design, planning, scheduling and control problems (Suri and Whitney, 1984; Kusiak, 1985a; Stecke, 1985; Suri, 1985; Buzacott and Yao, 1986; Kusiak, 1986; Van Looveren, Gelders and Van Wassenhove, 1986; Singhal, Fine, Meredith and Suri, 1987; Stecke, 1988), which are sometimes complementary. Most surveys describe some kind of hierarchical decision structure, relating to a variety of decisions that have to be taken concerning long-term, medium-term or short-term decisions. The differences between the different methodologies concern the number of levels or the interpretation of a specific level. Some authors give more detailed classifications of a specific level (e.g. Stecke(1983)). We now discuss some general classifications.

In our discussion we basically use the framework of Van Looveren et al. (1986). They rely on the classical three level view of the organization (Holstein, 1968) to identify subproblems, and thus establish three levels of decision making, namely the strategic, tactical and operational levels. The 
strategic level relates to long-term decisions taken by the top management, which influence the basic flexibility of the FMS. Problems involved concern the design and selection of the equipment and of the products that have to be manufactured. On the tactical level, the medium-term planning problems are addressed. Decisions taken at this level concern the off-line planning of the production system. Van Looveren et al. (1986) distinguish on this level between the batching problem and the loading problem. The batching problem is concerned with the splitting of the production orders into batches such that orders are performed on time given the limited available resources. The loading problem takes care of the actual setup of the system given the batches that are formed. Planning on the operational level is concerned with the detailed decision making required for the realtime operation of the system. A release strategy has to be developed, in which one decides which parts are fed into the system (release problem). Next the dispatching problem has to be solved to decide on the actual use of the production resources like machines, buffers and the MHS.

Buzacott and Yao (1986) give a classification of analytical models that can be used for establishing basic design concepts, detailed design, scheduling and control. Suri and Whitney (1984) describe in detail how to integrate the FMS software and hardware in the organizational hierarchy. They emphasize the value of the decision support systems as an integral part of the FMS.

Stecke (1985) distinguishes four types of problems: design, planning, scheduling and control. This description closely fits to the decision structure of Van Looveren et al. (1986). Stecke (see Stecke and Solberg (1981), Stecke $(1983 ; 1988)$ and Berrada and Stecke (1986)) has performed detailed studies on a number of these subproblems.

Kusiak (1986) makes a distinction between design and operational problems. The former relate to strategic decisions concerning the economic justification of the system and the design and selection of parts and equipment. The term operational refers to problems on the tactical and operational levels, as defined by Van Looveren et al. (1986). Kusiak (1986) splits the operational problems into four sublevels, that consider aggregate planning, resource grouping, disaggregate planning (batching and loading) and scheduling of equipments.

Kiran and Tansel (1986) use a five level decision hierarchy linked to that of Van Looveren et al. (1986). They distinguish between design, aggregate planning, system setup, scheduling and control, where design concerns the strategic level, aggregate planning and system setup take place on the tactical level and scheduling and control are on the operational level.

Singhal, Fine, Meredith and Suri (1987) discuss the problems brought forward by Buzacott and Yao (1986) and discuss the role of MS/OR 
techniques in the design, operation and control of automated manufacturing systems.

Zijm (1988) also discusses problems related to the justification, design and operation of FMSs and gives an overview of related literature.

Jaikumar and Van Wassenhove (1989) give a different outlook on FMS problems. They also present a three level model for strategic, tactical and operational planning. But, instead of stressing the complexity of FMS problems, they emphasize the use of simple models. They argue that scheduling theory and algorithms are quite sufficient for the task.

Several other authors have used the hierarchy presented by Van Looveren et al. (1986) (see Aanen (1988), Van Vliet and Van Wassenhove (1989) and Zeestraten (1989)).

A large number of mathematical and methodological tools have been used to describe and solve FMS problems on the strategic, tactical and operational level. The basic tools and techniques are (see e.g. Kusiak(1986)):

(1) Mathematical Programming;

(2) Simulation;

(3) Queuing networks;

(4) Markov processes;

(5) Petri Nets;

(6) Artificial intelligence;

(7) Perturbation analysis.

In this thesis we focus on mathematical programming techniques used for problems on the tactical and operational level of FMSs. For references concerning the other approaches we refer to the different surveys already mentioned (Stecke, 1985; Suri, 1985; Buzacott and Yao, 1986; Kusiak, 1986; Van Looveren et al., 1986).

The remainder of this section contains a discussion of FMSs problems in the framework of the three level decision hierarchy proposed by Van Looveren et al. (1986). The emphasis is on mathematical programming models for the planning and scheduling problems on the tactical and operational level.

\subsubsection{Strategic planning}

Strategic planning of flexible manufacturing activities is a responsibility for upper management. These decisions have far reaching consequences for the actual layout of the FMS and the parts that can be produced. At this level, issues relating to the design of the system must be solved. The economic justification of the system must be investigated. Van Looveren et al. (1986) 
distinguish five levels of strategic decision making:

(1) Selection of parts spectrum;

(2) Machine requirements specification;

(3) Technical alternatives;

(4) Screening of alternatives;

(5) Selection of optimal system.

The first three levels are mainly concerned with specifying the technical environment. Families of parts are selected, based on a long term production plan in which the versatility of the system is an important feature. Appropriate machines are selected to manufacture these parts. Group Technology (Hyer and Wemmerlöv, 1984) and CAD/CAM (see Section 1.2) are important decision aids in specifying several alternatives.

In the screening process the technical alternatives are evaluated using economic performance criteria. Well-known methods for screening involve queueing networks, mean value analysis, perturbation analysis and simulation. A few good alternatives are studied in detail. Finally, a selection procedure is used to select one of the remaining alternatives. Financial and technical criteria are used to select a final design, taking into account some of the potential benefits of FMSs described in Section 1.2.3. The selection step has to answer questions concerning the machines, tools, buffers and MHS. Simulation and mathematical programming (see Graves and Lamar (1983), Cooper and Jaikumar (1984), Whitney and Suri (1985), Monahan and Smunt (1987) and Avonts and Van Wassenhove (1988)) are used to help solve selection problems.

Kusiak (1986) gives an alternative classification of FMS design problems. He distinguishes economic justification, selection of parts and the selection of machine tools, storage system, MHS, fixtures and pallets, software and hardware and layout as separate, though mutually dependent, subproblems. Solot and Van Vliet (1990) give a survey of analytical models for FMS design. For other references on design and justification issues we refer to Van Looveren et al. (1986), Suri (1985) and Zijm (1988).

\subsubsection{Tactical planning}

A lot of efforts have been devoted to tactical planning problems for FMSs. In this section we review several classifications of tactical planning models, mathematical programming models and solution approaches. Special attention is given to the treatment of tooling restrictions, because these problems are the main focus of Chapters $3-6$ of this thesis.

Van Looveren et al. (1986) split tactical planning into a batching problem 
and a loading problem. The batching problem concerns the partitioning of the parts that must be produced into batches, taking into account the due dates of the parts and the availability of fixtures and pallets. The production resources are also split into a number of batches. Given these batches, the loading problem is solved, i.e. one decides in more detail how the batches are to be manufactured. Machines and tools may be pooled in groups that perform the same operations, parts are assigned to machine groups and the available fixtures and pallets are assigned to parts.

Stecke (1983) refers to tactical planning as the system setup problem. She considers five subproblems:

(1) Part type selection problem;

(2) Machine grouping problem;

(3) Production ratio (part mix) problem;

(4) Resource allocation problem;

(5) Loading problem.

In the part type selection problem a subset of parts is determined for immediate production. Grouping of the machines into groups of identical machines is pursued to increase system performance (see Stecke and Solberg (1981) and Berrada and Stecke (1986)). The production ratio problem decides on the ratios in which the parts that are selected are produced. Allocation of pallets and fixtures takes place in the resource allocation problem. The loading problem concerns the allocation of operations (that have to be performed on selected parts) and tools among the machines, subject to technological constraints such as the capacity of the tool magazine. A lot of attention has been devoted to the solution of these subproblems; we now review some important contributions in this area.

In Stecke (1983) nonlinear 0-1 mixed-integer models are proposed for the grouping and the loading problems. Linearization techniques are used for solving these problems. Berrada and Stecke (1986) develop a branch-andbound procedure for solving the loading problem.

Whitney and Gaul (1985) propose a sequential decision procedure for solving the batching (part type selection) problem. They sequentially assign part types to batches according to a probabilistic function, which is dependent on the due date of the part, the tool requirements of the part and an index describing whether a part is easy to balance with parts already selected.

Chakravarty and Shtub (1984) give several mixed-integer programming models for batching and loading problems. Kusiak (1985c) also uses group technology approaches for grouping parts into families (see also Kumar, Kusiak and Vanelli (1986)). 
Ammons, Lofgren and McGinnis (1985) present a mixed-integer formulation for a large machine loading problem and propose three heuristics for solving the problem.

Rajagopalan $(1985 ;$ 1986) proposes mixed-integer programming formulations for the part type selection, production ratio and loading problems. The first formulation is used to obtain an optimal part-mix for one planning period. A second formulation is presented to get a production plan for the entire period, which is optimal with respect to the total completion time (including processing and setup time). Two types of sequential heuristics are presented to solve the formulations. Part type priorities are determined by considering either the number of tool slots required or the processing times on the different machines.

Hwang (1986) formulates a $0-1$ integer programming model for the part type selection problem. A batch is formed by maximizing the number of parts that can be processed given the aggregate tool magazine capacity of the machines. In Hwang and Shogan (1989) this study is extended and lagrangian relaxation approaches are compared to solve the problem.

Kiran and Tansel (1986) give an integer programming formulation for the system setup problem. They consider the part type selection, production ratio, resource allocation and loading problems. The objective is to maximize the number of part types produced during the following planning period. All parts of one part type must be processed in one planning period. Kiran and Tansel (1986) propose to solve the integer programming formulation using decomposition techniques.

Stecke and Kim (1988) study the part type selection and production ratio problem. They propose a so-called flexible approach. Instead of forming batches, parts 'flow gradually' in the system. Tools can be replaced during production and not only at the start of a planning period. This offers the possibility to replace tools on some machines while production continues on the other machines. The objective is to balance the workloads of the machines. As soon as the production requirements of a part type are reached the model is solved again to determine new production ratios. Simulations are performed to compare the flexible and various batching approaches (Rajagopalan, 1985; Whitney and Gaul, 1985; Hwang, 1986). System utilization appears to be higher for the flexible approach for the types of FMSs considered.

Jaikumar and Van Wassenhove (1989) propose a three level model. On the first level the parts selected for production on the FMS and production requirements are set. A mixed-integer program is proposed that is solved by rounding off the solution values of the linear relaxation. The part type selection and loading problems are solved on the second level. The objective is to maximize machine utilization. The scheduling problem is 
solved at the third level (see Section 1.3.3). Feedback mechanisms provide feasibility of the solutions on all levels.

Kim and Yano (1992) also describe an iterative approach that solves the part type selection, machine grouping and loading problems.

The most discussed planning problems are the part type selection problem (often solved simultaneously with the production ratio problem) and the loading problem. In the last years, in particular growing attention has been paid to the loading problem and especially to the loading of tools. Therefore, we review some work that has been done in this area.

The importance of tool management is stressed by several authors (ElMaraghy, 1985; Gray, Seidmann and Stecke, 1988; Kiran and Krason, 1988; Gruver and Senninger, 1990). These authors also give an extensive review of the problems involved. Chung (1991) discusses the rough-cut tool planning problem and the tool requirements planning problem. Graver and McGinnis (1989), Daskin, Jones and Lowe (1990) and Jain, Kasilingam and Bhole (1991) discuss the tool provisioning problem, in which is decided on the number and type of tools that must be available in the system. This type of problems can also be seen as strategic problems.

Most attention has been focused on the actual tool loading problem. Most of the loading models described above include the tool loading problem. Including the capacity of the tool magazines of the machines in these models prohibits the occurrence of infeasible loadings. De Werra and Widmer (1990) propose four formulations for the tool loading problem. Several authors (Hirabayashi, Suzuki and Tsuchiya, 1984; Bard, 1988; Tang and Denardo, 1988b ; Tang and Denardo, 1988a; Widmer, 1991) have investigated tool loading problems using different objective functions. Ventura, Chen and Leonard (1988) give an overview of more work related to tool loading. Much of the present thesis, in particular Chapters $3-6$, will concentrate on tool loading models; for a further discussion of the topic we refer to these chapters and the references therein.

\subsubsection{Operational planning}

Operational planning is concerned with short-term decisions and real-time scheduling of the system. Van Looveren et al. (1986) distinguish a release and a dispatching problem. The release problem decides on the release strategy that controls the flow of parts into the system. This flow is limited for instance by the availability of pallets and fixtures. The dispatching problem relates to decisions concerning the use of machines, buffers and MHS. Procedures that have to be carried out in case of machine or system 
failure are taken care of within the dispatching problem.

Stecke (1983) gives a similar division of operational problems into scheduling and control problems. Scheduling problems concern the flow of parts through the system once it has been set up (at the tactical level). Control problems are associated with monitoring the system and keeping track of production to be sure that requirements and due dates are met.

Due to the huge number of interactions and the possibility of disturbances, the operational problems are complex. Simulation is often used to determine the performance of solution procedures for the release and dispatching problem. Chang, Sullivan, Bagchi and Wilson (1985) describe the dispatching problem as a mixed-integer programming model, which is solved using heuristics (see also Greene and Sadowski (1986) and Bastos (1988)).

The dispatching problem is often solved using (simple) dispatching rules. The purpose of these rules is to generate feasible schedules, not necessarily optimal ones. A lot of attention has been paid to the evaluation of such scheduling rules (see e.g. Panwalker and Iskander (1977), Stecke and Solberg (1981), Akella, Choong and Gershwin (1984), Shanker and Tzen (1985), Zeestraten (1989) and Montazeri and Van Wassenhove (1990)). Zijm (1988) and Blazewicz, Finke, Haupt and Schmidt (1988) give an overview on new trends in scheduling, in particular as they relate to FMS scheduling.

A strong interdependence exists between tactical and operational planning. In Spieksma, Oerlemans and Vrieze (1990) we presented a model that can be used for simultaneously formulating the system setup and scheduling problems.

\subsection{Overview of the thesis}

We have seen that FMS planning problems have a complex nature. In the previous section we presented an overview of hierarchical approaches to the planning process. Using a hierarchical framework may be helpful for identifying and understanding the fundamental underlying problems. In this thesis we study a number of such subproblems, arising within the presented framework. Emphasis lies on problems on the tactical and operational level. In Chapter 2, a hierarchical procedure is presented to solve a real-world production problem in the electronics industry. In Chapters 3 - 6 two FMS tool loading problems are studied in detail. The job grouping problem is discussed in Chapters 3, 4 and 5. In Chapter 6 another loading problem is 
studied, i.e. the problem of minimizing the number of tool switches. We take now a short walk along these chapters.

In Chapter 2, a problem of optimizing the throughput rate (production rate) in the automated manufacture of printed circuit boards (PCBs) is investigated. PCBs are widely applied in consumer electronics (e.g. computers and hi-fi) and the professional industry (e.g. telecommunications). The production of PCBs heavily relies on the use of CNC machines and the technology is continuously updated (Mullins, 1990). Production preparation for the assembly of PCBs is comparable to the system setup problem in (other) FMSs, although the type of industry is quite different from the metal working industry, which is the main area of application for FMSs (see also Van Laarhoven and Zijm (1993)). We assume that the part type selection problem has been solved (only one part type will be produced), as well as the machine grouping problem (a line of machines is available). What remains to be solved is a loading problem, which consists of the assignment of parts and equipments to the machines, taking some sequencing aspects into account. We solve this production preparation problem using a hierarchical solution procedure. A number of well-known $N P$-hard problems emerge, for which mathematical models and heuristic solution methods are developed (see also Spieksma (1992)). The hierarchical approach is successfully tested on a real-world instance in which 258 components of 39 types have to be inserted in a PCB by a line of three component-placement machines.

In Chapter 3 a loading problem (see Section 1.3.2) is studied, which arises at the tactical level in batch-industries. We present a model which aims at minimizing the number of machine setups. We assume that a number of jobs must processed on a machine. Each job requires a set of tools, which have to be present in the limited capacity tool magazine of the machine when the job is executed. We say that a group (batch) of jobs is feasible if, together, these jobs do not require more tools than can be placed in the tool magazine of the machine. Each tool is assumed to require one slot in the tool magazine. The job grouping problem is to partition the jobs into a minimum number of feasible groups. A number of authors have suggested solution approaches for the problem (Hirabayashi et al., 1984; Whitney and Gaul, 1985; Hwang, 1986; Rajagopalan, 1986; Tang and Denardo, 1988b), but no strong lower bounds on the optimal number of groups were obtained until now. We rely on a set covering formulation of the problem (Hirabayashi et al., 1984), and we solve the linear relaxation of this formulation in order to compute tight lower bounds. Since the number of variables is potentially huge, we use a column generation approach. We 
also describe some fast and simple heuristics for the job grouping problem. The result of our computational experiments on 560 randomly generated instances is that the lower bound is extremely strong: for all instances tested, the lower bound is optimal. The overall quality of the heuristic solutions appears to be very good as well.

Chapter 4 discusses a number of extensions of the previous job grouping model. First we consider the job grouping problem for one machine, where tools have different sizes (i.e., may require several slots in the tool magazine). Then we study the problem in case several machines are needed. The lower and upper bounding procedures described in Chapter 3 are generalized so as to apply to these cases as well. We present the results of computational experiments that were performed on 580 randomly generated instances. It appears that the lower bound is very strong and that the conclusions of Chapter 3 can be largely extended to this broader class of problems.

In Chapter 5 we continue our study of the job grouping problem. Attention is focused on deriving better upper bounds for the problem. A study is performed to determine the possibilities offered by local search approaches. Local search approaches explore the neighbourhood of a current solution in a smart way in order to improve this solution. Four local search approaches, viz. a simple improvement, tabu search, simulated annealing and variable-depth approach are tested. Experiments are conducted to assess the influence of the choice of starting solutions, objective functions, neighbourhood structures and stopping criteria. Computational experiments show that a majority of instances for which other (simple) heuristic procedures (presented in Chapters 3 and 4) do not produce optimal solutions can be solved optimally using a local search approach. The choice of starting solution, objective function and neighbourhood structure seems to have far more impact on the solution quality than the local search approach itself, as long as some kind of local optimum evading strategy is used.

Chapter 6 analyzes another loading problem at the tactical level of FMS planning, i.e. the tool switching problem. A batch of jobs have to be successively processed on a single flexible machine. Each job requires a subset of tools, which have to be placed in the limited capacity tool magazine of the machine before the job can be processed. The total number of tools needed exceed the capacity of the tool magazine. Hence, it is sometimes necessary to change tools between two jobs in a sequence. The problem is then to determine a job sequence and an associated sequence of 
loadings for the tool magazine, such that the total number of tool switches is minimized. This problem becomes especially crucial when the time needed to change a tool is significant with respect to the processing times of the parts, or when many small batches of different parts must be processed in succession. These phenomena have been observed in the metal-working industry by several authors (see Chapter 6). Bard (1988) and Tang and Denardo (1988a) have specifically studied the tool switching problem described above. In this chapter the problem is revisited, both from a theoretical and from a computational viewpoint. Basic results concerning the computational complexity of the problem are established. For instance, we show that the problem is already $N P$-hard when the tool magazine capacity is 2 , and we provide a new proof of the fact that, for each fixed job sequence, an optimal sequence of tool loadings can be found in polynomial time. Links between the problem and well-known combinatorial optimization problems (traveling salesman, block minimization, interval matrix recognition, etc.) are established and several heuristics are presented which exploit these special structures. Computational results are presented to compare the behaviour of the eight heuristic procedures. Also the influence of local improvement strategies is computationally assessed. 



\section{Chapter 2}

\section{Throughput rate optimization in the automated assembly of printed circuit boards*}

\subsection{Introduction}

The electronics industry heavily relies on numerically controlled machines for the placement of electronic components on the surface of printed circuit boards (PCB). These placement (or mounting, or pick-and-place) machines automatically insert components into PCB's, in a sequence determined by the input program. The most recent among them are characterized by high levels of accuracy and speed, but their throughput rates still appear to be extremely sensitive to the quality of the instructions. On the other hand, the effective programming of the machines becomes steadily more difficult in view of the increasing sophistication of the available technology. The development of optimization procedures allowing the efficient operation of such placement machines provides therefore an exciting challenge for the operations research community, as witnessed e.g. by the recent papers of Ahmadi, Grotzinger and Johnson (1988), Ball and Magazine (1988), Leipälä and Nevalainen (1989).

We propose in this paper a hierarchical approach to the problem of optimizing the throughput rate of a line of several placement machines devoted to the assembly of a single product. As usual in the study of flexible systems, the high complexity of the problem suggests to decompose it into more manageable subproblems, and to accept the solution of each

\footnotetext{
This chapter has been published as an article in Annals of Operations Research 26, 455-480, 1990.
} 
subproblem as starting point for the next one. Of course, this methodology cannot guarantee the global optimality of the final solution, even assuming that all subproblems are solved to optimality. This is even more true in the present case, where most subproblems themselves turn out to be $N P$-hard, and hence can only be approximately solved by heuristic procedures. Nevertheless, such hierarchical approaches have previously proved to deliver good quality solutions to similarly hard problems (e.g. in VLSIdesign; see Korte (1989)). They also offer the advantage of providing precise analytical models for the various facets of the global problem (see e.g. Buzacott and Yao (1986) for a discussion of analytical models in FMS).

Our approach has been tested on some industrial problems, but more experimentation would be required in order to precisely assess the quality of its performance and its range of

applicability. In particular, as pointed out by one of the referees, the validity of some of our models is conditioned by the validity of some exogenous assumptions about the nature of instances "coming up in practice" (see for instance Section 2.4.1). Even though these assumptions were fulfilled in the industrial settings that motivated our study, they may very well fail to be satisfied in other practical situations. This would then invalidate the use of the corresponding models. But we believe that the hierarchical scheme and most of the techniques presented in this paper would nevertheless remain applicable for a wide range of problem instances.

We give now a brief outline of the paper. The next section contains a more detailed description of the technological environment, and Section 2.3 provides a precise statement of the problem, and a brief account of previous related work. Sections 2.4 and 2.5 present our approach to the solution of the throughput rate optimization problem. Section 2.4 addresses the workload balancing problem for the line of machines, and Section 2.5 deals with the optimal sequencing of operations for individual machines. Both sections present mathematical models and heuristic solution methods for the various subproblems arising in our decomposition of the global problem. Finally, we describe in Section 2.6 the results delivered by our approach on a practical problem instance.

\subsection{Technological environment}

We are concerned in this paper with the automated assembly of a number of identical PCB's. For our purpose, the assembly of a PCB consists of the insertion of electronic components of prespecified types (indexed by $1, \ldots$, 
T) into prespecified locations (indexed by $1, \ldots, N$ ) on a board. Prior to operations, the components of different types are collected on different feeders (one type per feeder). Feeders are used by the placement machines as described below. We denote by $\mathrm{N}_{\mathrm{t}}$ the number of components of type $\mathrm{t}$ $(\mathrm{t}=1, \ldots, \mathrm{T})$. So, $\mathrm{N}=\sum_{\mathrm{t}=1}^{\mathrm{T}} \mathrm{N}_{\mathrm{t}}$.

We assume that a line of $\mathrm{M}$ placement machines is devoted to the assembly of the PCB's. The machines we have in mind are of the CSM (Component Surface Mounting) family. They feature a worktable, a number $S$ of feeder slots, and three pick-and-place heads (see Figure 2.1).

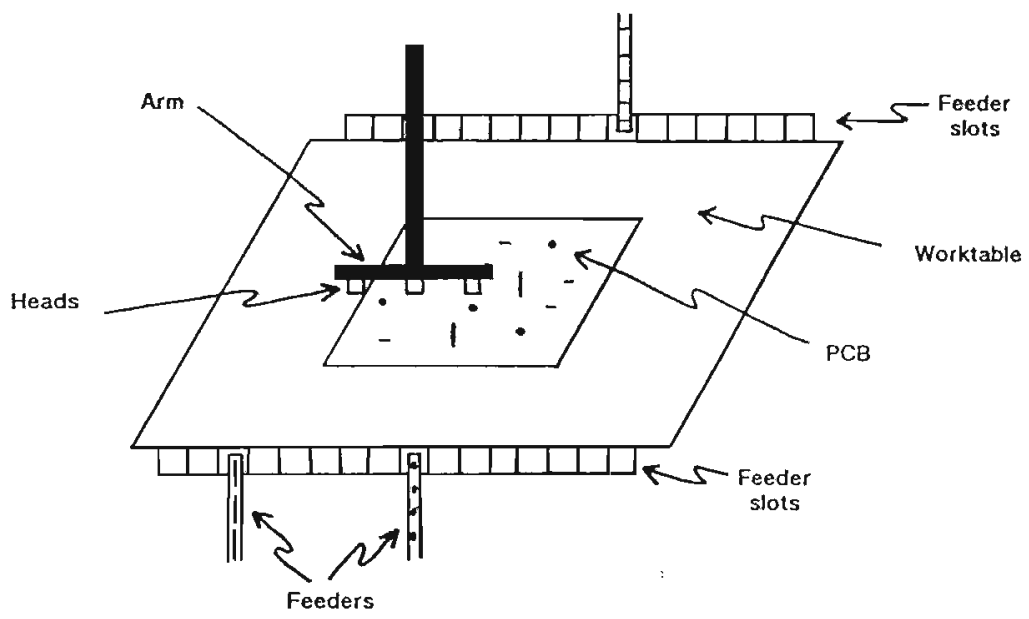

Figure 2.1 Schematic representation of a placement machine

The PCB is carried from one machine to the next by an automatic transportband, until it comes to rest on the worktable. It stays motionless during the mounting operations.

The feeder slots are fixed to two opposite sides of the worktable, $S / 2$ of them on each side. The feeders containing the components to be placed by the machine must be loaded in the slots before

the mounting begins. Depending on its type, each feeder may require 1, 2, or even more adjacent slots.

The pick-and-place heads are numbered from 1 to $3 \mathrm{M}$, with heads $3 \mathrm{~m}-2$, $3 \mathrm{~m}-1$ and $3 \mathrm{~m}$ on machine $\mathrm{m}$ (but, for short, we shall also refer to heads 1,2 and 3 of each machine). They are fixed along a same arm which remains always parallel to the side of the worktable supporting the feeder slots. The arm can move in a horizontal plane above the worktable. It can 
also perform vertical moves to allow the heads to pick components from the feeders or to insert components into the board.

Each head can carry at most one component at a time. It must be equipped with certain tools (chucks and nozzles) before it can handle any components. We call equipment the collection of tools necessary to process a given component. With every component type is associated a restricted set of alternative equipments by which it can be handled. In most situations, four or five equipments suffice to mount all component types. Changing the equipment of a head can be done either manually or automatically, depending on the technology (notice that, on certain types of machines, an equipment change can be performed automatically for heads 1 and 2 , but only manually for head 3 ). In either case, an equipment change is a time-consuming operation.

Consider now a typical pick-and-place operation, during which the machine must place components of types $\mathrm{i}, \mathrm{j}$ and $\mathrm{k}$ using heads 1,2 and 3 , respectively. Suppose for instance that these components are to be placed in the order j, i, k. Such an operation can be decomposed as follows. First, the arm moves until head 1 is positioned above a feeder of type i. Head 1 picks then a component $i$. Two more moves of the arm between the feeder slots allow heads 2 and 3 to pick components $j$ and $k$. Next, the arm brings head 2 above the location where component $\mathrm{j}$ is to be placed, and the insertion is performed. The same operation is subsequently repeated for $\mathrm{i}$ and finally for $k$.

Some remarks are in order concerning such a pick-and-place round. Notice that the picking of the components must always be performed by head 1 first, then by head 2, then by head 3 (of course, we may decide in some rounds to use only one or two heads), whereas an arbitrary sequence may be selected for their placement. Once a head has been positioned by the arm above the required feeder slot or location, the time needed to pick or to place the corresponding component depends on the type of the component, but is otherwise constant. Thus, on one machine, the only opportunities for a reduction of the total pick-and-place time reside in a clever sequencing of the operations and assignment of the feeders to feeder slots.

We have intentionally omitted many details in this brief description of the placement machines and of their functioning. For example, the insertion heads have to rotate to a predetermined angle before picking or placing components; some feeder slots or board locations are unreachable for certain heads; heads may be unavailable (e.g. broken) or may be assigned fixed equipments; the arm can only move in a limited number of directions; etc.

Some of these features (unreachable locations, unavailable heads, etc.) can 
be easily introduced in our models by setting variables to fixed values, thus resulting in a simplification of these models. Others will be implicitly incorporated in the models. For instance, parameters of the models like the pick-and-place time, or the travel time between board locations will be assumed to take into account the rotation of the heads and the restricted moves of the arm. Of course, there remains a possibility that these characteristics could be exploited explicitly to improve the performance of the machines, but we did not attempt to do so.

\subsection{The throughput rate optimization problem}

With this description of the technological constraints, we can now state a global throughput rate optimization problem as follows. Given the specifications of a PCB and of M placement machines, determine:

(1) an assignment of the components to the $M$ machines,

(2) for each machine, an assignment of feeders to feeder slots,

(3) for each machine, a sequence of pick-and-place rounds, each round consisting itself of a sequence of at most three component locations among those assigned to the machine in step (1),

(4) for each machine and for each pick-and-place round, an assignment of equipment to heads.

These decisions are to be made so that the PCB can be mounted using all $\mathrm{M}$ machines, and so as to minimize the processing time on the bottleneck machine (i.e., the machine with the longest processing time).

In our solution of this problem, we shall also take into account a secondary criterion, dictated by cost considerations. Because feeders are rather expensive, it appears desirable (at least, in the practical situations that we encountered) to minimize the total number of feeders used. Ideally, thus, all components of a same type should be processed by one machine. We shall show in Section 2.4.2 how this criterion can be accommodated.

This formulation of the throughput rate optimization problem is patterned after a (confidential) report of the Philips Center for Quantitative Methods (CQM (1988); see also van Laarhoven and Zijm (1993)). This report proposes a hierarchical decomposition of the problem, and heuristics for the resulting subproblems. Our decomposition, as well as all heuristics presented in the next two sections, are different from CQM's. Our heuristics, in particular, rely more explicitly on the precise mathematical modelling of the subproblems.

The throughput rate optimization problem is also mentioned by Ball and Magazine (1988), under somewhat simpler technological conditions. In 
particular, each machine has but one pick-and-place head. The authors investigate only in detail the sequencing of pick-and-place operations over one machine (i.e., our question (3) above).

Leipälä and Nevalainen (1989) discuss our questions (2) and (3), for a different type of one-head machines.

Ahmadi et al. (1988) consider the case of one machine featuring two heads. They address subproblems (2), (3) and (4), but their technological constraints are very different from ours, and their models do not seem to be directly applicable in our framework.

We describe in the next two sections our approach to the throughput rate optimization problem. This approach is based on a decomposition of the global problem into the following list of subproblems (which thus refines the original formulation (1) - (4) given before):

(A) determine how many components each machine must mount, and with what equipments;

(B) assign feeder types to machines;

(C) determine what components each head must mount;

(D) cluster the locations into subsets of size at most three, to be processed in one pick-and-place round;

(E) determine the sequence of pick-and-place operations to be performed by each machine;

(F) assign the feeders to feeder slots.

Subproblems (A) and (B) in this list answer together question (1) and part of question (4) above. Our main concern in solving these two subproblems will be to achieve an approximate balance of the workload over the line of machines. This will be done in Section 2.4.

Subproblems (C), (D), (E), (F) address the scheduling of individual machines, and are dealt with in Section 2.5.

In our computer experiments, the sequence of subproblems $(A)-(F)$ is solved hierarchically in a single pass (except for (E) and (F); see Section 2.5). It may be possible to use an iterative solution procedure, and to exploit the solution of certain subproblems in order to revise previous ones. We have not further explored these possibilities. 


\subsection{Workload balancing}

\subsubsection{Subproblem (A)}

\section{The model}

We proceed in this phase to a preliminary distribution of the workload over the machine line, based on the number of equipment changes for each head, and on a rough estimate of the time needed to mount each component. The latter estimate is computed as follows.

In Section 2.1, we have seen that the time needed to mount a component of type $t(t=1, \ldots, T)$ consists of two terms: a variable term measuring the travel time of the head, and a constant term $p_{t}$ representing the total time spent to pick the component when the head is directly above feeder $t$, plus the time to place the component when the head is above the desired location.

Let $v_{t}$ be an estimate of the first variable term; then, $v_{t}+p_{t}$ is an estimate of the mounting time required by each component of type $t$. Notice that, in practice, a reasonable value for $v_{t}$ does not appear too difficult to come by, e.g. by evaluating the average time required for the arm to travel from feeder slots to mounting locations. The solution of the model given below does not appear to be very sensitive to the exact value of $v_{t}$. (In our computer experimentations, we used a constant value $v$ for all $v_{t}, t=1$, ..., T.) Otherwise, solving the model for a few alternative values of $v_{t}(t=$ $1, \ldots, \mathrm{T})$ provides different initial solutions for the subsequent phases of the procedure. If necessary, after all subproblems $(A)-(F)$ have been solved, a solution to the global problem can be used to adjust the values $v_{t}$ and reiterate the whole solution procedure.

Define now two component types to be equivalent if the quantity $v_{t}+p_{t}$ is the same for both types, and if both types can be handled by precisely the same equipments. This relation induces a partition of the set of components into $\mathrm{C}$ classes, with each class containing components of equivalent types.

We are now almost ready to describe our model. We introduce first a few more parameters:

$\mathrm{Q}=$ number of available equipments; for $c=1, \ldots, C$,

$\mathrm{B}_{\mathrm{c}} \quad=$ number of components in class $\mathrm{c}$;

$\mathrm{w}_{\mathrm{c}}=$ common value of $\mathrm{v}_{\mathrm{t}}+\mathrm{p}_{\mathrm{t}}$ for the types represented in class $\mathrm{c}$;

$\mathrm{Q}(\mathrm{c})=$ set of equipments which can handle the components in class $\mathrm{c}$; 
for $\mathrm{h}=1, \ldots, 3 \mathrm{M}$,

$\mathrm{E}_{\mathrm{h}} \quad=$ time required by an equipment change for head $\mathrm{h}$.

The decision variables are:

for $\mathrm{c}=1, \ldots, \mathrm{C}$, for $\mathrm{m}=1, \ldots, \mathrm{M}$, for $\mathrm{h}=1, \ldots, 3 \mathrm{M}$, for $\mathrm{q}=1, \ldots, \mathrm{Q}$ :

$\mathrm{x}_{\mathrm{cm}}=$ number of components of class $\mathrm{c}$ to be mounted by machine $\mathrm{m}$;

$\mathrm{z}_{\mathrm{mq}}=1$ if machine $\mathrm{m}$ uses equipment $\mathrm{q}$;

$=0$ otherwise;

$\mathrm{r}_{\mathrm{h}} \quad=$ number of equipment changes required for head $\mathrm{h}$;

$\mathrm{W}=$ estimated workload of the bottleneck machine.

The optimization model for subproblem $(\mathrm{A})$ is:

$\left(M_{A}\right)$ minimize $\quad \mathrm{W}$

$$
\begin{aligned}
& \text { subject to } \sum_{m=1}^{M} x_{c m}=B_{c} \quad c=1, \ldots, C \\
& \mathrm{x}_{\mathrm{cm}} \leq \mathrm{B}_{\mathrm{c}} \sum_{\mathrm{q} \in \mathrm{Q}(\mathrm{c})} \mathrm{z}_{\mathrm{mq}} \quad \begin{array}{l}
\mathrm{c}=1, \ldots, \mathrm{C} ; \\
\mathrm{m}=1, \ldots, \mathrm{M}
\end{array} \\
& \sum_{\mathrm{q}=1}^{\mathrm{Q}} \mathrm{z}_{\mathrm{mq}} \leq \sum_{\mathrm{h}=3 \mathrm{~m}-2}^{3 \mathrm{~m}} \mathrm{r}_{\mathrm{h}}+3 \quad \mathrm{~m}=1, \ldots, \mathrm{M} \\
& \mathrm{W} \geq \sum_{c=1}^{C} w_{c} x_{c m}+\sum_{h=3 m-2}^{3 m} E_{h} r_{h} \quad m=1, \ldots, M \\
& x_{\mathrm{cm}} \geq 0 \text { integer } \quad \mathrm{c}=1, \ldots, \mathrm{C} \text {; } \\
& \mathrm{m}=1, \ldots, \mathrm{M} \\
& \mathrm{z}_{\mathrm{mq}} \in\{0,1\} \quad \mathrm{m}=1, \ldots, \mathrm{M} \\
& \mathrm{q}=1, \ldots, \mathrm{Q} \\
& r_{h} \geq 0 \text { integer } \quad h=1, \ldots, 3 M
\end{aligned}
$$


Constraints (2.1) express that all components must be mounted. Constraints (2.2) ensure that machine $\mathrm{m}$ is assigned at least one of the equipments in $Q(c)$ when $x_{c m}$ is nonzero. Constraints (2.3) together with (2.4), (2.7) and the minimization objective, impose that the number of equipment changes on each machine be equal to the number of equipments used minus three, or to zero if the latter quantity becomes negative. The right-hand side of (2.4) evaluates the processing time on machine $m$ (we assume here that the time needed to bring a new PCB on the worktable, after completion of the previous one, is always larger than the time required for an equipment change). Thus, at the optimum of $\left(M_{A}\right), W$ is equal to the maximum of these processing times.

A couple of comments are in order concerning this model. First, we could have formulated a similar model using variables $\mathrm{x}_{\mathrm{km}}$ instead of $\mathrm{x}_{\mathrm{cm}}$, with the index $\mathrm{k}$ running over all component locations, from 1 to $\mathrm{N}$. The advantage of aggregating the components into classes is that the number of variables is greatly reduced, and that some flexibility remains for the exact assignment of operations to heads. This flexibility will be exploited in the solution of further subproblems. Next, observe that we do not impose any constraint on the number of feeder slots required by a solution of $\left(M_{A}\right)$. This could, in principle, be done easily, e.g., as in the partitioning model of Ahmadi et al. (1988), but requires the introduction of a large number of new variables, resulting again from the disaggregation of classes into types. From a practical point of view, since we always allocate at most one feeder of each type per machine (remember the secondary criterion expressed in Section 2.3), the number of feeder slots never appears to be a restrictive factor, hence the solutions of $\left(M_{A}\right)$ are implementable.

In practice, the number of equipments needed to mount all components is often smaller than the number of heads available. When this is the case, we can in general safely assume that no change of equipments will be performed in the optimal solution of $\left(M_{A}\right)$ (since $\mathrm{E}_{\mathrm{h}}$ is very large). We may then replace $\left(M_{A}\right)$ by a more restrictive model, obtained by fixing $\mathrm{r}_{\mathrm{h}}=0$ for $\mathrm{h}=1, \ldots, 3 \mathrm{M}$.

\section{Complexity and solution of model $\left(M_{A}\right)$}

Every instance of the well-known set-covering problem can be polynomially transformed to an instance of $\left(M_{A}\right)$ with $\mathrm{M}=1$, which implies that model $\left(M_{A}\right)$ is already $N P$-hard when only one machine is available (we assume familiarity of the reader with the basic concepts of complexity theory; see e.g. Garey and Johnson (1979) or Nemhauser and Wolsey (1988); the proofs of all the complexity results can be found in Crama, Kolen, Oerlemans and Spieksma (1989)). 
In spite of this negative result, obtaining solutions of good quality for $\left(M_{A}\right)$ turns out to be easy in practical applications. To understand this better, notice that the number of variables in these applications is usually small. The real-world machine line which motivated our study features three machines. A typical PCB may require the insertion of a few hundred components, but these fall into five to ten classes. The number of equipments needed to mount the board (after deletion of a few clearly redundant ones) rarely seems to exceed five. So, we have to deal in $\left(M_{A}\right)$ with about 10 to 30 zero-one variables and 15 to 50 integer variables.

In view of these favourable conditions, we take a two-phase approach to the solution of $\left(M_{A}\right)$. In a first phase, we consider the relaxation of $\left(M_{A}\right)$ obtained by dropping the integrality requirement on the $x$-variables (in constraints (2.5)). The resulting mixed-integer program is easily solved by any commercial branch-and-bound code (one may also envision the development of a special code for this relaxed model, but this never appeared necessary in this context).

In the second phase we fix all $\mathrm{r}$ - and $\mathrm{z}$-variables of $\left(M_{A}\right)$ to the values obtained in the optimal solution of the first phase. We obtain in this way a model of the form:

$\left(M_{A}^{\prime}\right)$ minimize $\quad \mathrm{W}$

$$
\begin{aligned}
& \text { subject to } \sum_{m=1}^{M} x_{c m}=B_{c} \quad c=1, \ldots, C \\
& \mathrm{~W} \geq \sum_{\mathrm{c}=1}^{\mathrm{C}} \mathrm{w}_{\mathrm{c}} \mathrm{x}_{\mathrm{cm}}+\mathrm{w}_{\mathrm{m}} \quad \mathrm{m}=1, \ldots, \mathrm{M} \\
& x_{\mathrm{cm}} \geq 0 \text { integer } \quad \mathrm{c}=1, \ldots, \mathrm{C} \text {; } \\
& \mathrm{m}=1, \ldots, \mathrm{M}
\end{aligned}
$$

where some variables $x_{\mathrm{cm}}$ are possibly fixed to zero (by constraints (2.2) of $\left(M_{A}\right)$ ), and $\mathrm{W}_{\mathrm{m}}$ is the total time required for equipment changes on machine $\mathrm{m}(\mathrm{m}=1, \ldots, \mathrm{M})$.

In practice, model $\left(M_{A}\right)$ is again relatively easy to solve (even though one can show by an easy argument that $\left(M_{A}^{\prime}\right)$ is $N P$-hard). If we cannot solve it optimally, then we simply round up or down the values assumed by the $\mathrm{x}$ variables in the optimal solution of the first phase, while preserving equality in the constraints (1). In our implementation of this solution 
approach, we actually added a third phase to the procedure. The goal of this third phase is twofold: 1) improving the heuristic solutions found in the first two phases; 2) generating alternative "good" solutions of $\left(M_{A}\right)$, which can be used as initial solutions for the subsequent subproblems of our hierarchical approach.

Two types of ideas are applied in the third phase. On the one hand, we modify "locally" the solutions delivered by phase 1 or 2 , e.g. by exchanging the equipments of two machines, or by decreasing the workload of one machine at the expense of some other. On the other hand, we slightly modify model $\left(M_{A}\right)$ by imposing an upper bound on the number of components assigned to each machine, and we solve this new model.

Running the third phase results in the generation of a few alternative solutions associated with reasonable low estimates of the bottleneck workload.

\subsubsection{Subproblem (B)}

\section{The model}

At the beginning of this phase, we know how many components of each class are to be mounted on each machine, i.e. the values of the variables $\mathrm{x}_{\mathrm{cm}}$ in model $\left(M_{A}\right)$. Our goal is now to disaggregate these figures, and to determine how many components of each type must be handled by each machine. The criterion to make this decision will be the minimization of the number of feeders required (this is the secondary criterion discussed in Section 2.3).

So, consider now an arbitrary (but fixed) class $c$. Reorder the types of the components so that the types of the components contained in class $c$ are indexed by $t=1, \ldots, R$. Recall that $N_{t}$ is the total number of components of type $t$ to be placed on the board for all $t$. To simplify our notations, we also let $\mathrm{X}_{\mathrm{m}}=\mathrm{x}_{\mathrm{cm}}$ denote the number of components of class $\mathrm{c}$ to be mounted by machine $\mathrm{m}$. So, $\sum_{\mathrm{t}=1}^{\mathrm{R}} \mathrm{N}_{\mathrm{t}}=\sum_{\mathrm{m}=1}^{\mathrm{M}} \mathrm{X}_{\mathrm{m}}=\mathrm{B}_{\mathrm{c}}$. We define the following decision variables: for $t=1, \ldots, R$, for $m=1, \ldots, M$;

$$
\begin{aligned}
\mathrm{u}_{\mathrm{tm}} & =\text { number of components of type } \mathrm{t} \text { to be mounted by machine } \mathrm{m} ; \\
\mathrm{v}_{\mathrm{tm}} & =1 \text { if a feeder of type } \mathrm{t} \text { is required on machine } \mathrm{m} ; \\
& =0 \text { otherwise. }
\end{aligned}
$$

Our model for subproblem (B) is: 


$$
\begin{aligned}
& \left(M_{B}\right) \text { minimize } \sum_{\mathrm{t}=1}^{\mathrm{R}} \sum_{\mathrm{m}=1}^{\mathrm{M}} \mathrm{v}_{\mathrm{tm}} \\
& \text { subject to } \sum_{\mathrm{t}=1}^{\mathrm{R}} \mathrm{u}_{\mathrm{tm}}=\mathrm{X}_{\mathrm{m}} \quad \mathrm{m}=1, \ldots, \mathrm{M} \\
& \sum_{m=1}^{\mathrm{M}} \mathrm{u}_{\mathrm{tm}}=\mathrm{N}_{\mathrm{t}} \quad \mathrm{t}=1, \ldots, \mathrm{R} \\
& \mathrm{u}_{\mathrm{tm}} \leq \min \left(\mathrm{X}_{\mathrm{m}}, \mathrm{N}_{\mathrm{t}}\right) \mathrm{v}_{\mathrm{tm}} \quad \mathrm{t}=1, \ldots, \mathrm{R} \\
& \mathrm{m}=1, \ldots, \mathrm{M} \\
& \mathrm{u}_{\mathrm{tm}} \geq 0 \text { integer } \quad \mathrm{t}=1, \ldots, \mathrm{R} \text {; } \\
& \mathrm{m}=1, \ldots, \mathrm{M} \\
& \mathrm{v}_{\mathrm{tm}} \in\{0,1\} \quad \mathrm{t}=1, \ldots, \mathrm{R} \\
& \mathrm{m}=1, \ldots, \mathrm{M}
\end{aligned}
$$

Model $\left(M_{B}\right)$ is a so-called pure fixed-charge transportation problem (see Fisk and McKeown (1979), Nemhauser and Wolsey (1988)).

Another way of thinking about model $\left(M_{B}\right)$ is in terms of machine scheduling. Consider $\mathrm{R}$ jobs and $\mathrm{M}$ machines, where each job can be processed on any machine. Job t needs a processing time $N_{t}(t=1, \ldots, R)$ and machine $m$ is only available in the interval $\left[0, X_{m}\right](m=1, \ldots, M)$. Recall that $\sum_{t=1}^{R} N_{t}=\sum_{m=1}^{M} X_{m}$. So, if preemption is allowed, there exists a feasible schedule requiring exactly the available time of each machine. Model $\left(M_{B}\right)$ asks for such a schedule minimizing the number of preempted jobs (in this interpretation, $v_{\mathrm{tm}}=1$ if and only if job $\mathrm{t}$ is processed on machine $m$ ).

\section{Complexity and solution of model $\left(M_{B}\right)$}

The well-known partition problem can be polynomially transformed to model $\left(M_{B}\right)$, which implies that $\left(M_{B}\right)$ is $N P$-hard. Model $\left(M_{B}\right)$ can be attacked by a specialized cutting-plane algorithm for fixed-charge transportation problems (Nemhauser and Wolsey (1988)). But we choose to use instead a simple heuristic. This heuristic consists in repeatedly applying the following rule, until all component types are assigned:

Rule: assign the type (say t) with largest number $\mathrm{N}_{\mathrm{t}}$ of components to the 
machine (say $m$ ) with largest availability $X_{m}$; if $N_{t} \leq X_{m}$, delete type $t$ from the list, and reduce $X_{m}$ to $X_{m}-N_{t}$; otherwise, reduce $N_{t}$ to $N_{t}-X_{m}$, and $X_{m}$ to 0 .

Clearly, this heuristic always delivers a feasible solution of $\left(M_{B}\right)$, with value exceeding the optimum of $\left(M_{B}\right)$ by at most $M-1$ (since, of all the component types assigned to a machine, at most one is also assigned to another machine). In other words, for a class $\mathrm{c}$ containing $\mathrm{R}$ component types, the heuristic finds an assignment of types to machines requiring at most $R+M-1$ feeders. This performance is likely to be quite satisfactory, since $R$ is usually large with respect to $M$.

In situations where duplication of feeders is strictly ruled out, i.e. where all components of one type must be mounted by the same machine, we replace the heuristic rule given above by:

Modified rule: assign the type (say t) with largest number $\mathrm{N}_{\mathrm{t}}$ of components to the machine with largest availability $X_{m}$; delete type $t$ from the list; reduce $X_{m}$ to $\max \left(0, X_{m}-N_{t}\right)$.

Of course, this modified rule does not produce in general a feasible solution of $\left(M_{B}\right)$. In particular, some machine $m$ may have to mount more components of class $c$ than the amount $X_{\mathrm{m}}$ determined by subproblem (A), and the estimated workload W of the bottleneck machine may increase. In such a case, we continue with the solution delivered by the modified rule. A possible increase in estimated workload is the price to pay for imposing more stringent requirements on the solution. Before proceeding to the next phase, i.e. the scheduling of individual machines, we still have to settle one last point concerning the distribution of the workload over the machines. Namely, the solution of model $\left(M_{B}\right)$ tells us how many components of each type must be processed by each machine (namely, $\mathrm{u}_{\mathrm{tm}}$ ), but not which ones. As the latter decision does not seem to affect very much the quality of our final solution, we neglect to give here many details about its implementation. Let us simply mention that we rely on a model aiming at an even dispersion of the components over the PCB for each machine. The dispersion is measured as follows: we subdivide the PCB into cells, and we sum up the discrepancies between the expected number of components in each cell and their actual number. It is then easy to set up an integer linear programming problem, where the assignment of components to machines is modelled by $0-1$ variables, and the objective corresponds to dispersion minimization. The optimal solution of this problem determines completely the final workload distribution. 


\subsection{Scheduling of individual machines}

We concentrate in this section on one individual machine (for simplicity, we henceforth drop the machine index). Given by subproblem (B) are the locations (say $1, \ldots, N$ ) of the components to be mounted by this machine and their types $(1, \ldots, T)$. Given by subproblem (A) are the equipments $(1$, $\ldots, Q)$ to be used by the machine, and the number $r_{h}$ of equipment changes per head.

\subsubsection{Subproblem (C)}

\section{The model}

Our current goal is to determine the distribution of the workload over the three heads of the machine (a similar "partitioning" problem is treated by Ahmadi et al. (1988), under quite different technological conditions). This will be done so as to minimize the number of trips made by the heads between the feeder slots and the PCB. In other words, we want to minimize the maximum number of components mounted by a head. In general, this criterion will only determine how many components each head must pick and place, but not which ones. The latter indeterminacy will be lifted by the introduction of a secondary criterion, to be explained at the end of this subsection.

Here, we are going to use here a model very similar to $\left(M_{A}\right)$. Since we are only interested in the number of components mounted by each head, let us redefine two components as equivalent if they can be handled by the same equipments (compare with the definition used in Section 2.4.1). This relation determines $\mathrm{C}$ classes of equivalent components. As for subproblem $\left(M_{A}\right)$, we let, for $\mathrm{c}=1, \ldots, \mathrm{C}$ :

$\mathrm{B}_{\mathrm{c}} \quad=$ number of components in class $\mathrm{c}$;

$\mathrm{Q}(\mathrm{c})=$ set of equipments which can handle the components in class $\mathrm{c}$.

We use the following decision variables:

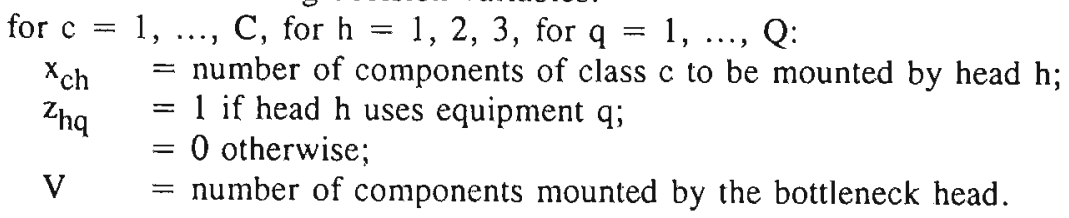

The model for subproblem (C) is: 
$\left(M_{C}\right)$ minimize $\mathrm{V}$

$$
\begin{aligned}
& \text { subject to } \sum_{\mathrm{h}=1}^{3} \mathrm{x}_{\mathrm{ch}}=\mathrm{B}_{\mathrm{c}} \quad \mathrm{c}=1, \ldots, \mathrm{C} \\
& x_{c h} \leq B_{c} \sum_{q \in Q(c)} z_{h q} \quad c=1, \ldots, C ; h=1,2,3 \\
& \sum_{\mathrm{q}=1}^{\mathrm{Q}} \mathrm{z}_{\mathrm{hq}}=\mathrm{r}_{\mathrm{h}}+1 \quad \mathrm{~h}=1,2,3 \\
& \mathrm{~V} \geq \sum_{\mathrm{c}=1}^{\mathrm{C}} \mathrm{x}_{\mathrm{cl} 1 \mathrm{i}} \quad \mathrm{h}=1,2,3 \\
& \mathrm{x}_{\mathrm{ch}} \geq 0 \text { integer } \quad \mathrm{c}=1, \ldots, \mathrm{C} ; \mathrm{h}=1,2,3 \\
& \mathrm{z}_{\mathrm{hq}} \in\{0,1\} \quad \mathrm{h}=1,2,3 ; \mathrm{q}=1, \ldots, \mathrm{Q}
\end{aligned}
$$

(recall that $r_{h}+1$ is the number of equipments allocated to head $h$ by model $\left(M_{A}\right)$ ).

\section{Complexity and solution of model $\left(M_{C}\right)$}

Again, the partition problem is easily transformed to model $\left(M_{C}\right)$, implying that the problem is $N P$-hard. Moreover, as was the case for $\left(M_{A}\right)$, model $\left(M_{C}\right)$ is actually easy to solve in practice, due to the small number of variables. Here, we can use here the same type of two-phase approach outlined for $\left(M_{A}\right)$.

As mentioned earlier, the solution of $\left(M_{C}\right)$ does not identify what components have to be mounted by each head. To answer the latter question, we considered different models taking into account the dispersion of the components over the board. However, it turned out empirically that a simple assignment procedure performed at least as well as the more sophisticated heuristics derived from these models. We describe now this procedure. Consider a coordinate axis parallel to the arm along which the three heads are mounted. We orient this axis so that the coordinates of heads 1,2 and 3 are of the form $X, X+k$ and $X+2 k$ respectively, where $k$ is the distance between two heads $(k>0)$. Notice that $X$ is variable, whereas $\mathrm{k}$ is fixed, since the arm cannot rotate. 
The idea of our procedure is to assign the component locations with smallest coordinates to head 1 , those with largest coordinates to head 3 , and the remaining ones to head 2 . Since this must be done within the restrictions imposed by $\left(M_{C}\right)$, let us consider the values $\mathrm{x}_{\mathrm{ch}}$ obtained by solving $\left(M_{C}\right)$. Then, for each $\mathrm{c}$, the components of class $\mathrm{c}$ to be mounted by head 1 are chosen to be the $x_{c 1}$ components with smallest coordinates among all components of class $c$. Similarly, head 3 is assigned the $x_{c 3}$ components with largest coordinates among the components of class $c$, and head 2 is assigned the remaining ones.

As mentioned before, this heuristic provided good empirical results. The reason for this good performance may be sought in the fact that the interhead distance $k$ is of the same order of magnitude as the length of a typical $\mathrm{PCB}$. Thus, our simple-minded procedure tends to minimize the distance travelled by the heads.

\subsubsection{Subproblem (D)}

\section{The model}

For simplicity, we first consider the case where every head has been assigned exactly one equipment (i.e., $\mathrm{r}_{1}=\mathrm{r}_{2}=\mathrm{r}_{3}=0$ in model $\left(M_{C}\right)$ ). Thus, at this point, the components have been partitioned into three groups, with group $h$ containing the $\mathrm{G}_{\mathrm{h}}$ components to be mounted by head $\mathrm{h}(\mathrm{h}=$ $1,2,3$ ). Let us further assume that $G_{1}=G_{2}=G_{3}=G$ (if this is not the case, then we add a number of "dummy" components to the smaller groups). We know that $G$ is also the minimum number of pick-and-place rounds necessary to mount all these components. We are now going to determine the composition of these rounds, with a view at minimizing the total travel time of the arm supporting the heads.

Suppose that the components in each group have been (arbitrarily) numbered $1, \ldots, \mathrm{G}$. Consider two components $\mathrm{i}$ and $\mathrm{j}$ belonging to different groups, and assume that these components are to be mounted successively, in a same round. We denote by $\mathrm{d}_{\mathrm{ij}}$ the time necessary to reposition the arm between the insertions of $i$ and $j$. For instance, if $i$ is in group $1, j$ is in group 2 , and $\mathrm{i}$ must be placed before $\mathrm{j}$, then $\mathrm{d}_{\mathrm{ij}}$ is the time required to bring head 2 above the location of $\mathrm{j}$, starting with head 1 above $\mathrm{i}$.

For a pick-and-place round involving three components $\mathrm{i}, \mathrm{j}, \mathrm{k}$, we can choose arbitrarily the order in which these components are mounted (see Section 2.2). Therefore, an underestimate for the travel time of the arm between the first and the third placements of this round is given by:

(i) $d_{i j k}=\min \left\{d_{i j}+d_{j k}, d_{i k}+d_{k j}, d_{j i}+d_{i k}\right\}$ if none of $i, j, k$ is a dummy; 
(ii) $\mathrm{d}_{\mathrm{ijk}}=\mathrm{d}_{\mathrm{ij}}$ if $\mathrm{k}$ is a dummy;

(iii) $\mathrm{d}_{\mathrm{ijk}}=0$ if at least two of $\mathrm{i}, \mathrm{j}, \mathrm{k}$ are dummies.

Let us introduce the decision variables $u_{i j k}$, for $i, j, k \in\{1, \ldots, G\}$, with the interpretation:

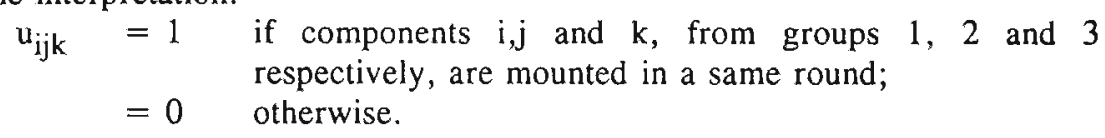

Then, our optimization model for subproblem (D) is:

$$
\begin{array}{rlr}
\left(M_{D}\right) \text { minimize } & \sum_{i=1}^{\mathrm{G}} \sum_{j=1}^{\mathrm{G}} \sum_{\mathrm{k}=1}^{\mathrm{G}} \mathrm{d}_{\mathrm{ijk}} \mathrm{u}_{\mathrm{ijk}} & \\
\text { subject to } & \sum_{\mathrm{i}=1}^{\mathrm{G}} \sum_{\mathrm{j}=1}^{\mathrm{G}} \mathrm{u}_{\mathrm{ijk}}=1 & \mathrm{k}=1, \ldots, \mathrm{G} \\
& \sum_{\mathrm{i}=1}^{\mathrm{G}} \sum_{\mathrm{k}=1}^{\mathrm{G}} \mathrm{u}_{\mathrm{ijk}}=1 & \mathrm{j}=1, \ldots, \mathrm{G} \\
& \sum_{\mathrm{j}=1}^{\mathrm{G}} \sum_{\mathrm{k}=1}^{\mathrm{G}} \mathrm{u}_{\mathrm{ijk}}=1 & \mathrm{i}=1, \ldots, \mathrm{G} \\
& \mathrm{u}_{\mathrm{ijk}} \in\{0,1\} & \mathrm{i}, \mathrm{j}, \mathrm{k}=1, \ldots, \mathrm{G} .
\end{array}
$$

An optimal solution of $\left(M_{D}\right)$ determines $G$ clusters, of three components each, such that the sum of the (underestimates of the) travel times "within clusters" is minimized.

In cases where some or all of the heads have been assigned more than one equipment in model $\left(M_{A}\right)$, we adapt our approach as follows. Let $\mathrm{q}_{\mathrm{h}}$ be the first equipment to be used by head $h$, and $G_{h}$ be the number of components which can be handled by $\mathrm{q}_{\mathrm{h}}$ among those to be mounted by head $\mathrm{h}(\mathrm{h}=1$, 2,3 ). Say for instance that $G_{1} \leq G_{2} \leq G_{3}$. We can now set up a model similar to $\left.M_{D}\right)$ for the clustering of these $G_{1}+G_{2}+G_{3}$ components. Any feasible solution of this model determines exactly $G_{1}$ clusters containing no dummy components. These clusters correspond to our first $G_{1}$, pick-and-place rounds, to be performed by equipments $q_{1}, q_{2}$ and $q_{3}$. Next, $q_{1}$ is replaced by a new equipment $q_{4}$, and the process can be 
repeated with $q_{4}, q_{2}$ and $q_{3}$.

\section{Complexity and solution of $\left(M_{D}\right)$}

Model $\left(M_{D}\right)$, with arbitrary coefficients $\mathrm{d}_{\mathrm{ijk}}$, has been studied in the literature under the name of 3-dimensional assignment problem. The problem is known to be $N P$-hard (see Garey and Johnson (1979)). But observe that, in our case, the coefficients $\mathrm{d}_{\mathrm{ijk}}$ are of the very special type defined by (i) - (iii). Moreover, the travel times $d_{i j}(i, j=1, \ldots, G)$ are themselves far from arbitrary; in particular, they satisfy the triangle inequality: $\mathrm{d}_{\mathrm{ij}} \leq \mathrm{d}_{\mathrm{ik}}+\mathrm{d}_{\mathrm{kj}}$ for $\mathrm{i}, \mathrm{j}, \mathrm{k}=1, \ldots, \mathrm{G}$. However, even under these added restrictions model $\left(M_{D}\right)$ remains $N P$-hard (Crama and Spieksma (1992)).

A number of heuristic and exact algorithms have been proposed to solve the 3-dimensional assignment problem (see e.g. Frieze and Yadegar (1981) and the references therein). In view of the role of $\left(M_{D}\right)$ as a subproblem in the hierarchy $(A)-(F)$, and of the special structure of its cost coefficients, we opt here for a specialized heuristic procedure.

Our heuristic works in two phases. We start by solving an (ordinary) assignment problem, obtained by disregarding the components of the third group. Thus, we solve:

(AP1) minimize $\sum_{i=1}^{\mathrm{G}} \sum_{j=1}^{\mathrm{G}} \mathrm{d}_{\mathrm{ij}} \mathrm{u}_{\mathrm{ij}}$

$$
\begin{aligned}
& \text { subject to } \sum_{j=1}^{\mathrm{G}} \mathrm{u}_{\mathrm{ij}}=1 \quad \mathrm{i}=1, \ldots, \mathrm{G} \\
& \sum_{i=1}^{G} u_{i j}=1 \quad j=1, \ldots, G \\
& \mathrm{u}_{\mathrm{ij}} \in\{0,1\} \quad \mathrm{i}, \mathrm{j}, \mathrm{k}=1, \ldots, \mathrm{G},
\end{aligned}
$$

where $\mathrm{d}_{\mathrm{ij}}=0$ if either $\mathrm{i}$ or $\mathrm{j}$ is dummy. An optimal solution $\mathrm{u}^{*}$ of (API) can be computed in time $0\left(\mathrm{~N}^{3}\right)$ (Papadimitriou and Steiglitz (1982). Let now $\mathrm{A}=\left\{(\mathrm{i}, \mathrm{j}): \mathrm{u}_{\mathrm{ij}}^{*}=1\right\}$. Thus, $\mathrm{A}$ is the set of pairs $(\mathrm{i}, \mathrm{j})$ matched by the solution of $(A P I)$. The second phase of our heuristic consists in assigning the (previously disregarded) components of the third group to the pairs in A. Formally, we solve: 
(AP2) minimize $\sum_{(\mathrm{i}, \mathrm{j}) \in \mathrm{A}} \sum_{\mathrm{k}=\mathrm{l}}^{\mathrm{G}} \mathrm{d}_{\mathrm{jjk}} \mathrm{u}_{\mathrm{ijk}}$

$$
\begin{aligned}
& \text { subject to } \sum_{k=1}^{G} u_{i j k}=1 \quad(i, j) \in A \\
& \sum_{(i, j) \in A} u_{i j k}=1 \quad k=1, \ldots, G \\
& \mathrm{u}_{\mathrm{ijk}} \in\{0,1\} \quad(\mathrm{i}, \mathrm{j}) \in \mathrm{A} ; \mathrm{k}=1, \ldots, \mathrm{G} .
\end{aligned}
$$

The optimal solution of $(A P 2)$ can be obtained in time $\mathrm{O}\left(\mathrm{G}^{3}\right)$ and provides a heuristic solution of $\left(M_{D}\right)$. Frieze and Yadegar (1981) proposed a closely related heuristic for general 3-dimensional assignment problems, and observed its good empirical performance.

Let $B_{3}$ denote the optimal value of $(A P 2)$. The notation $B_{3}$ is a reminder that, in the first phase of our heuristic, we arbitrarily decided to disregard the components from the third group. Of course, similar procedures could be defined, and corresponding bounds $\beta_{1}$ and $B_{2}$ would be derived, by initially disregarding the components from either group 1 or group 2 .

In our computer implementations, we compute the three bounds, $\beta_{1}, \beta_{2}$, $B_{3}$, and we retain the clustering of the components corresponding to the smallest bound. In Crama and Spieksma (1992) it is proven that this bound is never worse than $4 / 3$ time the optimal value for any instance of $\left(M_{D}\right)$. The computer experiments reported in Crama and Spieksma (1992) indicate that the practical performance of this heuristic is excellent.

\subsubsection{Subproblem (E).}

The solution of subproblem (D) has delivered a list $\mathrm{C}_{1}, \ldots, \mathrm{C}_{\mathrm{G}}$ of clusters, with each cluster containing (at most) three components to be placed in a same round (if some heads must use more than one equipment, then we successively consider several such lists, where each list consists of clusters which can be processed without equipment changes). Subproblem (E) asks for the sequence of pick-and-place operations to be performed by the machine, given this list of clusters.

This problem has been studied by Ball and Magazine (1988) and Leipälä and Nevalainen (1989), for machines featuring only one insertion head. In both papers, the authors observed that the decisions to be made in subproblem (E) are highly dependent on the assignment of feeders to feeder 
slots (i.e. on the solution of our subproblem (F)), and conversely. On the other hand, a model simultaneously taking into account both subproblems is far too complicated to present any practical value.

We choose therefore an approach already suggested by Leipälä and Nevalainen (1989). Namely, we first solve subproblem (E); using this solution as input, we compute a solution of subproblem (F), which is used in turn to revise the solution of subproblem (E), and so on. This process is iterated until some stopping condition is verified.

\section{The models}

According to the previous discussion, we need two models for subproblem (E): the first one to be used when no feeder assignment is yet known, and the second one taking into account a given feeder assignment. In either case, we reduce (E) to the solution of a shortest Hamiltonian path problem (see Lawler, Lenstra, Rinnooy Kan and Shmoys (1985)) over the set of clusters $\left\{\mathrm{C}_{1}, \ldots, \mathrm{C}_{\mathrm{G}}\right\}$ : for $\mathrm{i}, \mathrm{j}=1, \ldots, \mathrm{G}$, we define a cost (travel time) $c(i, j)$ for processing $C_{i}$ immediately before $C_{j}$; the problem is then to find a permutation $\sigma=\left(\sigma_{1}, \ldots, \sigma_{\mathrm{G}}\right)$ of $\{1, \ldots, \mathrm{G}\}$ which minimizes

$$
c(\sigma)=\sum_{i=1}^{\mathrm{G}-1} c\left(\sigma_{i}, \sigma_{i+1}\right)
$$

The definition of $c(i, j)$ depends on the given feeder assignment (if any), as explained hereunder. Consider first the situation where feeders are already assigned to feeder slots, and let $\mathrm{C}_{i}, \mathrm{C}_{\mathrm{j}}$ be two arbitrary clusters. In this case, the appropriate definition of $c(1, j)$ is given by CQM (1988) as follows. Denote by $l_{1}, l_{2}, l_{3}$ the component locations in $C_{i}$, where $l_{h}$ is to be processed by head $h(h=1,2,3)$. We assume that the feeder needed for $l_{h}$ is in slot $s_{h}(h=1,2,3)$. Similarly, $l_{4}$ is the location to be processed by head 1 in cluster $C_{j}$, and slot $s_{4}$ contains the corresponding feeder (we assume for simplicity that $C_{i}$ and $C_{j}$ consist of exactly three locations; obvious modifications of our description are required when this is not the case).

Suppose now for a moment that $l_{1}, l_{2}$ and $l_{3}$ are to be mounted in the order $\pi=\left(\pi_{1}, \pi_{2}, \pi_{3}\right)$, where $\left(\pi_{1}, \pi_{2}, \pi_{3}\right)$ is a permutation of $\{1,2,3\}$. For this fixed order, we can easily compute the time (say $c_{i j}(\pi)$ ) required to carry out the following operations: starting with head 1 above slot $s_{1}$ sequentially pick one component from each of $s_{1}, s_{2}, s_{3}$ using heads 1,2 , 3 respectively; mount $l_{\pi}, 1_{\pi_{2}}, 1_{\pi_{3}}$ in that order; bring head 1 above slot $s_{4}$. Obviously, in an optinlal pick-and-place sequence, we would select the 
permutation $\pi^{*}$ of $\{1,2,3\}$ which minimizes $c_{i j}(\pi)$. We accordingly define: $c(i, j)=c_{i j}\left(\pi^{*}\right)$.

Now, if $\sigma$ is any permutation of $\{1, \ldots, G\}$, then $c(\sigma)$ (given by (2.8)) is the time required by a complete pick-and-place sequence processing the clusters in the order $\left(\sigma_{1}, \ldots, \sigma_{G}\right)$. The shortest Hamiltonian path problem with costs $c_{i j}$ provides thus a natural model for subproblem $(E)$. As a last remark on this model, notice that the computation of $c_{i j}(\pi)$ can be simplified by dropping from its definition those elements which are independent of $\pi$ or $\sigma$. Namely, we can use a "modified $c_{i j}(\pi)$ " defined as the time needed, starting with head 3 above $s_{3}$, to bring successively head $\pi_{1}$ above $1_{\pi_{1}}$, head $\pi_{2}$ above $1_{\pi_{2}}$, head $\pi_{3}$ above $l_{\pi}$ and finally head 1 above $s_{4}$. Let us return now to the initial solution of $(E)$, when the feeder positions are still unknown. Since this initial sequence will be modified by the subsequent iterations of our procedure, it does not seem necessary at this stage to look for a solution of very high quality (actually, one may even argue that an initial sequencing of lower quality is desirable since it provides more flexibility in the next phases of the procedure; see e.g. Leipälä and Nevalainen (1989) for more comments along this line). Accordingly, we define the coefficients $c(i, j)$ for our initial traveling salesman problem as rough estimates of the actual travel times. We experimented here with a couple of possible definitions, which seem to lead to comparable results (in terms of the final solution obtained). One such definition is as follows. Let $g_{i}$ and $g_{j}$ be the centres of gravity of the clusters $C_{i}$ and $C_{j}$, respectively. Let $s$ be the feeder slot minimizing the total distance from $g_{i}$ to $s$ to $g_{j}$. Then, $c(i, j)$ is the time needed for the arm to travel this total distance.

\section{Complexity and solution of the models}

The shortest Hamiltonian path problem is closely related to the traveling salesman problem, and is well-known to be $N P$-hard, even when the costs $c(i, j)$ satisfy the triangle inequality (Lawler et al. (1985)). Many heuristics have been devised for this problem, and we have chosen to experiment with two of the simplest: nearest neighbour (with all possible starting points) and farthest insertion, which respectively run in $\mathrm{O}\left(\mathrm{G}^{3}\right)$ and $\mathrm{O}\left(\mathrm{G}^{2}\right)$ steps (we refer to Lawler et al. (1985) for details on these procedures). Both heuristics delivered results of comparable quality. 


\subsubsection{Subproblem (F)}

\section{The model}

As input to this subproblem, we are given the types $(1, \ldots, \mathrm{T})$ and the locations $(1, \ldots, N)$ of the components to be mounted, where $(1, \ldots, N)$ is the mounting sequence determined by the previous solution of subproblem (E). Our problem is now to allocate each feeder $1, \ldots, \mathrm{T}$ to one of the feeder slots $1, \ldots, S$, so as to minimize the total mounting time (for the sake of clarity, we first assume that every feeder can be loaded in exactly one slot; we indicate later how our model can be modified when some feeders require two or more slots).

We use the decision variables $v_{t s}(t=1, \ldots, T ; s=1, \ldots, S)$ with the interpretation:

$\mathrm{v}_{\mathrm{ts}}=1$ if feeder $\mathrm{t}$ is loaded in slot $\mathrm{s}$;

$=0$ otherwise.

These variables must obey the following restrictions, expressing that every feeder occupies exactly one slot, and no slot contains two feeders:

$$
\begin{array}{ll}
\sum_{\mathrm{s}=1}^{S} v_{t s}=1 & \mathrm{t}=1, \ldots, \mathrm{T} \\
\sum_{\mathrm{t}=1}^{\mathrm{T}} \mathrm{v}_{\mathrm{ts}} \leq 1 & \mathrm{~s}=1, \ldots, \mathrm{S} \\
\mathrm{v}_{\mathrm{ts}} \in\{0,1\} & \mathrm{t}=1, \ldots, \mathrm{T} ; \\
& \mathrm{s}=1, \ldots, \mathrm{S}
\end{array}
$$

Before describing the other elements of our model, we first introduce some terminological conventions. We say that a movement of the arm is a feederboard movement if it occurs between the last picking and the first placing of a same round, or between the last placing of a round and the first picking of the next one. By contrast, a feeder-feeder movement takes place between two pickings of a same round.

Consider now a fixed solution $v_{t s}(t=1, \ldots, T ; s=1, \ldots, S)$ of $(2.9)$ (2.11). For the corresponding assignment of feeders to slots, the total mounting time of the PCB can be broken up into three terms: 
1) a term $\sum_{t=1}^{T} \sum_{s=1}^{S} a_{t s} v_{t s}$, where $a_{t s}$ is the total time spent in feeder-board movements from or to feeder $t$, when feeder $t$ is loaded in slot $s$; this term represents the total feeder-board traveltime; notice that the value of each coefficient $a_{t s}$ is completely determined by the technological features of the machine, and by the sequence of pickand-place operations to be performed by the machine (i.e., by the solution of subproblem (E));

2) a term $\sum_{\mathrm{p}, \mathrm{t}=1}^{\mathrm{T}} \sum_{\mathrm{r}, \mathrm{s}=1}^{\mathrm{S}} \mathrm{b}_{\mathrm{prts}} \mathrm{v}_{\mathrm{pr}} \mathrm{v}_{\mathrm{ts}}$, where $b_{\text {prts }}$ is the total time spent in feeder-feeder movements between feeders $p$ and $t$, when feeder $\mathrm{p}$ is in slot $\mathrm{r}$ and feeder $\mathrm{t}$ is in slot $\mathrm{s}$; this term gives the total feeder-feeder travel time; here again the coefficients $b_{\text {prts }}$ are easily computed.

3) a term accounting for all other operations (picking and placing of all components, and travel time between placements of a same round); for a fixed pick-and-place sequence, this term is independent of $v_{t s}$.

According to this discussion, our model for subproblem (F) can be formulated as:

$\left(M_{F}\right)$ minimize $\sum_{\mathrm{t}=1}^{\mathrm{T}} \sum_{s=1}^{\mathrm{S}} \mathrm{a}_{\mathrm{ts}} \mathrm{v}_{\mathrm{ts}}+\sum_{\mathrm{p}, \mathrm{t}=1}^{\mathrm{T}} \sum_{\mathrm{r}, \mathrm{s}=1}^{\mathrm{S}} \mathrm{b}_{\mathrm{prts}} \mathrm{v}_{\mathrm{pr}} \mathrm{v}_{\mathrm{ts}}$ subject to $(2.9),(2.10),(2.11)$.

Problem $\left(M_{F}\right)$ is a quadratic assignment problem (see Burkard (1984)). As mentioned earlier, this formulation can be easily modified to accommodate additional restrictions. For instance, if feeder $t$ must occupy two slots, we reinterpret:

$$
\begin{aligned}
\mathrm{v}_{\mathrm{ts}} & =1 \text { if feeder } \mathrm{t} \text { is loaded in slots } \mathrm{s} \text { and } \mathrm{s}+1 ; \\
& =0 \text { otherwise. }
\end{aligned}
$$

Straightforward restrictions must then be added to (2.9) - (2.11) to preclude the assignment of any feeder to slot $s+1$ when $v_{t s}=1$. This can also be achieved while preserving the quadratic assignment structure of $\left(M_{F}\right)$, by raising all coefficients $b_{p, s}+1, t, s$ to very high values.

As a last remark on $\left(M_{F}\right)$, let us observe that this model boils down to a linear assignment problem for machines featuring only one insertion head. On the other hand, Leipälä and Nevalainen (1989) proposed a quadratic assignment formulation of the feeder assignment subproblem (F) for another type of one-head machines. This discrepancy is obviously due to the different technologies. 


\section{Complexity and solution of $\left(M_{F}\right)$}

The quadratic assignment problem is well-known to be $N P$-hard, and to be particularly difficult to solve exactly for values of $T$ and $S$ larger than 20 (Burkard (1984)). A typical instance of $\left(M_{F}\right)$ may involve as many as 20 feeder types and 60 slots, and hence must be attacked by heuristic methods. We have used for $\left(M_{F}\right)$ a local improvement method, based on pairwise exchanges of feeders (see (Burkard (1984)). This procedure starts with an initial solution of (2.9) - (2.11), and applies either of the following steps, as long as they improve the objective function value in $\left(M_{F}\right)$ :

Step 1:

Step 2:

move a feeder from its current slot to some empty slot; interchange the slot assignments of two feeders.

To determine an initial assignment of feeders to slots, we proceed in two phases. First, we solve the assignment problem $\left(M_{F}^{\prime}\right)$ obtained by setting all coefficients $b_{\text {prts }}$ to zero in $\left(M_{F}\right)$ (this amounts to disregarding the feederfeeder movements of the arm). Let $\mathrm{v}^{*}$ be an optimal solution of $\left(M_{F}^{\prime}\right)$.

Next, we consider those feeders (say $1, \ldots, P$ ) whose components are only picked by head 2 . Observe that the associated variables $v_{t s}(t=1, \ldots, P ; s$ $=1, \ldots, S)$ do not appear in the objective function of $\left(M_{F}\right)$, since there are no feeder-board movements to or from these feeders (i.e., $\mathrm{a}_{\mathrm{ts}}=0$ for $\mathrm{t}_{*}=$ $1, \ldots, P ; s=1, \ldots, S)$. Consequently, the value of these variables in $v^{*}$ is only conditioned by the constraints (2.9) - (2.11), and may as well be random. In order to determine more meaningful values for these variables, we solve the restriction of $\left(M_{F}\right)$ obtained by setting $\mathrm{v}_{\mathrm{ts}}=\mathrm{v}_{\mathrm{ts}}^{*}$ for $\mathrm{t}=\mathrm{P}+1$, $\ldots, T$ and $s=1, \ldots, S$. It is easy to see that this is again a linear assignment problem, aiming at the minimization of the total feeder-feeder travel time under the partial assignment $v_{\mathrm{ts}}^{*}(\mathrm{t}=\mathrm{P}+1, \ldots, \mathrm{T} ; \mathrm{s}=1, \ldots$, $S)$. The optimal solution of this problem together with the values $v_{t s}^{*}(t=$ $\mathrm{P}+1, \ldots, \mathrm{T} ; \mathrm{S}=1, \ldots, \mathrm{S})$, provides the initial solution for the improvement procedure described above.

\subsection{An example}

We discuss in this section the performance of our heuristics on a problem instance described in CQM (1988). The placement line under consideration consists of three machines. The third head is broken and unavailable on machine 3 . The 258 components to be mounted on the PCB are grouped in 39 types (actually, the PCB is partitioned into three identical blocks, of 86 components each; we shall make use of this peculiarity in the solution of 
subproblem (A)). Three distinct equipments suffice to handle all the component types, moreover, each type can be handled by exactly one of these three equipments.

For the sake of comparison, let us mention that CQM (1988) evaluates to 74,65 and 81 seconds respectively the mounting times required by the three machines for the actual operations sequence implemented by the plant (notice that this sequence is not known in full detail, and that these "plant times" appear to be underestimates). The hierarchical decomposition and the heuristics developed in CQM (1988) produce a solution with mounting times $68.41,66.52$ and 68.88 seconds for the three machines. A still better solution is obtained in CQM (1988) after imposing that the equipments used remain fixed as in the plant situation. Under these conditions, production times of $66.12,65.25$ and 65.47 are achieved on the three machines, i.e. an improvement of at least 18 percent of the bottleneck time with respect to the plant solution. To fully appreciate these figures, one should also know that a constant time of 106 seconds is needed for the pick-and-place operations alone, independently of the production sequence (see Section 2.2). These unavoidable 106 seconds represent more than half of the total mounting time required by the CQM solutions.

\subsubsection{Subproblem (A)}

We take up now our subproblem (A). With a constant estimate of $v=0.3$ (secs) for the travel time of the heads between two insertions, the components fall into five classes, characterized by the parameters in Table 2.1 .

Table 2.1 Parameters for subproblem (A)

\begin{tabular}{|c|c|c|c|c|c||}
\hline Class & 1 & 2 & 3 & 4 & 5 \\
\hline $\mathrm{B}_{\mathrm{c}}$ & 201 & 27 & 24 & 3 & 3 \\
$\mathrm{w}_{\mathrm{c}}$ & 0.6 & 1.35 & 0.75 & 1.65 & 1.15 \\
$\mathrm{Q}(\mathrm{c})$ & $(1)$ & $(2)$ & $(3)$ & $(3)$ & $(3)$ \\
\hline
\end{tabular}

We set up model $\left(M_{A}\right)$ with these parameters and $\mathrm{E}_{\mathrm{h}}=2(\mathrm{~h}=1, \ldots, 8)$ (and the obvious modifications implied by the unavailability of head 9). This model is easily solved by the approach described in Section 2.4.1. Notice that the relaxation of $\left(M_{A}\right)$ obtained by dropping the integrality requirement for the $\mathrm{x}$-variables has several alternative optima. As expected, $r_{h}=0(h=1, \ldots, 8)$ in all these optimal solutions, i.e. equipment 
changes are ruled out.

As explained in Section 2.4.1, the solutions found for subproblem (A) can be considered as alternative inputs for the subsequent subproblems in the decomposition. In the present case, most of these solutions led us to production plans with processing times of 66 to 68 seconds. To illustrate the next steps of our approach, we shall concentrate now on a specific solution of $\left(M_{A}\right)$, derived as follows.

We mentioned before that our PCB consists of three identical blocks. So, rather than solving $\left(M_{A}\right)$ for the complete board, we can solve first the model corresponding to one of the blocks, and eventually multiply all figures by 3 . A workload distribution obtained in that way is displayed in Table 2.2 .

Table 2.2 Workload distribution

\begin{tabular}{|c|c|c|c|}
\hline Machine & 1 & 2 & 3 \\
\hline Equipment & 1 & 1,3 & 1,2 \\
\hline$x_{c m}=$ number of & & $x_{12}=57$ & \\
components of class & $x_{11}=102$ & $\begin{array}{l}x_{32}=24 \\
x_{42}=3 \\
\text { c on machine } m\end{array}$ & $x_{13}=42$ \\
& & $x_{23}=27$ \\
\hline
\end{tabular}

\subsubsection{Subproblem (B)}

Since all components of class 2 are to be handled by machine 3 , and all components of classes $3,4,5$ by machine 2 , we see that the distribution shown in Table 2.2 need only be further refined for class 1 . Specifically, 28 components types are represented in class 1 . The number of components of each type $(1, \ldots, 28)$ is given in Table 2.3 .

Table 2.3 Number of components of each type for subproblem (B)

\begin{tabular}{||c|cccccccccccccc|}
\hline Type & 1 & 2 & 3 & 4 & 5 & 6 & 7 & 8 & 9 & 10 & 11 & 12 & 13 & 14 \\
\hline $\mathrm{N}_{\mathrm{t}}$ & 24 & 18 & 18 & 15 & 12 & 9 & 9 & 9 & 9 & 6 & 6 & 6 & 6 & 6 \\
\hline
\end{tabular}

\begin{tabular}{|c|cccccccccccccc|}
\hline \hline Type & 15 & 16 & 17 & 18 & 19 & 20 & 21 & 22 & 23 & 24 & 25 & 26 & 27 & 28 \\
\hline $\mathrm{N}_{\mathrm{t}}$ & 6 & 6 & 3 & 3 & 3 & 3 & 3 & 3 & 3 & 3 & 3 & 3 & 3 & 3 \\
\hline
\end{tabular}

The heuristic rule described in Section 2.4 .2 produces the assignment 
shown in Table 2.4. Observe that each type is assigned to exactly one machine, and hence exactly one feeder of each type will be needed in the final solution (in particular, the heuristic delivers here an optimal solution of $\left(M_{B}\right)$ ).

Table 2.4 Assignment of component types to machines

\begin{tabular}{|c|c||}
\hline \hline Machine & Types \\
\hline 1 & $1,2,3,5,10,11,14,17,20,23,26$ \\
2 & $4,6,8,12,15,18,21,24,27$ \\
3 & $7,9,13,16,19,22,25,28$ \\
\hline
\end{tabular}

\subsubsection{Subproblem (C)}

Since model $\left(M_{C}\right)$ attempts to minimize the maximum workload of the heads (per machine), we obviously find in this case an assignment of the type given in Table 2.5 .

Table 2.5 Assignment for subproblem (C)

\begin{tabular}{|lcccccccc||}
\hline \hline Head & 1 & 2 & 3 & 4 & 5 & 6 & 7 & 8 \\
\hline Equipment & 1 & 1 & 1 & 1 & 1 & 3 & 1 & 2 \\
\hline Number of components & 34 & 34 & 34 & 29 & 28 & 30 & 42 & 27 \\
\hline
\end{tabular}

The components to be mounted by heads $1,2,3,4,5$ are further identified as

explained at the end of Section 2.5.2. In the present case, this amounts to assigning to head 1 all components of block 1 , to head 2 all components of block 2 , and to head 3 all components of block 3 , among those previously assigned to machine 1 .

\subsubsection{Subproblem (D)}

We solve now the 3-dimensional assignment model $\left(M_{D}\right)$ for each of the three machines. Since machine 3 only has two heads, $\left(M_{D}\right)$ actually boils down to the assignment problem ( $A P I)$ for this machine, and hence can be solved exactly (optimal value: 3.26 secs).

For machines 1 and 2, we solve $\left(M_{D}\right)$ using the heuristics described in 
Section 2.5.2. These heuristics deliver for machine 1 a very good clustering of the components (value: 4.95 secs), where each cluster simply contains corresponding components from each block of the PCB. We obtain for machine 2 a clustering with value 8.95 secs.

\subsubsection{Subproblem (E) and (F)}

These two subproblems are solved alternately and iteratively for each machine. On machine 2, for instance, the first Hamiltonian path (corresponding to travel times between centres of gravity of the clusters) has value 13.16 secs. An initial feeder assignment is obtained as in Section 2.5.4. The pick-and-place sequence determined by this assignment and the first Hamiltonian path corresponds to a total feeder-board time of 14.10 secs and a total feeder-feeder time of 11.63 secs, for a total travel time of 25.73 secs.

The local improvement procedure is next applied to this initial solution. In each iteration of this procedure, we sequentially consider all feeders, and we attempt to perform one of the exchange steps 1 and 2 on each of them. After four iterations of the procedure, no more improving steps are found. The corresponding feeder-board and feeder-feeder times are respectively 14.68 secs and 8.62 secs, and hence the previous total travel time is improved to 23.30 secs.

Taking this feeder assignment into account, a revised Hamiltonian path with value 14.07 secs is computed. The feeder assignment is in turn modified, resulting (after three iterations of the local improvement procedure) in a total travel time of 22.94 secs. No better Hamiltonian path or assignment are found in the next solutions of subproblems $(E)$ and $(F)$. So, we adopt this solution for machine 2 .

Similar computations are carried out for the other machines. The pick-andplace sequences obtained in this way correspond to processing times of $63.83,66.27$ and 65.82 secs on machines 1,2 and 3 respectively. These times are comparable to the best ones obtained by CQM.

\section{Acknowledgments}

We are grateful to P. van Laarhoven and H. Zijm for introducing us to the problem, and for providing us with the data (CQM, 1988). 


\section{Chapter 3}

\section{A column generation approach to job grouping for flexible manufac- turing systems*}

\subsection{Introduction}

Several authors have stressed the influence of tool management on the overall performance of automated manufacturing facilities in general, and of flexible manufacturing systems (FMS) in particular (Gray et al., 1988; Kiran and Krason, 1988). An FMS consists of a number of numerically controlled machines, linked by automated material handling devices, that perform the operations required to manufacture parts. The tools required by these operations are stored in a limited capacity tool magazine attached to each machine. An automated tool interchanging device enables the machine to interchange tools very quickly (in seconds). This fast tool interchanging capability avoids costly setups while producing with the tools available in the magazine, and is an essential feature of FMSs. When it becomes necessary to add tools to the tool magazine to allow new operations, the machine sometimes has to be shutdown while the tools are interchanged, after which the machine may resume production. The latter type of setup is time-consuming (it may take up to two hours). The performance of an FMS may therefore be considerably boosted by reducing the occurrences of these setups.

An article based on this chapter is submitted for publication. 
In this paper we study a model which aims at minimizing the number of setups. We assume that a number of jobs must be processed on a single machine. The job grouping problem asks for a partition of the jobs into a minimum number of groups (batches), such that the jobs in each group do not require more tools than can be stored in the tool magazine (see Section 3.2 for a precise formulation of the model). This is equivalent to minimizing the number of setups in the situation described above.

The job grouping problem has been studied by different authors, who largely ignore each other. Hirabayashi, Suzuki and Tsuchiya (1984) refer to it as the 'optimal parts grouping problem' and propose a set covering formulation of it. They mention the possibility to solve this set covering formulation using a column generation approach, but concentrate in their paper on developing a branch-and-bound procedure for the column generation subproblem (see Section 3.2). Hwang (1986) investigates the equivalent 'optimal part type grouping problem'. He proposes to solve it approximately by sequentially creating groups that consist of a maximum number of jobs (this is in fact equivalent to solving the set covering formulation of the problem by a greedy heuristic; see Section 3.3). Hwang and Shogan (1989) use branch-and-bound to solve the sequence of subproblems. Hwang (1986) remarks that other sequential approaches (Whitney and Gaul, 1985) and group technology approaches (Chakravarty and Shtub, 1984) exist for part grouping problems, although the latter are inapplicable to FMS because they disregard tool magazine capacity limitations. In Hwang and Shogan (1989) the approach of Hwang (1986) is extended to allow the consideration of due dates. Rajagopalan $(1985 ; 1986)$ gives a general model, which incorporates the job grouping problem as a special case. He presents a number of heuristic procedures for its solution (some of these will be presented in Section 3.3). Stecke and Kim (1988) have extended and made comparisons between the procedures of and Rajagopalan (1985), Whitney and Gaul (1985) and Hwang (1986). Rajagopalan (1985; 1986) and Tang and Denardo (1988b) observe that partitioning jobs into a minimum number of batches can be seen as packing the jobs into a minimum number of bins with fixed capacity. It follows that the bin packing problem is a special case of the job grouping problem, and hence, that the latter is NP-hard (Tang and Denardo, 1988b). Tang and Denardo (1988b) present a non-LP based branch-and-bound procedure for job grouping. They propose non-trivial lower bounds (see Section 3.2), and heuristics similar to Rajagopalan's (see Section 3.3). Kuhn (1990) has developed and tested more heuristics for job grouping. Related problems in process planning are also studied by Kusiak (1985b), Finke and Kusiak (1987) and Bard and Feo (1989). 
In this paper, we implement a column generation approach to solve the linear relaxation of the set covering formulation of the job grouping problem. We demonstrate experimentally that this approach leads to the derivation of extremely strong lower bounds (always equal, in our experiments, to the optimal value of the problem). The column generation scheme is presented in Section 3.2. In Section 3.3, heuristic solution procedures are suggested. The implementation of our procedures is described in Section 3.4. Section 3.5 reviews our computational experiments with these procedures. Section 3.6 contains some conclusions.

\subsection{Lower bounds}

In this section, we present formulations for the job grouping problem and explain the column generation approach we used to derive lower bounds on its optimal value. Some easier, but weaker lower bounds are also discussed.

\subsubsection{The job grouping problem}

The job grouping problem can be described by the following model (Hirabayashi et al., 1984; Hwang, 1986; Tang and Denardo, 1988b). Assume there are $\mathrm{N}$ jobs and $\mathrm{M}$ tools. The basic data are the capacity $\mathrm{C}$ of the tool magazine and the tool requirements for the jobs. These tool requirements are represented by a so-called tool-job matrix $\mathrm{A}$ of dimension $\mathrm{M} \times \mathrm{N}$, with:

$$
\begin{aligned}
\mathrm{a}_{\mathrm{ki}} & =1 \text { if job } \mathrm{i} \text { requires tool } \mathrm{k} \\
& =0 \text { otherwise, }
\end{aligned}
$$

for $\mathrm{k}=1, \ldots, \mathrm{M}$ and $\mathrm{i}=1, \ldots, \mathrm{N}$.

We call a subset (group) $\mathrm{S}$ of jobs (or of columns of $\mathrm{A}$ ) feasible if these jobs together require at most $\mathrm{C}$ tools, i.e. if $\left|\left\{\mathrm{k}: \sum_{\mathrm{i} \in \mathrm{s}} \mathrm{a}_{\mathrm{ki}} \geq 1\right\}\right| \leq \mathrm{C}$. The job grouping problem consists in finding a minimum set of feasible groups such that each job is contained in (at least) one group. To formulate this as a set covering problem, let us suppose that there exist $\mathrm{P}$ feasible groups, and let

$\mathrm{q}_{\mathrm{ij}}=1$ if job $\mathrm{i}$ is contained in the feasible group $\mathrm{j}$, $=0$ otherwise, for $\mathrm{i}=1, \ldots, \mathrm{N}$ and $\mathrm{j}=1, \ldots, \mathrm{P}$.

The job grouping problem is: 


$$
\begin{aligned}
& \text { minimize } \quad \sum_{j=1}^{P} y_{j} \\
& \sum_{j=1}^{p} q_{i j} y_{j} \geq 1 \quad i=1, \ldots, N \\
& y_{j} \geq 0 \quad j=1, \ldots, P \\
& y_{j} \text { integer } \quad \mathrm{j}=1, \ldots, \mathrm{P}
\end{aligned}
$$

where $y_{j}=1$ if group $j$ is part of the optimal covering (notice that $y_{j} \in$ $\{0,1\}$ for $j=1, \ldots, P$ in any optimal solution of (3.1) - (3.4)). Notice that an equivalent set covering model would be obtained if we restricted the set $\{1, \ldots, \mathrm{P}\}$ to the subset of maximal feasible groups, i.e. to those feasible groups of jobs to which no more job can be added without destroying feasibility.

The main drawback of the formulation (3.1) - (3.4) is the possibly huge number of columns that it involves. Several authors report on efficient algorithms for solving large set covering problems to optimality (e.g. Balas and Ho (1980)), or for finding good heuristic solutions to such problems (e.g. Nemhauser and Wolsey (1988) and Vasko and Wolf (1988)). Here, however, even generating the complete set covering formulation is a tedious task for larger instances (see Section 3.5, Table 3.6). In spite of this, we shall see in the next sections that it is possible to solve efficiently the LP-relaxation of (3.1) - (3.4), and that the optimal value of this relaxation provides a very strong lower bound on the optimal value of the set covering. problem. The latter observation can only be seen as an empirical one, without theoretical basis. Indeed, it is known that the LPrelaxation of arbitrary set covering problems can be rather weak. On the other hand:

Theorem 3.1 Any instance of the set covering problem can be interpreted as an instance of the job grouping problem, for some suitable choice of the tool-job incidence matrix and of the capacity.

Proof Consider an arbitrary instance (SC) of the set covering problem, in the form (3.1) - (3.4). We associate with this instance the $\mathrm{P} \times \mathrm{N}$ tool-job matrix A defined by 


$$
a_{j i}=1-q_{i j} \quad j=1, \ldots, P ; i=1, \ldots, N
$$

and the capacity $\mathrm{C}=\mathrm{P}-1$ (we assume without loss of generality that $Q=\left(q_{i j}\right)$ has no zero row, so that $A$ has no column involving $\mathrm{C}+1$ ones). We claim that the set covering formulation of the job grouping instance described by $\mathrm{A}$ and $\mathrm{C}$ is exactly (SC). Indeed, a subset $S$ of jobs $(S \subseteq N$ ) is feasible for the instances described by $(A, C)$ if and only if there exists a row $j$ of $A(j \in\{1, \ldots, P\})$ such that $a_{j i}=0$ for all $i \in S$, or, equivalently, if and only if there is a column $j$ of $\left(q_{i j}\right)$ such that $q_{i j}=1$ for all $i \in S$. But this also means that the (maximal) columns of $\left(\mathrm{q}_{\mathrm{ij}}\right)$ exactly correspond to the maximal feasible sets of jobs.

Notice, however, that the value of the tool magazine capacity occurring in this proof (namely, the total number of tools minus one) is not very realistic from the viewpoint of the job grouping problem.

\subsubsection{Column generation}

To find a lower bound for the set covering problem, we want to solve the LP-relaxation of (3.1) - (3.4), i.e. the problem (3.1) - (3.3). We avoid the difficulty of explicitly generating all columns of this problem, by working with only a subset of the columns and adding new columns as needed. This approach was suggested by Gilmore and Gomory (1961) for solving cutting stock problems. It can also be seen as an essential part of the DantzigWolfe decomposition (Dantzig and Wolfe, 1960). For a thorough discussion of column generation we point to Chvátal (1983), and we only briefly recall here the main features of the approach.

At each iteration of the column generation procedure, we solve the LP obtained by restricting (3.1) - (3.3) to some subset $T$ of columns, i.e. we solve a problem of the form:

$$
\begin{array}{lll}
\text { minimize } & \sum_{j \in T} y_{j} & \\
\text { subject to } & \sum_{j \in T} q_{i j} y_{j} \geq 1 & i=1, \ldots, N \\
& y_{j} \geq 0 & j \in T
\end{array}
$$

for some $\mathrm{T} \subseteq\{1, \ldots, \mathrm{P}\}$ (we shall indicate in Section 3.4 how an initial 
set $\mathrm{T}$ may be chosen). Let $\mathrm{y}^{*}$ be an optimal solution to (3.5) - (3.7) and $\lambda^{*}$ be an optimal solution to the dual of (3.5) - (3.7). Consider also the dual of (3.1) - (3.3), in the form

$$
\operatorname{maximize} \quad \sum_{i=1}^{N} \lambda_{i}
$$

$$
\begin{array}{ll}
\text { subject to } & \sum_{i=1}^{N} q_{i j} \lambda_{i} \leq 1 \\
\lambda_{i} \geq 0 & i=1, \ldots, P
\end{array}
$$

Observe that $\mathrm{y}^{*}$ satisfies the constraints (3.2), (3.3) and that $\sum_{\mathrm{N}}^{\mathrm{P}} \mathrm{P}_{\mathrm{j}} \mathrm{y}_{\mathrm{j}}^{*}=$ $\sum_{i=1}^{N} \lambda_{i}^{*}$ (we suppose here that $y_{j}^{*}$ is extended to a vector of $\mathbb{R}^{P^{j}}$ by letting $y_{j}=0$ for $j \notin T$ ). Hence, if $\lambda$ satisfies all constraints (3.9), it follows from the duality theorem of linear programming theory (see Chvátal (1983)) that $y^{*}$ is an optimal solution to the LP relaxation (3.1) - (3.3). In such a case, the column generation procedure does not need to proceed further. On the other hand, if $\lambda^{*}$ does not satisfy (3.9), that is if there exists a column $j \in\{1, \ldots, P\}$ such that $\sum_{i=1}^{N} q_{i j} \lambda_{i}^{*}>1$, then the current set $T$ can be extended by this new index $j$, and a new iteration of the column generation procedure can be started (alternatively, $j$ can be seen as a column with negative reduced cost for the relaxation (3.1) - (3.3)). Classical LP theory ensures again that this procedure can be made to converge in a finite number of iterations. In the next subsection, we discuss the question of finding a violated constraint among (3.9).

\subsubsection{The generation subproblem}

The efficiency of column generation procedures is to a large extend determined by the complexity of the so-called generation subproblem, that is, in our case of the subproblem:

given $\lambda_{l}^{*}, \ldots, \lambda_{N}^{*}$, is there $j \in\{1, \ldots, P\}$ such that $\sum_{i=1}^{N} q_{i j} \lambda_{i}^{*}>1 \cdot ?(3.11)$

In many successful applications of column generation, the subproblem is relatively easy, e.g. solvable in polynomial or pseudo-polynomial time (see e.g. Gilmore and Gomory (1961), Desrosiers, Soumis and Desrochers (1984), Ribeiro, Minoux and Penna (1989), and Minoux (1987) for a 
general discussion). Other applications exist, however, where the subproblem itself turns out to be hard (see e.g. Kavvadias and Papadimitriou (1989), Jaumard, Hansen and Poggi de Aragão (1991)). In order to determine the complexity of our subproblem, notice first that (3.11) can be rephrased as:

given $\lambda_{1}^{*}, \ldots, \lambda_{N}^{*}$, is there a feasible group $S$ such that $\sum_{i \in S} \lambda_{i}^{*}>1$ ? (3.12)

Now, (3.12) could certainly be answered if we could find a feasible group $S$ which maximizes the expression $\sum_{i \in S} \lambda_{i}^{*}$ over all feasible groups. We may express this reformulation of the subproblem as follows. Introduce variables

$$
\begin{array}{rll}
x_{i}=1 & & \text { if job } \mathrm{i} \text { is in group } \mathrm{S} \\
& =0 &
\end{array}
$$

for $\mathrm{i}=1, \ldots, \mathrm{N}$, and

$$
\begin{aligned}
z_{k} & =1 & & \text { if tool } \mathrm{k} \text { is required by some job in } S, \\
& =0 & & \text { otherwise, }
\end{aligned}
$$

for $\mathrm{k}=1, \ldots, \mathrm{M}$. The maximization version of (3.12) becomes (Hirabayashi et al., 1984):

$\operatorname{maximize} \quad \sum_{i=1}^{N} \lambda_{i}^{*} x_{i}$

subject to

$$
\begin{array}{ll}
a_{k i} x_{i} \leq z_{k} & \begin{array}{l}
i=1, \ldots, N \\
k=1, \ldots, M
\end{array} \\
\sum_{k=1}^{M} z_{k} \leq C & \\
x_{i} \in\{0,1\} & i=1, \ldots, N \\
z_{k} \in\{0,1\} & k=1, \ldots, M
\end{array}
$$

This problem is known to be $N P$-hard, even when $\lambda_{1}^{*}=\ldots=\lambda_{\mathrm{N}}^{*}=1$ (Gallo, Hammer and Simeone, 1980). Notice that, when $\lambda_{1}^{*}=\ldots=\lambda_{N}^{*}=$ 1, (3.13) - (3.17) boils down to determining a feasible group that contains as many jobs as possible; this subproblem has been considered by Hwang (1986) and Hwang and Shogan (1989). Problem (3.13) - (3.17) (and generalizations thereof) has been investigated by a number of authors. Hirabayashi et al. (1984) developed a branch-and-bound procedure for it. 
To obtain an upper bound, they solve the linear relaxation of the problem by a specialized primal-dual algorithm. Mamer and Shogan (1987) use a Lagrangian method with the help of subgradient optimization to solve the relaxation of (3.13) - (3.17). This approach has been developed further by Gallo and Simeone (1988) (see also Chaillou, Hansen and Mahieu (1989)). Dietrich, Lee and Lee (1991) present a heuristic procedure for the problem (see Section 3.4). They also use the LP-relaxation for obtaining an upper bound, and present some valid inequalities to improve this bound and to fasten up the branch-and-bound search.

From a practical viewpoint, (3.13) - (3.17) remains a hard problem to solve. In particular, experimental results of Dietrich et al. (1991) show a large gap between the LP-relaxation value and the optimal value of (3.13) (3.17). Our own experience also indicates that the LP-relaxation is generally weak. Especially in the case where optimality in the column generation procedure is nearly reached (i.e. where the generation subproblem has an optimal value close to 1), the gap between LP- and IPformulation is considerable (often larger than 2). This results in large search trees when attacking (3.13) - (3.17) by branch-and-bound. Another drawback of solving (3.13) - (3.17) to optimality is that this only allows one new column of the set covering problem to be generated in each iteration (i.e., we find only one violated inequality of type (3.9)). This may lead to a large number of iterations of the column generation procedure. Because we are using the LP package LINDO in our experiments, and this package does not allow to add columns to a model in a dynamic fashion, one new LP problem has to be re-optimized from scratch in each such iteration, a rather costly operation.

In view of all these considerations, we decided to use a complete enumeration procedure for the solution of the generation subproblem. Thus, in terms of the formulation (3.12), we are not only interested in finding one group $S$ for which $\sum_{i \in S} \lambda_{i}^{*}>1$, but in finding all (or many) such groups. All the corresponding columns may enter the set covering formulation, and this tends to reduce the number of iterations of the column generation procedure.

The enumeration procedure works as follows. First, we sort the dual variables by nonincreasing values, say e.g. $\lambda_{1}^{*} \geq \lambda_{2}^{*} \geq \ldots \geq \lambda_{\mathrm{N}}^{*}$. Then we grow a binary tree, by successively attempting to include or not to include each of the jobs $1,2, \ldots, N$ in a feasible group. Early on in the column generation procedure, the $\lambda_{i}^{*}$ 's are bad estimates of the optimal dual variables, and hence the enumeration procedure produces very quickly a large number of feasible groups $S$ with $\sum_{i \in S} \lambda_{i}^{*}>1$. Therefore, the total number of columns that is generated in one iteration is limited to a fixed arbitrary number (100 in our implementation). For the instance sizes which 
we considered in our experiments, the enumeration procedure always remained manageable (see Section 3.5).

\subsubsection{Computation of lower bounds via column generation}

The column generation procedure can be summarized as follows (see Section 3.4 for details about its implementation):

\begin{tabular}{|c|c|}
\hline Initialisation & $\begin{array}{l}\text { Generate an initial set } T \text { of columns of the set } \\
\text { covering formulation }(3.1)-(3.3) \text {. }\end{array}$ \\
\hline LP Solution & $\begin{array}{l}\text { Solve the LP }(3.5)-(3.7) \text {; let } y^{*} \text { and } \lambda^{*} \text { be } \\
\text { optimal primal and dual solutions of }(3.5) \text { - } \\
(3.7) \text {. }\end{array}$ \\
\hline olumn Generation & $\begin{array}{l}\text { Generate new columns by solving the } \\
\text { generation subproblem: that is, find indices } \\
j \in\{1, \ldots, P\} \text { such that } \sum_{i=1}^{N} q_{i j} \lambda_{i}^{*}>1 \text {, } \\
\text { and let } T \leftarrow T \cup\{j\} \text { for each such } j \text {. } \\
\text { If no such new columns can be found then } \\
\text { STOP: } y^{*} \text { is an optimal solution of }(3.1)- \\
\text { (3.3); otherwise return to LP Solution. }\end{array}$ \\
\hline
\end{tabular}

When the column generation procedure stops we have an optimal solution $\mathrm{y}^{*}$ for the LP relaxation (3.1) - (3.3). Rounding up the solution value $\sum_{\mathrm{j} \in \mathrm{T}}$ $y_{j}^{*}$ to the next integer gives a lower bound for the job grouping problem. We will refer to the bound $\left\lceil\sum_{j \in T} y_{j}^{*}\right\rceil$ as $\mathrm{LB}_{\mathrm{CG}}$.

It is also possible to compute weaker lower bounds on the optimal value of the job grouping problem before the column generation procedure terminates. To see this, let $\mathrm{Z}$ denote the optimal value of the generation subproblem (3.13) - (3.17), as computed for instance in some iteration of the column generation step (for the results below to be valid, the $\lambda_{i}^{*}$ may actually be arbitrary numbers in $[0,1]$, and do not necessarily need to arise from the LP solution step). Farley (1990) observed the following:

Theorem 3.2 If $\mathrm{Z} \geq 1$, then $\sum_{\mathrm{i}=1}^{N} \lambda_{\mathrm{i}}^{*} / \mathrm{Z}$ is a lower bound on the optimal value of (3.1) - (3.3).

Proof: By definition, $\mathrm{Z}$ is the maximum value of $\sum_{\mathrm{i}=1}^{\mathrm{N}} \mathrm{q}_{\mathrm{ij}} \lambda_{\mathrm{i}}^{*}$ over $\mathrm{j}$ (see (3.11)). Thus, $\lambda^{*} / \mathrm{Z}$ is a feasible solution for the dual (3.8) $-(3.10)$ of $(3.1)-(3.3)$, and it follows that $\sum_{i=1}^{N} \lambda_{i}^{*} / Z$ 
is a valid lower bound for (3.1) - (3.3).

Another lower bound can also be derived as follows:

Theorem 3.3 If $Z \geq 1$, then $\sum_{i=1}^{N} \lambda_{i}^{*}+N \cdot(1-Z)$ is a lower bound on the optimal value of (3.1) - (3.3).

Proof: Let $\mathrm{y}^{*}$ be an optimal solution of (3.1) - (3.3). Notice that $\sum_{i=1}^{N} q_{i j} y_{j}^{*} \geq 1(i=1, \ldots, N)$ and $\sum_{j=1}^{P} y_{j}^{*} \leq N$. Hence, $\sum_{\mathrm{j}=1}^{\mathrm{P}} \mathrm{y}_{\mathrm{j}}^{*} \geq \sum_{\mathrm{j}=1}^{\mathrm{P}} \mathrm{y}_{\mathrm{i}}^{*}+\sum_{\mathrm{i}=1}^{\mathrm{N}}\left(1-\sum_{\mathrm{j}=1}^{\mathrm{P}=1} \mathrm{q}_{\mathrm{ij}} \mathrm{y}_{\mathrm{j}}^{*}\right) \lambda_{\mathrm{i}}^{*}$

$=\quad \sum_{i=1}^{N} \lambda_{i}^{*}+\sum_{j=1}^{P}\left(1-\sum_{i=1}^{N} q_{i j} \lambda_{i}^{*}\right) y_{j}^{*}$

$\geq \quad \sum_{i=1}^{N} \lambda_{i}^{*}+(1-Z) \sum_{j=1}^{p} y_{j}^{*}$

$\geq \quad \sum_{i=1}^{N} \lambda_{i}^{*}+N(1-Z)$.

Theorem 3.4 If $Z>1$, then $\sum_{i=1}^{N} \lambda_{i}^{*} / Z>\sum_{i=1}^{N} \lambda_{i}^{*}+N \cdot(1-Z)$.

Proof: Trivial.

Theorem 3.4 shows that the bound given in Theorem 3.3 is strictly better than the bound in Theorem 3.2 whenever $\mathrm{Z}>1$. When $\mathrm{Z}=1$, both bounds coincide with the optimal value of (3.1) - (3.3). Thus, we will only consider from now on the stronger bound $\sum_{i=1}^{N} \lambda_{i}^{*} / Z$. More precisely, we define

$$
\mathrm{LB}_{\text {Farley }}=\left\lceil\sum_{\mathrm{i}=1}^{\mathrm{N}} \lambda_{\mathrm{i}}^{*} / \mathrm{Z}\right\rceil
$$

$\mathrm{LB}_{\text {Farley }}$ is obviously a valid lower bound on the optimal value of the job grouping problem. As the column generation proceeds, $\mathrm{Z}$ approaches 1 and $\mathrm{LB}_{\text {Farley }}$ approaches the lower bound $\mathrm{LB}_{\mathrm{CG}}$ (see Farley (1990)).

\subsubsection{Lagrangian relaxation}

In this subsection, we present an alternative integer programming model for the job grouping problem and we discuss the quality of the bounds that it yields, either by continuous relaxation or by Lagrangian relaxation.

In this model, a variable $x_{i j}$ is used to denote the assignment of job $i$ to one of $\mathrm{N}$ groups, indexed by $\mathrm{j}(\mathrm{i}=1, \ldots, \mathrm{N} ; \mathrm{j}=1, \ldots, \mathrm{N}$ ) (one may best 
think of the $\mathrm{N}$ groups as being initially empty).

We use the following notation

$\mathrm{x}_{\mathrm{ij}}=1$ if job $\mathrm{i}$ is assigned to group $\mathrm{j}$,

$=0$ otherwise,

$\mathrm{y}_{\mathrm{j}}=1$ if group $\mathrm{j}$ is non-empty,

$=0$ otherwise,

$\mathrm{z}_{\mathrm{kj}}=1$ if tool $\mathrm{k}$ is used for the production of group $\mathrm{j}$, $=0$ otherwise,

for $\mathrm{i}=1, \ldots, \mathrm{N}, \mathrm{j}=1, \ldots, \mathrm{N}, \mathrm{k}=1, \ldots, \mathrm{M}$.

The model is now:

$\operatorname{minimize} \quad \sum_{j=1}^{N} y_{j}$

subject to $\quad \sum_{j=1}^{N} x_{i j}=1 \quad i=1, \ldots, N$

$$
a_{k i} x_{i j} \leq z_{k j}
$$$$
\mathrm{i}=1, \ldots, \mathrm{N}
$$$$
\mathrm{j}=1, \ldots, \mathrm{N}
$$$$
\mathrm{k}=1, \ldots, \mathrm{M}
$$

$$
\sum_{k=1}^{M} z_{k j} \leq C y_{j}
$$$$
\mathrm{j}=1, \ldots, \mathrm{N}
$$$$
y_{j} \in\{0,1\}
$$$$
\mathrm{j}=1, \ldots, \mathrm{N}
$$$$
\mathrm{x}_{\mathrm{ij}} \in\{0,1\}
$$$$
\mathrm{i}=1, \ldots, \mathrm{N}
$$$$
\mathrm{j}=1, \ldots, \mathrm{N}
$$$$
\mathrm{z}_{\mathrm{kj}} \in\{0,1\}
$$$$
\begin{aligned}
& \mathrm{j}=1, \ldots, \mathrm{N} \\
& \mathrm{k}=1, \ldots, \mathrm{M}
\end{aligned}
$$

The objective function (3.18) minimizes the number of nonempty groups. Restrictions (3.19) make sure that each job is assigned to some group. Restrictions (3.20) assure that the tools needed for a job are available for the production of the group to which the job is assigned. Restrictions (3.21) describe the tool magazine capacity constraints for each group. 
The continuous relaxation of this model yields a weak lower bound on the optimal value. Indeed, the solution $x_{i j}=1 / N, z_{k j}=1 / N$ and $y_{j}=M / C N$ $(i=1, \ldots, N ; j=1, \ldots, N ; k=1, \ldots, M)$ is feasible, with an objective function value of $\mathrm{M} / \mathrm{C}$ (which is trivially a valid lower bound; see Section 3.2.6).

Lagrangian relaxation could be used to compute a stronger bound. For instance, if we dualize restrictions (3.19) with multipliers $\lambda_{1}, \ldots, \lambda_{N}$, we obtain a lower bound $\mathrm{LB}_{\mathrm{LR}}(\lambda)$ by solving:

$\operatorname{LB}_{\mathrm{LR}}(\lambda)=\operatorname{minimize} \sum_{j=1}^{N} y_{j}+\sum_{i=1}^{N} \lambda_{i}\left(1-\sum_{j=1}^{N} x_{i j}\right)$

subject to

$(3.20)-(3.24)$.

Up to deletion of an additive constant, $\sum_{i=1}^{N} \lambda_{i}$, this problem can be equivalently rewritten as

$\operatorname{minimize} \quad \sum_{j=1}^{N}\left(y_{j}-\sum_{i=1}^{N} \lambda_{i} x_{i j}\right)$

subject to

$(3.20)-(3.24)$

Now problem (3.18'), (3.20) - (3.24) can be decomposed into $\mathrm{N}$ identical subproblems, one for each value of $j=1, \ldots, N$. Deleting the index $j$, the generic form of each subproblem is:

minimize

$$
y-\sum_{i=1}^{N} \lambda_{i} x_{i}
$$

subject to

$$
\begin{array}{ll}
\mathrm{a}_{\mathrm{ki}} \mathrm{x}_{\mathrm{i}} \leq \mathrm{z}_{\mathrm{k}} & \mathrm{i}=1, \ldots, \mathrm{N} ; \\
\mathrm{k}=1, \ldots, \mathrm{M}
\end{array}
$$




$$
\begin{array}{lll}
\mathrm{y} & \in\{0,1\} & \\
\mathrm{x}_{\mathrm{i}} \in\{0,1\} & \mathrm{i}=1, \ldots, \mathrm{N} \\
\mathrm{z}_{\mathrm{k}} \in\{0,1\} & \mathrm{k}=1, \ldots, \mathrm{M}
\end{array}
$$

If $y=0$ in the optimal solution of (3.25) - (3.30), then also $z_{k}=0$ for $k$ $=1, \ldots, M, x_{i}=0$ for $i=1, \ldots, N$, and the optimal value is 0 . If $y=1$ at optimality, then minimizing the objective function (3.25) becomes equivalent to maximizing $\sum_{i=1}^{N} \lambda_{i} x_{i}$. Therefore, we conclude that the subproblem arising via this Lagrangian relaxation is essentially equivalent to the subproblem (3.13) - (3.17) arising via column generation.

Denote by $\mathrm{Z}$, as usual, the maximum of $\sum_{\mathrm{i}=1}^{\mathrm{N}} \lambda_{\mathrm{i}} \mathrm{x}_{\mathrm{i}}$ under the constraints (3.26) - (3.30). The previous discussion shows that the optimal value of (3.25) - (3.30) is equal to $\min (0,1-\mathrm{Z})$. This in turn implies that the lower bounds $\operatorname{LB}_{\mathrm{LR}}(\lambda)$, computed from (3.18'), (3.20) - (3.24), is equal to $\sum_{i=1}^{N} \lambda_{i}+N \cdot \min (0,1-Z)$. As we already know, this bound is weaker than $\mathrm{LB}_{\text {Farley }}$ for all $\lambda$ such that $Z>1$ (see Theorem 3.4), and coincides with the optimal value of (3.1) - (3.3) when $Z \leq 1$. Thus, the Lagrangian relaxation approach described here does not yield better bounds than the column generation procedure.

Observe that a "best possible" choice of the multipliers $\lambda_{1}, \ldots, \lambda_{N}$, i.e. one leading to the maximum value of $\operatorname{LB}_{\mathrm{LR}}(\lambda)$, could be searched for by a subgradient optimization procedure (Fisher, 1981) or by a multiplier adjustment procedure (Fisher, Jaikumar and Van Wassenhove, 1986). The column generation procedure can also be seen as using an LP-solver to adjust the values of the multipliers. The Lagrangian relaxation approach will not be considered any further in this work.

\subsubsection{Other lower bounds}

We discuss in this subsection some more lower bounds for the job grouping problem. By duality, the optimal value of the problem (3.8) - (3.10) is equal to the optimal value of $(3.1)$ - (3.3), i.e. (up to rounding) $\mathrm{LB}_{\mathrm{CG}}$. Thus, the optimal value of (3.8) - (3.10) under the additional restriction

$$
\lambda_{\mathrm{i}} \in\{0,1\} \quad \mathrm{i}=1, \ldots, \mathrm{N}
$$

is a lower bound on $\mathrm{LB}_{\mathrm{CG}}$; we denote it by $\mathrm{LB}_{\mathrm{SP}}$. This lower bound can be interpreted as follows. Call two jobs compatible if they form a feasible group and incomparible otherwise. Then, $\mathrm{LB}_{\mathrm{SP}}$ is nothing but the 
maximum number of pairwise incompatible jobs. The problem (3.8) $(3.10),(3.31)$ is a so-called set packing problem. Conversely, a construction similar to the one used for Theorem 3.1 shows that any instance of the set packing problem can arise in that way. It follows from this observation that computing $\mathrm{LB}_{\mathrm{SP}}$ is $N P$-hard (see e.g. Nemhauser and Wolsey (1988) p. 117).

Tang and Denardo (1988b) propose a lower bound which is also based on the concept of compatibility of jobs. In their so-called sweeping procedure, they sequentially create a number of groups as follows. In each step of the procedure, they first select a job (seed) which is compatible with the fewest number of other (not yet selected) jobs (in case of a tie, the job for which the set of compatible jobs requires the smallest number of tools is selected). Next, the seed, along with all jobs which are compatible with it, are selected to form one group. The procedure is repeated until all jobs have been selected. The number of groups so created, say L (i.e., the number of steps of the sweeping procedure) is a valid lower bound for the job grouping problem. In fact, $\mathrm{L}$ can best be seen as a lower bound on $\mathrm{LB}_{S P}$, since the seeds are mutually incompatible, and hence define a feasible solution of the set packing problem (3.8) - (3.10), (3.31). From this viewpoint, the sweeping procedure is a greedy heuristic applied to (3.8) (3.10), (3.31).

Tang and Denardo (1988b) also point to the lower bound $\lceil\mathrm{M} / \mathrm{C}\rceil$. Combining this bound with $\mathrm{L}$ yields the lower bound $\mathrm{LB}_{\mathrm{SW}}=$ $\max \{\lceil\mathrm{M} / \mathrm{C}\rceil, \mathrm{L}\}$ (Tang and Denardo, 1988b).

The lower bound $\mathrm{LB}_{S W}$ can be further improved by "incorporating" the lower bound $\lceil\mathrm{M} / \mathrm{C}\rceil$ in the sweeping procedure. More precisely, a lower bound for the job grouping problem can be calculated in each step of the sweeping procedure by summing the number of already created groups by the sweeping procedure and the lower bound $\left\lceil\left|\cup_{i \in I} T_{i}\right| / C\right\rceil$, where I is the set of "not yet selected" jobs, and $T_{i}$ is the set of tools needed by job $\mathrm{i}$. This procedure generates a sequence of valid lower bounds, the first of which is equal to $\lceil\mathrm{M} / \mathrm{C}\rceil$ and the last of which is equal to $\mathrm{L}$. We refer to this procedure as the "modified sweeping procedure". It yields a new lower bound, equal to the maximum of the bounds in the sequence, which we denote by $\mathrm{LB}_{\mathrm{MSW}}$.

We have considered a number of lower bounds for the job grouping problem. Summarizing we have: 


$$
\begin{array}{ll}
\mathrm{LB}_{\text {Farley }} \leq \mathrm{LB} \text { CG } & \text { (see Section 3.2.4) } \\
\mathrm{LB}_{\mathrm{LR}} \leq \mathrm{LB}_{\text {Farley }} & \text { (see Section 3.2.5) } \\
\mathrm{LB}_{\mathrm{SP}} \leq \mathrm{LB}_{\mathrm{CG}} & \text { (Duality) } \\
\mathrm{LB}_{\mathrm{SW}} \leq \mathrm{LB} \text { MSW } & \text { (see this section) }
\end{array}
$$

In our implementation we use the bound $\mathrm{LB}_{\mathrm{MSW}}$ for its computational simplicity and the lower bounds $\mathrm{LB}_{\text {Farley }}$ and $\mathrm{LB}_{\mathrm{CG}}$ if $\mathrm{LB}_{\mathrm{MSW}}$ is not strong enough.

\subsection{Upper bounds}

In this section a number of heuristic methods will be described to compute good solutions for the job grouping problem and hence upper bounds on its optimal value. First, we will describe a number of procedures that sequentially build groups. The second part will discuss procedures based on solving the set covering formulation.

\subsubsection{Sequential heuristics for grouping}

Sequential heuristic procedures use a two-step approach for building each group. In the first step, a job is picked to be used as a seed. Unless explained otherwise, we always pick a job that requires the highest number of tools. Then a selection rule is used to add jobs to the group until the tool magazine capacity constraint prohibits the addition of any other job to this group. The two-step procedure is repeated until all jobs are assigned to some group. For selecting the next job to be assigned to a group (in step 2) a number of different rules have been considered. We now describe them. For a group $S$ and a job $i \notin S$, let

$\mathrm{t}_{\mathrm{i}}=$ number of tools required by job $\mathrm{i}$;

$b_{i}=$ number of tools required both by job $\mathrm{i}$ and by some job already in $\mathrm{S}$.

1. MIMU rule

Tang and Denardo (1988b) select the job that has the largest number of tools in common with the jobs already in the group. In case of a tie, the job which requires the smallest number of additional tools is selected. The procedure is called Maximal Intersection Minimal Union. (Maximize $b_{i} ;$ in case of a tie minimize $t_{i}$ )

2. MI rule

This is the rule obtained if only the first part of the MIMU rule is used, and ties are arbitrarily broken. (Maximize $b_{i}$ ) 
3. $M U$ rule

It is also possible to select jobs according only to the Minimal Union criterion: select the job that requires a minimum number of additional tools. Minimize $\left.\left(t_{i}-b_{i}\right)\right)$

4. Whitney and Gaul rule

Whitney and Gaul (1985) favour jobs that bring with them a large number of versatile tools. This idea is operationalized by selecting a job for which the ratio $\left(b_{i}+1\right) /\left(t_{i}+1\right)$ is maximal. (Maximize $\left.\left(b_{i}+1\right) /\left(t_{i}+1\right)\right)$

\section{Rajagopalan rule}

Rajagopalan (1985) proposes a number of procedures based on the First Fit Decreasing rule for bin-packing. Among these, we consider one that first assigns weights to the tools and then selects the job that requires the most 'expensive' tools. More precisely, each tool k receives a weight $a_{k}$ equal to the number of jobs that require tool $k$ among the jobs that still have to be assigned to a group. Then, the priority of job $i$ is calculated by summing the weights $a_{k}$ of the tools that must be added to the tool magazine in case job $\mathrm{i}$ is assigned to the group. The job with the largest priority is selected first. For this rule, the first job in each group (seed) is also selected according to the same criterion.

6. Modified Rajagopalan rule

The procedure of Rajagopalan (1985) can be changed in the following way: the weight $a_{k}$ for each tool $k$ is defined as the number of jobs that require tool $\mathrm{k}$ among the jobs already selected in the group. The priority of a job is the sum of the weights of the tools that are needed for that job. The job with the highest priority is selected.

7. Marginal gain rule

The addition of job i to a group usually requires that extra tools be loaded in the tool magazine. This new tool configuration may in turn allow the execution of other, not yet selected, jobs; denote by $\mathrm{p}_{\mathrm{i}}$ the number of such jobs. This rule selects a job $i$ that maximizes $\mathrm{p}_{\mathrm{i}}$ (a similar idea is used by Dietrich et al. (1991)).

\subsubsection{Set covering heuristics}

In the course of the column generation procedure, several set covering 
subproblems of type (3.5) - (3.7) are formulated. Each such subproblem can be viewed as an approximation of the complete formulation (3.1) (3.4). In particular, each feasible solution of the system (3.6) - (3.7) is a feasible solution of (3.2) - (3.3), and hence each 0-1 solution of (3.6) (3.7) defines a heuristic solution to the job grouping problem. We have used this observation in various ways.

First, the solution of (3.5) - (3.7) found by LINDO during the column generation procedure sometimes happens to be a $0-1$ solution which improves upon the current best solution. Such solutions can be detected with very little additional computational effort and may avoid the use of other upper bounding procedures.

It is also possible to systematically generate "good" $0-1$ solutions of the subproblem (3.5) - (3.7). This can be done using either a heuristic procedure or an exact algorithm. We have considered both possibilities. As a heuristic, we used the well-known greedy procedure (Nemhauser and Wolsey, 1988 p. 466); this constructive heuristic recursively selects as next group (column) one which contains a maximum number of jobs, until all jobs are included in some group (i.e. are covered by some column).

Alternatively, subproblem (3.5) - (3.7) could also be solved to optimality in $0-1$ variables, by relying on the capability of LINDO to handle integer programming problems. In view of the computational burden involved in this approach, we chose to turn it into a heuristic by requiring only a small number of variables to be integer. We only used this heuristic when the column generation procedure ended without an optimal solution. We will il explain in Section 3.4 the implementational details of this approach.

\subsection{Implementation}

In Sections 2 and 3, an overview has been given of the methods that can be used for obtaining lower bounds and upper bounds for the job grouping problem: Also, the general principle of the column generation procedure and the difficulty of solving the generation problem have been discussed. Now, we focus on implementational issues. The procedure that we implemented consists of four main steps. We first briefly sketch the whole procedure before commenting on each individual step.

Step I: Use the heuristics of Section 3.3.1. to produce a first upper bound.

Compute the simple lower bounds $\mathrm{LB}_{\mathrm{SW}}$ and $\mathrm{LB}_{\mathrm{MSW}}$.

If optimality is achieved then STOP 
Otherwise construct an initial set covering formulation using the groups that have been generated using the heuristic procedures.

Step II: Use the greedy heuristic to solve the initial set covering formulation.

If optimality is achieved then STOP.

Otherwise use a heuristic to add a number of columns to the initial formulation.

Solve again the resulting set covering formulation using the greedy procedure. If optimality is achieved then STOP.

Step III: Solve the LP-relaxation of the current formulation.

Check whether the primal solution is integral and whether its value improves the current upper bound.

Use the dual variables to formulate the generation subproblem and generate new columns with negative reduced cost.

Calculate $\mathrm{LB}_{\text {Farley. }}$. If optimality is achieved then STOP.

If no columns with negative reduced cost have been found, then continue with Step IV.

Otherwise, update the set covering formulation and repeat Step III.

Step IV: Use the last set covering formulation for finding an improved heuristic solution.

In Step I an upper bound is obtained by using the 7 heuristics of Section 3.3.1 and retaining the best solution. A lower bound is obtained by calculating the bounds $\mathrm{LB}_{S W}$ and $\mathrm{LB}_{\mathrm{MSW}}$ of Section 3.2.6. If the lower bound equals the upper bound, the procedure stops and steps II-IV are not necessary. Otherwise the groups generated by the heuristics are used to generate an initial set covering formulation of the problem.

Step II aims at improving the initial formulation and the current upper bound before starting the column generation procedure. The first set covering formulation is solved using the greedy heuristic (Section 3.3.2.). If optimality is not established yet, then a heuristic based on the work of Dietrich et al. (1991) is used for generating additional columns as follows. Each job is considered as a seed, around which a group is built by iteratively adding that job $\mathrm{i}$ for which the ratio $\mathrm{p}_{\mathrm{i}} /\left(\mathrm{t}_{\mathrm{i}}-\mathrm{b}_{\mathrm{i}}\right)$ is maximal, where $\left(t_{i}-b_{i}\right)$ is the number of additional tools needed for job $i$ and $p_{i}$ is the number of additional jobs that may be executed with the new set of tools in the tool magazine (see Section 3.3.1). In this way $N$ (number of jobs) new groups (i.e. columns) are constructed and used to extend the set covering formulation. This new formulation is solved again using the 
greedy heuristic. Notice that the second part of step II is time consuming (see Section 3.5.2); this is the main reason why we first apply the greedy heuristic to the initial formulation rather than directly extending this formulation.

The third step is critical to the procedure. First, the LP-relaxation of the current set covering formulation is solved using the linear programming package LINDO. The primal and dual solutions are stored, and the primal solution is checked for integrality. If it is integral and involves fewer groups than the current best solution, then its value is stored as a new upper bound. The dual variables are then used in the generation subproblem. This problem is solved using the enumeration strategy described in Section 3.2.3. In the first steps of the column generation procedure only a limited enumeration takes place because of (the selfimposed) maximum of 100 columns that may be generated by the enumeration procedure. When a complete enumeration is performed, the optimal value $\mathrm{Z}$ of the generation subproblem is used for computing the

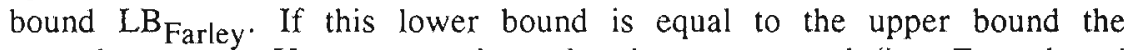
procedure stops. If no new column has been generated (i.e. $\mathrm{Z}=1$ and $\mathrm{LB}_{\text {Farley }}=\mathrm{LB}_{\mathrm{CG}}$, then the column generation subroutine terminates, and we continue with step IV. Otherwise, the new columns are added to the set covering formulation. Also, to limit the size of the formulation, all columns with a small reduced cost are eliminated. More precisely, columns for which $\sum_{i=1}^{N} \mathrm{q}_{\mathrm{ij}} \lambda_{\mathrm{i}}<1-\alpha$ are removed from the formulation, where $\alpha$ is an arbitrary chosen parameter $(\alpha=0.25$ in our implementation). This may cause the procedure to cycle, as columns are removed from the formulation, then enter it again, etc. In our tests (with $\alpha=0.25$ ) cycling occurred for 4 instances out of 550 , but could be avoided when the procedure was run anew with $\alpha$ set to a larger value.

When there is still a gap between the upper and lower bound generated in Steps I-III, more work has to be done. A branch-and-bound procedure could be used to establish optimality. However, it is also possible to use the last set covering formulation to improve the upper bound, as we explained in Section 3.3.2. In our implementation, we first solve this formulation by the greedy heuristic. If this is not effective, we solve a slightly modified set covering formulation with LINDO, requiring only a limited number of variables to take $0-1$ values. More precisely, the $T$ variables which assume the largest value in the continuous solution of the set covering formulation (where columns for which $\sum_{i=1}^{N} q_{i j} \lambda_{i}<1-\beta$ are removed to limit the size of the formulation, with $\beta=0.10$ ), extended by the additional constraint $\sum_{j=1}^{P} y_{j} \geq{ }^{L B} C G$, are forced to be integer. 
The parameter $\mathrm{T}$ is taken equal to $\mathrm{LB}_{\mathrm{CG}}+5$ if the number of columns is smaller than 50 (resp. $\mathrm{LB}_{\mathrm{CG}}+15$ if the number of columns is between 50 and 150 , and $\mathrm{LB}_{\mathrm{CG}}+25$ otherwise). Because of the small number of integer variables, the resulting mixed $0-1$ problem is easily solved by branch-and-bound.

Notice that the choices made for the various parameters of the procedure (maximum number of columns generated in each iteration, $\alpha, \mathrm{T}, \beta$ ) influence the sequence of LP subproblems generated, and hence also the heuristic solutions produced in Steps III and IV. These choices may sometimes determine whether an optimal solution is found or not by the procedure.

At the end of the procedure, a lower bound and an upper bound have been obtained. In the next section, we discuss our computational experiments with this procedure, and we show that both bounds often coincide (and hence, are optimal).

\subsection{Computational experiments}

\subsubsection{Generation of problem instances}

We generated three sets of random instances. The first set contains 120 instances, the second set 400 instances and the third set 30 instances. Each instance falls into an instance type, characterized by the size $(\mathrm{M}, \mathrm{N})$ of the tool-job matrix and the value $\mathrm{C}$ of the capacity. Accordingly, we denote the type of an instance by a triple $(\mathrm{M}, \mathrm{N}, \mathrm{C})$. The first set of instances contains 12 instance types obtained by combining each of the matrix sizes $(20,15)$, $(40,30)$ or $(60,40)$ with four different capacity values $C_{1}, C_{2}, C_{3}, C_{4}$, as indicated in Table 3.1. For each size $(\mathrm{M}, \mathrm{N})$, we also define a pair (Min, $\mathrm{M}$ ax) of parameters with the following interpretation:

- Min = lower bound on the number of tools per job,

- Max = upper bound on the number of tools per job.

The second set of instances was created according to rules suggested by Tang and Denardo (1988b) in order to allow some comparison with the results of these authors. It involves four instance types, defined by the values of the parameters displayed in Table 3.2.

For each problem size $(\mathrm{M}, \mathrm{N})$ in the first (resp. second) set, 10 (resp. 100) random matrices $A$ were generated. For each $j=1, \ldots, N$, the $j$-th column 
Table 3.1 Parameters first set of instances

\begin{tabular}{||c|c|c|c|c|c|c||}
\hline $\begin{array}{c}\text { Problem size } \\
\text { M x N }\end{array}$ & $C_{1}$ & $C_{2}$ & $C_{3}$ & $C_{4}$ & Min & Max \\
\hline $20 \times 15$ & 6 & 8 & 10 & 12 & 2 & 6 \\
$40 \times 30$ & 15 & 17 & 20 & 25 & 5 & 15 \\
$60 \times 40$ & 20 & 22 & 25 & 30 & 7 & 20 \\
\hline
\end{tabular}

Table 3.2 Parameters second set of instances

\begin{tabular}{||c|c|c|c||}
\hline $\begin{array}{c}\text { Problem size } \\
\text { M } \times \text { N }\end{array}$ & $C_{1}$ & Min & Max \\
\hline $10 \times 10$ & 4 & 1 & 3 \\
$15 \times 20$ & 8 & 1 & 7 \\
$20 \times 30$ & 0 & 1 & 9 \\
$25 \times 30$ & 10 & 1 & 9 \\
\hline
\end{tabular}

of $\mathrm{A}$ was generated as follows. First, an integer $\mathrm{j}_{\mathrm{j}}$ was drawn from the uniform distribution over [Min, Max]: this number denotes the number of tools needed for job j, i.e. the number of 1 's in the j-th column of A. Next, a set $T_{j}$ of $t_{j}$ distinct integers were drawn from the uniform distribution over $[1, M]$ : these integers denote the tools required by job j, i.e. $a_{k j}=1$ if and only if $k$ is in $T_{j}$. Finally, we checked whether $T_{j} \subseteq T_{i}$ or $T_{i} \subseteq T_{j}$ held for any $\mathrm{i}<\mathrm{j}$. If any of these inclusions was found to hold, then the previous choice of $T_{j}$ was cancelled, and a new set $T_{j}$ was generated (as observed by Tang and Denardo (1988b) the job grouping problem trivially simplifies by removal of the columns included in other columns of the tooljob matrix). A problem instance of type $(\mathrm{M}, \mathrm{N}, \mathrm{C})$ is now obtained by combining an $\mathrm{MxN}$ tool-job matrix $\mathrm{A}$ with one of the corresponding capacities displayed in Tables 1 and 2.

The random instances described above are similar to those generated e.g. by Rajagopalan (1985), Tang and Denardo (1988b), Hwang and Shogan (1989) and Kuhn (1990). It turns out that, for these instances, the feasible groups of jobs are usually rather small (typically, 2 to 5 jobs). This can be explained by the fact that the tool requirements of the jobs are completely independent of each other, and that large subsets of jobs are therefore unlikely to be compatible. This lack of interdependence between jobs is, however, unlikely to reflect the structure of "realistic" tool-job matrices. 
Indeed, real-world instances are more likely to exhibit subsets of "similar" jobs, characterized by "similar" tool requirements. Our third set of random instances results from an attempt to capture this type of features. The parameters for this set are displayed in Table 3.3.

Table 3.3 Parameters third set of instances

\begin{tabular}{|c|c|c|c|c|c|}
\hline $\begin{array}{c}\text { Prublem size } \\
\text { M } \times \text { N }\end{array}$ & $C$ & Min & Max & Minjob & Maxjob \\
\hline $40 \times 40$ & 20 & 7 & 10 & 5 & 8 \\
$50 \times 50$ & 25 & 8 & 12 & 6 & 10 \\
$60 \times 60$ & 30 & 10 & 15 & 8 & 12 \\
\hline
\end{tabular}

Ten instances of each type $(M, N, C)$ were generated as follows. First, a number $N_{1}$ is drawn uniformly between Minjob and Maxjob, and a subset of tools $M_{1}$ of size exactly $C$ is randomly chosen. Then, we create $N_{1}$ "similar" jobs, by making sure that these jobs use only the tools in $\mathbf{M}_{1}$ (and hence, form a feasible group). These jobs are generated as explained before for the first and the second sets of instances (except that they are restricted to the tools in $M_{1}$ ). When $N_{1}$ jobs have been defined, then the procedure is iterated to produce $\mathrm{N}_{2}, \mathrm{~N}_{3}, \ldots$ additional jobs. This process stops after $\mathrm{k}$ iterations, when almost all columns of the incidence matrix have been generated (specifically, when $\sum_{i=1}^{k} N_{i} \geq N$ - Maxjob). Then, the last columns are filled independently of each other, as for the first two sets of instances.

This completes the description of our problem instances. It will be observed in the next section that the degree of difficulty of these instances is somewhat related to the relative size of the capacity with respect to the maximum number of tools used by the jobs (viz. the parameter Max). We call sparse those problem instances for which Max/C is small, and dense those for which the ratio is close to 1 . Notice, in particular, that all instances of type $\left(\mathrm{M}, \mathrm{N}, \mathrm{C}_{1}\right)$ are dense, and that the instances of type $\left(\mathrm{M}, \mathrm{N}, \mathrm{C}_{4}\right)$, as well as the instances in the third set, are rather sparse.

\subsubsection{Computational results}

The column generation procedure has been implemented as described in Section 3.4, using Turbo Pascal, and tested on the instances described in Section 3.5.1. The experiments were run on an AT personal computer with 
$16 \mathrm{MHz} 80386 \mathrm{sx}$ processor and 80387 mathematical coprocessor. No systematic attempts have been made to optimize the running times of the codes, because our primary goal was to establish the quality of the bounds computed.

Before going into detailed comments, we mention what we see as our two most interesting results. First, for all instances tested, the gap between the LP-relaxation of the set covering formulation and the value of the optimal solution was smaller than 1. In other words the column generation procedure always provided a lower bound $\mathrm{LB}_{\mathrm{CG}}$ equal to the optimal value of the job grouping problem (note, however, that this empirical observation is definitely not a theorem: indeed, it follows from Theorem 3.1 that $\mathrm{LB}_{\mathrm{CG}}$ can, for some possibly contrived examples, be arbitrarily far from the optimal value). Second, using the column generation procedure described in Section 3.4.1 we were able to solve 541 of our 550 instances to optimality. Moreover, all instances have been solved to optimality by variants of the same procedure, characterized by different choices of the parameters number of new columns generated in each iteration, value of the reduced costs under which columns are deleted, etc.).

The quality of the lower bounds $\mathrm{LB}_{\mathrm{SW}}, \mathrm{LB}_{\mathrm{MSW}}, \mathrm{LB}_{\mathrm{CG}}$ and of the solutions obtained by the sequential heuristics in step I of the procedure is compared in Table 3.4. The first column (labelled OPT) gives the average number of groups in the optimal solution, per instance type. The next three columns bear on the lower bounds; columns 5 to 11 correspond to the upper bounds delivered by the seven sequential heuristics, and the last column (labelled Best) reports on the upper bound obtained by retaining the smallest of the previous seven ones. Each entry in columns 2 to 12 has the format $\delta(\alpha)$. In a row labelled $(\mathrm{M}, \mathrm{N}, \mathrm{C})$ and a column labelled $\mathrm{X}, \delta$ is the average difference between the lower (or upper) bound $\mathrm{X}$ and the optimal value of the instance, over all instances of type $(\mathrm{M}, \mathrm{N}, \mathrm{C})$; that is, $\delta=\mathrm{X}$ OPT, where $\bar{X}$ is the average of the lower (or upper) bound $\mathrm{X}$. In columns 5 to 11 (that is, for the sequential heuristics), the entry $\alpha$ denotes the number of instances of type $(\mathrm{M}, \mathrm{N}, \mathrm{C})$ for which the upper bound $\mathrm{X}$ is best among the sequential heuristics. In the remaining columns $2,3,4$ and 12 , $\alpha$ is the number of instances for which $\mathrm{X}$ is equal to the optimal value of the instance.

As mentioned before, $\mathrm{LB}_{\mathrm{CG}}$ is equal to the optimal value for all instances tested. The lower bounds $\mathrm{LB}_{S W}$ and $\mathrm{LB}_{\mathrm{MSW}}$ are often not sufficiently sharp to prove optimality. For only $35 \%$ of the instances (especially smaller, denser instances), $\mathrm{LB}_{\mathrm{SW}}$ gives an optimal lower bound. For an 
Table 3.4 Quality of lower and upper bounds

\begin{tabular}{|c|c|c|c|c|c|c|c|c|c|c|c|c|c|}
\hline \multirow{2}{*}{\multicolumn{2}{|c|}{$\begin{array}{l}\text { Instance type } \\
\mathrm{M} \times \mathrm{N}\end{array}$}} & \multirow{2}{*}{$\begin{array}{c}\text { OPT } \\
1 \\
\end{array}$} & \multicolumn{3}{|c|}{ Lower bounds } & \multicolumn{8}{|c|}{ Upper bounds } \\
\hline & & & \multirow{2}{*}{$\begin{array}{c}\mathrm{LB}_{\mathrm{SW}} \\
2 \\
-0.5(5)\end{array}$} & \multirow{2}{*}{$\begin{array}{c}B_{\text {MSW }} \\
3 \\
-0.4(6)\end{array}$} & \multirow{2}{*}{$\begin{array}{c}\mathrm{LB}_{\mathrm{CG}} \\
4 \\
0(10)\end{array}$} & \multirow{2}{*}{$\begin{array}{c}\text { MIMU } \\
5 \\
0.3(10)\end{array}$} & \multirow{2}{*}{$\begin{array}{c}\text { MI } \\
6 \\
0.3(10)\end{array}$} & \multirow{2}{*}{$\begin{array}{c}\text { MU } \\
7 \\
0.4(9)\end{array}$} & \multirow{2}{*}{$\begin{array}{c}\begin{array}{c}\text { Whitney } \\
\text { and Gaul } \\
8 \\
0.3(10)\end{array}\end{array}$} & \multirow{2}{*}{$\begin{array}{c}\begin{array}{c}\text { Rajago- } \\
\text { palan } \\
9\end{array} \\
1.1(3)\end{array}$} & \multirow{2}{*}{$\begin{array}{r}\begin{array}{r}\text { Modified } \\
\text { Rajago- } \\
\text { palan 10 }\end{array} \\
0.3(10)\end{array}$} & \multirow{2}{*}{$\begin{array}{c}\text { Marginal } \\
\text { gain } \\
11\end{array}$} & \multirow{2}{*}{$\begin{array}{c}\text { Best } \\
12 \\
0.3(7)\end{array}$} \\
\hline $20 \times 15$ & 6 & 9.3 & & & & & & & & & & & \\
\hline & 8 & 5.7 & $-1.2(1)$ & $-1.2(1)$ & $0(10)$ & $0.4(7)$ & $0.2(9)$ & $0.7(4)$ & $0.5(6)$ & $0.8(4)$ & $0.1(10)$ & $0.6(5)$ & $0.1(9)$ \\
\hline & 10 & 3.9 & $-1.9(0)$ & $-1.8(0)$ & $0(10)$ & $0.4(7)$ & $0.4(7)$ & $0.6(5)$ & $0.4(7)$ & $1.0(2)$ & $0.4(7)$ & $0.6(5)$ & $0.1(9)$ \\
\hline & 12 & 2.9 & $-0.9(1)$ & $-0.9(1)$ & $0(10)$ & $0.2(9)$ & $0.3(8)$ & $0.6(5)$ & $0.3(8)$ & $0.9(3)$ & $0.3(8)$ & $0.5(6)$ & $0.1(9)$ \\
\hline \multirow[t]{4}{*}{$40 \times 30$} & 15 & 19.0 & $-0.7(5)$ & $-0.3(7)$ & $0(10)$ & $0.3(7)$ & $0.0(10)$ & $0.6(5)$ & $0.2(8)$ & $0.8(3)$ & $0.0(10)$ & $0.3(7)$ & $0.0(10)$ \\
\hline & 17 & 15.5 & $-0.7(5)$ & $-0.7(5)$ & $0(10)$ & $0.3(8)$ & $0.2(9)$ & $0.6(5)$ & $0.2(9)$ & $1.1(1)$ & $0.1(10)$ & $0.6(5)$ & $0.1(9)$ \\
\hline & 20 & 10.9 & $-2.1(0)$ & $-2.0(1)$ & $0(10)$ & $0.9(7)$ & $0.6(10)$ & $1.6(2)$ & $1.0(6)$ & $2.4(0)$ & $0.6(10)$ & $1.4(3)$ & $0.6(4)$ \\
\hline & 25 & 6.7 & $-4.7(0)$ & $-4.3(0)$ & $0(10)$ & $0.9(10)$ & $0.9(10)$ & $1.9(0)$ & $1.0(9)$ & $3.0(0)$ & $0.9(10)$ & $2.0(1)$ & $0.9(1)$ \\
\hline \multirow[t]{4}{*}{$60 \times 40$} & 20 & 25.9 & $-0.8(5)$ & $-0.6(7)$ & $0(10)$ & $0.5(8)$ & $0.3(10)$ & $0.6(7)$ & $0.4(9)$ & $0.9(4)$ & $0.3(10)$ & $0.6(7)$ & $0.3(7)$ \\
\hline & 22 & 22.3 & $-8.6(2)$ & $-8.6(2)$ & $0(10)$ & $0.6(5)$ & $0.1(10)$ & $1.0(3)$ & $0.5(6)$ & $1.4(0)$ & $0.1(10)$ & $0.7(5)$ & $0.1(9)$ \\
\hline & 25 & 17.0 & $-9.6(0)$ & $-9.6(0)$ & $0(10)$ & $1.5(3)$ & $0.8(9)$ & $2.5(0)$ & $1.6(1)$ & $2.3(0)$ & $0.7(10)$ & $1.9(0)$ & $0.7(3)$ \\
\hline & 30 & 12.0 & $-7.0(0)$ & $-6.9(0)$ & $0(10)$ & $1.3(6)$ & $0.9(10)$ & $2.8(0)$ & $1.3(6)$ & $3.9(0)$ & $1.0(9)$ & $3.2(0)$ & $0.9(1)$ \\
\hline $10 \times 10$ & 4 & 5.1 & $-0.7(38)$ & $-0.5(54)$ & $0(100)$ & $0.2(95)$ & $0.2(95)$ & $0.5(62)$ & $0.2(95)$ & $0.6(58)$ & $0.2(95)$ & $0.5(65)$ & $0.1(87)$ \\
\hline $15 \times 20$ & 8 & 9.3 & $-0.7(45)$ & $-0.5(57)$ & $0(100)$ & $0.4(88)$ & $0.4(90)$ & $0.7(65)$ & $0.5(83)$ & $1.2(31)$ & $0.4(89)$ & $0.8(59)$ & $0.3(68)$ \\
\hline $25 \times 30$ & 10 & 15.0 & $-0.7(47)$ & $-0.5(62)$ & $0(100)$ & $0.5(79)$ & $0.4(90)$ & $0.9(43)$ & $0.5(73)$ & $1.5(11)$ & $0.4(90)$ & $0.8(50)$ & $0.3(75)$ \\
\hline $20 \times 30$ & 10 & 14.0 & $-0.8(39)$ & $-0.6(45)$ & $0(100)$ & $0.5(81)$ & $0.4(92)$ & $1.3(26)$ & $0.6(73)$ & $1.7(15)$ & $0.4(91)$ & $1.1(33)$ & $0.3(66)$ \\
\hline $40 \times 40$ & 20 & 6.2 & $-4.2(0)$ & $-4.2(0)$ & $O(10)$ & $1.0(6)$ & $0.8(8)$ & $0.8(8)$ & $1.0(6)$ & $5.9(0)$ & $1.1(6)$ & $1.4(3)$ & $0.6(4)$ \\
\hline $50 \times 50$ & 25 & 6.3 & $-4.3(0)$ & $-4.3(0)$ & $0(10)$ & $1.5(2)$ & $1.1(6)$ & $1.3(6)$ & $0.9(6)$ & $10.3(0)$ & $1.8(4)$ & $3.9(1)$ & $0.5(5)$ \\
\hline $60 \times 60$ & 30 & 7.7 & $-5.7(0)$ & $-5.7(0)$ & $O(10)$ & $1.5(6)$ & $0.7(9)$ & $1.5(4)$ & $1.6(4)$ & $12.2(0)$ & $1.1(6)$ & $3.6(3)$ & $0.6(5)$ \\
\hline
\end{tabular}


additional $10 \%$ of the instances, $\mathrm{LB}_{\mathrm{MSW}}$ is optimal. But even $\mathrm{LB}_{\mathrm{MSW}}$ is only optimal for 2 out of the 90 sparse instances of type (M,N,C3) or (M,N,C4). This bad behaviour of $\mathrm{LB}_{\mathrm{SW}}$ and $\mathrm{LB}_{\mathrm{MSW}}$ on sparse instances is intuitively easy to understand. Indeed, as capacity increases, each pair of jobs becomes more and more likely to be compatible; hence, the sweeping procedure tends to become useless, as only small sets of pairwise incompatible jobs can be produced (notice that the same conclusion applies for the set packing lower bound $\mathrm{LB}_{\mathrm{SP}}$ - see Section 3.3.2). Tang and Denardo (1988b) recognized this weakness of the sweeping procedure, and proposed the lower bound $\mathrm{M} / \mathrm{C}$ with the hope to partially palliate it. But the latter bound is usually weak too.

As far as the sequential heuristics go, it appears from columns 5 to 11 that the MIMU, MI, Whitney and Gaul and Modified Rajagopalan rules outperform the other rules. In particular, the MI rule performs extremely well for all instance types, whereas the Modified Rajagopalan rule is especially well suited for the first two sets of instances, but is slightly weaker for the third set. In some instances, the Whitney and Gaul or the MIMU rule provide an optimal solution where the other procedures leave a gap. The MU rule is not very effective for the first two sets of instances (which may help explain why MI performs better than MIMU), but is better for the third set (it is intuitively easy to understand that, for the instances in the latter set, the Minimal Union rule tends to preserve the feasible groups which have been artificially built into the instance). The performance of the Marginal gain and the Rajagopalan rule is very weak, especially for large, sparse instances.

The best upper bound (column 12) is optimal or within one unit of optimality for nearly all instances, which explains that the average deviation from the optimal value is smaller than 1 for all instance types. For large, sparse instances, a gap often remains. Notice however that the "structured" instances in the third set (though very sparse) behave better with this respect than other sparse instances of type $(40,30,25)$ or $(60,40$,30 ). One may easily admit the idea that, for the latter instances, the built-in structure helps in finding an optimal solution (see also the comments on Table 3.5 hereunder).

Table 3.5 summarizes the results obtained by the complete procedure described in Section 3.4. We concentrate on the moments at which optimality is established; that is, Table 3.5 gives, for each instance type, the number of instances solved in each step of the procedure (the numbers in brackets refer to 4 instances for which LB $_{C G}$ could not be computed 
Table 3.5 Performance of different steps column generation procedure

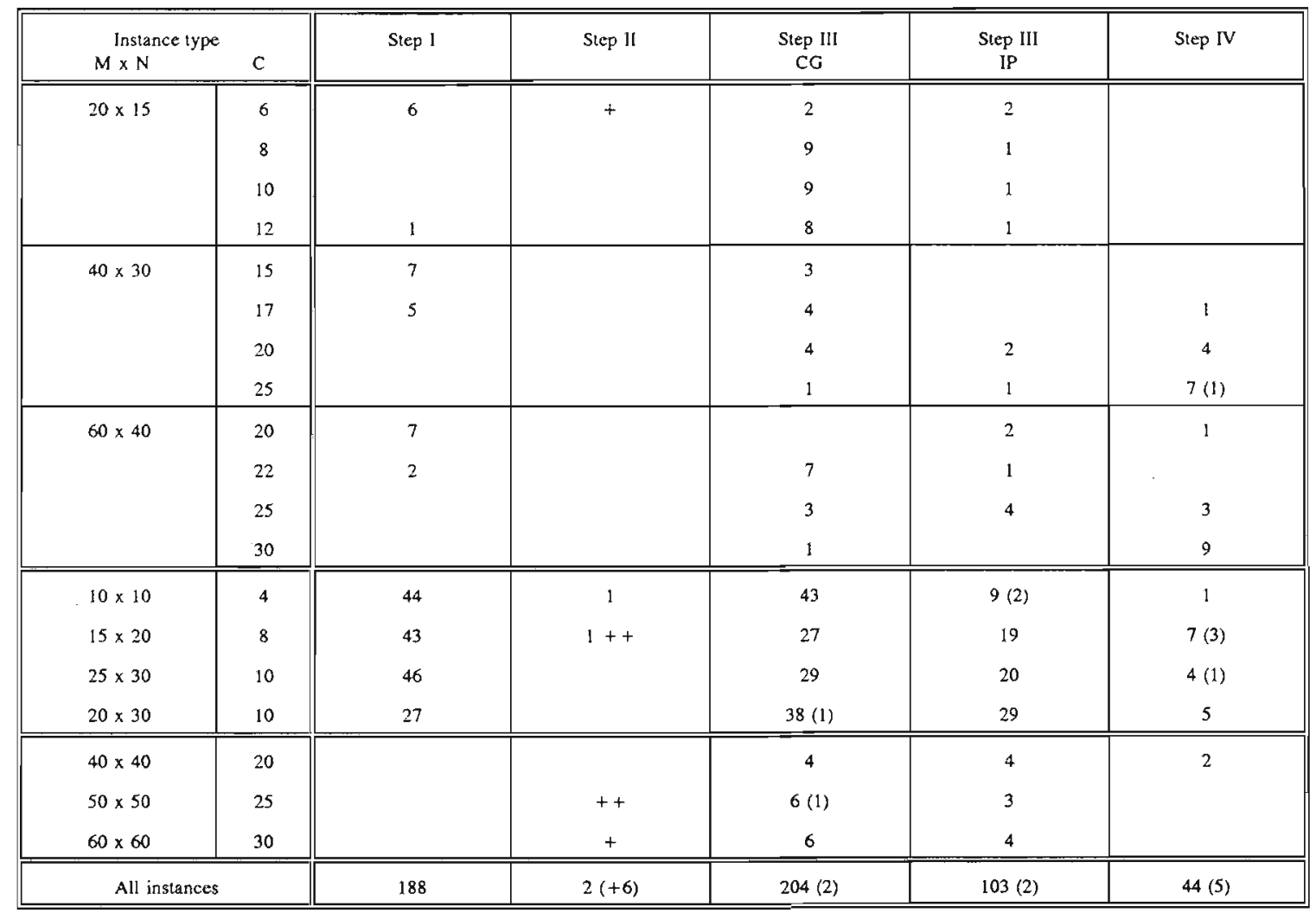


exactly because cycling occurred in Step III, and to 5 instances for which no optimal solution had been found by the heuristics after completion of Step IV - see Section 3.4; all these instances were ultimately solved to optimality by a variant of the procedure using different parameter settings). Zero values are omitted from the table to improve readability.

Thus, for instance, the column labelled "Step I" displays the number of instances for which optimality is achieved in Step I of the procedure : these are the instances for which the lower bound $\mathrm{LB}_{\mathrm{MSW}}$ is equal to Best, viz. the best sequential heuristic value. The instances for which LB $_{M S W}$ is optimal and Step II produces an optimal solution are recorded in column "Step II" (a "+" in this column denotes an instance where LB MSW is not optimal, but Step II produces an optimal solution). If optimality is not established in either Step I or Step II, the column generation process starts. Column "Step III-CG" records the number of instances for which the column generation procedure provides a lower bound $\left(\mathrm{LB}_{\mathrm{Farley}}\right.$ or $\left.\mathrm{LB}_{\mathrm{CG}}\right)$ equal to the best upper bound obtained in Steps I and II. Those instances for which an optimal 0-1 solution is found in the course of solving the set covering LP-relaxation are accounted for in column "Step III-IP". After Step III, instances remain for which the lower bound $\mathrm{LB}_{\mathrm{CG}}$ is smaller than the best available upper bound. Column "Step IV" shows the number of instances for which solving the set covering formulation with a limited number of integer variables was enough to produce an optimal solution with value equal to $\mathrm{LB}_{\mathrm{CG}}$.

For 188 out of 550 instances ( $34 \%$ ), optimality is achieved in Step I. All these are dense instances (of type $\left(\mathrm{M}, \mathrm{N}, \mathrm{C}_{1}\right)$ or $\left(\mathrm{M}, \mathrm{N}, \mathrm{C}_{2}\right)$ ), with the exception of one small $(20,15,12)$ instance. This comes as no surprise : as discussed before, both the lower bound $\mathrm{LB}_{\mathrm{MSW}}$ and the best upper bound tend to deteriorate when sparsity increases (see Table 3.4).

The upper bound computed in Step II is used to prove optimality for 8 instances only. Thus, this step does not seem very useful as far as finding good solutions goes. One should however bear in mind that the additional columns produced in this step may improve the quality of the initial set covering model, and thus reduce the number of subsequent column generation steps. More on this topic below.

Optimality is achieved in Step III for 307 instances (56\%). For 204 (37\%) of these, an optimal solution had already been found in either Step I or Step II, and only the lower bound is improved here; for the other 103 instances $(19 \%)$, both the optimal lower bound and the optimal solution 
Table 3.6 Computation times and size of problems

\begin{tabular}{|c|c|c|c|c|c|c|c|}
\hline \multicolumn{2}{|c|}{ Instance lype } & \multicolumn{3}{|c|}{ Computation times (in seconds) } & \multirow[b]{2}{*}{$\begin{array}{c}\# \text { iterations } \\
\text { col. gen. instances } \\
4\end{array}$} & \multirow[b]{2}{*}{$\begin{array}{l}\text { \# columns } \\
\text { col. gen. instances } \\
5\end{array}$} & \multirow[b]{2}{*}{$\begin{array}{l}\text { maximal feasible } \\
\text { columns, all instances } \\
6\end{array}$} \\
\hline$M \times N$ & $\mathrm{C}$ & $\begin{array}{c}\text { Heuristics } \\
\text { all instances } \\
1\end{array}$ & $\begin{array}{l}\text { Steps I-IV } \\
\text { all instances } \\
2\end{array}$ & $\begin{array}{c}\text { Steps [-IV } \\
\text { col. gen. instances } \\
3\end{array}$ & & & \\
\hline \multirow[t]{4}{*}{$20 \times 15$} & 6 & $2.6(2.5,2.8)$ & $12(2.5,44)$ & $26(15,44)$ & $4.0(2.7)$ & $23(20,29)$ & $33(18,61)$ \\
\hline & 8 & $2.3(2.3 .2 .4)$ & $26(10,40)$ & $26(10.40)$ & $3.5(1,6)$ & $29(23,41)$ & $89(51,138)$ \\
\hline & 10 & $2.2(2.1,2.4)$ & $27(12,36)$ & $27(12,36)$ & $3.0(1,5)$ & $41(19,77)$ & $189(116.284)$ \\
\hline & 12 & $2.1(2.0,2.3)$ & $30(2,51)$ & $33(14,51)$ & $3.7(1.8)$ & $43(23,74)$ & $327(238,514)$ \\
\hline \multirow[t]{4}{*}{$40 \times 30$} & 15 & $17(16,18)$ & $34(16,79)$ & $75(66,79)$ & $3.0(2,4)$ & $49(47,52)$ & $147(57,283)$ \\
\hline & 17 & $16(15,17)$ & $56(15.144)$ & $96(79,144)$ & $3.2(2.6)$ & $7 !(56,108)$ & $310(141,549)$ \\
\hline & 20 & $15(14,15)$ & $230(134,349)$ & $230(134,349)$ & $5.6(3,7)$ & $132(67,180)$ & $931(342,1640)$ \\
\hline & 25 & $14(13,14)$ & $777(422,1654)$ & $777(422,1654)$ & $8.7(7,11)$ & $252(175,344)$ & $5909(2032,8745)$ \\
\hline \multirow[t]{4}{*}{$60 \times 40$} & 20 & $42(42,43)$ & $102(42,249)$ & $242(237,249)$ & $5.7(5,7)$ & $85(63,103)$ & $215(137,311)$ \\
\hline & 22 & $39(37,42)$ & $192(41,318)$ & $229(183,318)$ & $3.5(1.6)$ & $90(62,125)$ & $404(247,694)$ \\
\hline & 25 & $37(36,38)$ & $449(263,683)$ & $449(263,683)$ & $5.5(2,7)$ & $178(121,263)$ & $1010(597,1694)$ \\
\hline & 30 & $36(35,36)$ & $1168(860,1512)$ & $1168(860,1512)$ & $7.8(7,10)$ & $288(224,364)$ & $5036(2871,9099)$ \\
\hline $10 \times 10$ & 4 & $1.1(1.0 .1 .2)$ & $7.1(1.0,35)$ & $12(6,35)$ & $2.1(1,6)$ & $13(9,24)$ & $18(7,39)$ \\
\hline $15 \times 20$ & 8 & $3.8(3.5,4.2)$ & $24(3.6,113)$ & $39(14,113)$ & $4.0(1,8)$ & $32(18,62)$ & $76(28,202)$ \\
\hline $25 \times 30$ & 10 & $12(11,13)$ & $54(11,197)$ & $90(50,197)$ & $4.3(2,11)$ & $67(36,131)$ & $263(59,879)$ \\
\hline $20 \times 30$ & 10 & $10(9.3,11)$ & $62(9.6,158)$ & $81(38,158)$ & $4.7(1,13)$ & $58(35,120)$ & $230(78,667)$ \\
\hline $40 \times 40$ & 20 & $25(24,25)$ & $1342(494,2409)$ & $1342(494,2409)$ & $14.5(6.26)$ & $184(133,213)$ & $5663(4304,6950)$ \\
\hline $50 \times 50$ & 25 & $47(46,47)$ & $2202(753,3887)$ & $2202(753,3887)$ & $24.7(6,42)$ & $247(186,325)$ & $22562(13823,32498)$ \\
\hline $60 \times 60$ & 30 & $79(78,80)$ & $4759(1967,8626)$ & $4759(1967,8626)$ & $29.9(15,49)$ & $284(172,351)$ & $31878(20336,43827)$ \\
\hline
\end{tabular}


are improved in Step III. These figures sustain our previous claims concerning the strength of the linear relaxation of the set covering formulation of the job grouping problem, and the usefulness of Step III in solving this problem to optimality (especially sparse instances).

Finally, for 44 instances (9\%), Step IV has to be performed in order to find an optimal solution. This last step is mostly required for sparse instances, but is almost never needed for the "structured" instances in the third set. This confirms our earlier conjecture that most heuristics perform better on the latter instances than on completely unstructured ones.

Table 3.6 contains information about the time required to solve the various instance types; comparison of these times provides additional information on the effectiveness of the procedure. Each entry has the format "average value (minimal value, maximal value)" (the four instances for which the column generation procedure cycles have not been taken into account when computing these average or extremal values). Column 1 gives the computation time for Step I of the procedure and column 2 records the total computation time for the whole procedure (Steps I to IV) (all times are in seconds). In columns 3,4 and 5, averages and extremal values are restricted to those instances for which execution of the column generation step (Step III) was necessary. Column 3 reports the total computation time required by Steps I-IV for these instances. Column 4 gives the number of iterations of the column generation step, that is the number of calls on the linear programming package. Column 5 indicates the maximum number of columns occurring in the linear programming subproblems. The figures in this column are to be contrasted with those in Column 6 , where the number of maximal feasible groups for each instance type is recorded. As mentioned in Section 3.2.1, this number indicates the size of the complete set covering formulation of the job grouping problem. Thus, it also gives a measure of the difficulty of the instances.

A look at column 6 immediately reveals that only the sparse instances are really big. For many of the dense instances (e.g., of type $\left(\mathrm{M}, \mathrm{N}, \mathrm{C}_{1}\right)$ ), the complete column generation model could have been explicitly generated and solved by LINDO, rather than resorting to a column generation procedure. Let us remember, however, that the characteristics of the dense instances in the second set correspond to those of the instances solved by Tang and Denardo (1988b); therefore, considering such instances allows to put our computational results in perspective.

The time required by Step I of the procedure (column 1) remains very short 
in comparison with the total computing time. It exhibits a tendency to decrease as capacity increases; this may be explained by the fact that, as capacity grows larger, the number of groups built by the heuristics decreases (see Table 3.4).

As may be expected, the total computation time grows together with the problem dimension, and especially with the number of maximal feasible columns (column 6). The number of iterations of the column generation subroutine and the size of the LP subproblems grow simultaneously. For small or dense instances, the computation times remain very short. E.g., for the instances in the second set, the average computation times are between 7 and 62 seconds, and all these instances can be solved within $31 / 2$ minutes. The computation times grow by a factor of 3 when the dimension goes from $(10,10,4)$ to $(15,20,8)$, and by a factor of 2.5 from $(15,20,8)$ to $(20,30,10)$ or $(25,30,10)$. Tang and Denardo (1988b) do not report computation times, but the number of nodes enumerated by their branchand-bound procedure for the same instance types roughly grows by factors of 10 and 15 , respectively.

For larger, sparser instances, computation times become more considerable. This can be explained in part by the larger number of iterations of the column generation step, and by the increasing size of the LP subproblems. Notice that these two factors may be influenced by the choice of some of the parameters defining the procedure; in particular, generating less columns in each step would result in a larger number of iterations, but would also decrease the time spent in each iteration. In fact, it is likely that the efficiency of the procedure could be boosted by using a heuristic to solve the generation subproblem, instead of the complete enumeration approach that we used. Complete enumeration would then only be required in the last iterations of the column generation step, to check that no more columns with negative reduced cost can be found. However, as explained in Section 3.2.3, such an approach could only be efficiently implemented if an LP solver more flexible than LINDO is available.

Finally, let us mention that the time needed to execute Step II also grows sharply with increasing capacity. This time is not singled-out in Table 3.6, but represents an important chunk of the total computation time: on average, 4 seconds (resp. 52, 146, 177, 505 and 1029 seconds) for the instances of size $(20,15)$ (resp. $(40,30),(60,40),(40,40),(50,50)$ and $(60,60))$. In order to assess the contribution of Step 2 to the efficiency of the whole procedure, we ran some experiments in which we disabled this step (more exactly, we disabled the demanding second half of this step, 
which extends the initial set covering formulation - see Section 3.4). It turned out that this modified procedure was slower, on the average, than the initial one - even though it was faster for some particular instances.

\subsection{Summary and conclusions}

In this paper, various lower and upper bounds have been proposed for the job grouping problem. In particular, we showed how the optimal value of the LP-relaxation of the set covering formulation of the problem can be computed by a column generation procedure. Although the column generation subproblem is $N P$-hard, the procedure that we implemented could solve to optimality 550 instances of the problem. Many of these instances are larger and sparser than the ones previously solved in the literature. This was only possible because of the tightness of the lower bound computed : for all 550 instances, the lower bound was equal to the optimal value of the instance.

An interesting area for further research may be the development of fast heuristics that would provide optimal results for large instances of the problem. It would also seem interesting to be able to compute good heuristic solutions and tight upper bounds for the column generation subproblem. In chapter 4 we study extensions of the present setting to situations involving multiple machines, or where each tool requires several slots in the tool magazine.

\section{Acknowledgments}

We gratefully acknowledge useful discussions on the topic of this work with Antoon W.J. Kolen and F. Soumis. 



\section{Chapter 4}

\section{The job grouping problem for flexible manufacturing systems: some extensions*}

\subsection{Introduction}

In Chapter 3 the job grouping problem for flexible manufacturing systems has been studied. This chapter concentrates on extensions of the previous model. First, the extension where tools may require several slots in the tool magazine is discussed. Next, we consider the case where several identical machines are necessary for production. In both cases, the procedures used in Chapter 3 are extended to derive strong lower and upper bounds on the optimal value of the problem and results of computational experiments are presented. In Section 4.4 we discuss the possibility to incorporate due dates in the model. Section 4.5 summarizes and concludes the chapter.

\subsection{Multiple slots}

\subsubsection{The job grouping problem}

In Chapter 3 a job grouping model is considered in which each tool requires exactly one slot in the tool magazine. However, tools often require several slots in the magazine as observed by Stecke $(1983 ; 1989)$, Kusiak (1985a), Rajagopalan $(1985 ; 1986)$ and Hwang (1986). Therefore, we relax the one-slot assumption, by allowing the number of slots necessary for a

\footnotetext{
* An article based on parts of this chapter is submitted for publication.
} 
tool to be tool-dependent. We will perform computational experiments on problems for which tools need 1 to 3 slots, as suggested by Rajagopalan (1985), Shanker and Tzen (1985) and Mazzola, Neebe and Dunn (1989).

First, we briefly discuss the set covering formulation of the job grouping problem and the column generation procedure used to solve it. The changes that are necessary in case tools need several slots in the tool magazine are incorporated in this explanation.

Assume there are $\mathrm{N}$ jobs and $\mathrm{M}$ tools. We denote by $\mathrm{s}_{\mathrm{k}}$ the number of slots that are necessary to place tool $\mathrm{k}$ in the tool magazine. The tool requirements are represented by a so-called tool-job matrix $\mathrm{A}$ of dimension $\mathrm{M} \times \mathrm{N}$, with:

$$
\begin{aligned}
a_{\mathrm{ki}} & =1 \text { if job i requires tool } \mathrm{k} \\
& =0 \text { otherwise, }
\end{aligned}
$$

for $\mathrm{k}=1, \ldots, \mathrm{M}$ and $\mathrm{i}=1, \ldots, \mathrm{N}$.

A subset (group) $S$ of jobs (or of columns of $\mathrm{A}$ ) is called feasible if the tools that are needed for these jobs together require at most $\mathrm{C}$ slots, i.e. if $\Sigma_{\mathrm{k}}\left\{\mathrm{s}_{\mathrm{k}}: \mathrm{a}_{\mathrm{ki}}=1\right.$ for some $\left.\mathrm{i} \in \mathrm{S}\right\} \leq \mathrm{C}$. We do not consider the possibility of tool overlap (where the total number of slots needed by a set of tools is strictly less than the sum of the slot requirements of the separate tools (Stecke, 1983)).

The job grouping problem consists in finding a minimum set of feasible groups such that each job is contained in (at least) one group. It can be formulated as a set covering problem, as shown in Chapter 3. Let us suppose that there exist $\mathrm{P}$ feasible groups, and let

$\mathrm{q}_{\mathrm{ij}}=1$ if job $\mathrm{i}$ is contained in the feasible group $\mathrm{j}$, $=0$ otherwise, for $\mathrm{i}=1, \ldots, \mathrm{N}$ and $\mathrm{j}=1, \ldots, \mathrm{P}$.

The job grouping problem is:

$$
\begin{aligned}
& \text { minimize } \quad \sum_{j=1}^{p} y_{j} \\
& \text { subject to } \quad \sum_{j=1}^{\mathrm{P}} \mathrm{q}_{\mathrm{ij}} \mathrm{y}_{\mathrm{j}} \geq 1 \quad \mathrm{i}=1, \ldots, \mathrm{N} \\
& y_{j} \geq 0 \quad j=1, \ldots, P \\
& y_{j} \text { integer } \quad j=1, \ldots, P
\end{aligned}
$$


where $y_{j}=1$ if group $j$ is part of the optimal covering. In comparison with the model described in Chapter 3 the introduction of tool-dependent slot requirements has influenced the set of feasible columns $\left\{q_{j}\right\}$ (where $q_{j}=$ $\left.\left(q_{1 j}, \ldots, q_{N j}\right)^{T}\right)$, but model $(4.1)$ - (4.4) remains otherwise the same.

Notice that the job grouping problem with $s_{k}>1$ for some $k$ could also be transformed into an equivalent job grouping problem with $s_{k}^{\prime}=1$ for all $k$ in a straightforward way. Namely, consider the tool job matrix $A$ and the values $s_{k}$ for each tool $k$. Now, construct a new tool job matrix $A^{\prime}$ where each row $\mathrm{k}$ in $\mathrm{A}$ is replaced by $s_{\mathrm{k}}$ similar rows in $\mathrm{A}^{\prime}$. The tool job matrix $A^{\prime}$ has $\sum_{k=1}^{M} s_{k}$ rows and $N$ columns. Solving the job grouping problem with tool job matrix $A^{\prime}, s_{k}^{\prime}=1$ for all $k$ and tool magazine capacity $C$ is equivalent to solving the job grouping problem described by the parameters $\mathrm{A}, \mathrm{s}_{\mathrm{k}}$ and $\mathrm{C}$.

This transformation has the disadvantage that it expands the size of the problem, but clearly shows that the job grouping problem where tools need several slots is a special case of the single-slot problem. Thus, the lower and upper bounding procedures developed in Chapter 3 can be easily adjusted to this case. In fact, this section can be seen as restating the procedures described in Chapter 3 in such a way that they can be applied directly to the instance $A, s_{k}, C$. The result will be a general procedure for the "multiple slots" problem with no preprocessing of the data. The new formulation will be more compact. Notice that we may expect instances with $s_{k}>1$ to have a different behaviour than the single-slot ones. This will be investigated by performing computational experiments.

\subsubsection{Lower bounds via column generation}

To find a lower bound for the set covering problem (4.1) - (4.4), we want to solve its LP-relaxation, i.e. the problem (4.1) - (4.3). A column generation procedure is used to calculate this bound, as in Chapter 3. At each iteration of the column generation procedure, we solve the LP obtained by restricting (4.1) - (4.3) to some subset $T$ of columns, i.e. we solve a problem of the form:

$$
\begin{array}{lll}
\text { minimize } & \sum_{j \in T} y_{j} & \\
\text { subject to } & \sum_{j \in T} q_{i j} y_{j} \geq 1 & i=1, \ldots, N \\
& y_{j} \geq 0 & j \in T
\end{array}
$$


for some $\mathrm{T} \subseteq\{1, \ldots, \mathrm{P}\}$. Let $\mathrm{y}^{*}$ be an optimal solution to (4.5) - (4.7) an $\lambda^{*}$ be an optimal solution to the dual of (4.5) - (4.7).

In $\mathrm{r}$ ach iteration of the column generation procedure the generation subproblem has to be solved (see Section 3.2). The generation subproblem identifies columns that have negative reduced cost and may, when added to the set covering formulation, improve the optimal solution value. The generation subproblem is

given $\lambda_{1}^{*}, \ldots, \lambda_{\mathrm{N}}^{*}$, is there a feasible group $S$ such that $\sum_{\mathrm{i} \in S} \lambda_{\mathrm{i}}^{*}>1$ ? (4.8)

After introduction of different sizes for the tools the generation subproblem can be formulated as follows (see also Hirabayashi, Suzuki and Tsuchiya (1984)):

$\operatorname{maximize} \quad \sum_{i=1}^{N} \lambda_{i}^{*} x_{i}$

subject to

$$
\begin{aligned}
& a_{k i} x_{i} \leq z_{k} \\
& \mathrm{i}=1, \ldots, \mathrm{N} \text {; } \\
& \mathrm{k}=1, \ldots, \mathrm{M} \\
& \sum_{k=1}^{M} s_{k} z_{k} \leq C \\
& \mathrm{x}_{\mathrm{i}} \in\{0,1\} \\
& \mathrm{i}=1, \ldots, \mathrm{N} \\
& \mathrm{z}_{\mathrm{k}} \in\{0,1\} \\
& \mathrm{k}=1, \ldots, \mathrm{M}
\end{aligned}
$$

where

$$
\begin{aligned}
& \mathrm{x}_{\mathrm{i}}=1 \quad \text { if job } \mathrm{i} \text { is in group } \mathrm{S} \\
& =0 \text { otherwise, } \\
& \text { for } \mathrm{i}=1, \ldots, \mathrm{N} \text {, and } \\
& \mathrm{z}_{\mathrm{k}}=1 \text { if tool } \mathrm{k} \text { is required by some job in } \mathrm{S} \text {, } \\
& =0 \text { otherwise, }
\end{aligned}
$$

for $\mathrm{k}=1, \ldots, \mathrm{M}$.

Only restriction (4.11) has changed in comparison with the generation subproblem in Section 3.2.3, so as to incorporate the number of tool slots needed for each tool. The problem (4.9) - (4.13) is $N P$-hard and we solve it using the same enumeration procedure as in Section 3.2.3. The column generation procedure that is used is basically the same as described in Section 3.2.4. When the column generation procedure stops we have an optimal solution $y^{*}$ for the LP relaxation (4.1) - (4.3). Rounding up the solution value $\sum_{j \in T} y_{j}^{*}$ to the next integer gives a lower bound for the job 
grouping problem. We will refer to the bound $\left\lceil\sum_{\mathrm{j} \in \mathcal{N}} \mathrm{y}_{j}^{*}\right]$ as $\mathrm{LB}_{\mathrm{CG}}$. We will also consider the lower bound $\mathrm{LB}_{\text {Farley }}=\left\lceil\sum_{\mathrm{i}=1}^{N} \lambda_{\mathrm{i}}^{*} / \mathrm{Z}\right\rceil$, where $\mathrm{Z}$ is the optimal solution value of the generation subproblem (see also Farley (1990)).

\subsubsection{Other lower bounds}

The sweeping procedure (Tang and Denardo, 1988b) provides a lower bound for the job grouping problem when all tools need 1 slot. Tang and Denardo (1988b) did not consider the "multiple slots" problem. However, the sweeping procedure can be modified to be applicable to "multiple slots" instances. Call two jobs compatible if they form a feasible group. The sweeping procedure sequentially creates a number of groups as follows. In each step of the procedure, a job (seed) first is selected which is compatible with the fewest number of other (not yet selected) jobs (in case of a tie, the job for which the tools necessary for the set of compatible jobs require the smallest number of slots in the tool magazine is selected). Next, the seed along with all jobs which are compatible with it are selected to form one group. The procedure is repeated until all jobs have been selected. The number of groups so created, say $\mathrm{L}$, is a valid lower bound for the job grouping problem.

We also use the trivial lower bound $\left\lceil\sum_{\mathrm{k}=1}^{\mathrm{M}} \mathrm{s}_{\mathrm{k}} / \mathrm{C}\right\rceil$. Combining this bound with $\mathrm{L}$ yields the lower bound $\mathrm{LB}_{\mathrm{SW}}=\max \left\{\left\lceil\sum_{\mathrm{k}=1}^{\mathrm{M}} \mathrm{s}_{\mathrm{k}} / \mathrm{C}\right\rceil, \mathrm{L}\right\}$.

A better lower bound can be obtained in each step of the sweeping procedure by summing the number of groups already created by the sweeping procedure and the lower bound $\left.\Gamma_{\mathrm{k}} \in \mathrm{U}_{\mathrm{i} \in \mathrm{I}} \mathrm{T}_{\mathrm{i}}{ }^{s_{\mathrm{k}}} / \mathrm{C}\right\rceil$, where $I$ is the set of "not yet selected" jobs, and $T_{i}$ is the set of tools needed for job $\mathrm{i}$. This procedure generates a sequence of valid lower bounds, the first of which is equal to $\left\lceil\sum_{k=1}^{M} s_{k} / C\right\rceil$ and the last of which is equal to $L$. We refer to this procedure as the "modified sweeping procedure". It yields a new lower bound $\mathrm{LB}_{\mathrm{MSW}}$, equal to the maximum of the bounds in the sequence.

\subsubsection{Upper bounds}

We apply sequential heuristic procedures that use a two-step approach for building groups. In the first step, a job is picked as a seed. Unless explained otherwise, we always pick a job for which the tools require the highest number of slots. Then a selection rule is used to add jobs to the group until the tool magazine capacity constraint prohibits the addition of any other job to this group. The two-step procedure is repeated until all 
jobs are assigned to some group. For selecting the next job to be assigned to a group (in step 2) a number of different rules have been considered. For a group $S$ and a job $\mathrm{i} \notin S$, let

$t_{i}=$ number of slots necessary for the tools required by job $i$;

$b_{i}=$ number of slots necessary for the tools required both by job $i$ and by some job already in $S$.

1. MIMU rule: select a job $\mathrm{i}$ for which $\mathrm{b}_{\mathrm{j}}$ is maximal; in case of a tie select a job for which $t_{i}$ is minimal (this is a straightforward generalization of the procedure by Tang and Denardo (1988b)).

2. MI rule: select a job $i$ for which $\mathrm{b}_{\mathrm{i}}$ is maximal.

3. MU rule: select a job $\mathrm{i}$ for which $\left(\mathrm{t}_{\mathrm{i}}-\mathrm{b}_{\mathrm{j}}\right)$ is minimal.

4. Whitney and Gaul rule: select job $\mathrm{i}$ for which $\left(\mathrm{b}_{\mathrm{i}}+1\right) /\left(\mathrm{t}_{\mathrm{i}}+1\right)$ is maximal (Whitney and Gaul (1985) did not consider the "multiple slots" problem, but this rule is a straightforward extension of the single-slot rule).

5. Rajagopalan rule: Each tool $\mathrm{k}$ receives a weight $\mathrm{a}_{\mathrm{k}}$ equal to the number of jobs that require tool $\mathrm{k}$ among the jobs that still have to be assigned to a group. Then, the priority of job $\mathrm{i}$ is calculated by summing the weights $s_{k} \cdot a_{k}$ of the tools that must be added to the tool magazine in case job $\mathrm{i}$ is assigned to the group. The job with the largest priority is selected first. For this rule, the first job in each group (seed) is also selected according to the same criterion (see Rajagopalan (1985)).

6. Modified Rajagopalan rule: The Rajagopalan rule can be changed in the following way: the weight $a_{k}$ for each tool $k$ is defined as the number of jobs that require tool $\mathrm{k}$ among the jobs already selected in the group. The priority of a job is the sum of the weights $s_{k} \cdot a_{k}$ of the tools that are needed for that job. The job with the highest priority is selected.

7. Marginal gain rule: The addition of job i to a group usually requires that extra tools be loaded in the tool magazine. This new tool configuration may in turn allow the execution of other, not yet selected, jobs; denote by $\mathrm{p}_{\mathrm{i}}$ the number of such jobs. This rule selects a job $i$ that maximizes $p_{i}$.

Compared to what was done in Section 3.3.1, the MIMU, MI, MU and Whitney and Gaul rule have been adjusted by simply updating the definitions of parameters $t_{i}$ and $b_{i}$. Rules 5 and 6 have been changed by incorporating the number of slots in the definition, as in Rajagopalan (1985). The Marginal gain rule uses the new definition of feasibility of a group.

The set covering heuristics can also be used as described in Section 3.3.2. 


\subsubsection{Adjusting the column generation procedure}

The column generation approach can be easily adapted to the multiple slots per tool-case. The procedure that is implemented consists of four main steps. We first briefly sketch the whole procedure before commenting on each individual step (see also Section 3.4).

Step I: Use the sequential heuristics to produce a first upper bound. Compute the simple lower bounds $\mathrm{LB}_{\mathrm{SW}}$ and $\mathrm{LB}_{\mathrm{MSW}}$. If optimality is achieved then STOP

Otherwise construct an initial set covering formulation using the groups that have been generated using the heuristic procedures.

Step II: Use the greedy heuristic to solve the initial set covering formulation. If optimality is achieved then STOP.

Step III: Solve the LP-relaxation of the current formulation.

Check whether the primal solution is integral and whether its value improves the current upper bound.

Use the dual variables to formulate the generation subproblem and generate new columns with negative reduced cost.

Calculate $\mathrm{LB}_{\text {Farley. If optimality is achieved then STOP. }}$

If no columns with negative reduced cost have been found, then continue with Step IV.

Otherwise, update the set covering formulation and repeat Step III.

Step IV: Use the last set covering formulation for finding an improved heuristic solution.

The lower and upper bounding procedures of Step I have been described in Sections 4.2.2 - 4.2.4. In Step II no additional columns are generated (contrary to what was done in Section 3.4 for the single-slot case) for reasons of time-efficiency. The set covering formulation is solved using the well-known greedy heuristic (Nemhauser and Wolsey, 1988).

The LP-relaxation is solved using the package LINDO. When the generation subproblem is solved to optimality (i.e. when a complete enumeration is performed), its optimal value $\mathrm{Z}$ is used for computing the bound $\mathrm{LB}_{\text {Farley. }}$. If this lower bound is equal to the upper bound the procedure stops. If no new column has been generated (i.e. $Z=1$ and $\mathrm{LB}_{\text {Farley }}=\mathrm{LB}_{\mathrm{CG}}$, then the column generation subroutine terminates, and we continue with step IV. Otherwise, at most 200 new columns are added to the set covering formulation. Also, to limit the size of the formulation all columns with a small reduced cost are eliminated. More precisely, columns 
for which $\sum_{i=1}^{N} q_{i j} \lambda_{i}<1-\alpha$ are removed from the formulation, where $\alpha$ is an arbitrary chosen parameter $(\alpha=0.25)$. Furthermore, columns with $\sum_{i=1}^{N} \mathrm{q}_{i j} \lambda_{i}<0.85$ are removed when the number of columns exceeds 700 (an arbitrary maximum).

Step IV of the procedure is extended in the following way. We first solve the last set covering formulation by the greedy heuristic. If this is not effective, we solve a slightly modified set covering formulation with LINDO, requiring only a limited number of variables to take $0-1$ values. More precisely, the $T$ variables which assume the largest value in the continuous solution of the set covering formulation (columns for which $\sum_{i=1}^{N} q_{i j} \lambda_{i}<0.9$ are removed to limit the size of the formulation), extended by the additional constraint $\sum_{j=1}^{\mathrm{P}} \mathrm{y}_{\mathrm{j}} \geq \mathrm{LB}_{\mathrm{CG}}$, are forced to be integer. The parameter $\mathrm{T}$ is taken equal to $\mathrm{LB}_{\mathrm{CG}}+5$ if the number of columns is smaller than 50 (resp. $\mathrm{LB}_{\mathrm{CG}}+15$ if the number of columns is between 50 and 150 , and $\mathrm{LB}_{\mathrm{CG}}+25$ otherwise). Because of the small number of integer variables, the resulting mixed 0-1 problem can be solved by LINDO's branch-and-bound subroutine (see also Section 3.4). If the solution is still fractional after this step, additional variables (that still have positive fractional values) are forced to take $0-1$ values and the formulation is solved again. This procedure is repeated until an integer solution is obtained.

\subsubsection{Computational experiments}

We generated two sets of random instances. Table 4.1 contains the parameter settings for the first set. This set of instances involves four instance sizes $(\mathrm{M}, \mathrm{N})$. The capacity of the tool magazine takes one of the values $\mathrm{C}_{1}, \mathrm{C}_{2}, \mathrm{C}_{3}$. Min (resp. Max) represent the minimal (resp. maximal) number of tool slots needed by each job. For each instance type (M, N, C) two ranges of values for $s_{k}(k=1, \ldots, M)$ are considered, as shown in column labelled ' $s_{\mathrm{k}} \in$ '. We assume that tools need a small number of tool slots $\left(s_{k} \in\{1,2,3\}\right)$, as often seems to be the case in real-world systems. Rajagopalan (1985), Shanker and Tzen (1985) and Mazzola et al. (1989) perform computational experiments using these values. Stecke (1989) gives a detailed description of a system for which the tools take either 1 or 3 slots. For the first range of values, $s_{k}$ only takes values in $\{1,2,3\}$, namely $s_{k}=1$ for $k=1, \ldots,\lfloor 2 / 3 \mathrm{M}\rfloor, s_{k}=2$ for $k=\lfloor 2 / 3 \mathrm{M}\rfloor+1$, $\ldots,\lfloor 5 / 6 \mathrm{M}\rfloor$ and $s_{k}=3$ for $k=\lfloor 5 / 6 \mathrm{M}\rfloor+1, \ldots, \mathrm{M}$. For the second range, $s_{k} \in\{1,3\}$ for all $k$, with $s_{k}=1$ for $k=1, \ldots,\lfloor 2 / 3 M\rfloor$ and $s_{k}$ $=3$ for $k=\lfloor 2 / 3 \mathrm{M}\rfloor+1, \ldots, M$.

For each problem size $(\mathrm{M}, \mathrm{N}, \mathrm{C}) 10$ random matrices $\mathrm{A}$ were generated. 
Table 4.1 Parameters first set of instances

\begin{tabular}{||c|c|c|c|c|c|c|}
\hline $\begin{array}{c}\text { Problem size } \\
\text { M x N }\end{array}$ & $\mathrm{C}_{1}$ & $\mathrm{C}_{2}$ & $\mathrm{C}_{3}$ & $\mathrm{~s}_{\mathrm{k}} \in$ & $\mathrm{Min}$ & $\mathrm{Max}$ \\
\hline $10 \times 10$ & 7 & 10 & 13 & {$[1,2,3]$} & 1 & 6 \\
$10 \times 10$ & 7 & 10 & 13 & {$[1,3]$} & 1 & 6 \\
$15 \times 20$ & 13 & 15 & 18 & {$[1,2,3]$} & 1 & 12 \\
$15 \times 20$ & 13 & 15 & 18 & {$[1,3]$} & 1 & 12 \\
$25 \times 30$ & 15 & 20 & 25 & {$[1,2,3]$} & 1 & 14 \\
$25 \times 30$ & 17 & 21 & 25 & {$[1,3]$} & 1 & 16 \\
$60 \times 40$ & 30 & 40 & 45 & {$[1,2,3]$} & 1 & 29 \\
$60 \times 40$ & 33 & 40 & 45 & {$[1,3]$} & 1 & 32 \\
\hline
\end{tabular}

For each $\mathrm{j}=1, \ldots, \mathrm{N}$, the $\mathrm{j}$-th column of $\mathrm{A}$ was generated as follows. First, an integer $t_{j}$ was drawn from the uniform distribution over [Min,Ma$x]$ : this number denotes the number of tool slots available for job $\mathrm{j}$. Next, a set $T_{j}$ of distinct integers were drawn from the uniform distribution over $[1, M]$ until at most $\mathrm{t}_{\mathrm{j}}$ slots were used, i.e. until $\sum_{\mathrm{k}} \in \mathrm{T}_{\mathrm{j}} \mathrm{s}_{\mathrm{k}}>\mathrm{t}_{\mathrm{j}}-3$. These integers denote the tools required by job j, i.e. $a_{k j}=1$ if and only if $k$ is in $T_{j}$. Finally, we checked whether $T_{j} \subseteq T_{i}$ or $T_{i} \subseteq T_{j}$ held for any $i<j$. If any of these inclusions was found to hold, then the previous choice of $T_{j}$ was cancelled, and a new set $T_{j}$ was generated.

Table 4.2 Parameters second set of instances

\begin{tabular}{||c|c|c|c|c|c|c||}
\hline $\begin{array}{c}\text { Problem size } \\
M \times N\end{array}$ & $C_{1}$ & $s_{k} \in$ & Min & Max & Minjob & Maxjob \\
\hline $30 \times 30$ & 20 & {$[1,2,3]$} & 7 & 11 & 4 & 7 \\
$30 \times 30$ & 20 & {$[1,3]$} & 7 & 11 & 4 & 7 \\
$40 \times 40$ & 30 & {$[1,2,3]$} & 10 & 16 & 5 & 8 \\
$40 \times 40$ & 30 & {$[1,3]$} & 10 & 16 & 5 & 8 \\
\hline
\end{tabular}

In Table 4.2 the parameter settings are described for the second set of instances (comparable to the third set in Section 3.5.1). For each instance class (M, N, C) 10 instances were generated. This second set explicitly takes into account the interdependence between jobs. First, a number $N_{1}$ is drawn uniformly between Minjob and Maxjob, and a subset $\mathbf{M}_{1}$, containing 
tools that together require exactly $\mathrm{C}$ tool slots, is randomly chosen. Then, we create $\mathrm{N}_{1}$ "similar" jobs, by making sure that these jobs use only the tools in $M_{1}$. These jobs are generated as explained before for the first set of instances (except that they are restricted to the tools in $M_{1}$ ). When $N_{1}$ jobs have been defined, then the procedure is iterated to produce $\mathrm{N}_{2}, \mathrm{~N}_{3}$, $\ldots$ additional jobs. This process stops after $\mathrm{k}$ iterations, when almost all columns of the incidence matrix have been generated (specifically, when $\sum_{i=1}^{k} N_{i} \geq N-$ Maxjob). Then, the last columns are filled independently of each other, as for the first set of instances.

Finally, a real-world instance described in Stecke (1989) was also tested. This instance involves 10 jobs and 141 tools, with 100 tools using 1 slot and 41 tools using 3 slots.

\subsubsection{Computational results}

The column generation procedure has been implemented using Turbo Pascal, and tested on the instances described in Section 4.2.6. The experiments were run on an AT personal computer with a $16 \mathrm{MHz} 80386 \mathrm{sx}$ processor and 80387 mathematical coprocessor. This section reports on the results of our experiments.

Using the procedure of Section 4.2.5. 271 of the 280 "multiple slots" instances were solved to optimality. The gap between the value of the LPrelaxation of the set covering formulation and the value of the optimal solution was smaller than 1 for all instances solved to optimality. In other words the lower bound $\mathrm{LB}_{\mathrm{CG}}$ was optimal for these instances. For the remaining 9 instances the procedure finished with a lower and upper bound that differed by one unit. As a matter of fact, the gap between the optimal value of the LP-relaxation of the set covering formulation and the best known upper bound amounts to maximal 1.05 for these instances. For some of these instances, a branch-and-bound procedure was eventually used to show that the upper bound was optimal and there was indeed a gap between the lower bound $\mathrm{LB}_{\mathrm{CG}}$ and the optimal solution value. Nevertheless, our experiments seem to show that the lower bound obtained by using the LPrelaxation of the set covering formulation is usually very good, even though it is not optimal for all instances.

The quality of the lower bounds $\mathrm{LB}_{S W}, \mathrm{LB}_{\mathrm{MSW}}, \mathrm{LB}_{\mathrm{CG}}$ and of the solutions obtained by the sequential heuristics in step I of the procedure is compared in Table 4.3. The first column (labelled OPT) gives the average number of groups in the optimal solution for the instances of each type that were solved to optimality using the procedure of Section 4.2.5. The next 
three columns bear on the lower bounds; columns 5 to 11 correspond to the upper bounds delivered by the seven sequential heuristics, and the last column (labelled Best) reports on the upper bound obtained by retaining the smallest of the previous seven ones. Each entry in columns 2 to 12 has the format $\delta(\alpha)$. In a row labelled $(\mathrm{M}, \mathrm{N}, \mathrm{C})$ and a column labelled $\mathrm{X}, \delta$ is the average difference over all instances of type $(\mathrm{M}, \mathrm{N}, \mathrm{C})$ between the lower (or upper) bound $\mathrm{X}$ and the best lower (or upper) bound computed for this_instance in the course of the procedure; that is, $\delta=\mathrm{X}$ - BOUND, where $\bar{X}$ is the average of the lower (or upper) bound $\mathrm{X}$ and BounD is the average of the best lower (or upper) bound. In columns 5 to 11 (that is, for the sequential heuristics), the entry $\alpha$ denotes the number of instances of type (M, N, C) for which the upper bound $X$ is best among the sequential heuristics. In the remaining columns $2,3,4$ and $12, \alpha$ is the number of instances for which $\mathrm{X}$ is equal to the best lower (or upper) bound of the instance.

For 271 out of 280 instances the best lower bound $\left(\mathrm{LB}_{\mathrm{CG}}\right)$ is equal to the optimal solution value. For the remaining 9 instances the best lower bound and the best upper bound differ by one group. The lower bounds $\mathrm{LB}_{S W}$ and $\mathrm{LB}_{\mathrm{MSW}}$ are seldom sharp (only for small instances and instances of type $\left(M, N, C_{1}\right)$ ). Table 4.3 also shows that the $M I$ and the Modified Rajagopalan rules (in that order) outperform the other rules. The performance of the MIMU and the Whitney and Gaul rule is quite good. The MU and the Marginal gain rules are much weaker. The Rajagopalan rule performs even worse (especially for the instances of the second set). Taking the best solution of the sequential heuristics, a solution is obtained which is optimal or close to optimality (gap of 1) for nearly all instances (for about half of the larger instances an optimal solution is obtained). Because of the poor quality of the lower bounds $\mathrm{LB}_{S W}$ and $\mathrm{LB}_{\mathrm{MSW}}$ the column generation procedure is needed for a large majority of the instances. The instances for which a gap remained at the end of the procedure can be found in rows with $\alpha<10$ in column 4 ( $\left.\mathrm{LB}_{\mathrm{CG}}\right)$.

Table 4.4 summarizes the results obtained by the complete procedure described in Section 4.2.5. We concentrate on the moments at which optimality is established; that is, Table 4.4 gives, for each instance type, the number of instances solved in each step of the procedure (the numbers in brackets refer to 4 instances for which no optimal solution had been found after completion of Step IV; all these instances were ultimately solved to optimality by a variant of the procedure using different parameter settings). Zero values are omitted from the table to improve readability. 
Table 4.3 Quality of lower and upper bounds

\begin{tabular}{|c|c|c|c|c|c|c|c|c|c|c|c|c|c|}
\hline \multicolumn{2}{|c|}{ Instance type } & \multirow[b]{2}{*}{$\begin{array}{c}\mathrm{OPT} \\
1 \\
\end{array}$} & \multicolumn{3}{|c|}{ Lower bounds } & \multicolumn{8}{|c|}{ Upper bounds } \\
\hline $\begin{array}{l}M \times N \\
\left(s_{k} \in\right)\end{array}$ & $\mathrm{C}$ & & $\begin{array}{c}\mathrm{LB}_{S W} \\
2\end{array}$ & $\begin{array}{c}\mathrm{LB}_{\mathrm{MSW}} \\
3\end{array}$ & $\begin{array}{c}\mathrm{LB}_{\mathrm{CG}} \\
4\end{array}$ & $\begin{array}{c}\text { MIMU } \\
5\end{array}$ & $\begin{array}{l}\text { MI } \\
6\end{array}$ & $\begin{array}{c}\mathrm{MU} \\
7\end{array}$ & $\begin{array}{l}\text { Whilney } \\
\text { and Gaul } \\
8\end{array}$ & $\begin{array}{c}\text { Rajagopa- } \\
\text { lan } \\
9\end{array}$ & $\begin{array}{l}\text { Modified } \\
\text { Rajagopa- } \\
\text { lan } 10\end{array}$ & $\begin{array}{c}\text { Marginal } \\
\text { gain } \\
11\end{array}$ & $\begin{array}{c}\text { Best } \\
12\end{array}$ \\
\hline \multirow[t]{2}{*}{$10 \times 10$} & 7 & 3.3 & $-0.3(7)$ & $-0.3(7)$ & $0(10)$ & 0.169 & $0.3(7)$ & $0.1(9)$ & $0.1(9)$ & $0.5(5)$ & $0.2(8)$ & $0.6(4)$ & $0.0(10)$ \\
\hline & 10 & 2.0 & $0.0(10)$ & $0.0(10)$ & $0(10)$ & $0.2(10)$ & $0.2(10)$ & $0.4(8)$ & $0.4(8)$ & $0.5(7)$ & $0.2(10)$ & $0.4(8)$ & $0.2(8)$ \\
\hline$[1,2,3]$ & 13 & 2.0 & $0.0(10)$ & $0.0(10)$ & $0(10)$ & $0.0(10)$ & $0.0(10)$ & $0.0(10)$ & $0.0(10)$ & $0.0(10)$ & $0.0(10)$ & $0.0(10)$ & $0.0(10)$ \\
\hline \multirow[t]{2}{*}{$10 \times 10$} & 7 & 4.3 & $-0.4(6)$ & $-0.1(9)$ & $0(10)$ & $0.1(10)$ & $0.1(10)$ & $0.2(9)$ & $0.1(10)$ & $0.4(7)$ & $0.1(10)$ & $0.3(8)$ & $0.1(9)$ \\
\hline & 10 & 2.8 & $-0.8(2)$ & $-0.8(2)$ & $0(10)$ & $0.0(10)$ & $0.0(10)$ & $0.1(9)$ & $0.0(10)$ & $0.2(8)$ & $0.0(10)$ & $0.0(10)$ & $0.0(10)$ \\
\hline$[1,3]$ & 13 & 2.0 & $0.0(10)$ & $0.0(10)$ & $0(10)$ & $0.0(10)$ & $0.0(10)$ & $0.0(10)$ & $0.0(10)$ & $0.4(6)$ & $0.0(10)$ & $0.2(8)$ & $0.0(10)$ \\
\hline \multirow[t]{2}{*}{$15 \times 20$} & 13 & 6.0 & $-1.2(1)$ & $-1.0(2)$ & $0(10)$ & $0.7(6)$ & $0.3(10)$ & $1.2(1)$ & $0.8(5)$ & $1.6(1)$ & $0.3(10)$ & $0.6(7)$ & $0.3(7)$ \\
\hline & 15 & 4.2 & $-1.5(0)$ & $-1.3(0)$ & $0(10)$ & $0.5(8)$ & $0.3(10)$ & $0.8(5)$ & $0.5(8)$ & $1.6(1)$ & $0.4(9)$ & $0.8(5)$ & $0.3(7)$ \\
\hline$[1,2,3]$ & 18 & 2.8 & $-0.8(2)$ & $-0.8(2)$ & $0(10)$ & $0.1(9)$ & $0.0(10)$ & $0.2(8)$ & $0.1(9)$ & $0.9(2)$ & $0.2(8)$ & $0.4(6)$ & $0.0(10)$ \\
\hline \multirow[t]{2}{*}{$15 \times 20$} & 13 & 7.6 & $-1.0(2)$ & $-0.8(3)$ & $0(10)$ & $0.5(8)$ & $0.4(9)$ & $1.1(3)$ & $0.6(7)$ & $1.1(4)$ & $0.4(9)$ & $0.9(4)$ & $0.3(7)$ \\
\hline & 15 & 5.1 & $-1.5(1)$ & $-1.4(1)$ & $O(10)$ & $0.6(6)$ & $0.4(8)$ & $1.0(3)$ & $0.5(7)$ & $2.1(0)$ & $0.4(8)$ & $1.0(2)$ & $0.2(8)$ \\
\hline$[1,3]$ & 18 & 3.3 & $-1.3(0)$ & $-1.1(0)$ & $O(10)$ & $0.5(6)$ & $0.3(8)$ & $0.5(7)$ & $0.5(7)$ & $0.8(5)$ & $0.3(8)$ & $0.6(5)$ & $0.1(7)$ \\
\hline \multirow[t]{2}{*}{$25 \times 30$} & 15 & 13.4 & $-1.2(2)$ & $-0.8(6)$ & $0(10)$ & $0.5(8)$ & $0 . \overline{4(9)}$ & $0.9(5)$ & $0.4(9)$ & $2.3(0)$ & $0.3(10)$ & $0.8(5)$ & $0.3(7)$ \\
\hline & 20 & 7.2 & $-3.7(0)$ & $-3.3(0)$ & $O(10)$ & $0.9(5)$ & $0.5(8)$ & $1.3(3)$ & $0.8(5)$ & $3.2(0)$ & $0.9(4)$ & $1.5(2)$ & $0.3(6)$ \\
\hline$[1,2,3]$ & 25 & 4.4 & $-2.4(0)$ & $-2.4(0)$ & $0(10)$ & $0.6(9)$ & $0.5(10)$ & $1.0(5)$ & $0.9(6)$ & $2.5(0)$ & $0.7(8)$ & $1.2(4)$ & $0.5(4)$ \\
\hline \multirow[t]{2}{*}{$25 \times 30$} & 17 & 12.8 & $-0.9(3)$ & $-0.8(4)$ & $0(10)$ & $0.8(3)$ & $0.2(9)$ & $1.1(2)$ & $0.6(5)$ & $2.4(0)$ & $0.2(9)$ & $1.0(1)$ & $0.1(9)$ \\
\hline & 21 & 7.9 & $-2.8(0)$ & $-2.7(0)$ & $0(10)$ & $1.3(5)$ & $0.8(10)$ & $1.7(2)$ & $1.2(6)$ & $3.3(0)$ & 0.9 (9) & $2.0(0)$ & $0.8(2)$ \\
\hline$[1,3]$ & 25 & 5.6 & $-3.2(0)$ & $-2.9(0)$ & $0(10)$ & $0.8(6)$ & $0.7(7)$ & $1.3(1)$ & $0.9(5)$ & $3.1(0)$ & $0.7(7)$ & $1.3(2)$ & $0.4(5)$ \\
\hline \multirow[t]{2}{*}{$60 \times 40$} & 30 & 18.1 & $-11.3(0)$ & $-11.3(0)$ & $0(10)$ & $0.8(5)$ & $0.4(9)$ & $1.5(1)$ & $0.8(5)$ & $1.6(1)$ & $0.4(9)$ & $1.2(4)$ & $0.3(7)$ \\
\hline & 40 & 10.7 & $-5.1(0)$ & $-4.8(0)$ & $0(10)$ & $1.6(1)$ & $0.6(9)$ & $2.2(0)$ & $1.4(4)$ & $2.9(0)$ & $0.7(8)$ & $2.0(0)$ & $0.5(4)$ \\
\hline$[1,2,3]$ & 45 & 8.6 & $-6.0(0)$ & $-5.6(0)$ & $0(10)$ & $1.3(4)$ & $0.5(10)$ & $1.9(1)$ & $1.0(5)$ & $3.5(0)$ & $0.8(7)$ & $2.2(0)$ & $0.5(5)$ \\
\hline \multirow[t]{2}{*}{$60 \times 40$} & 33 & 19.4 & $-11.8(0)$ & $-11.8(0)$ & $0(10)$ & $0.5(9)$ & $0.4(10)$ & $1.2(3)$ & $0.5(9)$ & $1.9(1)$ & $0.4(10)$ & $1.1(4)$ & $0.4(6)$ \\
\hline & 40 & 13.7 & $-6.1(0)$ & $-6.1(0)$ & $0(10)$ & $1.6(2)$ & $0.6(9)$ & $2.1(0)$ & $1.5(2)$ & $2.9(0)$ & $0.7(8)$ & $1.9(\mathrm{i})$ & $0.5(5)$ \\
\hline$[1,3]$ & 45 & 11.1 & $-5.6(0)$ & $-5.5(0)$ & $0(10)$ & $1.3(5)$ & $0.8(10)$ & $2.4(0)$ & $1.8(1)$ & $3.3(0)$ & $1.1(7)$ & $2.4(0)$ & $0.8(2)$ \\
\hline $30 \times 30[1,2,3]$ & 20 & 5.3 & $-2.3(0)$ & $-2.3(0)$ & $0(10)$ & $0.6(7)$ & $0.6(7)$ & $0.8(5)$ & $0.6(7)$ & $3.2(0)$ & $0.5(8)$ & $1.4(1)$ & $0.3(6)$ \\
\hline $30 \times 30[1,3]$ & 20 & 5.5 & $-2.5(0)$ & $-2.5(0)$ & $O(10)$ & $0.7(8)$ & $0.9(6)$ & $1.0(5)$ & $0.7(8)$ & $3.2(0)$ & $0.7(8)$ & $1.3(3)$ & $0.5(5)$ \\
\hline $40 \times 40[1,2,3]$ & 30 & 6.4 & $-3.4(0)$ & $-3.4(0)$ & $0(10)$ & $1.3(2)$ & $0.7(7)$ & $1.5(3)$ & $1.1(3)$ & $5.0(0)$ & $1.3(2)$ & $2.3(1)$ & $0.4(5)$ \\
\hline $40 \times 40[1,3]$ & 30 & 6.8 & $-3.7(0)$ & $-3.7(0)$ & $0(10)$ & $1.2(7)$ & $0.8(9)$ & $1.4(5)$ & $1.3(5)$ & $5.0(0)$ & $1.3(5)$ & $1.9(2)$ & $0.7(3)$ \\
\hline
\end{tabular}


Table 4.4 Performance of different steps of the column generation procedure

\begin{tabular}{|c|c|c|c|c|c|c|c|c|}
\hline$M \times N\left(s_{k} \in\right)$ & $\mathrm{C}$ & Step I & Srep II & $\begin{array}{l}\text { Step III } \\
\text { CG }\end{array}$ & $\begin{array}{l}\text { Step III } \\
\text { IP }\end{array}$ & $\begin{array}{c}\text { Step IV } \\
\text { A }\end{array}$ & $\begin{array}{c}\text { Step IV } \\
\text { B }\end{array}$ & Gap \\
\hline $\begin{array}{l}10 \times 10 \\
{[1,2,3]}\end{array}$ & $\begin{array}{c}7 \\
10 \\
13\end{array}$ & $\begin{array}{c}7 \\
8 \\
10\end{array}$ & & 3 & 2 & & & \\
\hline $\begin{array}{c}10 \times 10 \\
{[1,3]}\end{array}$ & $\begin{array}{c}7 \\
10 \\
13\end{array}$ & $\begin{array}{c}8 \\
2 \\
10\end{array}$ & & $\begin{array}{l}1 \\
8\end{array}$ & 1 & & & \\
\hline $\begin{array}{l}15 \times 20 \\
{[1,2,3]}\end{array}$ & $\begin{array}{l}13 \\
15 \\
18 \\
\end{array}$ & $\begin{array}{l}1 \\
2 \\
\end{array}$ & & $\begin{array}{l}6 \\
7 \\
8 \\
\end{array}$ & $\begin{array}{l}1 \\
1\end{array}$ & $\begin{array}{l}2 \\
2\end{array}$ & & \\
\hline $\begin{array}{l}15 \times 20 \\
{[1.3]}\end{array}$ & $\begin{array}{l}13 \\
15 \\
18\end{array}$ & 2 & & $\begin{array}{l}5 \\
8 \\
7 \\
\end{array}$ & $\begin{array}{l}2 \\
2\end{array}$ & $\begin{array}{l}1 \\
1 \\
\end{array}$ & & 2 \\
\hline $\begin{array}{l}25 \times 30 \\
{[1,2,3]}\end{array}$ & $\begin{array}{l}15 \\
20 \\
25\end{array}$ & 4 & & $\begin{array}{l}3 \\
6 \\
4\end{array}$ & $\begin{array}{l}3 \\
1\end{array}$ & $\begin{array}{c}2 \\
2(3)\end{array}$ & & $\begin{array}{l}1 \\
1\end{array}$ \\
\hline $\begin{array}{c}25 \times 30 \\
{[1,3]}\end{array}$ & $\begin{array}{l}17 \\
21 \\
25\end{array}$ & 4 & + & $\begin{array}{l}6 \\
2 \\
5\end{array}$ & $\begin{array}{l}3 \\
1\end{array}$ & $\begin{array}{l}5 \\
3\end{array}$ & & 1 \\
\hline $\begin{array}{l}60 \times 40 \\
{[1,2,3]}\end{array}$ & $\begin{array}{l}30 \\
40 \\
45\end{array}$ & & & $\begin{array}{l}7 \\
4 \\
5\end{array}$ & $\begin{array}{l}2 \\
1\end{array}$ & $\begin{array}{c}1 \\
3(1) \\
3\end{array}$ & 2 & 1 \\
\hline $\begin{array}{c}60 \times 40 \\
{[1,3]}\end{array}$ & $\begin{array}{l}33 \\
40 \\
45\end{array}$ & & & $\begin{array}{l}6 \\
5 \\
2\end{array}$ & $\begin{array}{l}3 \\
2\end{array}$ & $\begin{array}{l}4 \\
1 \\
4\end{array}$ & $\begin{array}{l}1 \\
2\end{array}$ & \\
\hline $\begin{array}{c}30 \times 30[1,2,3] \\
30 \times 30[1,3] \\
40 \times 40[1,2,3] \\
40 \times 40[1,3]\end{array}$ & $\begin{array}{l}20 \\
20 \\
30 \\
30 \\
\end{array}$ & & & $\begin{array}{l}6 \\
5 \\
5 \\
3 \\
\end{array}$ & $\begin{array}{l}2 \\
3 \\
2 \\
\end{array}$ & $\begin{array}{l}1 \\
1 \\
4 \\
4 \\
\end{array}$ & 1 & $\begin{array}{l}1 \\
1 \\
1 \\
\end{array}$ \\
\hline \multicolumn{2}{|c|}{ All instances } & 58 & $0(+1)$ & 127 & 32 & $44(4)$ & 6 & 9 \\
\hline
\end{tabular}


Thus, for instance, the column labelled "Step I" displays the number of instances for which optimality is achieved in Step I of the procedure : these are the instances for which the lower bound $\mathrm{LB}_{\mathrm{MSW}}$ is equal to Best, viz. the best sequential heuristic value. The instances for which $\mathrm{LB}_{\mathrm{MSW}}$ is optimal and Step II produces an optimal solution are recorded in column "Step II" (a " + " in this column denotes an instance where LB $_{\text {MSW }}$ is not optimal, but Step II produces an optimal solution). If optimality is not established in either Step I or Step II, the column generation process starts. Column "Step III-CG" records the number of instances for which the column generation procedure provides a lower bound $\left(\mathrm{LB}_{\mathrm{Farley}}\right.$ or $\left.\mathrm{LB}_{\mathrm{CG}}\right)$ equal to the best upper bound obtained in Steps I and II. Those instances for which an optimal $0-1$ solution is found in the course of solving the set covering LP-relaxation are accounted for in column "Step III-IP". After Step III, instances remain for which the lower bound $\mathrm{LB}_{\mathrm{CG}}$ is smaller than the best available upper bound. Column "Step IV - A" shows the number of instances for which solving the set covering formulation with a limited number of integer variables was enough to produce an optimal solution with value equal to $\mathrm{LB}_{\mathrm{CG}}$. Column "Step IV - B" shows the number of instances for which Step IV - A did not suffice, but for which an optimal solution was obtained after more variables were forced to take 0-1 values, as described in Section 4.2.5. Column "Gap" displays the number of instances for which the best lower bound $\left(\mathrm{LB}_{\mathrm{CG}}\right)$ was strictly smaller than the best known upper bound at the end of the procedure.

Table 4.4 shows that for $21 \%$ of the instances optimality is achieved in Step I (mainly smaller instances). For only one instance Step II offered a better upper bound. In Step III optimality is achieved for 159 instances (57 $\%)$. For 32 instances (11\%) the upper bound was improved in Step III. For 63 instances $(23 \%)$ Step IV had to be performed. Four of these instances were solved with different parameter settings. For another 6 instances the solution of the set covering formulation remained fractional after a number of variables were forced to take 0-1 values. Nine instances could not be solved. For these instances a gap remained between $\mathrm{LB}_{\mathrm{CG}}$ and the best upper bound (the largest gap between the LP-relaxation value and the best upper bound amounts 1.05). A comparison with results of Section 3.6 shows that these instances seem to be harder than those considered in the previous study (see also column 6 in Table 4.5).

Table 4.5 contains information about the time required to solve the various instance types; comparison of these times provides additional information on the effectiveness of the procedure. Each entry has the format "average value (minimal value, maximal value)" (the four instances for which the 
Table 4.5 Computation times and size of problems

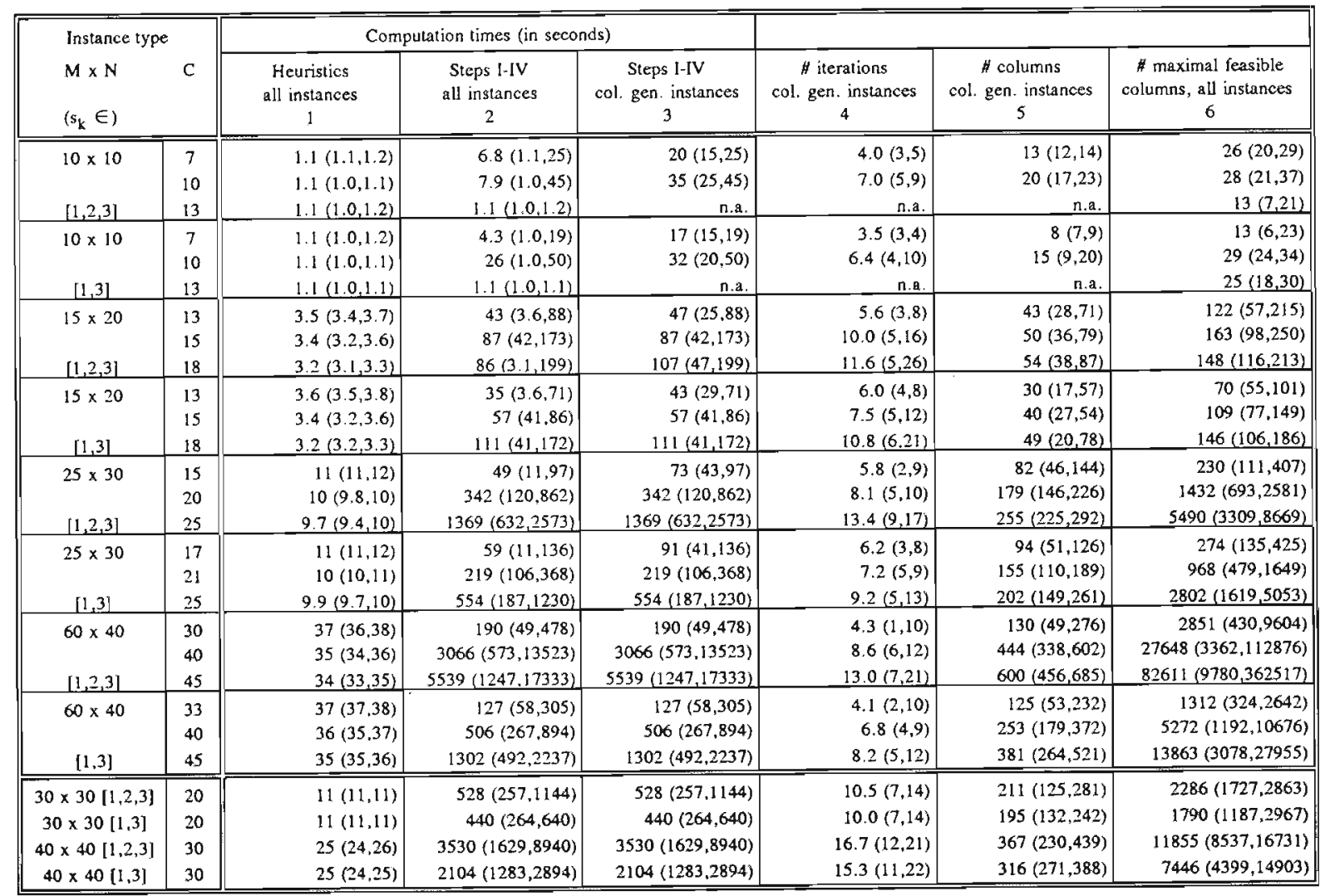


column generation procedure cycles have not been taken into account when computing these average or extremal values). Column 1 gives the computation time for Step I of the procedure and column 2 records the total computation time for the whole procedure (Steps I to IV) (all times are in seconds). In columns 3, 4 and 5, averages and extremal values are restricted to those instances for which execution of the column generation step (Step III) was necessary. Column 3 reports the total computation time required by Steps I-IV for these instances. Column 4 gives the number of iterations of the column generation step, that is the number of calls on the linear programming package. Column 5 indicates the maximum number of columns occurring in the linear programming subproblems. The figures in this column are to be contrasted with those in Column 6 , where the number of maximal feasible groups for each instance type is recorded. This number indicates the size of the complete set covering formulation of the job grouping problem (see Section 4.2.1). Thus, it also gives a measure of the difficulty of the instances.

The computation times in Table 4.5 show that the large instance (especially of type $\left(\mathrm{M}, \mathrm{N}, \mathrm{C}_{3}\right)$ ) take a lot of time to reach optimality. This is due to the many calls to LINDO and the size of the set covering formulations that have to be solved in each step. For larger instances columns 2 and 3 are similar, because all instances need the execution of the column generation procedure. Column 4 shows that at most 26 calls to LINDO are necessary. The maximal average number of columns lies around 600 (which is close to the maximum of 700 columns). The last column shows that the size of the complete set covering formulation for the large instances is indeed very large.

The real-world instance of Stecke (1989) was solved to optimality by 6 out of 7 sequential heuristics (not recorded in Tables 4.5, 4.6 and 4.7). The lower bound $\mathrm{LB}_{\mathrm{CG}}$ was optimal, in contrast with the other lower bounds.

\subsection{Multiple machines}

\subsubsection{The job grouping problem}

In practice a flexible machine is likely to be part of a system of several machines. Rajagopalan (1985) and Tang and Denardo (1988b) developed models to describe "multiple machine" problems. In this section we consider the job grouping problem for a number of identical machines, where each job has to be processed by each machine. Early flexible 
manufacturing systems consisted of different types of machines. Nowadays many machines and tools have become so versatile that only one type of CNC machine can be used to produce a wide variety of part types (Hwang and Shogan, 1989). Many FMSs are configured with a group of these general-purpose CNC machines (Jaikumar, 1986; Jaikumar and Van Wassenhove, 1989) and a job entering such a system is routed to one of the available machines. If each job has to be processed by only one machine, the single machine job grouping model (see Chapter 3) can be used, extended by a final step in which the groups are assigned to the different machines. However, other criteria like workload balancing tend to become more important for these cases. This leads to a different type of problems which we did not consider in this research.

Thus, we assume that each job has certain tool requirements on each machine. These requirements are described by the tool job "matrix" $\left(a_{\text {kim }}\right)$, where $\mathrm{a}_{\text {kim }}=1$ if tool $\mathrm{k}(\mathrm{k}=1, \ldots, \mathrm{M})$ is used for job $\mathrm{i}(\mathrm{i}=1, \ldots, \mathrm{N})$ on machine $\mathrm{m}(\mathrm{m}=1, \ldots, \mathrm{V})$ and $\mathrm{a}_{\mathrm{kim}}=0$ otherwise. We assume that each tool needs 1 slot in the tool magazine, and that all machines have the same capacity $\mathrm{C}$ (the latter assumption is mostly for ease of notation, and can be easily removed). The job grouping problem is to find a partition of the jobs into a minimum number of groups, such that the jobs in each group do not require more tools on each machine than can be stored in the tool magazine of the machine. A set covering formulation (4.1) - (4.4) of the problem is still valid. The columns in the formulation represent the groups that are feasible on all machines.

\subsubsection{Lower bounds via column generation}

A lower bound can again be computed by solving the linear relaxation of the formulation (4.1) - (4.4) using a column generation approach. However, a different generation subproblem must now be solved in order to find columns that can improve the solution value of (4.5) - (4.7). Indeed, the restrictions (4.10) and (4.11) must be included for each machine (with $s_{k}=1$ for all $k$ ), thus leading to the following formulation of the generation subproblem:

$\operatorname{maximize} \quad \sum_{i=1}^{N} \lambda_{i} x_{i}$

subject to

$$
\begin{aligned}
& \mathrm{a}_{\mathrm{kim}} \mathrm{x}_{\mathrm{i}} \leq \mathrm{z}_{\mathrm{km}} \quad \mathrm{i}=1, \ldots, \mathrm{N} \\
& \mathrm{k}=1, \ldots, \mathrm{M} \\
& \mathrm{m}=1, \ldots, \mathrm{V}
\end{aligned}
$$




$$
\begin{array}{ll}
\sum_{\mathrm{k}=1}^{\mathrm{M}} \mathrm{z}_{\mathrm{km}} \leq \mathrm{C} & \mathrm{m}=1, \ldots, \mathrm{V} \\
\mathrm{x}_{\mathrm{i}} \in\{0,1\} & \mathrm{i}=1, \ldots, \mathrm{N} \\
\mathrm{z}_{\mathrm{km}} \in\{0,1\} & \mathrm{k}=1, \ldots, \mathrm{M} ; \\
& \mathrm{m}=1, \ldots, \mathrm{V}
\end{array}
$$

where

$$
\begin{aligned}
& \mathrm{x}_{\mathrm{i}}=1 \quad \text { if job } \mathrm{i} \text { is in group } \mathrm{S} \\
& =0 \quad \text { otherwise, } \\
& \text { for } \mathrm{i}=1, \ldots, \mathrm{N} \text {, and } \\
& \begin{aligned}
\mathrm{z}_{\mathrm{km}} & =1 & & \text { if tool } \mathrm{k} \text { is required by some job in } \mathrm{S} \text { on machine } \mathrm{m}, \\
& =0 & & \text { otherwise, }
\end{aligned} \\
& \text { for } \mathrm{k}=1, \ldots, \mathrm{M} ; \mathrm{m}=1, \ldots, \mathrm{V} \text {. }
\end{aligned}
$$

As previously, we solve this subproblem by complete enumeration (see Section 3.2.3). The lower bounds $\mathrm{LB}_{\text {Farley }}$ and $\mathrm{LB}_{\mathrm{CG}}$ are defined as in Section 3.2.4.

\subsubsection{Other lower bounds}

The sweeping procedure can be adjusted as described by Tang and Denardo (1988b). The concept of compatibility is changed for the "multiple machines" case. Two jobs are compatible if they form a feasible group on all machines. A number of groups are sequentially created as follows. In each step of the procedure, first a job (seed) is selected which is compatible with the smallest number of other (not yet selected) jobs (in case of a tie the job, for which the set of compatible jobs requires the smallest number of tools on all machines is selected). Next, the seed along with all jobs which are compatible with it, are selected to form one group. The procedure is repeated until all jobs have been selected. The number of groups so created, say $\mathrm{L}$ is a valid lower bound for the job grouping problem. We also have the trivial lower bound $\lceil\mathrm{M} / \mathrm{C}\rceil$. Combining this bound with $\mathrm{L}$ yields the lower bound $\mathrm{LB}_{\mathrm{SW}}=\{\lceil\mathrm{M} / \mathrm{C}\rceil, \mathrm{L}\}$.

Another lower bound can be obtained in each step of the sweeping procedure by summing the number of groups already created by the procedure and the lower bound $\max _{\mathrm{m}}\left\{\left\lceil\left|\mathrm{U}_{\mathrm{i} \in \mathrm{I}} \mathrm{T}_{\mathrm{im}}\right| / \mathrm{C}\right\rceil\right\}$, where I is the set of "not yet selected" jobs, and $\mathrm{T}_{\text {im }}$ is the set of tools needed for job $\mathrm{i}$ on machine $\mathrm{m}$. This procedure generates a sequence of valid lower bounds, the first of which is equal to $\lceil\mathrm{M} / \mathrm{C}\rceil$ and the last of which is equal to $\mathrm{L}$. We refer to this procedure as the "modified sweeping 
procedure". It yields a new lower bound, equal to the maximum of the bounds in the sequence, which we denote by $\mathrm{LB}_{\mathrm{MSW}}$.

\subsubsection{Upper bounds}

The MIMU, MI, MU and Whitney and Gaul rules are changed by simply adjusting the definitions of the parameters $b_{i}$ and $t_{i}$ introduced in Section 3.3.1. For a group $S$ and a job i $\notin S$, let

$b_{i}=$ the sum over all machines of the number of tools required by job $i$ $t_{i}=$ the sum over all machines of the number of tools required both by job $\mathrm{i}$ and by some job already in $\mathrm{S}$.

For the MIMU rule these changes have been described by Tang and Denardo (1988b).

The Rajagopalan rule (resp. Modified Rajagopalan-rule) is changed similarly. For each machine $\mathrm{m}$, each tool $\mathrm{k}$ receives a weight $\mathrm{a}_{\mathrm{km}}$, defined as $a_{k}$ was for the one machine case. Next the priority of job $i$ is calculated by summing the weights $a_{k m}$ over all tools that must be added to the tool magazine of machine $m$ (resp. over all tools needed for job $i$ on machine $m$ ) when job $i$ is assigned to the current group, and over all machines. Rajagopalan (1985) also assigns weights to the different machines, based on the ratio of the total number of tool slots needed for the jobs executed on the machine to the capacity of the tool magazine. We decided to use equal weights for all machines. The Marginal gain rule is defined as in the single machine case. For all sequential heuristics the selection of the first job in each group is also based on the cumulative measures mentioned above.

All set covering heuristics can also be used as described in Section 3.3.2.

\subsubsection{Adjusting the column generation procedure}

The generation subproblem has become more complicated. However, due to our enumerative approach for solving the generation subproblem (see Section 3.2.3), only straightforward adjustments to the procedure are needed. The column generation procedure is implemented as described in Section 4.2 .5 (see also Section 3.4).

\subsubsection{Computational experiments}

We generated two sets of random instances. The first set was generated in the same way as the first set of Section 4.2.6. (and the first two sets of Section 3.5.1), except that all tools require just one slot in the tool magazine and each instance is described by $\mathrm{V}$ tool-job matrices. The values of $\mathrm{M}, \mathrm{N}, \mathrm{C}$ and $\mathrm{V}$, describing each instance type, are given in Table 4.6. 
Table 4.6 Parameters first set of instances

\begin{tabular}{|c|c|c|c|c|c|c||}
\hline $\begin{array}{c}\text { Problem size } \\
\text { M } \times \mathrm{N}\end{array}$ & $\mathrm{C}_{1}$ & $\mathrm{C}_{2}$ & $\mathrm{C}_{3}$ & $\mathrm{~V}$ & Min & Max \\
\hline $10 \times 10$ & 4 & 5 & 7 & 3 & 1 & 3 \\
$10 \times 10$ & 4 & 5 & 7 & 5 & 1 & 3 \\
$15 \times 20$ & 8 & 10 & 12 & 3 & 1 & 7 \\
$15 \times 20$ & 8 & 10 & 12 & 5 & 1 & 7 \\
$25 \times 30$ & 10 & 12 & 15 & 3 & 1 & 9 \\
$25 \times 30$ & 10 & 12 & 15 & 5 & 1 & 9 \\
$60 \times 40$ & 20 & 25 & 30 & 3 & 1 & 19 \\
$60 \times 40$ & 20 & 25 & 30 & 5 & 1 & 19 \\
\hline
\end{tabular}

The second set (see Table 4.7) consists of instances which have a block structure (that is, the tool requirements for subsets of jobs are interdependent) similar to the instances of the second dataset of Section 4.2.6. (or the third dataset of Section 3.5.1).

For these instances, the jobs are divided from the start in a number of feasible subgroups. First, a number $N_{1}$ is drawn uniformly between Minjob and Maxjob, and for each machine a subset of $\mathrm{C}$ tools is drawn. Then, the tool requirements on the different machines for the first $N_{1}$ jobs are generated using the same procedure as in Section 3.5.1, that is, making sure that these jobs form a feasible group. When $N_{1}$ jobs have been defined, then the procedure is iterated to produce $\mathrm{N}_{2}, \mathrm{~N}_{3}, \ldots$ additional jobs. The process stops when it is not possible to create a new group with Maxjob jobs. The last columns are filled independently of each other as for the first set of instances.

We considered two cases, with resp. 3 and 5 machines (see Table 4.6 and Table 4.7). For each instance type 10 instances were created (i.e. 30 or 50 tool job matrices had to be generated for each instance type) for a total of 300 instances.

\subsubsection{Computational results}

For a description of the software and hardware used we refer to Section 3.5.2. The results of the computational experiments are recorded in Tables $4.8,4.9$ and 4.10. The description of these tables is similar to that given in Section 4.2.7. (Table 4.8 (resp. 4.9 and 4.10 ) corresponds to Table 4.3 (resp. 4.4 and 4.5)). 
Table 4.7 Parameters second set of instances

\begin{tabular}{||c|c|c|c|c|c|c||}
\hline $\begin{array}{c}\text { Problem size } \\
\text { M } \times \text { N }\end{array}$ & $C_{1}$ & V & Min & Max & Minjob & Maxjob \\
\hline $30 \times 30$ & 15 & 3 & 5 & 8 & 4 & 7 \\
$30 \times 30$ & 15 & 5 & 5 & 8 & 4 & 7 \\
$40 \times 40$ & 20 & 3 & 7 & 10 & 5 & 8 \\
$40 \times 40$ & 20 & 5 & 7 & 10 & 5 & 8 \\
$50 \times 50$ & 25 & 3 & 8 & 12 & 6 & 10 \\
$50 \times 50$ & 25 & 5 & 8 & 12 & 6 & 10 \\
\hline
\end{tabular}

With the procedure sketched in Section 4.3.5. 296 out of 300 instances were solved to optimality. Another 2 instances were solved to optimality by using different parameters while for 2 instances a gap of one unit remained between the best lower bound $\mathrm{LB}_{\mathrm{CG}}$ and the best known upper bound on the solution value.

Table 4.8 shows that the lower bounds $\mathrm{LB}_{S W}$ and $\mathrm{LB}_{\mathrm{MSW}}$ are only sufficient for instances of type $\left(\mathrm{M}, \mathrm{N}, \mathrm{C}_{1}\right)$. The number of jobs in a group for these instances is extremely small (1 or 2). The performance of the sequential heuristics gets worse for instances with larger capacities. The MI and the Modified Rajagopalan rules outperform the other rules, although the MIMU, Whitney and Gaul, MU and Marginal gain rules give reasonable results. For the instances of the second set the performance of the Rajagopalan and the Marginal gain rules is terrible; this is certainly due to the nature of these rules, which select jobs having few tools in common with the jobs already chosen in a group. The best heuristic solution is in general very good, but for instances of type $\left(\mathrm{M}, \mathrm{N}, \mathrm{C}_{3}\right)$ the gap between heuristic and optimal solution value is often equal to 2 (see Table 4.8, column 12).

Table 4.9 shows that nearly all instances of the first set (of type (M, N, $\mathrm{C}_{1}$ )) can be solved in Step I of the procedure. For the remaining instances optimality is reached in Step III in many cases (73\%). For 27 percent of the instances Step IV is necessary. Table 4.9 shows that for 3 instances additional variables of the last set covering formulation were forced to take $0-1$ values to obtain an optimal solution, while 2 instances could not be solved to optimality. For these instances the maximal gap between the lower bound $\mathrm{LB}_{\mathrm{CG}}$ and the best known upper bound amounts to 1.19 . 
Table 4.8 Quality of lower and upper bounds

\begin{tabular}{|c|c|c|c|c|c|c|c|c|c|c|c|c|c|}
\hline \multicolumn{2}{|c|}{ Instance type } & \multirow[b]{2}{*}{$\begin{array}{c}\text { OPT } \\
1\end{array}$} & \multicolumn{3}{|c|}{ Lower bounds } & \multicolumn{8}{|c|}{ Upper bounds } \\
\hline $\begin{array}{c}M \times N \\
(V)\end{array}$ & $\mathrm{C}$ & & $\begin{array}{c}\mathrm{LB}_{\text {SW }} \\
2\end{array}$ & $\begin{array}{c}\text { LBMSW } \\
3\end{array}$ & $\begin{array}{c}\mathrm{LB}_{\mathrm{CG}} \\
4\end{array}$ & $\begin{array}{c}\text { MIMU } \\
5\end{array}$ & $\begin{array}{c}\text { MI } \\
6\end{array}$ & $\begin{array}{c}\mathrm{MU} \\
7\end{array}$ & $\begin{array}{l}\text { Whilney } \\
\text { and Gaul } \\
8\end{array}$ & $\begin{array}{c}\text { Rajagopa- } \\
\operatorname{lan} \\
9\end{array}$ & $\begin{array}{c}\text { Madified } \\
\text { Rajagopa- } \\
\text { lan } 10\end{array}$ & $\begin{array}{c}\text { Marginal } \\
\text { gain } \\
11\end{array}$ & $\begin{array}{c}\text { Best } \\
12\end{array}$ \\
\hline $10 \times 10$ & 4 & 7.5 & $0.0(10)$ & $0.0(10)$ & $0(10)$ & $0.0(10)$ & $0.0(10)$ & $0.0(10)$ & $0.0(10)$ & $0.2(8)$ & $0.0(10)$ & $0.0(10)$ & $0.0(10)$ \\
\hline & 5 & 4.8 & $-1.9(0)$ & $-1.3(0)$ & $0(10)$ & $0.3(9)$ & $0.3(9)$ & $0.4(8)$ & $0.3(9)$ & $0.6(6)$ & $0.3(9)$ & $0.3(9)$ & $0.2(8)$ \\
\hline (3) & 7 & 3.0 & $-1.0(0)$ & $-1.0(0)$ & $0(10)$ & $0.0(10)$ & $0.1(9)$ & $0.0(10)$ & $0.0(10)$ & $0.2(8)$ & $0.0(10)$ & $0.1(9)$ & $0.0(10)$ \\
\hline $10 \times 10$ & 4 & 9.1 & $0.0(10)$ & $0.0(10)$ & $0(10)$ & $0.0(10)$ & $0.0(10)$ & $0.0(10)$ & $0.0(10)$ & $0.0(10)$ & $0.0(10)$ & $0.0(10)$ & $0.0(10)$ \\
\hline & 5 & 5.0 & $-1.4(0)$ & $-1.1(1)$ & $0(10)$ & $0.4(9)$ & $0.5(8)$ & $0.3(10)$ & $0.4(9)$ & $0.6(7)$ & $0.5(8)$ & $0.4(9)$ & $0.3(7)$ \\
\hline (5) & 7 & 3.0 & $-1.0(0)$ & $-1.0(0)$ & $0(10)$ & $0.0(10)$ & $0.0(10)$ & $0.1(9)$ & $0.1(9)$ & $0.6(4)$ & $0.0(10)$ & $0.4(6)$ & $0.0(10)$ \\
\hline $15 \times 20$ & 8 & 14.8 & $0.0(10)$ & $0.0(10)$ & $0(10)$ & $0.2(10)$ & $0.2(10)$ & $0.4(8)$ & $0.3(9)$ & $0.3(9)$ & $0.2(10)$ & $0.4(8)$ & $0.2(8)$ \\
\hline & 10 & 7.6 & $-3.1(0)$ & $-3.0(0)$ & $0(10)$ & $0.8(8)$ & $0.6(10)$ & $1.1(5)$ & $0.7(9)$ & $1.9(0)$ & $0.6(10)$ & $1.6(2)$ & $0.6(4)$ \\
\hline (3) & 12 & 4.8 & $-2.7(0)$ & $-2.7(0)$ & $0(10)$ & $0.5(9)$ & $0.5(9)$ & $0.6(8)$ & $0.6(8)$ & $1.5(2)$ & $0.6(8)$ & $1.0(4)$ & $0.4(5)$ \\
\hline $15 \times 20$ & 8 & 18.4 & $0.0(10)$ & $0.0(10)$ & $0(10)$ & $0.2(8)$ & $0.0(10)$ & $0.2(8)$ & $0.1(9)$ & $0.0(10)$ & $0.0(10)$ & $0.0(10)$ & $0.0(10)$ \\
\hline & 10 & 9.3 & $-3.0(0)$ & $-2.8(0)$ & $0(10)$ & $0.8(6)$ & $0.5(9)$ & $1.2(3)$ & $0.7(7)$ & $1.1(3)$ & $0.5(9)$ & $1.1(5)$ & $0.4(5)$ \\
\hline$(5)$ & 12 & 5.3 & $-3.2(0)$ & $.3 .2(0)$ & $0(10)$ & $0.9(6)$ & $0.8(7)$ & $1.1(4)$ & $0.8(7)$ & $1.8(0)$ & $1.1(4)$ & $1.3(3)$ & $0.5(5)$ \\
\hline $25 \times 30$ & 10 & 21.8 & $-0.3(7)$ & $-0.2(8)$ & $0(10)$ & $0.3(8)$ & $0.3(8)$ & $0.6(5)$ & $0.4(7)$ & $0.7(6)$ & $0.3(8)$ & $0.3(8)$ & $0.1(9)$ \\
\hline & 12 & 13.6 & $-2.7(0)$ & $-2.6(0)$ & $0(10)$ & $1.8(3)$ & $1.2(8)$ & $2.0(3)$ & $1.6(5)$ & $1.6(4)$ & $1.2(8)$ & $1.8(3)$ & $1.0(2)$ \\
\hline (3) & 15 & 8.6 & $-6.2(0)$ & $-5.8(0)$ & $0(10)$ & $1.2(7)$ & $1.1(8)$ & $1.7(3)$ & $1.4(5)$ & $2.5(0)$ & $1.4(5)$ & $2.0(0)$ & $0.9(1)$ \\
\hline $25 \times 30$ & 10 & 25.9 & $-0.1(9)$ & $0.0(10)$ & $0(10)$ & $0.1(9)$ & $0.0(10)$ & $0.1(9)$ & $0.0(10)$ & $0.0(10)$ & $0.0(10)$ & $0.1(9)$ & $00(10)$ \\
\hline & 12 & 15.5 & $-2.2(4)$ & $-2.0(4)$ & $0(10)$ & $1.0(4)$ & $0.5(9)$ & $1.1(3)$ & $0.9(5)$ & $0.8(7)$ & $0.5(9)$ & $1.2(3)$ & $0.4(6)$ \\
\hline (5) & 15 & 9.5 & $-6.7(0)$ & $-6.3(0)$ & $0(10)$ & $1.6(6)$ & $1.5(6)$ & $1.9(4)$ & $1.5(7)$ & $3.0(0)$ & $1.5(6)$ & $2.4(0)$ & $1.1(1)$ \\
\hline $60 \times 40$ & 20 & 29.2 & $-0.1(0)$ & $-0.1(9)$ & $0(10)$ & $0.5(7)$ & $0.3(9)$ & $0.6(6)$ & $0.3(9)$ & $0.4(8)$ & $0.3(9)$ & $0.4(8)$ & $0.2(9)$ \\
\hline & 25 & 17.1 & $-11,2(0)$ & $-11.2(0)$ & $0(10)$ & $2.4(3)$ & $1.7(8)$ & $32(1)$ & $2.7(3)$ & $1.9(7)$ & $1.8(7)$ & $2.3(3)$ & $1.5(0)$ \\
\hline (3) & 30 & 12.2 & $-8.5(0)$ & $-8.3(0)$ & $0(10)$ & $2.3(6)$ & $1.8(10)$ & $2.9(1)$ & $2.2(7)$ & $2.9(1)$ & $1.9(9)$ & $2.8(1)$ & $1.8(0)$ \\
\hline $60 \times 40$ & 20 & 35.5 & $-0.1(9)$ & $-0.0(10)$ & $0(10)$ & $0.2(8)$ & $0.1(9)$ & $0.2(8)$ & $0.2(8)$ & $0.1(9)$ & $0.1(9)$ & $0.1(9)$ & $0.0(10)$ \\
\hline & 25 & 19.8 & $-10.7(0)$ & $-10.7(0)$ & $0(10)$ & $1.9(1)$ & $1.2(6)$ & $2.2(1)$ & $1.9(2)$ & $0.9(9)$ & $1.2(6)$ & $1.5(4)$ & $0.8(3)$ \\
\hline (5) & 30 & 13.8 & $-9.6(0)$ & $-9.4(0)$ & $0(10)$ & $2.5(3)$ & $1.9(9)$ & $2.8(2)$ & $2.5(3)$ & $2.7(2)$ & $1.8(10)$ & $2.8(2)$ & $1.8(0)$ \\
\hline $30 \times 30(3)$ & 15 & 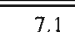 & $-5.1(0)$ & $-5.0(0)$ & $0(10)$ & $0.5(9)$ & $0.5(9)$ & $0.7(7)$ & $0.5(9)$ & $5.4(0)$ & $0.4(10)$ & $3.4(0)$ & $0.4(7)$ \\
\hline $40 \times 40$ (3) & 20 & 7.9 & $-5.9(0)$ & $-5.9(0)$ & $0(10)$ & $0.4(8)$ & $0.4(8)$ & $0.4(8)$ & $0.5(7)$ & $7.9(0)$ & $0.4(8)$ & $4.9(0)$ & $0.2(8)$ \\
\hline $50 \times 50(3)$ & 25 & 8.3 & $-6.3(0)$ & $-6.3(0)$ & $0(10)$ & $1.4(4)$ & $1.1(7)$ & $1.4(4)$ & $1.2(6)$ & $9.5(0)$ & $0.9(9)$ & $5.9(0)$ & $0.8(3)$ \\
\hline $30 \times 30(5)$ & 15 & 7.4 & $-5.3(0)$ & $-5.1(0)$ & $0(10)$ & $0.7(8)$ & $0.6(9)$ & $0.7(8)$ & $0.5(10)$ & $6.8(0)$ & $0.6(9)$ & $4.0(1)$ & $0.5(5)$ \\
\hline $40 \times 40(5)$ & 20 & 7.9 & $-5.9(0)$ & $-5.9(0)$ & $0(10)$ & $0.7(8)$ & $0.6(9)$ & $0.7(9)$ & $0,7(8)$ & $9.5(0)$ & $0.6(9)$ & $4.6(0)$ & $0.5(5)$ \\
\hline $50 \times 50(5)$ & 25 & 8.5 & $-6.5(0)$ & $-6.5(0)$ & $0(10)$ & $1.0(8)$ & $0.8(10)$ & $1.0(8)$ & $0.8(10)$ & $10.3(0)$ & $0.8(10)$ & $5.9(1)$ & $0.8(4)$ \\
\hline
\end{tabular}


Table 4.9 Performance of different steps of the column generation procedure

\begin{tabular}{|c|c|c|c|c|c|c|c|c|}
\hline $\begin{array}{r}\text { Instanc } \\
\mathrm{M} \times \mathrm{N}(\mathrm{V}) \\
\end{array}$ & $\mathrm{C}$ & Step I & Step II & $\begin{array}{c}\text { Step III } \\
\text { CG }\end{array}$ & $\begin{array}{c}\text { Step III } \\
\text { IP }\end{array}$ & $\begin{array}{c}\text { Step IV } \\
\text { A } \\
\end{array}$ & $\underset{B}{\text { Step IV }}$ & Gap \\
\hline $\begin{array}{c}10 \times 10 \\
(3)\end{array}$ & $\begin{array}{l}4 \\
5 \\
7 \\
\end{array}$ & 10 & & $\begin{array}{c}8 \\
10 \\
\end{array}$ & 2 & & & \\
\hline $\begin{array}{c}10 \times 10 \\
(5)\end{array}$ & $\begin{array}{l}4 \\
5 \\
7 \\
\end{array}$ & 10 & & $\begin{array}{c}7 \\
10 \\
\end{array}$ & 2 & 1 & & \\
\hline $\begin{array}{c}15 \times 20 \\
\text { (3) }\end{array}$ & $\begin{array}{c}8 \\
10 \\
12 \\
\end{array}$ & 8 & & $\begin{array}{l}4 \\
5 \\
\end{array}$ & $\begin{array}{l}2 \\
4\end{array}$ & $\begin{array}{l}2 \\
4 \\
\end{array}$ & & 1 \\
\hline $\begin{array}{c}15 \times 20 \\
5 \\
\end{array}$ & $\begin{array}{c}8 \\
10 \\
12 \\
\end{array}$ & 10 & & $\begin{array}{l}5 \\
5 \\
\end{array}$ & $\begin{array}{l}2 \\
2 \\
\end{array}$ & $\begin{array}{c}2 \\
2(1)\end{array}$ & & 1 \\
\hline $\begin{array}{c}25 \times 30 \\
\text { (3) }\end{array}$ & $\begin{array}{l}10 \\
12 \\
15 \\
\end{array}$ & 7 & & $\begin{array}{l}2 \\
2 \\
1\end{array}$ & $\begin{array}{l}1 \\
5 \\
1 \\
\end{array}$ & $\begin{array}{c}3 \\
7(1) \\
\end{array}$ & & \\
\hline $\begin{array}{c}25 \times 30 \\
\text { (5) }\end{array}$ & $\begin{array}{l}10 \\
12 \\
15 \\
\end{array}$ & $\begin{array}{c}10 \\
2\end{array}$ & & $\begin{array}{l}4 \\
1 \\
\end{array}$ & 3 & 1 & & \\
\hline $\begin{array}{c}60 \times 40 \\
(3) \\
\end{array}$ & $\begin{array}{l}30 \\
25 \\
30 \\
\end{array}$ & 8 & & 1 & $\begin{array}{l}1 \\
6 \\
1 \\
\end{array}$ & $\begin{array}{l}4 \\
9 \\
\end{array}$ & & \\
\hline $\begin{array}{c}60 \times 40 \\
(5)\end{array}$ & $\begin{array}{l}20 \\
25 \\
30\end{array}$ & 10 & & 3 & $\begin{array}{l}7 \\
1\end{array}$ & 6 & 3 & \\
\hline $\begin{array}{l}30 \times 30(3) \\
40 \times 40(3) \\
50 \times 50(3)\end{array}$ & $\begin{array}{l}15 \\
20 \\
25 \\
\end{array}$ & & & $\begin{array}{l}7 \\
8 \\
3\end{array}$ & $\begin{array}{l}2 \\
2 \\
6\end{array}$ & $\begin{array}{l}1 \\
1 \\
\end{array}$ & & \\
\hline $\begin{array}{l}30 \times 30(5) \\
40 \times 40(5) \\
50 \times 50(5)\end{array}$ & $\begin{array}{l}15 \\
20 \\
25 \\
\end{array}$ & & + & $\begin{array}{l}5 \\
5 \\
5 \\
\end{array}$ & $\begin{array}{l}5 \\
5 \\
4 \\
\end{array}$ & 1 & & \\
\hline \multicolumn{2}{|c|}{ All instances } & 75 & $0(+1)$ & 101 & 64 & $53(2)$ & 3 & 2 \\
\hline
\end{tabular}


Table 4.10 Computation times and size of problems

\begin{tabular}{|c|c|c|c|c|c|c|c|}
\hline \multicolumn{2}{|c|}{ Instance type } & \multicolumn{3}{|c|}{ Computation times (in seconds) } & \multirow[b]{2}{*}{$\begin{array}{c}\text { \#iterations } \\
\text { col.gen. instances } \\
4\end{array}$} & \multirow[b]{2}{*}{$\begin{array}{l}\text { \# columns } \\
\text { col. gen. instances } \\
5\end{array}$} & \multirow[b]{2}{*}{$\begin{array}{l}\text { maximal feasible } \\
\text { columns, all instances } \\
6\end{array}$} \\
\hline $\begin{array}{l}M \times N \\
(V)\end{array}$ & C & $\begin{array}{c}\text { Heuristics } \\
\text { all instances } \\
1\end{array}$ & $\begin{array}{l}\text { Sleps I-IV } \\
\text { all instances } \\
2\end{array}$ & $\begin{array}{l}\text { Steps I-IV } \\
\text { col. gen. instances } \\
3\end{array}$ & & & \\
\hline $\begin{array}{r}10 \times 10 \\
\text { (3) } \\
\end{array}$ & $\begin{array}{l}4 \\
5 \\
7 \\
\end{array}$ & $\begin{array}{l}2.1(2.0,2.3) \\
1.9(1.8,2.0) \\
1.8(1.8,1.9) \\
\end{array}$ & $\begin{array}{r}2.1(2.0,2.3) \\
13(6.3,20) \\
26(12.40) \\
\end{array}$ & $\begin{array}{r}\text { n.a. } \\
13(6.3,20) \\
26(12,40) \\
\end{array}$ & $\begin{array}{r}\text { n.a. } \\
2.5(1,4) \\
4.4(2,7) \\
\end{array}$ & $\begin{array}{r}\text { n.a. } \\
17(14,21) \\
30(22,38) \\
\end{array}$ & $\begin{array}{r}9(8,11) \\
30(25,34) \\
64(57,69) \\
\end{array}$ \\
\hline $\begin{array}{l}10 \times 10 \\
(5) \\
\end{array}$ & $\begin{array}{l}4 \\
5 \\
7 \\
\end{array}$ & $\begin{array}{l}3.3(3.2 .3 .5) \\
3.0(2.9,3.0) \\
2.7(2.7 .2 .8) \\
\end{array}$ & $\begin{array}{r}3.3(3.2,3.5) \\
14(8.1,24) \\
24(14,40) \\
\end{array}$ & $\begin{array}{r}\text { n.8. } \\
14(8.1,24) \\
24(14,40) \\
\end{array}$ & $\begin{array}{rr} & \text { n.a. } \\
2.2(1,4) \\
3.7(2.7) \\
\end{array}$ & $\begin{array}{r}\text { n.a. } \\
15(12,18) \\
25(19,29) \\
\end{array}$ & $\begin{array}{r}9(8,10) \\
26(20,34) \\
67(59,77) \\
\end{array}$ \\
\hline $\begin{array}{r}15 \times 20 \\
(3) \\
\end{array}$ & $\begin{array}{c}8 \\
10 \\
12 \\
\end{array}$ & $\begin{array}{r}11(10.11) \\
9.7(9.6,10) \\
9.1(9.0,9.2) \\
\end{array}$ & $\begin{array}{r}15(10,37) \\
52(27,75) \\
166(106,309) \\
\end{array}$ & $\begin{array}{r}30(23,37) \\
52(27,75) \\
166(106,309) \\
\end{array}$ & $\begin{array}{l}3.0(2,4) \\
4.6(2,8) \\
5.6(4,9) \\
\end{array}$ & $\begin{array}{r}20(19,20) \\
52(35,68) \\
143(117,173) \\
\end{array}$ & $\begin{array}{r}26(19,36) \\
124(97,166) \\
513(388,693) \\
\end{array}$ \\
\hline $\begin{array}{c}15 \times 20 \\
(5) \\
\end{array}$ & $\begin{array}{c}8 \\
10 \\
12 \\
\end{array}$ & $\begin{array}{l}18(17,18) \\
16(15,16) \\
14(14,15) \\
\end{array}$ & $\begin{array}{r}18(17,18) \\
49(26,85) \\
160(87,313) \\
\end{array}$ & $\begin{array}{r}\text { ก.2. } \\
49(26,85) \\
160(87,313) \\
\end{array}$ & $\begin{array}{r}\text { n.a. } \\
3.3(1,7) \\
4.7(3,9) \\
\end{array}$ & $\begin{array}{r}\text { n.a. } \\
37(29.43) \\
132(97,163) \\
\end{array}$ & $\begin{array}{r}19(17,20) \\
90(76,100) \\
384(306,469) \\
\end{array}$ \\
\hline $\begin{array}{c}25 \times 30 \\
(3)\end{array}$ & $\begin{array}{l}10 \\
12 \\
15\end{array}$ & $\begin{array}{l}37(35,38) \\
34(33,35) \\
31(30,31) \\
\end{array}$ & $\begin{array}{r}43(35,60) \\
11 !(58,164) \\
591(213.1095) \\
\end{array}$ & $\begin{array}{r}59(59,60) \\
111(58,164) \\
591(213,1095) \\
\end{array}$ & $\begin{array}{l}2.0(2,2) \\
4.3(2,8) \\
5.9(3,8)\end{array}$ & $\begin{array}{r}32(27,39) \\
80(41,136) \\
278(209,419) \\
\end{array}$ & $\begin{array}{r}55(32,79) \\
192(110,284) \\
1308(646,1997) \\
\end{array}$ \\
\hline $\begin{array}{c}25 \times 30 \\
(5) \\
\end{array}$ & $\begin{array}{l}10 \\
12 \\
15 \\
\end{array}$ & $\begin{array}{l}59(57,61) \\
53(52,56) \\
50(49,51)\end{array}$ & $\begin{array}{r}59(57,61) \\
99(54,170) \\
504(365,729) \\
\end{array}$ & $\begin{array}{r}\text { n.a. } \\
109(72,170) \\
504(365,729) \\
\end{array}$ & $\begin{array}{r}\text { n.a. } \\
3.4(1,5) \\
5.9(4,8) \\
\end{array}$ & $\begin{array}{r}\text { n.a. } \\
48(34,73) \\
259(150,384) \\
\end{array}$ & $\begin{array}{r}31(2637) \\
127(69,176) \\
806(445,1187) \\
\end{array}$ \\
\hline $\begin{array}{c}60 \times 40 \\
(3) \\
\end{array}$ & $\begin{array}{l}20 \\
25 \\
30 \\
\end{array}$ & $\begin{array}{l}141(139,143) \\
120(119,121) \\
117(116,118)\end{array}$ & $\begin{array}{r}154(140,227) \\
431(266,614) \\
1681(963,2626) \\
\end{array}$ & $\begin{array}{r}204(182,227) \\
431(266,614) \\
1681(963,2626) \\
\end{array}$ & $\begin{array}{l}3.0(2,4) \\
5.1(3,8) \\
6.6(4,8) \\
\end{array}$ & $\begin{array}{r}54(47,60) \\
173(105,252) \\
480(356,626) \\
\end{array}$ & $\begin{array}{r}97(70,164) \\
515(361,876) \\
2898(1852,4953) \\
\end{array}$ \\
\hline $\begin{array}{c}60 \times 40 \\
(5) \\
\end{array}$ & $\begin{array}{l}20 \\
25 \\
30 \\
\end{array}$ & $\begin{array}{l}230(228,232) \\
197(192,213) \\
191(190,193) \\
\end{array}$ & $\begin{array}{r}230(228,232) \\
422(248,661) \\
1343(795,1744) \\
\end{array}$ & $\begin{array}{r}\text { n.a. } \\
422(248,661) \\
1343(795,1744) \\
\end{array}$ & $\begin{array}{r}\text { n.a, } \\
4.0(1,7) \\
5.3(3,7) \\
\end{array}$ & $\begin{array}{r}\text { n.a. } \\
99(53,147) \\
288(221,413) \\
\end{array}$ & $\begin{array}{r}45(37,56) \\
240(189,269) \\
1223(860,1444) \\
\end{array}$ \\
\hline $\begin{array}{l}30 \times 30(3) \\
40 \times 40(3) \\
50 \times 50(3)\end{array}$ & $\begin{array}{l}15 \\
20 \\
25 \\
\end{array}$ & $\begin{array}{r}36(35,37) \\
81(80,82) \\
154(152,156) \\
\end{array}$ & $\begin{array}{r}240(149,398) \\
653(360,1730) \\
2895(824,7867) \\
\end{array}$ & $\begin{array}{r}240(149,398) \\
653(360,1730) \\
2895(824,7867) \\
\end{array}$ & $\begin{array}{r}7.9(5,13) \\
10.7(7,30) \\
18.5(7,41) \\
\end{array}$ & $\begin{array}{r}114(63,254) \\
158(85,236) \\
319(205,453) \\
\end{array}$ & $\begin{array}{r}752(497,1058) \\
2164(1655,2654) \\
7823(6230,10411)\end{array}$ \\
\hline $\begin{array}{l}30 \times 30(5) \\
40 \times 40(5) \\
50 \times 50(5)\end{array}$ & $\begin{array}{l}15 \\
20 \\
25 \\
\end{array}$ & $\begin{array}{r}58(57,59) \\
132(131,133) \\
249(247,251) \\
\end{array}$ & $\begin{array}{r}213(131,322) \\
710(319,1793) \\
3274(794,10386) \\
\end{array}$ & $\begin{array}{r}213(131,322) \\
710(319,1793) \\
3274(794,10386) \\
\end{array}$ & $\begin{array}{r}8.0(4,15) \\
11.8(7,21) \\
16.0(8,53) \\
\end{array}$ & $\begin{array}{r}63(43,107) \\
94(48,162) \\
271(143,431) \\
\end{array}$ & $\begin{array}{r}455(373,609) \\
1247(944,1556) \\
5341(4267,6568) \\
\end{array}$ \\
\hline
\end{tabular}


In Table 4.10 the computation times are given. It appears that only the instances of type $\left(\mathrm{M}, \mathrm{N}, \mathrm{C}_{3}\right)$ of the first set and the instances of the second set require much computational effort. But of course, these are the only really large ones (see column 6). Column 6 also shows that the instances of the first set are usually small. The instances of the second set are probably more realistic. The number of calls to LINDO was considerable for some of these instances (on average $8-19$, but with peaks of 53). The number of columns in the set covering formulation peaked at 453 for the instances of the second dataset.

For the 3-machine instances of type $\left(\mathrm{M}, \mathrm{N}, \mathrm{C}_{3}\right)$ of the first set and the instances of the second set the column generation approach proved to be a helpful tool in decreasing the size of the set covering formulation. For the instances of the second set the number of maximal feasible columns increased by a factor 10 from instance type $(30,30,15)$ to $(50,50,25)$. The increases in computation time were similar, but the number of columns in the LP subproblems only grew by a factor of 3 to $4 \frac{1}{2}$.

\subsection{Other extensions}

The extension of the job grouping problem to several non-identical machines is similar to the one described in Section 4.3. It is also possible to combine these changes for the case where jobs have to be processed on several non-identical machines with tools that require one or multiple slots in the tool magazines.

Rajagopalan (1985) and Hwang and Shogan (1989) discuss the introduction of due dates in the job grouping problem. Their models are sequential by nature and concentrate on finding one batch (optimal with respect to some "local" criterion) at a time. Due dates are incorporated by weighting jobs: jobs which have tight due dates receive larger weights and thus higher priority for being added to a next batch. By contrast the set covering formulation aims at finding a minimal number of groups, with no decisions taken on the order in which the groups have to be executed. Therefore the introduction of due dates does not fit very well into this formulation.

Similarly, the introduction of order quantities or production times would change the nature of the formulation. Because we should decide on the order in which groups are executed, additional variables should be added to incorporate this information.

A possible change in this direction is the introduction of separate weights for groups in different planning periods, where the weights depend on the planning period (each group (column) should be available in the 
formulation for each appropriate planning period). Additional constraints must then be added to the set covering formulation to prohibit that more than one group be assigned to some planning period (or that a job be executed more than once). The introduction of due dates also requires the separate evaluation of small groups. Previously, we only had to consider groups that were maximal (groups for which it is not possible to add jobs without destroying feasibility). Now, we must explicitly consider all groups that are not maximal, because different costs are attached to these smaller groups (increases in setup time can be traded off against decreases in the number of jobs that are overdue). As a result, the size of the formulation grows sharply with the number of possible planning periods.

This discussion shows that the set covering formulation is probably not the right tool to incorporate the introduction of due dates, production times and order quantities, and therefore we did not further investigate these extensions.

\subsection{Summary and conclusions}

In this study, the results of Chapter 3 have been extended to some special cases of the job grouping problem. First, the job grouping problem was considered in the case where tools need more than one slot in the tool magazine. Next, the job grouping problem for several identical machines was investigated. Lower and upper bounds were derived for these extensions. A column generation approach was used to compute a lower bound.

It appears that the lower bound obtained by computing the LP-relaxation value of the set covering formulation of the job grouping problem is very strong, though not always optimal. This is the case for both extensions studied. For 2 percent of the instances tested, this lower bound was strictly smaller than the best upper bound (gap equal to 1). In our computational experiments, the "multiple slots" instances tend to be more difficult and the "multiple machines" instances tend to be easier than the instances studied in Chapter 3.

\section{Acknowledgments}

We gratefully acknowledge useful discussions on the topic of this work with Antoon W.J. Kolen. We thank Ron van der Wal for solving some problems using a branch-and-bound procedure. 


\section{Chapter 5}

\section{A local search approach to job grouping}

\subsection{Introduction}

In Chapters 3 and 4, lower and upper bounding procedures were proposed for the job grouping problem. It appeared that, in many cases, sequential heuristic procedures were not sufficient to provide an optimal solution. A column generation approach was also developed to compute a strong lower bound, based on the linear relaxation of the set covering formulation of the job grouping problem. In the course of this procedure, it was sometimes possible to derive improved upper bounds. Notice, however, that solving the job grouping problem may be done faster if a better upper bound is known from the start. For instance, execution of the column generation procedure can be avoided if simple lower bounds like $\mathrm{LB}_{\mathrm{SW}}$ and $\mathrm{LB}_{\mathrm{MSW}}$ (see Chapters 3 and 4) are optimal and an optimal upper bound is also available. Alternatively, improved solutions provide additional columns to be included in the set covering formulation, which may speed up the column generation procedure.

Local search procedures may provide such improved solutions. Loosely speaking, a local search procedure tries to improve the current solution of a problem by searching the neighbourhood of that solution for improved solutions, until no better solution can be found, optimality is achieved or the procedure is stopped according to some other criterion. In this chapter, we investigate four possible ways for searching the neighbourhood, namely a simple improvement approach, a tabu search approach, a simulated annealing approach and a variable-depth approach, based on ideas of 
Kernighan and Lin (1970). For each of these methods three different objective functions and two different neighbourhood structures are considered for two different types of starting solutions. The environment in which these methods are applied is discussed in the next section. Section 5.3 contains the description of the methods. The results of our computational experiments are reported in Section 5.4. In this study, we consider instances where tools require several slots and instances with several machines. Some conclusions are drawn in Section 5.5.

\subsection{Local search environment}

Our objective is to partition the set of jobs into a minimal number of feasible groups, where a group is called feasible if the tools needed for the jobs in the group fit in the tool magazine of the machine(s). Our local search heuristics are designed to solve this problem indirectly, by answering a sequence of questions of the form: "Given a number $g$, is there a partition of the jobs into at most $g$ feasible groups?". This approach is motivated by the observation, made in Chapters 3 and 4 , that simple sequential heuristics provide good estimates for the optimal number of groups. For most instances tested the sequential heuristics provided a solution within 2 groups of the optimal solution.

Therefore, the local search procedure can be applied to find a solution which uses a number of groups equal to best known upper bound minus one. If a feasible solution is found the procedure is repeated for a smaller number of groups. If a solution is found with a number of groups equal to the lower bound the procedure ends. If we are not able to find a feasible solution for the given number of groups, the procedure is started again with a different starting solution. Other stopping criteria can be added to avoid endless runs. In this section we will discuss the setting of different parameters that are important for the execution of the local search procedures.

All procedures rely on the choice of a starting solution, an objective function $f(G)$ which evaluates the 'quality' of each solution $G$ (consisting of $g$ groups of jobs), a neighbourhood structure defining what solutions are considered as neighbours or perturbations of a given solution, and a stopping criterion. Before discussing all these concepts in detail, we first present, for illustration, the structure of an unsophisticated local search procedure to which we will refer as the simple improvement approach. The other local search procedures can be seen as variants of this basic strategy. 
Simple improvement approach (for finding a partition into $g$ feasible groups).

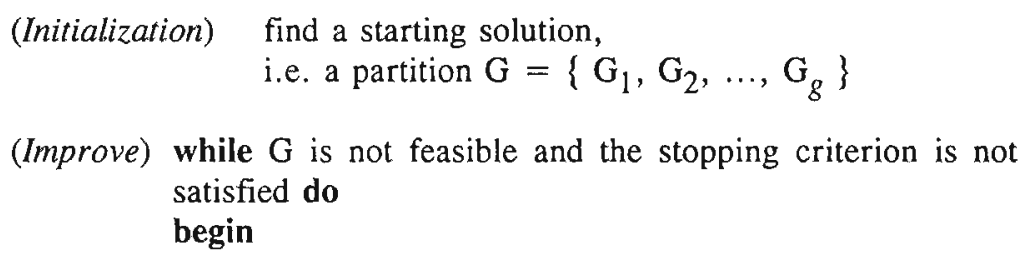

select a neighbour of $G$, say $G^{\prime}$, which minimizes $f\left(G^{\prime}\right)$ among all neighbours;

if $f\left(G^{\prime}\right) \geq f(G)$ and $G^{\prime}$ is not feasible

then return $F A I L$ (G is an infeasible local optimum)

else let $G:=G^{\prime}$

end

(Termination) if $\mathrm{G}$ is feasible

then return $\mathrm{G}$

else return $F A I L$ (stopping criterion satisfied)

\subsubsection{Starting solution}

A local search procedure starts from a given partition of the jobs into $g$ groups. This partition does not have to be feasible (if it is, we can stop the local search procedure for this number of groups). In our implementation we used two types of starting solutions, viz. the Maximal Intersection solution and a random starting solution. The Maximal Intersection (MI) starting solution is created using the Maximal Intersection rule for job grouping (see Sections 3.3.1, 4.2.4 and 4.3.4). Remember that this rule sequentially creates a number of groups. Here, we run the procedure until $g$ feasible groups are formed. Then, the remaining jobs (that are not (yet) assigned to some group) are distributed to the already created groups. This is done in a greedy way. The jobs are assigned to one of the $g$ groups in an arbitrary order, so that the sum of the violations of the tool magazine capacity constraint of the groups is minimized in each step (see next section).

A random starting solution is created by assigning jobs randomly to $g$ different groups. Some experiments were carried out using another structured starting solution (based on the Modified Rajagopalan rule, see Sections 3.3.1, 4.2.4 and 4.3.4), but these experiments offered no improvement over the MI starting solution. The MI starting solution was eventually preferred because the overall performance of the MI rule was better for the job grouping problem (see Chapters 3 and 4). 


\subsubsection{Objective function}

We used one of several measures to judge the quality of a partition of the jobs in a given number of groups.

\section{Minimize violations}

The violation $v(G)$ of a group $G$ of jobs is defined as the difference between the number of slots needed for the jobs in $G$ and the capacity of the tool magazine, if this difference is positive. The violation is zero if the tools fit in the tool magazine. The value of our first objective function is the sum of the violations of the groups. In case of several machines, we consider the sum of the violations over all machines. More precisely, define

$\mathrm{C}=$ capacity of the tool magazine,

$\mathrm{V}=$ number of machines,

$\mathrm{T}_{\mathrm{im}}=$ collection of tools necessary for job $\mathrm{i}$ on machine $\mathrm{m}$,

$s_{k}=$ number of slots necessary for tool $\mathrm{k}$,

$\mathrm{G}=$ collection of groups $\left(\mathrm{G}=\left\{\mathrm{G}_{1}, \mathrm{G}_{2}, \ldots, \mathrm{G}_{\mathrm{g}}\right\}\right)$,

$v\left(G_{j}\right)=$ violation of group $G_{j}$.

Then the violation of group $G_{j}(\in G)$ is $v\left(G_{j}\right)=\sum_{m=1}^{v} \max \{0$, $\left.\sum k \in\left\{U_{i \in G_{j}} T_{i m}\right\} s_{k}-C\right\}$. The objective function to be minimized is $f_{l}(G)=\sum G_{j} \in G \quad v\left(G_{j}\right)$. A feasible solution is found if the objective function is equal to 0 . This approach is derived from the approach of Chams, Hertz and de Werra (1987) for graph coloring.

2. Minimize violations \& maximize slack

Instead of only considering the violations, this objective function also takes into account the slack-capacity (i.e. the number of unused tool slots) in a group. It can be improved by increasing the slackcapacity in a group.

If $S_{j}$ is the slack $\left(S_{j}=\sum_{m=1}^{V} \max \left\{0, C-\sum k \in U_{i} \in G_{j} T_{i m} S_{k}\right\}\right)$ in a group $G_{j} \in G$, then the objective function to be minimized is $f_{2}(G)=\sum G_{j} \in G\left(C \cdot v\left(G_{j}\right)-S_{j}\right)$.

3. Maximize groupsize \& minimize violations

This objective function is inspired from the objective function of Johnson, Aragon, McGeoch and Schevon (1991) for graph coloring. The objective function is $f_{3}(G)=-\sum \mathrm{G}_{\mathrm{j}} \in \mathrm{G}\left|\mathrm{G}_{\mathrm{j}}\right|^{2}+$

$2 \sum G_{j} \in G\left|G_{j}\right| \cdot v\left(G_{j}\right)$. When minimizing this function, the first term tends to favour large groups, whereas the second term favours feasible groups. 


\subsubsection{Neighbourhood structure}

The neighbourhood structure defines the set of feasible moves from a certain solution. We studied two different neighbourhood structures:

1. Move and exchange

Given a solution, a neighbour solution is created by moving some job to another group or by exchanging two jobs. All possible moves and exchanges are considered in each iteration. To find a best move or exchange we visit all jobs consecutively. First, all possible moves for a job are considered. Then, all pairwise exchanges of the job with jobs in other groups are investigated. The best of these moves and exchanges is selected.

2. Move

Given a solution, a neighbour solution is created by moving some job to another group. We consider all moves for all jobs and select the best one. Compared to the first neighbourhood structure, the pairwise exchanges are not considered.

It is clear that the first neighbourhood structure uses more computation time per iteration then the other neighbourhood structure. On the other hand, the number of iterations to reach optimality will be decisive for the actual computational effort. In some procedures, the neighbourhood structure will be restricted by disallowing some moves or exchanges (see Section 5.3 on tabu-lists).

\subsubsection{Stopping criteria}

The local search procedure stops if feasibility is achieved or the computation time limit is reached. Use of a computation time limit is necessary to prevent some local search procedures from running endlessly. At the end of each step the solution is checked for feasibility since the values of the second and the third objective function give no conclusive evidence of feasibility of the solution.

For some local search approaches (viz. the simple improvement and the variable-depth approaches, see Section 5.3) the procedure is stopped when no improvements are possible any more (the tabu search always has the possibility to leave a local optimum, while the simulated annealing approach only stops if repeatedly no suitable neighbour solution can be found after a large number of trials; see Section 5.3.3). For randomly generated starting solutions an additional stopping criterion is added to 
allow testing of several starting solutions within a fixed amount of computation time. Namely, only a maximal number of steps can be performed from each starting solution, after which the procedure is restarted with a new random starting solution (here, a step is defined as a transition from one solution to another). In our implementation the procedure was restarted after $3 \cdot \mathrm{N}$ steps, where $\mathrm{N}$ is the number of jobs. This type of restart is not used with the MI starting solution, which thus can use the full amount of computation time.

\subsection{Local search approaches}

Four different approaches have been considered. The simple improvement approach only considers those moves which improve the solution at hand. The second approach (tabu search) also accepts moves that give a deterioration of the objective function. The third approach (simulated annealing) introduces a stochastic element in accepting moves that worsen the objective function value. In the fourth approach, ideas of Kernighan and Lin (1970) are implemented.

\subsubsection{Simple improvement approach}

This approach has been formally described in Section 5.2. Given the neighbourhood structure and the objective function, a move is accepted only if it improves the objective function. If it is not possible to find an improvement after all possible moves and/or exchanges have been considered the procedure stops.

\subsubsection{Tabu search approach}

The simple improvement approach follows a so-called hill climbing (or better valley seeking) approach for finding an optimum. The chances that the procedure gets stuck in a local optimum using the simple improvement approach are considerable. To overcome this difficulty, the tabu search approach allows moves that worsen the solution value. The idea is that accepting a number of 'bad' moves may open up possibilities to move to another (better) local optimum. The approach has achieved impressive practical successes for other combinatorial problems; for a thorough discussion we refer to Glover (1989; 1990).

When it is not possible to find an improved solution among the neighbours of the current one, a move is chosen that is the best among the 'bad' moves, that is a move that has the least impact on the value of the objective 
function. To avoid returning to a recent local optimum a tabu-list is used. More precisely, in our implementation, the tabu-list contains a list of jobs that are not allowed to be transferred to another group. The tabu-list may also contain a list of specified moves (in which explicitly the job, the old and the new group are recorded) that are not allowed, but preliminary tests showed no improvement over the current choice. We use a tabu-list of length 7 , which means that the last seven jobs that have been moved (in steps where the objective function deteriorated) may not be moved again (preliminary tests with a tabu-list of variable length - equal to $1 / 2 \cdot \mathrm{N}$, where $\mathrm{N}$ is the total number of jobs - led to a significant deterioration of the performance of the procedure). A move remains tabu only during a certain number of iterations, so that we have a cyclical list where the oldest forbidden move (or job) is removed whenever a new forbidden move (or job) is added. The procedure stops when a feasible solution is obtained or the computation time limit is reached.

To improve the performance of the procedure the concept of aspiration levels is introduced (see Glover (1989)). This offers the possibility of overriding the tabu status of a job (or move) on the tabu-list if the solution value which can be obtained by accepting this particular move is strictly smaller than the best known solution value.

\subsubsection{Simulated annealing approach}

Simulated annealing extends the simple improvement approach by allowing uphill moves during the minimization process, as in the tabu search approach (for a discussion on simulated annealing we refer to Van Laarhoven and Aarts (1987) and Johnson, Aragon, McGeoch and Schevon (1989; 1991)). However, the procedure of accepting uphill moves is randomized. If a move or exchange is selected in an iteration and this move offers an improvement of the objective function, the move is accepted. If it offers an increase of the objective function by $\Delta$, then the move is accepted with a probability of $\mathrm{e}^{-\Delta / T}$. The parameter $\mathrm{T}$ is referred to as temperature and is an important factor in the decision to accept uphill moves. If the temperature $T$ is large, the possibility of accepting 'bad' moves is large. Therefore, the temperature is gradually decreased in the course of the procedure ('annealing'). The behaviour of a simulated annealing implementation may be largely influenced by the setting of certain parameters, like the initial temperature and the cooling schedule. We used some suggestions of Johnson et al. $(1989 ; 1991)$ in our choices for these parameters; these are now as follows.

At the start of the simulated annealing procedure $T$ is fixed to some value $\mathrm{T}_{\text {START }}=4$. A number of iterations is carried out using this temperature. 
After MAXITER iterations have been performed at a certain temperature, the temperature is decreased. The parameter MAXITER is chosen proportional to the number of jobs $(\mathrm{N})$, viz. MAXITER $=3 \cdot \mathrm{N}$. The temperature $\mathrm{T}$ is decreased using geometric cooling (the temperature in a next step is $95 \%$ of the current temperature). To limit the time spent at high temperatures we introduce a parameter CUTOFF $(=0.3)$. This parameter makes sure that the temperature $T$ is decreased if too many moves are accepted. Thus, the temperature is decreased either after MAXITER moves or after CUTOFF . MAXITER accepted moves. Finally a parameter MINPERCENT $(=2 \%)$ is used as follows to decide whether a simulated annealing run can be stopped. A counter is incremented after each temperature change, if less than MINPERCENT of the selected moves have been accepted since the previous temperature change. The counter is reset to 0 whenever the current best solution is improved. If the counter reaches 5 , the process is declared frozen and stopped (see Johnson et al. (1989)). The procedure also stops if the computation time limit is reached. Finally, we introduced a tabu-list to avoid the possibility of returning too fast to an already visited local optimum. This tabu-list was implemented as in the tabu search approach (see Section 5.3.2).

\subsubsection{Variable-depth approach}

Kernighan and Lin (1970) proposed an effective heuristic algorithm for the graph partitioning problem. We use an idea similar to theirs. Each iteration of our procedure consists of a number of steps. In each step, a move or exchange is performed (the best one according to the given objective function and neighbourhood structure), and the jobs involved are placed on a tabu-list of length $\mathrm{N}$. After a number of steps, when all $\mathrm{N}$ jobs have been moved once (and placed on the tabu-list), a sequence of solutions has been obtained. From this sequence the best solution is chosen and a next iteration is performed starting from this solution. At the start of each new iteration all jobs are removed from the tabu-list (the tabu-list is emptied). The procedure is repeated until no improved solution can be found in an iteration or the maximum amount of computation time is used.

\subsection{Computational experiments}

Considering all combinations of two starting solutions, three objective functions, two different neighbourhood structures and four local search approaches, we get 48 possible implementations for a local search procedure (if we do not vary the other parameters). In this section we first 
make a selection among these methods, and then discuss the results of our computational experiments with the 'best' ones.

\subsubsection{The dataset}

Computational experiments were performed on a set of problem instances which emerged from the research described in Chapters 3 and 4. In these chapters, computational experiments were performed on a large set of randomly generated data (see Sections 3.6, 4.2.7 and 4.3.7). From this set, we selected a subset of instances for which the upper bounds computed by the sequential heuristics were not optimal (it is clear that this is not a random selection from the whole set of problems since all relatively easy instances were left out). The smallest instances were also discarded and, for each instance type $(M, N, C)$ (where $M$ is the number of tools, $N$ is the number of jobs and $\mathrm{C}$ is the capacity of the tool magazine), at most 5 problems were retained. We investigated three types of instances:

- single slot, single machine instances,

- multiple slot, single machine instances,

- single slot, multiple machine instances.

The dataset we used is described in Table 5.1. In each row the parameters for each instance type $(M, N, C)$ are given: $M, N, C$, the number of instances tested, the number of machines and the size of the tools. The upper part of Table 5.1 contains 45 instances of the single slot, single machine type (dataset 1 ). Then, 46 instances are described where tool sizes are tool-dependent (dataset 2). The last 54 instances are of the single slot, multiple machine type (dataset $3 \mathrm{~A}$ and $3 \mathrm{~B}$ ).

The sequential heuristics provided solutions close to optimality for all these instances (gap between upper bound and optimal solution is 1 or 2 ). Therefore, we tested all our local search heuristics by asking the question: "Is there a feasible solution involving exactly OPT groups?", where OPT was the optimal value of the instance at hand.

\subsubsection{Computational results}

The local search procedures were implemented in Turbo Pascal and run on an AT personal computer with $16 \mathrm{MHz} 80386 \mathrm{sx}$ processor and 80387 mathematical coprocessor (except for the results of Table 5.4; see below for details).

The computational experiments were performed as follows. First, extensive 
Table 5.1 Dataset local search

\begin{tabular}{|c|c|c|c|c|c|}
\hline Dataset & $\begin{array}{l}\text { Problem size } \\
\qquad \mathrm{M} \times \mathrm{N}\end{array}$ & C & $\begin{array}{l}\text { Number of } \\
\text { instances }\end{array}$ & $\begin{array}{l}\text { Number of } \\
\text { machines }\end{array}$ & $\begin{array}{l}\text { Number of } \\
\text { slots per tool }\end{array}$ \\
\hline $\begin{array}{c}\text { single slot } \\
\text { single machine } \\
\text { (dataset } 1 \text { ) }\end{array}$ & $\begin{array}{l}10 \times 10 \\
15 \times 20 \\
20 \times 30 \\
25 \times 30 \\
40 \times 30 \\
60 \times 40 \\
40 \times 40 \\
50 \times 50 \\
60 \times 60\end{array}$ & $\begin{array}{c}4 \\
8 \\
10 \\
10 \\
25 \\
30 \\
20 \\
25 \\
30 \\
\end{array}$ & $\begin{array}{l}5 \\
5 \\
5 \\
5 \\
5 \\
5 \\
5 \\
5 \\
5\end{array}$ & $\begin{array}{l}1 \\
1 \\
1 \\
1 \\
1 \\
1 \\
1 \\
1 \\
1\end{array}$ & $\begin{array}{l}1 \\
1 \\
1 \\
1 \\
1 \\
1 \\
1 \\
1 \\
1\end{array}$ \\
\hline $\begin{array}{l}\text { multiple slots } \\
\text { single machine } \\
\text { (dataset 2) }\end{array}$ & $\begin{array}{l}15 \times 20 \\
15 \times 20 \\
25 \times 30 \\
25 \times 30 \\
60 \times 40 \\
60 \times 40 \\
30 \times 30 \\
30 \times 30 \\
40 \times 40 \\
40 \times 40\end{array}$ & $\begin{array}{c}13,15 \\
13,15 \\
20 \\
25 \\
40 \\
45 \\
20 \\
20 \\
30 \\
30 \\
\end{array}$ & $\begin{array}{l}5 \\
5 \\
5 \\
4 \\
5 \\
5 \\
3 \\
5 \\
4 \\
5\end{array}$ & $\begin{array}{l}1 \\
1 \\
1 \\
1 \\
1 \\
1 \\
1 \\
1 \\
1 \\
1\end{array}$ & $\begin{array}{c}1,2,3 \\
1,3 \\
1,2,3 \\
1,3 \\
1,2,3 \\
1,3 \\
1,2,3 \\
1,3 \\
1,2,3 \\
1,3\end{array}$ \\
\hline $\begin{array}{c}\text { single slot } \\
3 \text { machines } \\
\text { (dataset } 3 \mathrm{~A} \text { ) }\end{array}$ & $\begin{array}{l}15 \times 20 \\
25 \times 30 \\
60 \times 40 \\
30 \times 30 \\
40 \times 40 \\
50 \times 50\end{array}$ & $\begin{array}{l}12 \\
15 \\
30 \\
15 \\
20 \\
25\end{array}$ & $\begin{array}{l}4 \\
5 \\
5 \\
3 \\
2 \\
5\end{array}$ & $\begin{array}{l}3 \\
3 \\
3 \\
3 \\
3 \\
3\end{array}$ & $\begin{array}{l}1 \\
1 \\
1 \\
1 \\
1 \\
1\end{array}$ \\
\hline $\begin{array}{c}\text { single slot } \\
5 \text { machines } \\
\text { (dataset } 3 \mathrm{~B} \text { ) }\end{array}$ & $\begin{array}{l}15 \times 20 \\
25 \times 30 \\
60 \times 40 \\
30 \times 30 \\
40 \times 40 \\
50 \times 50\end{array}$ & $\begin{array}{l}12 \\
15 \\
30 \\
15 \\
20 \\
25\end{array}$ & $\begin{array}{l}5 \\
5 \\
5 \\
5 \\
5 \\
5\end{array}$ & $\begin{array}{l}5 \\
5 \\
5 \\
5 \\
5 \\
5\end{array}$ & $\begin{array}{l}1 \\
1 \\
1 \\
1 \\
1 \\
1\end{array}$ \\
\hline
\end{tabular}


computational experiments were performed on the first dataset for a wide variety of implementations. The results of a number of approaches that performed relatively well are presented in Table 5.2. For this selection of approaches, additional experiments were performed on the instances of the second and the third datasets (for the "multiple slots" and the "multiple machines" instances).

The experiments on the first dataset were carried out with a 600 seconds limit on the computation time. Each instance was tested for a given starting solution, objective function, neighbourhood structure and local search approach. When a random starting solution was used a number of restarts was allowed within the given time period of 600 seconds (see Section 5.2.4).

Early tests showed that the simple improvement approach was dominated strongly by the other local search approaches, and therefore we did not consider this approach any further. The lack of good results for this strategy is probably due to the fact that the objective functions are such that the possibility of getting stuck in local optima (minima) is indeed large if no uphill moves are allowed.

The results for the other three local search approaches (i.e. tabu search, simulated annealing, variable-depth) did not diverge too much for a given starting solution, objective function and neighbourhood structure. Table 5.2 gives the results of the computational experiments for the tabu search approach using the objective functions $f_{l}(G), f_{2}(G), f_{3}(G)$ defined in Section 5.2.2 and the neighbourhood structures "move and exchange" and "move" described in Section 5.2.3. The second column of Table 5.2 indicates the number of instances tested for each instance type $(M, N)$ ( 5 for all instance types). Each entry of the table consists in a pair "A - B", where A describes the number of instances for which an optimal solution was found using the MI starting solution and $\mathrm{B}$ describes the number of instances that was solved using a random starting solution (possibly with multiple starts). The bottom line of the table gives the cumulative results.

The first two rows show that nearly all smaller instances can be solved by the tabu search approach, independently of the neighbourhood structure or the objective function. However, the results diverge for larger instances. It appears that the use of a MI starting solution gives better results than the implementation using a random starting solution. We come back to this issue in the discussion of Table 5.4. The second objective function, including the slacks, usually leads to the best results (an impression also 
Table 5.2 Results dataset 1: tabu search

\begin{tabular}{||c|c||c|c|c|c|c|c||}
\hline \hline \multirow{2}{*}{$\begin{array}{c}\text { Problem size } \\
\text { M } \times \text { N }\end{array}$} & \multirow{2}{*}{ Move and exchange } & \multicolumn{3}{c||}{ Move } \\
\cline { 3 - 8 } & & $f_{1}(G)$ & $f_{2}(G)$ & $f_{3}(G)$ & $f_{1}(G)$ & $f_{2}(G)$ & $f_{3}(G)$ \\
\hline \hline $10 \times 10$ & 5 & $5-5$ & $5-5$ & $5-5$ & $5-5$ & $5-5$ & $5-5$ \\
$15 \times 20$ & 5 & $5-5$ & $5-5$ & $4-5$ & $5-5$ & $5-5$ & $5-5$ \\
$20 \times 30$ & 5 & $4-2$ & $4-3$ & $4-2$ & $4-3$ & $5-4$ & $5-3$ \\
$25 \times 30$ & 5 & $1-1$ & $2-1$ & $1-0$ & $2-2$ & $3-4$ & $1-1$ \\
$40 \times 30$ & 5 & $5-4$ & $4-5$ & $5-3$ & $5-4$ & $5-5$ & $4-4$ \\
$60 \times 40$ & 5 & $2-1$ & $3-3$ & $2-0$ & $1-0$ & $2-2$ & $1-0$ \\
$40 \times 40$ & 5 & $2-1$ & $3-3$ & $3-4$ & $4-2$ & $4-4$ & $4-3$ \\
$50 \times 50$ & 5 & $4-0$ & $5-0$ & $3-0$ & $5-1$ & $5-2$ & $5-5$ \\
$60 \times 60$ & 5 & $2-0$ & $5-0$ & $3-0$ & $5-0$ & $4-0$ & $5-4$ \\
\hline Total & 45 & $30-19$ & $36-25$ & $30-19$ & $36-22$ & $38-31$ & $35-30$ \\
\hline
\end{tabular}

confirmed by our other experiments). The "move" neighbourhood provides slightly better results than the "move and exchange" neighbourhood. This may be due to the fact that the latter neighbourhood is computationally more expensive to explore and can perform fewer steps within a fixed time period. The results show that even large instances can be solved to optimality using a tabu search approach.

The trends discussed above for the tabu search approach have also been observed for the simulated annealing and the variable-depth approach. We do not give complete results for these two approaches, but limit ourselves to some brief comments. The results of the simulated annealing approach, though sometimes different for individual instances, are on average comparable to the tabu search results. The performance of the variabledepth approach is somewhat weaker (especially for objective functions $f_{1}(G)$ and $f_{3}(G)$ ), which may be due to the absolute stopping criterion used (see Sections 5.2.4 and 5.3.4). As an illustration of these comparisons, Table 5.3 reports on the results obtained by the three approaches on the first dataset, when the objective function $f_{2}(G)$ is used.

From these preliminary experiments, it appears that the objective function $f_{2}(G)$ conbined with the "move" neighbourhood provides the best results. For the variable-depth approach the more elaborate "move and exchange" neighbourhood provides better results, which may again be related to the influence of the stopping criterion (the time limit criterion is not often the reason to cut off the variable-depth search). In the remainder of this section 
Table 5.3 Selected results dataset 1

\begin{tabular}{||c|c||c|c|c|c|c|c||}
\hline \hline Problem size & \multirow{2}{*}{ \# } & \multicolumn{2}{|c|}{ Tabu search } & \multicolumn{2}{c|}{ Simulated Annealing } & \multicolumn{2}{c||}{ Variable - depth } \\
\cline { 3 - 8 } & & $\begin{array}{c}\text { Move and } \\
\text { exchange }\end{array}$ & Move & $\begin{array}{c}\text { Move and } \\
\text { exchange }\end{array}$ & Move & $\begin{array}{c}\text { Move and } \\
\text { exchange }\end{array}$ & Move \\
\hline \hline $10 \times 10$ & 5 & $5-5$ & $5-5$ & $5-5$ & $5-5$ & $5-5$ & $5-5$ \\
$15 \times 20$ & 5 & $5-5$ & $5-5$ & $5-5$ & $5-5$ & $3-5$ & $5-5$ \\
$20 \times 30$ & 5 & $4-3$ & $5-4$ & $4-1$ & $5-4$ & $5-3$ & $4-3$ \\
$25 \times 30$ & 5 & $2-1$ & $3-4$ & $2-1$ & $3-3$ & $2-1$ & $1-3$ \\
$40 \times 30$ & 5 & $4-5$ & $5-5$ & $4-5$ & $5-5$ & $3-5$ & $3-5$ \\
$60 \times 40$ & 5 & $3-3$ & $2-2$ & $3-2$ & $2-2$ & $3-3$ & $2-1$ \\
$40 \times 40$ & 5 & $3-3$ & $4-4$ & $3-3$ & $4-4$ & $4-2$ & $1-3$ \\
$50 \times 50$ & 5 & $5-0$ & $5-2$ & $5-0$ & $5-0$ & $5-0$ & $5-3$ \\
$60 \times 60$ & 5 & $5-0$ & $4-0$ & $5-0$ & $4-0$ & $5-0$ & $5-1$ \\
\hline Total & 45 & $36-25$ & $38-31$ & $36-22$ & $38-28$ & $35-24$ & $31-29$ \\
\hline \hline
\end{tabular}

we limit ourselves to the discussion of the objective function $f_{2}(G)$ combined with the "move and exchange" or the "move" neighbourhood.

Table 5.4 displays the influence of computation time when using a random starting solution within the tabu search framework, with the "move" neighbourhood structure. Of course, we expect the tabu search approach to give better results if the time limit is increased, but the extent of improvement is not clear. These experiments were run on a faster computer (with $25 \mathrm{Mhz} 80386$ processor), approximately twice as fast as the previous one. For each instance type $(\mathrm{M}, \mathrm{N}), 5$ instances were tested, and for each instance 25 random starting solutions were considered. Each column of Table 5.4 records the number of times (maximal 125) that an optimal solution was obtained within the given time limit (resp. 1, 5, 15, 30, 60, $150,300,450$ and 600 seconds).

The instances are roughly arranged by increasing size and the zeros in the lower diagonal part of Table 5.4 speak for themselves. Table 5.4 shows that the largest instances are indeed hard to solve using a random starting solution, especially if this performance is compared to the results using a MI starting solution (see Table 5.2 for the occurrence of optimality within 600 seconds on the slower computer). One may consider these computation times as very high, compared to the time it takes to solve similar instances to optimality (see Table 3.6, Chapter 3). These experiments were also carried out for the simulated annealing approach, with comparable results. 
Table 5.4 Results tabu search with random starting solution

\begin{tabular}{||c|c||c|c|c|c|c|c|c|c|c||}
\hline \hline \multirow{2}{*}{$\begin{array}{c}\text { Problem size } \\
\text { M }\end{array}$} & \multicolumn{6}{|c||}{ Cumulative number of instances solved to optimality after ... } \\
\cline { 3 - 11 } & & $1 \mathrm{~s}$ & $5 \mathrm{~s}$ & $15 \mathrm{~s}$ & $30 \mathrm{~s}$ & $60 \mathrm{~s}$ & $150 \mathrm{~s}$ & $300 \mathrm{~s}$ & $450 \mathrm{~s}$ & $600 \mathrm{~s}$ \\
\hline \hline $10 \times 10$ & 5 & 102 & 123 & 124 & 124 & 124 & 124 & 124 & 124 & 124 \\
$15 \times 20$ & 5 & 0 & 25 & 59 & 70 & 80 & 89 & 90 & 91 & 92 \\
$20 \times 30$ & 5 & 0 & 0 & 2 & 20 & 39 & 47 & 49 & 51 & 52 \\
$25 \times 30$ & 5 & 0 & 0 & 5 & 21 & 41 & 63 & 77 & 83 & 87 \\
$40 \times 30$ & 5 & 0 & 0 & 15 & 50 & 83 & 113 & 117 & 120 & 122 \\
$60 \times 40$ & 5 & 0 & 0 & 0 & 0 & 1 & 12 & 37 & 45 & 58 \\
$40 \times 40$ & 5 & 0 & 0 & 0 & 3 & 51 & 83 & 97 & 108 & 113 \\
$50 \times 50$ & 5 & 0 & 0 & 0 & 0 & 2 & 44 & 64 & 69 & 72 \\
$60 \times 60$ & 5 & 0 & 0 & 0 & 0 & 0 & 3 & 19 & 26 & 26 \\
\hline \hline
\end{tabular}

In Tables 5.5 and 5.6, a further comparison is made between the three local search approaches for the "multiple slots" and the "multiple machines" instances. As mentioned before, we choose to present the results of implementations using the superior objective function $f_{2}(G)$. Tests with the other objective functions yield results that are in general worse than the results obtained for this objective function, as previously illustrated in Table 5.2.

Table 5.5 Selected results dataset 2

\begin{tabular}{||c|c|c||c|c|c|c|c|c||}
\hline Problem size & \multirow{2}{*}{$\mathrm{H}$} & \multicolumn{2}{c|}{$\mathrm{s}_{\mathrm{k}} \in$} & \multicolumn{2}{|c|}{ Tabu search } & \multicolumn{2}{|c|}{ Simulated Annealing } & \multicolumn{2}{|c|}{ Variable - depth } \\
\cline { 3 - 9 } $\mathrm{M} \times \mathrm{N}$ & & & $\begin{array}{l}\text { Move and } \\
\text { exchange }\end{array}$ & Move & $\begin{array}{c}\text { Move and } \\
\text { exchange }\end{array}$ & Move & $\begin{array}{c}\text { Move and } \\
\text { exchange }\end{array}$ & Move \\
\hline \hline $15 \times 20$ & 5 & $1,2,3$ & $4-5$ & $5-5$ & $4-5$ & $5-5$ & $2-5$ & $2-5$ \\
$15 \times 20$ & 5 & 1,3 & $4-5$ & $5-5$ & $4-5$ & $5-5$ & $3-5$ & $3-5$ \\
$25 \times 30$ & 5 & $1,2,3$ & $2-2$ & $4-5$ & $2-4$ & $4-4$ & $3-3$ & $0-5$ \\
$25 \times 30$ & 4 & 1,3 & $4-3$ & $2-3$ & $4-2$ & $2-4$ & $1-3$ & $1-4$ \\
$60 \times 40$ & 5 & $1,2,3$ & $3-2$ & $3-3$ & $3-3$ & $3-3$ & $3-2$ & $2-3$ \\
$60 \times 40$ & 5 & 1,3 & $4-5$ & $5-5$ & $4-5$ & $5-5$ & $3-4$ & $3-5$ \\
$30 \times 30$ & 3 & $1,2,3$ & $2-2$ & $3-2$ & $2-2$ & $3-3$ & $1-2$ & $2-3$ \\
$30 \times 30$ & 5 & 1,3 & $3-4$ & $5-4$ & $3-4$ & $5-3$ & $2-2$ & $1-4$ \\
$40 \times 40$ & 4 & $1,2,3$ & $4-1$ & $2-0$ & $4-3$ & $2-0$ & $3-1$ & $2-0$ \\
$40 \times 40$ & 5 & 1,3 & $3-0$ & $1-1$ & $3-2$ & $1-0$ & $1-0$ & $1-0$ \\
\hline Total & 46 & & $33-29$ & $35-33$ & $33-35$ & $35-32$ & $22-27$ & $17-34$ \\
\hline
\end{tabular}

Table 5.5 displays the results for the "multiple slots" case (dataset 2). In 
the third column of Table 5.5 the size of the tools is given. Because the "multiple slots" instances are expected to be harder (see Chapter 4), the time limit is increased from 600 to 900 seconds (on the 'slower' computer). Objective function $f_{2}(G)$ combined with the "move" neighbourhood structure form the best parameter set for the "multiple slots" instances. The results obtained with a random starting solution are similar to those using the MI starting solution if the tabu search or simulated annealing approach is used. However, if the time limit stopping criterion is decreased from 900 to 600 seconds, the results for the random starting solution deteriorate much faster than for the MI starting solution. The total scores for the "move and exchange" neighbourhood change from $33-29,33$ - 35 and 22 - 27 (see last line of Table 5.5) to $33-24,32-25,22-25$ if the time limit is set to 600 seconds. The influence of a time limit reduction is also present (though less significantly) in case of the "move" neighbourhood. Thus, it seems that the relatively good results for the random starting solution are related to the large time limit. Table 5.5 also shows that the time limit of 900 seconds is probably not enough for the largest instances in case of a random starting solution.

The performance of the variable-depth approach is systematically worse than that of the other approaches if a MI starting solution is used. This may be partly explained by the stronger stopping criterion adopted (12 out of 24 (resp. 28 out of 29) unsolved instances for the variable-depth approach using a "move and exchange" (resp. "move") neighbourhood structure were stopped before the computation time limit was reached). The influence of the stopping criterion is largely decreased in case multiple random starts are used. The results for the variable-depth approach are comparable to the other results if a random starting solution is employed.

Table 5.6 records the results for the "multiple machines" instances (datasets 3 A \& B). The computations were performed using a 900 seconds time limit (in Chapter 4 it is shown that these instances are probably easier than those of the second dataset; however, in each step of the local search approach more function evaluations have to be made). The upper (resp. lower) part of Table 5.6 presents results on "3 machines" (resp. "5 machines") instances.

The tabu search and simulated annealing approaches give similar results, with the variable-depth approach trailing behind. The tests using a MI starting solution were more successful than those using a random starting solution, which may indicate that the time limit was too low for randomly generated starting solutions (compare with the results presented in Table 5.4). Nearly all structured instances (of sizes $(30,30),(40,40)$ and $(50$, 
Table 5.6 Selected results dataset $3 \mathrm{~A} \& 3 \mathrm{~B}$

\begin{tabular}{|c|c|c||c|c|c|c|c|c||}
\hline \hline Problem size & $\#$ & $\mathrm{~V}$ & \multicolumn{2}{|c|}{ Tabu search } & \multicolumn{2}{c|}{ Simulated Annealing } & \multicolumn{2}{c||}{ Variable - depth } \\
\cline { 3 - 8 } M $\times$ N & & & $\begin{array}{l}\text { Move and } \\
\text { exchange }\end{array}$ & Move & $\begin{array}{c}\text { Move and } \\
\text { exchange }\end{array}$ & Move & $\begin{array}{c}\text { Move and } \\
\text { exchange }\end{array}$ & Move \\
\hline \hline $15 \times 20$ & 4 & 3 & $4-4$ & $4-4$ & $4-4$ & $5-5$ & $4-4$ & $4-4$ \\
$25 \times 30$ & 5 & 3 & $3-4$ & $3-4$ & $3-4$ & $3-3$ & $3-3$ & $2-3$ \\
$60 \times 40$ & 5 & 3 & $2-1$ & $0-0$ & $2-0$ & $0-1$ & $1-1$ & $1-0$ \\
$30 \times 30$ & 3 & 3 & $3-3$ & $3-3$ & $3-3$ & $3-3$ & $2-3$ & $2-3$ \\
$40 \times 40$ & 2 & 3 & $2-0$ & $2-2$ & $2-1$ & $2-2$ & $2-0$ & $2-2$ \\
$50 \times 50$ & 5 & 3 & $5-0$ & $5-2$ & $5-0$ & $5-2$ & $5-0$ & $4-1$ \\
\hline $15 \times 20$ & 5 & 5 & $5-5$ & $4-5$ & $5-4$ & $4-4$ & $3-3$ & $3-5$ \\
$25 \times 30$ & 5 & 5 & $4-4$ & $2-2$ & $4-4$ & $2-2$ & $1-2$ & $1-0$ \\
$60 \times 40$ & 5 & 5 & $0-0$ & $0-0$ & $0-0$ & $0-0$ & $0-0$ & $0-0$ \\
$30 \times 30$ & 5 & 5 & $5-5$ & $5-5$ & $5-5$ & $5-5$ & $5-5$ & $4-5$ \\
$40 \times 40$ & 5 & 5 & $5-0$ & $5-3$ & $5-0$ & $5-5$ & $5-0$ & $5-4$ \\
$50 \times 50$ & 5 & 5 & $4-0$ & $5-1$ & $4-0$ & $5-2$ & $4-0$ & $5-1$ \\
\hline Total & 54 & $42-26$ & $38-31$ & $42-25$ & $39-34$ & $35-21$ & $33-28$ \\
\hline \hline
\end{tabular}

50)) were solved to optimality when a MI starting solution was used. The results were better for the "move and exchange" neighbourhood structure than for the "move" neighbourhood structure in case a MI starting solution was used. For random starting solutions the more time-efficient "move" neighbourhood structure was more appropriate.

\subsection{Summary and conclusions}

In this chapter, we investigated the use of local search approaches to improve the solution for the job grouping problem. Four local search approaches were considered, namely simple improvement, tabu search, simulated annealing and the variable-depth approach; for each of these methods, several starting solutions, objective functions, neighbourhood structures and stopping criteria were tested. Computational experiments using three sets of data seem to indicate that the latter choices considerably influence the performance of the different approaches, while the influence of the specific local search approach seems less serious as long as some kind of local optimum evading procedure is used. The opportunity to leave local optima is particularly important given the rigidity of some objective functions.

The differences in performance of the tabu search, simulated annealing and 
variable-depth approach are relatively small for the job grouping problem. In some cases the results for the variable-depth approach are a bit disappointing, but this may be partly related to the stopping criteria used (that is, the variable-depth approach does not always benefit from additional computation time as the other two approaches do).

Local search approaches are well known for their extensive use of computation time and, in this study, they live up to this expectation. However, initial solutions are sometimes improved in a limited amount of time, especially for smaller instances. The MI starting solution outperforms the random starting solutions in most cases. Since the MI starting solution can be quickly obtained, we find it advisable to use it as a starting point. The objective function $f_{2}(G)$, which combines minimizing the number of violations with increasing the slack in groups that have spare capacity, seems to be the most adequate objective function. Combined with either of the neighbourhood structures "move and exchange" or "move", it provided good results for all three datasets considered.

In conclusion, the experiments with local search approaches show that these approaches can be helpful in finding improved solutions for the job grouping problem. One important application could be the use of these methods for improving the initial set covering formulation of the job grouping problem by a column generation approach, as described in Chapters 3 and 4. 


\section{Chapter 6}

\section{Minimizing the number of tool switches on a flexible machine*}

\subsection{Introduction}

The importance of tool management for the efficient use of automated manufacturing systems has been recently stressed by several authors; we refer for instance to Gray, Seidmann and Stecke (1988) or Kiran and Krason (1988) for a thorough discussion of this issue. In particular, a central problem of tool management for flexible machines is to decide how to sequence the parts to be produced, and what tools to allocate to the machine, in order to minimize the number of tool setups. The problem becomes especially crucial when the time needed to change a tool is significant with respect to the processing times of the parts, or when many small batches of different parts must be processed in succession. These phenomena have been observed in the metal-working industry by Hirabayashi, Suzuki and Tsuchiya (1984), Finke and Kusiak (1987), Bard (1988), Tang and Denardo (1988a), Bard and Feo (1989), etc. Blazewicz, Finke, Haupt and Schmidt (1988) describe for instance an NC-forging machine equipped with two tool magazines, each of which can handle eight tools. The tools are very heavy, and exchanging them requires a sizeable fraction of the actual forging time. Another situation where minimizing the number of tool setups may be important is described by Förster and Hirt (1989, p. 109). These authors mention that, when the tool transportation

\footnotetext{
* An article based on this chapter will be published in The International Journal of Flexible Manufacturing Systems 6 (1), 1994.
} 
system is used by several machines, there is a distinct possibility that this system becomes overloaded. Then, minimizing the number of tool setups can be viewed as a way to reduce the strain on the tool transportation system. Bard (1988) mentions yet another occurrence of the same problem in the electronics industry. Suppose several types of printed circuit boards (PCBs) are produced by an automated placement machine (or a line of such machines). For each type of PCB, a certain collection of component feeders must be placed on the machine before boards of that type can be produced. As the machine can only hold a limited number of feeders, it is usually necessary to replace some feeders when switching from the production of one type of boards to that of another type. Exchanging feeders is a timeconsuming operation and it is therefore important to determine a production sequence for the board types which minimizes the number of "feedersetups". Identifying the feeders with tools, we see that this constitutes again an instance of the "job-sequencing and tool loading" problem evoked above.

The present paper deals with a particular formulation of this problem, due to Bard (1988) and Tang and Denardo (1988a). Suppose that a batch of N jobs have to be successively processed, one at a time, on a single flexible machine. Each job requires a subset of tools, which have to be placed in the tool magazine of the machine before the job can be processed. The number of tools needed to produce all the jobs in the batch is denoted by $\mathrm{M}$. We represent the data by an $\mathrm{MxN}$ tool-job matrix A, with:

$$
\begin{aligned}
\mathrm{a}_{\mathrm{ki}} & =1 \text { if job i requires tool } \mathrm{k}, \\
& =0 \text { otherwise, }
\end{aligned}
$$

for $\mathrm{k}=1,2, \ldots, \mathrm{M}$ and $\mathrm{i}=1,2, \ldots, \mathrm{N}$. Without loss of generality, A has no zero row. The tool magazine has a limited capacity: it can accommodate at most $\mathrm{C}$ tools, each of which fits in one slot of the magazine. To ensure feasibility of the problem, we assume that no job requires more than $\mathrm{C}$ tools. We also assume that, while the jobs are in process, the tool magazine is always loaded at full capacity (as will be explained below, this is in fact a non-restrictive assumption for our problem). We thus call any subset of $\mathrm{C}$ tools a loading of the magazine.

A job sequence is a permutation of $\{1,2, \ldots, N\}$, or, equivalently, of the columns of $\mathrm{A}$. As the number of tools needed to produce all jobs is generally larger than the capacity of the tool magazine (i.e., $\mathrm{M}>\mathrm{C}$ ), it is sometimes necessary to change tools between two jobs in a sequence. When this occurs, one or more tools are removed from the tool magazine and are 
replaced by a same number of tools retrieved from a storage area. We call setup the insertion of a tool in the magazine. A switch is the combination of a tool setup and a tool removal. Since each tool has to be set up at least once in order to process the whole batch of jobs, we will also pay attention to the extra setups of a tool, that is, to all setups of the tool other than the first one.

The tool switching problem is now defined as follows: determine a job sequence and an associated sequence of loadings for the tool magazine, such that all tools required by the $\mathrm{j}$-th job are present in the $\mathrm{j}$-th loading, and the total number of tool switches is minimized. In matrix terms, the tool switching problem translates as follows: determine an $\mathrm{MxN} \mathrm{0-1} \mathrm{matrix}$ $P=\left(p_{k j}\right)$, obtained by permuting the columns of $A$ according to some job sequence, and an $\mathrm{MxN} 0-1$ matrix $\mathrm{T}=\left(\mathrm{t}_{\mathrm{kj}}\right)$ containing $\mathrm{C}$ ones per column (each column of $T$ represents a tool loading), such that $t_{k j}=1$ if $p_{k j}=1$ (i.e., tool $\mathrm{k}$ is placed in the $\mathrm{j}$-th loading if it is needed for the $\mathrm{j}$-th job in the sequence; $k=1, \ldots \mathrm{M} ; j=1, \ldots, N)$, and the following quantity is minimized:

$$
\sum_{j=2}^{N} \sum_{k=1}^{M}\left(1-t_{k, j-1}\right) t_{k j}
$$

(this quantity is exactly the number of switches required for the loading sequence represented by $\mathrm{T}$ ). Observe that minimizing the number of tool switches is equivalent to minimizing the number of setups or of extra setups, since the following relations hold:

$$
\begin{aligned}
\text { number of setups } & =\text { number of switches }+\mathrm{C} \\
& =\text { number of extra setups }+\mathrm{M} .
\end{aligned}
$$

Let us now briefly discuss some of the (explicit and implicit) assumptions of the tool switching model.

(1) As mentioned before, the assumption that the tool magazine is always fully loaded does not affect the generality of the model. Indeed, since no cost is incurred for tools staying in the magazine, one may consider that the first $\mathrm{C}$ tools to be used are all incorporated in the very first loading; thereafter, a tool only needs to be removed when it is replaced by another one.

(2) Each tool is assumed to fit in one slot of the magazine. Removing this assumption would create considerable difficulties. For instance the 
physical location of the tools in the magazine would then become relevant, since adjacent slots would need to be freed in order to introduce a tool requiring more than one slot.

(3) The time needed to remove or insert each tool is constant, and is the same for all tools. This assumption is in particular crucial for the correctness of the KTNS procedure (see Section 6.2.2) which determines the optimal tool loadings for a given job sequence. Many of our heuristic procedures, however, can easily be adapted in the case where switching times are tool dependent.

(4) Tools cannot be changed simultaneously. This is a realistic assumption in many situations, e.g. for the forging or for the PCB assembly applications mentioned above.

(5) The subset of tools required to carry out each job is fixed in advance. This assumption could be relaxed by assuming instead that, for each job, a list of subsets of tools is given, and that the job can be executed by any subset in the list; (i.e., several process plans are given for each job; see e.g. Finke and Kusiak (1987)). Choosing the right subset would then add a new dimension (and quite a lot of complexity) to the problem.

(6) Tools do not break down and do not wear out. This assumption is justified if the tool life is long enough with respect to the planning horizon. Otherwise, one may want to lift the assumption "deterministically", e.g. by assuming that tool $\mathrm{k}$ is worn out after the execution of $w_{k}$ jobs, for a given value of $w_{k}$. Alternatively, breakdowns and wear may also be modelled probabilistically. This would obviously result in a completely new model.

(7) The list of jobs is completely known. This assumption is realistic if the planning horizon is relatively short.

This paper deals with various aspects of the tool switching problem. Section 6.2 contains some basic results concerning the computational complexity of this problem; in particular, we establish that the problem is already $N P$-hard for $\mathrm{C}=2$, and we present a new proof of the fact that, for each fixed job sequence, an optimal sequence of tool loadings can be found in polynomial time. In Section 6.3, we describe several heuristics for the tool switching problem, and the performance of these heuristics on randomly generated problems is compared in Section 6.4. Section 6.5 contains a summary of our results and presents perspectives for future research. The Appendix contains some graph-theoretic definitions. 


\subsection{Basic results}

We present in this section some results concerning the computational complexity of the tool switching problem. We assume that the reader is familiar with the basic concepts of complexity theory (see e.g. Nemhauser and Wolsey (1988)). Let us simply recall here that, loosely speaking, a problem is $N P$-hard if it is at least as hard as the traveling salesman problem (see the Appendix).

\subsubsection{NP-hardness results}

Tang and Denardo (1988a) claim that the tool switching problem is NPhard. They do not present a formal proof of this assertion, but rather infer it from the observation that the problem can be modelled as a traveling salesman problem with variable edge lengths. Our immediate goal will be to establish the validity of two slightly stronger claims.

Consider first the following restricted version of the tool switching problem:

Input: an $M \times N$ matrix $A$ and a capacity $C$.

Problem PI: $\quad$ is there a job sequence for $A$ requiring exactly $M$ setups (i.e., no extra setups)?

Theorem 6.1 Problem P1 is NP-hard.

Proof: It is straightforward to check that P1 is precisely the decision version of the so-called matrix permutation problem, which has been extensively investigated in the VLSI design literature (see Möhring (1990) and references therein). Several equivalent versions of the matrix permutation problem have been shown to be $N P$-hard (see Kashiwabara and Fujisawa (1979), Möhring (1990)), and hence Pl is NP-hard. Q.E.D.

In the description of problem $\mathrm{Pl}$, both $\mathrm{A}$ and $\mathrm{C}$ are regarded as problem data. But, from the viewpoint of our application, it may also be interesting to consider the situation where a specific machine, with fixed capacity, has to process different batches of jobs. The matrix A can then be regarded as the sole data of the tool switching problem. This observation leads us to define the following problem, where $\mathrm{C}$ is now considered as a fixed parameter:

Input: $\quad$ an $M \times N$ matrix $A$. 
Problem P2: $\quad$ find a job sequence for $A$ minimizing the number of setups required on a machine with capacity $C$.

Theorem 6.2 Problem $\mathrm{P} 2$ is $N P$-hard for any fixed $\mathrm{C} \geq 2$.

Proof: Let $\mathrm{G}=(\mathrm{V}, \mathrm{E}, \mathrm{d})$ be a graph and $\mathrm{H}=(\mathrm{E}, \mathrm{I}, \delta)$ be its edge-graph (see Appendix). We consider the problem of finding a minimal length TS path in $\mathrm{H}$ (problem P3 in the Appendix). We are now going to prove Theorem 6.2 by showing that this $N P$-hard problem can be formulated as a special case of problem $\mathrm{P} 2$, for any fixed $\mathrm{C} \geq 2$. For simplicity, we first concentrate on a proof of Theorem 6.2 for $\mathrm{C}=2$.

Let $\mathrm{V}=\{1,2, \ldots, \mathrm{M}\}$ and $\mathrm{E}=\left\{\mathrm{e}_{1}, \mathrm{e}_{2}, \ldots, \mathrm{e}_{\mathrm{N}}\right\}$. Define an $\mathrm{MxN}$ matrix $A$, with rows associated to the nodes of $G$, columns associated to the edges of $\mathrm{G}$, and such that:

$$
\begin{aligned}
& a_{k i}=1 \text { if edge } e_{i} \text { contains node } k \text {, } \\
& =0 \text { otherwise. }
\end{aligned}
$$

Consider now $\mathrm{A}$ as an instance of the tool switching problem, with capacity $\mathrm{C}=2$. A job sequence for this problem corresponds to a permutation of $\mathrm{E}$, and hence to a TS path in the edge-graph of G. Also, it is easy to see that the number of tool switches between two jobs $\mathrm{i}$ and $\mathrm{j}$, corresponding to the edges $e_{i}$ and $e_{j}$ of $G$, is:

- equal to 1 if $e_{i}$ and $e_{j}$ share a common node, that is, if $\delta\left(e_{i}, e_{j}\right)=1$ in $\mathrm{H}$;

- equal to 2 if $e_{i}$ and $e_{j}$ do not share a common node, that is, if $\delta\left(e_{i}, e_{j}\right)=$ $+\infty$ in $\mathrm{H}$.

This discussion immediately implies that an optimal job sequence for A (with capacity 2) always corresponds to a minimal length TS path in $\mathrm{H}$. Hence, we can solve P3 by solving P2, and this entails that P2 is NP-hard. To see that Theorem 6.2 is also valid for $\mathrm{C}>2$, it suffices to adapt the definition of $\mathrm{A}$ in the previous argument, by adding $\mathrm{C}-2$ rows of 1's to it; that is, $A$ now has $(M+C-2)$ rows, and $a_{k i}=1$ if $k \geq M+1$. The reasoning goes through with this modification.

Q.E.D.

\subsubsection{Finding the minimum number of setups for a fixed job sequence}

The tool switching problem naturally decomposes into two interdependent issues, namely:

(1) sequencing: compute an (optimal) job sequence, and

(2) tooling: for the given sequence, determine what tools should be loaded in the tool magazine at each moment, in order to minimize the total number of setups required. 
In their paper, Tang and Denardo (1988a) proved that the sequencing subproblem actually is the hard nut to crack, since the tooling problem can be solved in $\mathrm{O}(\mathrm{MN})$ operations by applying a so-called Keep Tool Needed Soonest (KTNS) policy. A KTNS policy prescribes that, whenever a situation occurs where some tools should be removed from the magazine, so as to make room for tools needed for the next job, then those tools which are needed the soonest for a future job should be removed last (we refer to Tang and Denardo (1988a) or Bard (1988) for a more precise description).

Tang and Denardo's proof of the correctness of KTNS relies on ad-hoc interchange arguments and is rather involved (as observed by Finke and Roger - see Roger (1990) - the correctness of KTNS was already established by Mattson, Gecsei, Slutz and Traiger (1970) in the context of storage techniques for computer memory, in the case where each job requires exactly one tool; their proof is similar to Tang and Denardo's). We now look at the tooling subproblem from a different angle, and show that the problem can be modelled as a specially structured 0-1 linear programming problem, which can be solved by a greedy algorithm due to Hoffman, Kolen and Sakarovitch (1985) (see also Nemhauser and Wolsey (1988), pp. 562 - 573; Daskin, Jones and Lowe (1990) present another application of the same greedy algorithm in a flexible manufacturing context). When translated in the terminology of the tool switching problem, this algorithm precisely yields KTNS. Thus, this argument provides a new proof of correctness for KTNS.

The bulk of the work in our derivation of the KTNS procedure will simply consist in reformulating the tooling problem in an appropriate form. With this goal in mind, we first introduce some new notations and terminology. For the remainder of this section, assume that the job sequence $\sigma$ is fixed. Let the $\mathrm{MxN}(0,1)$-matrix $\mathrm{P}$ be defined by:

$$
\begin{aligned}
\mathrm{p}_{\mathrm{ki}} & =1 \text { if tool } \mathrm{k} \text { is required for the } \mathrm{i} \text {-th job in } \sigma, \\
& =0
\end{aligned}
$$

(that is, $\mathrm{P}$ is obtained by permuting the columns of $\mathrm{A}$ according to the job sequence at hand). A tocling policy can now be described by flipping some entries of $P$ from 0 to 1 , until each column of $P$ contains exactly $C$ ones. If we denote by $c_{i}$ the remaining capacity of column $i$, that is the quantity:

$$
c_{i}=C-\sum_{k=1}^{M} p_{k i}
$$


then a tooling policy must flip $c_{i}$ entries from 0 to 1 in the $i$-th column of $\mathrm{P}$.

Let us next define a 0 -block of $\mathrm{P}$ as a maximal subset of consecutive zeroes in a row of $P$. More formally, a 0 -block is a set of the form $\{(k, i),(k, i$ $+1), \ldots,(\mathrm{k}, \mathrm{i}+\mathrm{j})\}$, for which the following conditions hold:

(1) $1<\mathrm{i} \leq \mathrm{i}+\mathrm{j}<\mathrm{N}$,

(2) $p_{k i}=p_{k, i+1}=\ldots=p_{k, i+j}=0$,

(3) $\mathrm{p}_{\mathrm{k}, \mathrm{i}-\mathrm{I}}=\mathrm{p}_{\mathrm{k}, \mathrm{i}+\mathrm{j}+1}=1$.

Intuitively, a 0-block is a maximal time interval before and after which tool $\mathrm{k}$ is needed, but during which it is not needed. It is easy to see that each 0block of $\mathrm{P}$ is associated with an extra setup of tool $\mathrm{k}$. Thus, flipping an element of $\mathrm{P}$ from 0 to 1 can only reduce the number of extra setups if this element belongs to a 0-block, and if all other elements of this 0-block are also flipped. In other words, only flipping whole 0-blocks can help reducing the number of setups.

Example 6.1 The matrix

$$
P=\left[\begin{array}{llllll}
0 & 1 & 0 & 0 & 1 & 1 \\
1 & 1 & 0 & 0 & 0 & 0 \\
1 & 0 & 1 & 1 & 0 & 1
\end{array}\right]
$$

contains three 0 -blocks, namely $\{(1,3),(1,4)\},\{(3,2)\}$ and $\{(3,5)\}$. They correspond to an extra setup of tool 1 in period 5, and two extra setups of tool 3 , in periods 3 and 6 . Assume that the capacity is $\mathrm{C}=2$. Then, the number of extra setups can be minimized by flipping the first and the third 0 -blocks to 1 , thus resulting in the matrix:

$$
T=\left[\begin{array}{llllll}
0 & 1 & 1 & 1 & 1 & 1 \\
1 & 1 & 0 & 0 & 0 & 0 \\
1 & 0 & 1 & 1 & 1 & 1
\end{array}\right]
$$

From the previous discussion, it should now be clear that the tooling problem can be rephrased as follows: flip to 1 as many 0-blocks of $\mathrm{P}$ as possible, while flipping at most $c_{i}$ entries in column $i(i=1,2, \ldots, N)$.

Denote by $B$ the number of 0 -blocks in $P$, and, for $j=1,2, \ldots, B$, introduce the decision variables: 


$$
\begin{aligned}
x_{j}=1 & \text { if the } \mathrm{j} \text {-th } 0 \text {-block is flipped to } 1, \\
=0 & \text { otherwise. }
\end{aligned}
$$

For $\mathrm{i}=1,2, \ldots, \mathrm{N}$ and $\mathrm{j}=1,2, \ldots, \mathrm{B}$, let also:

$$
\begin{aligned}
\mathrm{m}_{\mathrm{ij}} & =1 \text { if the } \mathrm{j} \text {-th } 0 \text {-block "meets" column } \mathrm{i} \text { in } \mathrm{P}, \\
& =0 \text { otherwise }
\end{aligned}
$$

(formally, a 0-block meets column $i$ if it contains an element of the form $(\mathrm{k}, \mathrm{i})$, for some $\mathrm{k}$; for instance, in Example 6.1, the first 0-block meets columns 3 and 4 ).

Now, the tooling problem admits the following $0-1$ linear programming formulation:

(TP) $\max \sum_{\mathrm{j}=1}^{\mathrm{B}} \mathrm{x}_{\mathrm{j}}$

subject to

$$
\begin{array}{ll}
\sum_{j=1}^{B} m_{i j} x_{j} \leq c_{i}, & i=1,2, \ldots, N \\
x_{j} \in\{0,1\}, & j=1,2, \ldots, B
\end{array}
$$

Assume now that the 0-blocks of $\mathrm{P}$ have been ordered in non-decreasing order of their "endpoints": that is, the 0-blocks of $\mathrm{P}$ have been numbered from 1 to $B$ in such a way that the index of the last column met by the $j$-th 0 -block is smaller than or equal to the index of the last column met by the $(j+1)$-st 0 -block, for $j=1, \ldots, B-1$. Then, the matrix $\left(m_{i j}\right)$ is a so-called greedy matrix, i.e. it does not contain the matrix $\left(\begin{array}{ll}1 & 1 \\ 1 & 0\end{array}\right)$ as a submatrix. Hoffman et al. (1985) considered the following, more general problem on an $\mathrm{NxB}$ greedy matrix:

(GP) $\quad \max \quad \sum_{\mathrm{j}=1}^{\mathrm{B}} \mathrm{b}_{\mathrm{j}} \mathrm{x}_{\mathrm{j}}$

subject to

$$
\sum_{j=1}^{B} m_{i j} x_{j} \leq c_{i} \quad i=1,2, \ldots, N
$$




$$
0 \leq \mathrm{x}_{\mathrm{j}} \leq \mathrm{d}_{\mathrm{j}} \quad \mathrm{x}_{\mathrm{j}} \text { integer } \quad \mathrm{j}=1,2, \ldots, \mathrm{B}
$$

where $b_{j}, d_{j}(j=1,2, \ldots, B)$ and $c_{i}(i=1,2, \ldots, N)$ are integers with $b_{1} \geq b_{2} \geq \ldots \geq b_{B}$. They proved that, when the matrix $\left(m_{i j}\right)$ is greedy, problem $(G P)$ can be solved by a greedy algorithm, in which each $\mathrm{x}_{\mathrm{j}}(\mathrm{j}=$ $1,2, \ldots, B)$ is successively taken as large as possible while respecting the feasibility constraints. Reformulating this algorithm for $(T P)$, we see that we should successively flip 0 -blocks to 1 , in order of nondecreasing endpoints, as long as the remaining capacity of all columns met by the 0 block is at least one. We leave it to the reader to check that this procedure is precisely equivalent to a KTNS policy.

Remark. In a more general situation where the setup times are not identical for all tools, the tooling subproblem can still be formulated as a problem of the form $(G P)$, where $\mathrm{b}_{\mathrm{j}}$ is now the time required to set up the tool associated with the $j$-th 0 -block. Since the condition $b_{1} \geq b_{2} \geq \ldots \geq$ $\mathrm{b}_{\mathrm{B}}$ does not generally hold for these setup times, the greedy algorithm of Hoffman et al. (1985) and KTNS are no longer valid. However, the matrix $\left(\mathrm{m}_{\mathrm{ij}}\right)$, being an interval matrix, is totally unimodular (see Section 6.3.4 and Nemhauser and Wolsey (1988) for definitions). It follows that the tooling subproblem can still be solved in polynomial time in that case, by simply solving the linear programming relaxation of the formulation $(G P)$.

\subsection{Heuristics}

The tool switching problem being $N P$-hard, and hence probably difficult to solve to optimality, we concentrate in the sequel on heuristic techniques for its solution. We propose hereunder six basic approaches, falling into two main categories (we adopt the terminology used by Golden and Stewart (1985) for the traveling salesman problem):

- construction strategies, which exploit the special structure of the tool switching problem in order to construct a single (hopefully good) job sequence (Subsections 6.3.1 to 6.3.4 below);

- improvement strategies, which iteratively improve a starting job sequence (Subsections 6.3.5 and 6.3.6 below).

Composite strategies will be obtained by combining construction and improvement procedures. A computational comparison of the resulting procedures will be presented in Section 6.4. 
As explained in Section 6.1, the data of our problem consist of an $\mathrm{MxN}$ tool-job matrix A and a capacity $\mathrm{C}$. We focus on the solution of the sequencing subproblem (see Subsection 6.2.2), since we already know that the tooling subproblem is easy to solve. Whenever we speak of the cost of a (partial) job sequence, we mean the minimal number of tool switches required by the sequence, as computed using KTNS.

\subsubsection{Traveling salesman heuristics}

These heuristics are based on an idea suggested by Tang and Denardo (1988a). They consider a graph $G=(V, E, ~ l b)$ (see Appendix for definitions), where $V$ is the set of jobs, $E$ is the set of all pairs of jobs, and the length $\mathrm{lb}(\mathrm{i}, \mathrm{j})$ of edge $\{i, j\}$ is an underestimate of the number of tool switches needed between jobs $\mathrm{i}$ and $\mathrm{j}$ when these jobs are consecutively processed in a sequence. More precisely:

$$
\operatorname{lb}(i, j)=\max \left(\left|T_{i} \cup T_{j}\right|-C, 0\right),
$$

where $T_{i}$ is the set of tools required by job $i(i=1,2, \ldots, N)$. Notice that, if each job requires exactly $\mathrm{C}$ tools (i.e. $\left|\mathrm{T}_{\mathrm{i}}\right|=\mathrm{C}$ for all $\mathrm{i}$ ), then $\mathrm{lb}(\mathrm{i}, \mathrm{j})$ is equal to the number of tool switches required between jobs $i$ and $j$ in any schedule.

Each traveling salesman (TS) path of $G$ corresponds to a job sequence for the tool switching problem. So, as suggested by Tang and Denardo (1988a), computing a short TS path in G constitutes a reasonable heuristic for the generation of a good sequence. As a matter of fact, when all jobs use full capacity, then the tool switching problem is precisely equivalent to the TS problem on $\mathrm{G}$.

In our computational experiments, we have considered the following procedures for constructing a short TS path in G:

(1) Shortest Edge heuristic: this is the heuristic used by Tang and Denardo (1988a), and called "greedy feasible" in Nemhauser and Wolsey (1988); complexity: $\mathrm{O}\left(\mathrm{N}^{2} \log \mathrm{N}\right)$;

(2) Nearest Neighbour heuristic with all possible starting nodes: see Golden and Stewart (1985), Johnson and Papadimitriou (1985); complexity: $\mathrm{O}\left(\mathrm{N}^{3}\right)$;

(3) Farthest Insertion heuristic with all possible starting nodes: see Golden and Stewart (1985), Johnson and Papadimitriou (1985); complexity: $\mathrm{O}\left(\mathrm{N}^{4}\right)$;

(4) $B \& B$ algorithm: this is a state-of-the-art branch-and-bound code, which 
solves TS problems to optimality: see Volgenant and Jonker (1982); complexity: exponential in the worst-case.

Procedures (1), (2) and (3) are well-known heuristics for the traveling salesman problem. In addition to the complexity mentioned for each procedure, an overhead of $\mathrm{O}\left(\mathrm{MN}^{2}\right)$ operations has to be incurred for the computation of the edge lengths $\mathrm{lb}(\mathrm{i}, \mathrm{j})$.

\subsubsection{Block minimization heuristics}

We describe now another way of associating a traveling salesman instance to any given instance of the tool switching problem. We first introduce a directed graph $D=\left(V^{*}, U, u b\right)$. Here, $V^{*}$ is the set of all jobs, plus an additional node denoted by 0 . Each ordered pair of nodes is an arc in $U$. The length $u b(i, j)$ of $\operatorname{arc}(i, j)$ is given by:

$$
\operatorname{ub}(i, j)=\left|T_{i}\right| T_{j} \mid,
$$

where $T_{i}$ is the set of tools required by job $i(i=1,2, \ldots, N)$, and $T_{0}$ is the empty set. In words, $u b(i, j)$ is the number of tools used by job $i$ but not by job $\mathrm{j}$; hence, $u b(i, j)$ is an upper bound on the number of tool switches between jobs $i$ and $j$, for any sequence in which $i$ and $j$ must be consecutively processed. If every job requires exactly $\mathrm{C}$ tools, then $\mathrm{ub}(\mathrm{i}, \mathrm{j})$ $=u b(j, i)=l b(i, j)$ is equal to the number of switches between $i$ and $j$. But in general, $u b(i, j)$ differs from $u b(j, i)$.

Each TS path of D finishing at node 0 defines a sequence of jobs, and the length of the path is an upper bound on the total number of switches entailed by the sequence. For reasons explained below, we refer to heuristics which attempt to construct a short TS path in D as block minimization heuristics. We have implemented two such heuristics:

(1) NN Block Minimization, based on a nearest neighbour heuristic with all possible starting nodes; complexity: $\mathrm{O}\left(\mathrm{N}^{3}\right)$;

(2) FI Block Minimization, based on a farthest insertion heuristic with all possible starting nodes; complexity: $\mathrm{O}\left(\mathrm{N}^{4}\right)$.

Let us mention another interesting interpretation of the block minimization approach. As in Section 6.2.2, consider the matrix $\mathrm{P}$ obtained after permuting the columns of $\mathrm{A}$ according to a job sequence $\sigma$. We define a $\mathrm{I}$ block of $\mathrm{P}$ as a set of entries, of the form $\{(\mathrm{k}, \mathrm{i}),(\mathrm{k}, \mathrm{i}+1), \ldots,(\mathrm{k}, \mathrm{i}+$ j)\}, for which the following conditions hold: 
(1) $1 \leq \mathrm{i} \leq \mathrm{i}+\mathrm{j} \leq \mathrm{N}$,

(2) $\mathrm{p}_{\mathrm{ki}}=\mathrm{p}_{\mathrm{k}, \mathrm{i}+\mathrm{i}}=\ldots=\mathrm{p}_{\mathrm{k}, \mathrm{i}+\mathrm{j}}=1$,

(3) either $\mathrm{i} \stackrel{=}{=}$ or $\mathrm{p}_{\mathrm{k}, \mathrm{i}-1}=0$,

(4) either $\mathrm{i}+\mathrm{j}=\mathrm{N}$ or $\mathrm{p}_{\mathrm{k}, \mathrm{i}+\mathrm{j}+1=0}$

(this definition does not exactly mimic the definition of 0-blocks, but the difference is irrelevant here). Notice that, were it not for the possibility to carry out KTNS on $\mathrm{P}$, then each 1-block of $\mathrm{P}$ would induce a tool setup in the job sequence $\sigma$. Thus, the number of 1-blocks of $P$ is an overestimate of the number of setups required by $\sigma$.

We leave it to the reader to check that the number of 1-blocks in $\mathrm{P}$ is also equal to the length of the TS path associated with $\sigma$ in D (and finishing at node 0 ). So, finding a shortest TS path in $\mathrm{D}$ is equivalent to determining a permutation of the columns of $\mathrm{A}$ which minimizes the number of 1-blocks in the permuted matrix. This observation is essentially due to Kou (1977). Kou (1977) also proved that finding a permutation which minimizes the number of 1-blocks is NP-hard (our proof of Theorem 6.2 establishes the same result). This justifies the use of heuristics in our block minimization approach.

\subsubsection{Greedy heuristics}

One of the obvious drawbacks of the heuristics described in Sections 6.3.1 and 6.3.2 is that they do not take a whole job sequence into account when estimating the number of tool switches required between pairs of jobs. For instance, $l b(i, j)$ is in general only a lower bound on the actual number of switches between jobs $i$ and $j$, and this lower bound can sometimes be a quite poor estimate of the actual value. An extreme case would arise when no job requires more than $C / 2$ tools; then, $l b(i, j)=0$ for each pair $(i, j)$, and any traveling salesman heuristic based on these edge-lengths picks a random job sequence! Similarly, ub(i, j) can also be a rough upper bound on the number of switches required. In order to alleviate this difficulty, we propose now the following (Simple) Greedy heuristic:

Step 1: $\quad$ start with the partial job sequence $\sigma=(1)$; let $Q=\{2,3, \ldots, N\}$.

Step 2: for each job.j in $\mathrm{Q}$, let $\mathrm{c}(\mathrm{j})$ be the cost of the partial sequence $(\sigma, \mathrm{j})$ (i.e., the number of tool switches entailed by this partial sequence, disregarding the remaining jobs).

Step 3: $\quad$ let $\mathrm{i}$ be a job in $\mathrm{Q}$ for which $\mathrm{c}(\mathrm{i})=\min _{\mathrm{j} \in \mathrm{Q}} \mathrm{c}(\mathrm{j})$; let $\sigma:=(\sigma, \mathrm{i})$ and $\mathrm{Q}:=\mathrm{Q} \backslash\{\mathrm{i}\}$. 
Step 4: $\quad$ if $\mathrm{Q}$ is not empty, then repeat Step 2;

else, stop with the complete sequence $\sigma$.

Greedy runs in time $\mathrm{O}\left(\mathrm{MN}^{3}\right)$, since it requires $\mathrm{O}\left(\mathrm{N}^{2}\right)$ applications of the KTNS procedure (in Step 2). Its empirical performance can be slightly improved by taking advantage of the fact that all the partial sequences considered in Step 2 share the same initial segment.

Of course, there is no mandatory reason to select job 1 first in Step 1 of Greedy, rather than any other job. This observation suggests to consider the following, more elaborate Multiple-Start Greedy heuristic: run $\mathrm{N}$ times Greedy, once for each initial sequence $\sigma=(j)(j=1,2, \ldots, N)$, and retain the best complete sequence found. This heuristic clearly dominates Greedy, in terms of the quality of the job sequence that it produces. Its worst-case complexity is $\mathrm{O}\left(\mathrm{MN}^{4}\right)$.

As a final note on this approach, it may be interesting to observe that, if each job requires exactly $C$ tools, then Multiple-Start Greedy is identical to the TS Nearest Neighbour heuristic (Section 6.3.1) or to the NN block minimization heuristic (Section 6.3.2).

\subsubsection{Interval heuristic}

In order to motivate our next heuristic, let us first consider a special situation: assume that the matrix $\mathrm{P}$ arising by permuting the columns of $\mathrm{A}$ according to some sequence $\sigma$ has precisely one 1-block in each row. In other words, the ones in each row of $\mathrm{P}$ occur consecutively. When this is the case we say that $\mathrm{A}$ is an interval matrix (or that $\mathrm{A}$ has the consecutive ones property; see e.g. Fulkerson and Gross (1965), Booth and Lueker (1976), Nemhauser and Wolsey (1988)). Then, the job sequence $\sigma$ requires only one setup per tool, and is obviously optimal.

Thus, every $\mathrm{MxN}$ interval matrix admits an optimal sequence with $M$ setups. Moreover, given an arbitrary matrix $\mathrm{A}$, one can decide in time $O(M N)$ whether $A$ is an interval matrix, and, in the affirmative, one can find within the same time bound a sequence entailing $M$ setups for $A$ (Booth and Lueker (1976) (notice that this does not contradict Theorem 6.1: by applying KTNS, a sequence with $M$ setups can sometimes be found for non-interval matrices). On the other hand, it is by no means clear that any of the heuristics described in Sections $6.3 .1,6.3 .2$ or 6.3 .3 would find an optimal job sequence for an interval matrix. 
These observations suggest the implementation of the following Interval heuristic. The heuristic simultaneously builds a "large" interval submatrix of $\mathbf{A}$, and computes an optimal job sequence for the submatrix. This sequence is the solution returned by the heuristic. More precisely:

Step l: $\quad$ initialize $\mathrm{K}=\{\}, \mathrm{k}=1$.

Step 2: determine whether the submatrix of A consisting of the rows with index in $\mathrm{K} \cup\{\mathrm{k}\}$ is an interval matrix;

if so, then let $\mathrm{K}:=\mathrm{K} \cup\{\mathrm{k}\}$ and let, $\sigma$ be an optimal job sequence for the submatrix; else, continue.

Step 3: $\quad$ if $\mathrm{k}<\mathrm{M}$, then let $\mathrm{k}:=\mathrm{k}+1$ and go to Step 2; else, continue.

Step 4: return the last job sequence found; stop.

The Interval heuristic has the attractive property that it produces an optimal job sequence for every interval matrix. The complexity of the heuristic is $\mathrm{O}(\mathrm{MN})$ if the algorithm by Booth and Lueker (1976) is used. In our implementation, we have used a slower, but simpler recognition algorithm for interval matrices, due to Fulkerson and Gross (1965).

In the following subsections, we concentrate on improvement strategies. The input for each procedure is some initial job sequence $\sigma$, that we subsequently attempt to improve in an iterative way.

\subsubsection{2-Opt strategies}

This class of strategies is based on an idea that has been widely used for other combinatorial optimization problems: given a sequence $\sigma$, try to produce a better sequence by exchanging two jobs in $\sigma$ (if $\mathrm{i}$ is the $\mathrm{n}$-th job and $j$ is the $p$-th job in $\sigma$, then exchanging $i$ and $j$ means putting $i$ in $p$-th position and $\mathrm{j}$ in $\mathrm{n}$-th position). We have considered two versions of this basic approach. The first one, called Global 2-Opt, can be described as follows:

Step 1: find two jobs $\mathrm{i}$ and $\mathrm{j}$ whose exchange results in an improved sequence; if there are no such jobs, then return $\sigma$ and stop; else, continue.

Step 2: exchange i and j; call $\sigma$ the resulting sequence; repeat Step 1.

Global 2-Opt has been proposed by Bard (1988) for the tool switching problem. Notice that each execution of Step 1 requires $\mathrm{O}\left(\mathrm{N}^{2}\right)$ applications of KTNS, i.e. $\mathrm{O}\left(\mathrm{MN}^{3}\right)$ operations. But the number of potential executions 
of this step does not appear to be trivially bounded by a polynomial in $\mathrm{N}$ and $M$ (contrary to what is claimed by Bard (1988)). In order to reduce the computational effort by iteration of Global 2-Opt, the following Restricted 2-Opt procedure can also be considered:

Step 1: find two consecutive jobs in $\sigma$, say the $\mathrm{p}$-th and $(\mathrm{p}+1)$-st ones, whose exchange results in an improved sequence; if there are no such jobs, then return $\sigma$ and stop.

Step 2: exchange the jobs found in Step 1; call $\sigma$ the resulting sequence; repeat Step 1.

The complexity of Step 1 in Restricted 2-Opt is $\mathrm{O}\left(\mathrm{MN}^{2}\right)$. This exchange strategy has also been proposed by Finke and Roger (see Roger (1990)).

\subsubsection{Load-and-Optimize strategy}

Consider again a job sequence $\sigma$ and the matrix $\mathrm{P}$ obtained by permuting the columns of A according to $\sigma$. Applying KTNS to $\mathrm{P}$ results in a new matrix $T$, each column of which contains exactly $\mathrm{C}$ ones (the $\mathrm{j}$-th column of $T$ describes the loading of the tool magazine while the $j$-th job in $\sigma$ is being processed). Suppose now that we look at $\mathrm{T}$ as defining a new instance of the tool switching problem (with capacity $\mathrm{C}$ ). If we can find for $\mathrm{T}$ a better sequence than $\sigma$, then this sequence will obviously be a better sequence than $\sigma$ for the original matrix $A$ as well. On the other hand, the problem instance $(T, C)$ is a little bit easier to handle than the instance (A,C). Indeed, since each column of $T$ contains $C$ ones, the tool switching problem $(\mathrm{T}, \mathrm{C})$ can be reformulated as a TS problem, as explained in Sections 6.3.1, 6.3.2, 6.3.3. These observations motivate our Load-andOptimize strategy:

Step 1: permute the columns of A according to $\sigma$ and apply KTNS; call $\mathrm{T}$ the resulting matrix.

Step 2: compute an optimal sequence $\sigma^{\prime}$ for the tool switching instance $(\mathrm{T}, \mathrm{C})$.

Step 3: if $\sigma^{\prime}$ is a better sequence than $\sigma$ for A, then replace $\sigma$ by $\sigma^{\prime}$ and repeat Step 1; else return $\sigma$ and stop.

From a practical viewpoint, we have found it easier to slightly alter this basic strategy, in the following way. In Step 2, rather than computing an optimal sequence for $\mathrm{T}$ (which is computational demanding), we simply use the farthest insertion heuristic to produce a good sequence $\sigma^{\prime}$ (as in Section 6.3.1). On the other hand, in Step 3, we accept the new sequence $\sigma^{\prime}$ even 
if it entails the same number of setups as $\sigma$. We only stop when 10 iterations of the procedure have been executed without producing a strictly improved sequence. In the sequel, we also refer to this variant as "Loadand-Optimize".

\subsection{Computational experiments}

\subsubsection{Generation of problem instances}

We tested our heuristics on 160 random instances of the tool switching problem. Of course, tool-job matrices occurring in practice may have characteristics not present in the ones we generated. For instance, as pointed out by an anonymous referee, realistic matrices are likely to display inter-row and inter-column correlations, as well as "tool clusters". However, in the absence of real-world data or even of detailed statistical information about these, we decided to follow a procedure similar to the one proposed by Tang and Denardo (1988a) in generating our test problems.

Each random instance falls into one of 16 instance types, characterized by the size $(\mathrm{M}, \mathrm{N})$ of the tool-job matrix and by the value $\mathrm{C}$ of the capacity. Accordingly, we denote the type of an instance by a triple (M, N, C). There are 10 instances of each type. The tool-job matrices are $\mathrm{MxN}$ matrices, where $(\mathrm{M}, \mathrm{N})$ is either $(10,10),(20,15),(40,30)$ or $(60,40)$. For each size $(M, N)$, we also define a pair (Min, Max) of parameters with the following interpretation:

- Min = lower bound on the number of tools per job,

- Max = upper bound on the number of tools per job.

The specific values of these parameters are displayed in Table 6.1.

For each problem size $(\mathrm{M}, \mathrm{N}), 10$ random matrices $\mathrm{A}$ were generated. For each $\mathrm{j}=1,2, \ldots, \mathrm{N}$, the $\mathrm{j}$-th column of $\mathrm{A}$ was generated as follows. First, an integer $t_{j}$ was drawn from the uniform distribution over [Min,Max]: this number denotes the number of tools needed for job $j$, i.e. the number of $I$ 's in the $j$-th column of $A$. Next, a set $T_{j}$ of $t_{j}$ distinct integers were drawn from the uniform distribution over $[1, \mathrm{M}]$ : these integers denote the tools required by job j, i.e. $a_{k j}=1$ if and only if $k$ is in $T_{j}$. Finally, we checked whether $\mathrm{T}_{\mathrm{j}} \subseteq \mathrm{T}_{\mathrm{i}}$ or $\mathrm{T}_{\mathrm{i}} \subseteq \mathrm{T}_{\mathrm{j}}$ held for any $\mathrm{i}<\mathrm{j}$. If any of these inclusions was found to hold, then the previous choice of $T_{j}$ was cancelled, 
Table 6.1 Parameter values Min and Max

\begin{tabular}{|c|c|c|}
\hline $\begin{array}{c}\text { Problem size } \\
\text { M } \times \text { N }\end{array}$ & Min & Max \\
\hline $10 \times 10$ & 2 & 4 \\
$20 \times 15$ & 2 & 6 \\
$40 \times 30$ & 5 & 15 \\
$60 \times 40$ & 7 & 20 \\
\hline
\end{tabular}

and a new set $T_{j}$ was generated (Tang and Denardo (1988a) and Bard (1988) have observed that any column of A contained in another column can be deleted without affecting the optimal solution of the problem; thus, we want to make sure that our problem instances actually involve $\mathrm{N}$ columns, and cannot be reduced by this simple trick). Notice that this generation procedure does not a priori prevent the occurrence of null rows in the matrix. In practice, only two of the 40 matrices that we generated contained null rows (these were two $(20,15)$ matrices, containing respectively one and three null rows).

A problem instance of type $(\mathrm{M}, \mathrm{N}, \mathrm{C})$ is now obtained by combining an $\mathrm{MxN}$ tool-job matrix $\mathrm{A}$ with one of the four capacities $\mathrm{C}_{1}, \mathrm{C}_{2}, \mathrm{C}_{3}$ and $\mathrm{C}_{4}$ displayed in Table 6.2.

Table 6.2 Problem sizes and tool magazine capacities

\begin{tabular}{||c|c|c|c|c||}
\hline $\begin{array}{c}\text { Problem size } \\
M \times N\end{array}$ & $C_{1}$ & $C_{2}$ & $C_{3}$ & $C_{4}$ \\
\hline $10 \times 10$ & 4 & 5 & 6 & 7 \\
$20 \times 15$ & 6 & 8 & 10 & 12 \\
$40 \times 30$ & 15 & 17 & 20 & 25 \\
$60 \times 40$ & 20 & 22 & 25 & 30 \\
\hline
\end{tabular}

We will see that the performance of some heuristics strongly depends on the value of the ratio $\mathrm{Max} / \mathrm{C}$. We call sparse those problem instances for which $\mathrm{Max} / \mathrm{C}$ is small, and dense those for which the ratio is close to 1 . Notice, in particular, that all instances of type $\left(\mathrm{M}, \mathrm{N}, \mathrm{C}_{1}\right)$ have $\mathrm{Max} / \mathrm{C}_{1}=$ 1. Varying the capacity as indicated in Table 6.2 will allow us to examine 
the behaviour of our heuristics under different sparsity conditions. Let us mention here that, according to the empirical observation of many realworld systems described by Förster and Hirt (1989), sparse instances are probably more "realistic" than dense ones. But of course, this conclusion is very much system-dependent.

\subsubsection{Computational results}

All heuristics described in Section 6.3 have been implemented in Turbo Pascal and tested on the problem instances described above. The experiments were run on an AT personal computer equipped with an $12 \mathrm{MHz}$ 80286 microprocessor and an additional 80287 coprocessor. Since our primary goal was to compare the quality of the solutions produced by the heuristics, no systematic attempts were made to optimize the running time of the codes. Accordingly, we will not report here on precise computing times, but simply give some rough indication of the relation between the times required by the various methods.

The performance of heuristic $\mathrm{H}$ on problem instance $\mathrm{I}$ is measured in terms of "percentage above the best solution found", namely, by the quantity:

$$
\delta_{\mathrm{H}}(\mathrm{I})=\left(\frac{\mathrm{H}(\mathrm{I})-\operatorname{Best}(\mathrm{I})}{\operatorname{Best}(\mathrm{I})}\right) * 100,
$$

where $\mathrm{H}(\mathrm{I})$ is the number of tool setups required by the job sequence produced by heuristic $\mathrm{H}$, and $\operatorname{Best}(\mathrm{I})$ is the number of setups required by the best sequence found by any of our heuristics.

For information, Table 6.3 indicates the evolution of Best(I) as a function of the problem type (average of Best (I) over all ten instances of each type). All subsequent tables (Tables $6.4,6.5,6.6$ ) report averages and (in brackets) standard deviations of $\delta_{\mathrm{H}}(\mathrm{I})$ over all instances I of a given type.

Table 6.4 compares the behaviour of the four traveling salesman heuristics described in Section 6.3.1. We will see later that TS heuristics perform best on dense instances, and tend to behave very badly on sparse instances. Therefore, we limit ourselves here to a comparison of these heuristics on the densest instances, that is, those instances where $C=C_{1}=$ Max.

From Table 6.4, it appears that on average, and mostly for large instances, Farthest Insertion yields better solutions than the other TS heuristics. 
Table 6.3 Average of Best (I)

\begin{tabular}{||c|c|c|c|c|}
\hline \multirow{2}{*}{$\begin{array}{c}\text { Problem size } \\
\mathrm{M} \times \mathrm{N}\end{array}$} & \multicolumn{4}{|c|}{ Tool magazine capacity } \\
\cline { 2 - 5 } $10 \times 10$ & $\mathrm{C}_{1}$ & $\mathrm{C}_{2}$ & $\mathrm{C}_{3}$ & $\mathrm{C}_{4}$ \\
\hline $20 \times 15$ & 26.5 & 11.2 & 10.3 & 10.1 \\
$40 \times 30$ & 113.6 & 21.6 & 20.0 & 19.6 \\
$60 \times 40$ & 211.6 & 189.7 & 76.8 & 56.8 \\
\hline
\end{tabular}

Table 6.4 Average (and standard deviation) of $\delta_{H^{(I)}}$ for the traveling salesman procedures

\begin{tabular}{|l|r|r|r|r||}
\hline Heuristic & $(10,10, \mathrm{C}=4)$ & $(20,15, \mathrm{C}=6)$ & $(40,30, \mathrm{C}=15)$ & $(60,40, \mathrm{C}=20)$ \\
\hline Shortest edge & $12.4(6.8)$ & $23.9(9.8)$ & $20.3(3.1)$ & $18.8(3.4)$ \\
Farthest Insertion & $12.1(9.8)$ & $15.5(8.6)$ & $9.4(5.3)$ & $6.9(2.7)$ \\
Nearest Neighbour & $13.7(7.8)$ & $19.8(7.7)$ & $21.0(6.0)$ & $18.9(3.5)$ \\
Branch-and-Bound & $12.6(4.6)$ & $16.2(5.8)$ & $12.4(4.3)$ & $10.9(2.9)$ \\
\hline
\end{tabular}

Farthest Insertion is also a very fast heuristic, which produces solutions in a matter of seconds (about 30 seconds for the largest instances). The Shortest Edge and Nearest Neighbour heuristics are even faster, but Farthest Insertion presents in our view the best quality $v s$. efficiency trade-off. Thus, we will select Farthest Insertion as our "winner" among TS heuristics, and no longer report on the other TS heuristics in the sequel.

A similar comparison between the two block minimization heuristics presented in Section 6.3.2 would lead to similar conclusions. Here again, FI is slightly better and slightly slower than NN. In the remainder of this section, we only report on the performance of FI, and no longer of NN.

Table 6.5 displays the performance of "constructive" and "improvement" heuristics over our complete sample of problem instances. The results (averages and standard deviations) for each heuristic are given in different columns. The results presented under the labels "2-Opt" or "Load-andOptimize" have been obtained by first picking a random job sequence, and then applying the corresponding improvement strategies to it. The columns labelled "Random" provide, for the sake of comparison, the number of tool setups entailed by the initial random job sequence. 
Let us now try to sketch some of the conclusions that emerge from this table. Consider first the case of dense instances, that is, the instances of type $(10,10,4),(20,15,6),(40,30,15)$ and $(60,40,20)$. As the size of these instances increases, the ranking of the solutions delivered by the various heuristics seems to become more or less stable. Namely, Multiple-Start Greedy and Global 2-Opt produce (on the average) the best results. Next comes a group made up of Farthest Insertion, FI Block Minimization and Simple Greedy, which usually yield solutions of slightly lower quality. Finally, the worst solutions are produced by Load-and-Optimize, Restricted 2-Opt and Interval (and, as expected, the random procedure).

We get a somewhat different ranking of the heuristics when we look at sparse instances. Consider e.g. the instances of type $(10,10,7),(20,15,12)$, $(40,30,25)$, (60,40,30). Multiple-Start Greedy, Global 2-Opt, Simple Greedy and FI Block Minimization remain, in that order, the best heuristics. But Farthest Insertion performs now almost as badly as the random procedure! As a matter of fact, for larger instances, it appears that the performance of Farthest Insertion deteriorates very systematically as sparsity increases. This behaviour is matched by all other TS heuristics (Shortest Edge, Nearest Neighbour, and B\&B). It can be explained by observing that, for sparse instances, the bounds $l b(i, j)$ tend to be poor estimates of the number of switches required between jobs $i$ and $j$ (see Sections 6.3.1 and 6.3.3).

Our conclusion at this point would be that, if we are only concerned with the quality of the solution produced by each heuristic, then Multiple-Start Greedy and Global 2-Opt come out the winners, while Simple Greedy and FI Block Minimization are good contenders. For dense problems, Farthest Insertion also is a very good technique.

This first picture becomes more nuanced when we also take computing times into account. Indeed, the various heuristics run at very different speeds. For instance, solving an instance of type $(10,10,4)$ takes about 0.30 seconds by Farthest Insertion, FI Block Minimization or by Simple Greedy, 2 seconds by Global 2-opt and 3 seconds by Multiple-Start Greedy. More strikingly, the instances of type $(60,40,20)$ require about 30 seconds by Farthest Insertion or by FI Block Minimization, 1.5 minutes by Simple Greedy, 30 minutes by Global 2-Opt, and 1 hour by Multiple-Start Greedy (these times are rather stable, for a given method, over all instances of the same type). Even though some of these procedures could certainly be accelerated by implementing them more carefully, it is probably safe to say that the first three heuristics are fast, while the latter two are computational 
Table 6.5 Average (and standard deviation) of $\delta_{\mathrm{H}}(\mathbf{1})$

\begin{tabular}{|c|c|c|c|c|c|c|c|c|c|c|}
\hline$M \times N$ & $c$ & $\begin{array}{l}\text { Farthest } \\
\text { Insertion }\end{array}$ & $\begin{array}{c}\text { FI Block } \\
\text { Minimization }\end{array}$ & $\begin{array}{l}\text { Simple } \\
\text { Greedy }\end{array}$ & $\begin{array}{l}\text { Multiple- } \\
\text { Start } \\
\text { Greedy }\end{array}$ & Interval & $\begin{array}{c}\text { Restricted } \\
2 \text {-opt }\end{array}$ & $\begin{array}{c}\text { Global } \\
2-o p t\end{array}$ & $\begin{array}{l}\text { Load-and- } \\
\text { Optimize }\end{array}$ & Random \\
\hline $10 \times 10$ & 4 & $12.1(9.8)$ & $14.3(7.7)$ & $12.3(6.3)$ & $4.6(3.8)$ & $22.6(12.2)$ & $26.0(7.7)$ & $8.7(4.7)$ & $5.8(5.3)$ & $41.2(18.9)$ \\
\hline $10 \times 10$ & 5 & $19.0(7.8)$ & $13.6(7.6)$ & $8.1(6.0)$ & $3.7(4.6)$ & $14.1(14.1)$ & $24.3(10.1)$ & $7.4(7.1)$ & $10.1(7.2)$ & $33.8(16.2)$ \\
\hline $10 \times 10$ & 6 & $17.8(10.8)$ & $9.7(6.4)$ & $5.7(4.7)$ & $2.9(4.4)$ & $9.7(11.8)$ & $18.3(7.7)$ & $3.0(4.6)$ & $6.7(4.4)$ & $26.3(9.1)$ \\
\hline $10 \times 10$ & 7 & $11.7(10.3)$ & $3.9(4.8)$ & $1.0(3.0)$ & $0.0(0.0)$ & $3.0(6.4)$ & $9.8(7.5)$ & $0.0(0.0)$ & $3.0(6.4)$ & $13.8(7.9)$ \\
\hline $20 \times 15$ & 6 & $15.5(8.6)$ & $12.0(4.2)$ & $13.7(7.0)$ & $4.6(3.5)$ & $25.7(9.7)$ & $33.6(7.2)$ & $10.0(4.3)$ & $12.3(6.8)$ & $45.9(8.8)$ \\
\hline $20 \times 15$ & 8 & $37.3(10.8)$ & $13.9(8.4)$ & $11.0(7.3)$ & $4.6(3.0)$ & $20.4(9.2)$ & $35.7(10.8)$ & $9.7(4.1)$ & $23.8(8.5)$ & $42.2(11.8)$ \\
\hline $20 \times 15$ & 10 & $30.5(5.8)$ & $8.3(6.2)$ & $5.6(4.3)$ & $1.5(2.3)$ & $10.4(8.2)$ & $24.3(9.2)$ & $6.4(7.3)$ & $25.6(11.7)$ & $30.1(12.3)$ \\
\hline $20 \times 15$ & 12 & $15.3(5.5)$ & $2.1(3.5)$ & $1.0(2.1)$ & $0.0(0.0)$ & $3.5(5.0)$ & $13.6(8.3)$ & $1.0(2.0)$ & $16.6(9.6)$ & $18.1(11.3)$ \\
\hline $40 \times 30$ & 15 & $9.4(5.3)$ & $8.8(4.4)$ & $11.4(4.8)$ & $6.2(3.1)$ & $30.5(4.3)$ & $30.3(5.0)$ & $6.0(4.0)$ & $16.6(5.3)$ & $42.9(6.1)$ \\
\hline $40 \times 30$ & 17 & $16.3(7.5)$ & $9.4(3.8)$ & $9.8(3.5)$ & $5.5(2.2)$ & $31.2(5.4)$ & $31.0(4.6)$ & $4.5(3.3)$ & $27.5(4.3)$ & $44.6(6.4)$ \\
\hline $40 \times 30$ & 20 & $33.8(9.1)$ & $12.1(3.6)$ & $9.8(4.2)$ & $3.2(2.0)$ & $30.4(6.0)$ & $33.0(6.6)$ & $6.0(2.9)$ & $35.1(6.4)$ & $45.5(8.9)$ \\
\hline $40 \times 30$ & 25 & $39.4(6.6)$ & $15.0(2.7)$ & $8.3(4.9)$ & $2.6(2.3)$ & $27.8(6.6)$ & $34.5(7.4)$ & $6.1(3.7)$ & $37.8(7.0)$ & $40.5(7.1)$ \\
\hline $60 \times 40$ & 20 & $6.9(2.7)$ & $9.7(2.4)$ & $10.2(2.6)$ & $5.8(1.5)$ & $30.6(2.7)$ & $25.8(3.8)$ & $4.8(2.4)$ & $20.0(3.8)$ & $37.1(3.6)$ \\
\hline $60 \times 40$ & 22 & $9.9(2.7)$ & $8.7(2.6)$ & $7.9(3.1)$ & $3.3(1.7)$ & $29.3(4.1)$ & $25.4(2.9)$ & $3.7(2.6)$ & $25.4(4.1)$ & $36.5(3.5)$ \\
\hline $60 \times 40$ & 25 & $21.8(5.7)$ & $10.5(3.1)$ & $8.2(2.8)$ & $2.8(2.0)$ & $30.2(3.6)$ & $29.7(3.0)$ & $2.1(1.9)$ & $35.5(4.3)$ & $38.0(3.6)$ \\
\hline $60 \times 40$ & 30 & $36.7(4.0)$ & $13.1(3.7)$ & $6.5(2.4)$ & $1.7(1.4)$ & $28.8(3.4)$ & $30.1(3.3)$ & $4.5(2.7)$ & $36.7(4.4)$ & $37.6(3.8)$ \\
\hline
\end{tabular}


more demanding. Therefore, for those applications where a solution of high quality has to be found quickly, FI Block Minimization and Simple Greedy seem to be perfectly adequate procedures (as well as Farthest Insertion, for dense instances). On the other hand, when computing time does not matter too much, and the thrust is instead on the quality of the solution, MultipleStart Greedy and Global 2-Opt could be considered.

Table 6.6 contains the results of our experiments with composite heuristics. The idea is here to quickly compute a good job sequence using one of the constructive heuristics, and to subsequently improve it by relying on some improvement strategy. In view of our previous experiments, we consider five ways to produce an initial solution (namely, by Farthest Insertion, FI Block Minimization, Simple Greedy, Interval and by a random procedure), and we choose Global 2-Opt as improvement strategy.

We see from Table 6.6 that, for dense instances, Farthest Insertion usually provides a very good initial solution, while FI Block Minimization always performs among the best for sparser instances. But in fact, surprisingly enough, all initialization procedures for Global 2-Opt (including the random one) come extremely close to each other, in terms of the quality of the solution produced. Also, their running times do not differ significantly.

\subsection{Lower bounds}

In order to judge of the quality of the heuristics described above, it would have been desirable to know tight and easily computed lower bounds on the cost of an optimal job sequence. The knowledge of such lower bounds would also be a prerequisite for the development of an exact optimization procedure (e.g. of the branch-and-bound type) for the tool switching problem. At this moment, unfortunately, we do not have very good lowerbounding procedures for our problem. We now briefly discuss some of the directions which may be worth exploring in this regard. In this discussion, we denote by cost $(\mathrm{A}, \mathrm{C})$ the total number of setups required by an optimal sequence for the problem instance $(A, C)$.

\subsubsection{Traveling salesman paths}

Since the quantity $\mathrm{lb}(\mathrm{i}, \mathrm{j})$ introduced in Section 6.3.1 is a lower bound on the number of tool switches incurred between job $\mathrm{i}$ and job $\mathrm{j}$ in any sequence, the length of a shortest TS path in the graph $G=(V, E, I b)$ certainly is a lower bound for the total number of switches in the optimal sequence (see Section 6.3.1). In other words, denoting by $L(A, C)$ the 
Table 6.6 Average (and standard deviation) of $\delta_{\mathrm{H}}(\mathrm{I})$ for composite heuristics

\begin{tabular}{|c|c|ccccc|}
\hline $\mathrm{M} \times \mathrm{N}$ & $\mathrm{C}$ & Farthest Insertion & $\begin{array}{c}\text { Fl Block } \\
\text { Minimization }\end{array}$ & Simple Greedy & Interval & Global 2-opt \\
\hline $10 \times 10$ & 4 & $5.0(5.5)$ & $8.7(6.9)$ & $5.4(3.6)$ & $6.9(4.5)$ & $8.7(4.7)$ \\
$10 \times 10$ & 5 & $8.3(5.3)$ & $7.3(7.1)$ & $5.3(5.5)$ & $3.6(4.4)$ & $7.4(7.1)$ \\
$10 \times 10$ & 6 & $4.9(4.9)$ & $2.9(4.4)$ & $1.9(3.8)$ & $2.0(4.0)$ & $3.0(4.6)$ \\
$10 \times 10$ & 7 & $2.0(4.0)$ & $1.0(3.0)$ & $0.0(0.0)$ & $1.0(3.0)$ & $0.0(0.0)$ \\
\hline $20 \times 15$ & 6 & $6.3(5.1)$ & $6.4(3.8)$ & $6.6(3.8)$ & $4.7(2.9)$ & $10.0(4.3)$ \\
$20 \times 15$ & 8 & $12.3(6.4)$ & $6.2(4.9)$ & $7.1(3.4)$ & $8.9(5.5)$ & $9.7(4.1)$ \\
$20 \times 15$ & 10 & $5.0(5.5)$ & $3.6(4.0)$ & $3.9(3.0)$ & $3.9(5.2)$ & $6.4(7.3)$ \\
$20 \times 15$ & 12 & $1.5(3.2)$ & $0.0(0.0)$ & $0.5(1.5)$ & $1.0(3.0)$ & $1.0(2.0)$ \\
\hline $40 \times 30$ & 15 & $2.5(3.1)$ & $2.8(2.0)$ & $5.3(4.3)$ & $5.3(3.1)$ & $6.0(4.0)$ \\
$40 \times 30$ & 17 & $3.1(1.3)$ & $3.0(2.5)$ & $5.0(2.4)$ & $6.5(2.6)$ & $4.5(3.3)$ \\
$40 \times 30$ & 20 & $6.6(4.1)$ & $3.4(2.1)$ & $5.3(2.7)$ & $6.6(2.9)$ & $6.0(2.9)$ \\
$40 \times 30$ & 25 & $7.7(3.0)$ & $3.9(2.2)$ & $4.6(3.4)$ & $9.1(5.1)$ & $6.1(3.7)$ \\
\hline $60 \times 40$ & 20 & $1.5(1.6)$ & $2.2(1.8)$ & $5.2(1.5)$ & $5.0(1.5)$ & $4.8(2.4)$ \\
$60 \times 40$ & 22 & $2.0(2.4)$ & $2.6(2.1)$ & $2.5(2.3)$ & $2.7(2.0)$ & $3.7(2.6)$ \\
$60 \times 40$ & 25 & $3.7(1.7)$ & $2.7(2.0)$ & $2.3(2.5)$ & $4.1(3.4)$ & $2.1(1.9)$ \\
$60 \times 40$ & 30 & $3.2(2.7)$ & $1.6(2.0)$ & $2.4(2.0)$ & $3.7(1.5)$ & $4.5(2.7)$ \\
\hline
\end{tabular}


length of such an optimal path, we see that $L(A, C)+C$ is a lower bound on cost (A, C) (Tang and Denardo (1988a)). Our computational experiments indicate that this bound is generally extremely weak.

The lower bound $\mathrm{L}(\mathrm{A}, \mathrm{C})+\mathrm{C}$ can sometimes be improved by relying on the following observations. It is obvious that, if $\left(A^{\prime}, C\right)$ is a new problem instance obtained by deleting some jobs from $A$ (i.e., the columns of $A^{\prime}$ form a subset of the columns of $A$ ), then the number of setups required for $\left(A^{\prime}, C\right)$ is never larger than the number of setups required for $(A, C)$, i.e. $\operatorname{cost}\left(\mathrm{A}^{\prime}, \mathrm{C}\right) \leq \operatorname{cost}(\mathrm{A}, \mathrm{C})$. Thus, in particular, $\mathrm{L}\left(\mathrm{A}^{\prime}, \mathrm{C}\right)+\mathrm{C}$ is a lower bound on cost $(\mathrm{A}, \mathrm{C})$. But it may happen that $\mathrm{L}(\mathrm{A}, \mathrm{C})<\mathrm{L}\left(\mathrm{A}^{\prime}, \mathrm{C}\right)$, in which case $L\left(A^{\prime}, C\right)+C$ is a sharper bound than $L(A, C)+C$.

Example 6.2 Consider the instance (A, C) described in Tang and Denardo (1988a). After some reductions, the instance involves 6 jobs, and the matrix $\mathrm{Ib}(\mathrm{i}, \mathrm{j})$ is given by :

$$
\mathrm{lb}=\left[\begin{array}{cccccc}
- & 2 & 3 & 2 & 2 & 1 \\
2 & - & 3 & 1 & 0 & 1 \\
2 & 3 & - & 3 & 2 & 2 \\
2 & 1 & 3 & - & 2 & 2 \\
2 & 0 & 2 & 2 & - & 2 \\
1 & 1 & 2 & 2 & 2 & -
\end{array}\right]
$$

The path $(3,6,1,4,2,5)$ is a shortest TS path with respect to $\mathrm{lb}$. Its length is $L(A, C)=6$. On the other hand, deleting the second job from this instance results in an instance ( $\left.A^{\prime}, C\right)$ for which the shortest Hamiltonian path $(3,6,1,4,5)$ has length $\mathrm{L}\left(\mathrm{A}^{\prime}, \mathrm{C}\right)=7$. Since the sequence $(3,6,1,2,4,5)$ requires precisely 7 switches (see Tang and Denardo (1988a)), we conclude that this sequence is optimal for the instance $(A, C)$.

An interesting question is how the partial instance $\left(A^{\prime}, C\right)$ should be (heuristically) picked in order to raise as much as possible the lower bound $L\left(A^{\prime}, C\right)+C$. This question has not been investigated yet.

\subsubsection{Structures implying extra setups}

Another approach for obtaining lower bounds on cost (A, C) is to identify subsets of tools for which extra setups are needed in any sequence. This can for instance be done as follows. Let $\mathrm{K}$ be a subset of rows (viz. tools), and $J$ a subset of columns (viz. jobs) of A. Say that a job $j \in J$ is heavy (with respect to $\mathrm{J}$ and $\mathrm{K}$ ) if, for every partition of $\mathrm{J}$ into $\mathrm{J}_{1} \cup\{\mathrm{j}\} \cup \mathrm{J}_{2}\left(\mathrm{~J}_{1}\right.$ 
and $\mathrm{J}_{2}$ nonempty),

$$
\begin{aligned}
& \left|\left\{k \in K: a_{k j}=1\right\}\right|+\mid\left\{k \in K: a_{k j}=0 \text { and } a_{k r}=a_{k s}=1\right. \\
& \text { for some } \left.r \in J_{1}, s \in J_{2}\right\} \mid>C \text {. }
\end{aligned}
$$

The idea behind this definition is easy to grasp : the left-hand side of (6.1) represents the number of tools required to process job $\mathrm{j}\left(\mathrm{a}_{\mathrm{kj}}=1\right)$, plus the number of tools which are not required by $\mathrm{j}\left(\mathrm{a}_{\mathrm{kj}}=0\right)$, but which are used before and after $j$ in a sequence of the form $\left(\jmath_{l}, j, J_{2}\right)\left(a_{k r}=a_{k s}=1\right)$. Now, we have :

Theorem 6.3 If $\mathrm{J}$ contains three heavy jobs with respect to $\mathrm{J}$ and $\mathrm{K}$, then, in any sequence, at least one tool of $\mathrm{K}$ incurs an extra setup.

Proof: Consider any sequence. We can always find a partition $\mathrm{J}$ into $\mathrm{J}_{1} \cup$ $\{\mathrm{j}\} \cup \mathrm{J}_{2}$ such that $\mathrm{j}$ is heavy, $\mathrm{J}_{1}$ and $\mathrm{J}_{2}$ are nonempty, all the jobs in $\mathrm{J}_{1}$ occur before $j$ in the sequence, and all the jobs in $J_{2}$ occur after $j$. It follows directly from (6.1) that, among all the tools of $\mathrm{K}$ which are needed both in $\mathrm{J}_{1}$ and in $\mathrm{J}_{2}$, some will not be present in the magazine when job $\mathrm{j}$ is processed (since this would exceed the magazine capacity). Hence, an extra setup will be necessary for these tools.

Q.E.D.

The statement of Theorem 6.3 is probably too general to be of direct interest. But it can nevertheless be used to identify some substructures in the tool-job matrix A which imply extra setups in any sequence. Two illustrations of such structures are now given.

1) Assume there exist three jobs (say, without loss of generality, $\mathrm{j}=1$, 2,3 ) and a subset $K$ of tools such that :

(i) each tool in $\mathrm{K}$ is used by exactly two of the jobs 1, 2, 3;

(ii) each of the jobs 1, 2, 3 needs (strictly) more than $\mathrm{C}-\mathrm{K}$ tools among those not in $\mathrm{K}$.

Under these conditions, one verifies that the jobs 1, 2, 3 are heavy with respect to $\mathrm{K}$; hence, the conclusion of Theorem 6.3 applies.

2) Suppose that $J$ and $K$ are subsets of jobs and tools respectively, such that :

(i) $\quad|\mathrm{J}|=|\mathrm{K}| \geq 3$;

(ii) the submatrix of $\mathrm{A}$ with column-set $\mathrm{J}$ and row-set $\mathrm{K}$ is the adjacency matrix of a cycle (see Nemhauser and Wolsey (1988)); 
(iii) at least three jobs in $J$ require $\mathrm{C}$ tools.

Then, the three tools mentioned under (iii) are heavy, and Theorem 6.3 applies again.

Consider now $\mathrm{p}$ subsets of tools $\mathrm{K}_{1}, \mathrm{~K}_{2}, \ldots, \mathrm{K}_{\mathrm{p}}$ for which we know (e.g., using Theorem 6.3) that at least one tool in $\mathrm{K}_{\mathrm{i}}$ incurs an extra setup in any sequence $(i=1,2, \ldots, p)$.

Let $\mathrm{K}=\cup_{\mathrm{i}} \mathrm{K}_{\mathrm{i}}$. Then, a lower bound on the total number of extra setups is provided by the optimal value of the following set covering problem :

$\mathrm{Z}=\min \quad \Sigma_{\mathrm{k} \in \mathrm{K}} \mathrm{t}_{\mathrm{k}}$

subject to

$$
\begin{array}{ll}
\sum_{\mathrm{k} \in \mathrm{K}_{\mathrm{i}} \mathrm{t}_{\mathrm{k}} \geq 1} & \mathrm{i}=1,2, \ldots, \mathrm{p} \\
\mathrm{t}_{\mathrm{k}} \in\{0,1\} & \mathrm{k} \in \mathrm{K} .
\end{array}
$$

Hence, $\mathrm{Z}+\mathrm{M}$ is a lower bound on $\operatorname{cost}(\mathrm{A}, \mathrm{C}$ ) (where $\mathrm{M}$ is, as always, the total number of tools).

\subsubsection{Valid inequalities}

Tang and Denardo (1988a) propose the following formulation of the tool switching problem (see also Bard (1988)). Let $\mathrm{x}_{\mathrm{ij}}=1$ if job $\mathrm{i}$ is the $\mathrm{j}$-th job in a sequence, and $x_{i j}=0$ otherwise; let $t_{k j}=1$ if tool $k$ is on the machine when the $\mathrm{j}$-th job is processed, and 0 otherwise; let $y_{k j}=1$ if tool $\mathbf{k}$ is setup just before processing the $\mathrm{j}$-th job of the sequence, and 0 otherwise $(k=1,2, \ldots, M ; i, j=1,2, \ldots, N)$. Then,

$$
\begin{array}{lll}
\operatorname{cost}(\mathrm{A}, \mathrm{C})=\min & \sum_{\mathrm{k}} \sum_{\mathrm{j}} \mathrm{y}_{\mathrm{kj}} & \\
\text { subject to } & \sum_{\mathrm{i}} \mathrm{x}_{\mathrm{ij}}=1 & \mathrm{j}=1,2, \ldots, \mathrm{N} \\
& \sum_{\mathrm{j}} \mathrm{x}_{\mathrm{ij}}=1 & \mathrm{i}=1,2, \ldots, \mathrm{N} \\
& \sum_{\mathrm{i}} \mathrm{a}_{\mathrm{ki}} \mathrm{x}_{\mathrm{ij}} \leq \mathrm{t}_{\mathrm{kj}} & \mathrm{k}=1,2, \ldots, \mathrm{M} ; \\
& \sum_{\mathrm{k}} \mathrm{t}_{\mathrm{kj}}=\mathrm{C}=1,2, \ldots, \mathrm{N} \\
& \mathrm{y}_{\mathrm{kj}} \geq \mathrm{t}_{\mathrm{kj}}-\mathrm{t}_{\mathrm{k}, \mathrm{j}-1} & \mathrm{j}=1,2, \ldots, \mathrm{N} \\
& \mathrm{k}=1,2, \ldots, \mathrm{M} ; \\
& \mathrm{j}=2, \ldots, \mathrm{N}
\end{array}
$$




$$
\begin{array}{ll}
y_{k 1} \geq t_{k 1} & k=1,2, \ldots, M \\
x_{i j} \in\{0,1\} & i, j=1,2, \ldots, N \\
t_{k j}, y_{k j} \in\{0,1\} & k=1,2, \ldots, M \\
& j=1,2, \ldots, N .
\end{array}
$$

Call $S W$ this $0-1$ programming problem. The linear programming relaxation of $S W$ provides a lower bound on cost (A, C). But this bound is extremely weak (Tang and Denardo (1988a)). One way of improving it would be to add more valid constraints to the formulation of SW. For instance, the following inequalities are valid for the tool switching problem :

$$
\sum_{\mathrm{j}} \mathrm{y}_{\mathrm{kj}} \geq 1 \quad \mathrm{k}=1,2, \ldots, \mathrm{M}
$$

(notice that these trivial inequalities are not even satisfied, in general, by an optimal solution to the continuous relaxation of SW). Another family of valid inequalities can be derived as follows. Let $\mathrm{K}$ be a subset of tools for which we know that at least one extra setup is required in the optimal sequence (see Theorem 6.3). Then,

$$
\sum_{\mathrm{k} \in \mathrm{K}} \quad \sum_{\mathrm{j}} \quad \mathrm{y}_{\mathrm{kj}} \geq|\mathrm{K}|+1
$$

is valid. More generally, if $\mathrm{Z}$ is the optimal value of $(6.2)-(6.4)$, then a valid constraint is given by :

$$
\Sigma_{k \in K} \quad \sum_{j} y_{k j} \geq|K|+Z .
$$

More work is needed on the strengthening of the formulation SW.

Still another possible approach would be to replace $S W$ by a formulation of the tool switching problem using different variables. For instance, one may want to consider the "disaggregated" variables $t_{k i j}$, with the interpretation that $\mathrm{t}_{\mathrm{kij}}=1$ if tool $\mathrm{k}$ is set up just after finishing the $\mathrm{i}$-th job and is removed just after finishing the $\mathrm{j}$-th job. It is easy to write a formulation of our problem involving only the variables $x_{i j}, t_{k i j}$ and $y_{k j}$. It is also relatively easy to derive exponentially many valid inequalities using these variables, which can in principle be added to the initial formulation in order to strengthen it. But our preliminary computational experiments along these lines were quite disappointing, in that they did not allow us to noticeably improve our previous lower bounds on the optimal value of the 
problem.

\subsubsection{Lagrangian relaxation}

Lagrangian relaxation is a classical tool in deriving bounds on the optimal value of an integer programming problem (see Nemhauser and Wolsey (1988)). For problem SW, one may for instance try to relax the groups of constraints (6.7) and (6.8). Indeed, as observed by Bard (1988), the resulting subproblems are then easy to solve (Bard '(1988) uses this relaxation scheme in order to produce a sequence of heuristic solutions for the tool switching problem). But it is easy to prove that the optimal value of the Lagrangian dual problem obtained in this way is equal to the optimal value of the linear relaxation of $S W$ (this is because all extreme points of the system defined by $(6.5),(6.6),(6.9),(6.10)$ and the relaxation of $(6.11),(6.12)$ are integral; see Nemhauser and Wolsey (1988)).

The possibility of deriving good lower bounds on cost $(\mathrm{A}, \mathrm{C})$ using Lagrangian relaxation is an avenue that should be further explored.

\subsection{Summary and conclusions}

In this chapter we analyze a problem occurring in certain flexible manufacturing environments. This problem was described by Tang and Denardo (1988a) and Bard (1988), and is here referred to as the tool switching problem. Links between this problem and other well-studied combinatorial optimization problems are established here for the first time: the matrix permutation problem (Möhring, 1990), optimization with greedy constraint matrices (Hoffman et al., 1985), the block minimization problem (Kou, 1977), recognition of interval matrices (Fulkerson and Gross, 1965), etc.

We prove that the tool switching is $N P$-hard, already for a fixed capacity $\mathrm{C}$ $\geq 2$. On the other hand, when the job sequence is fixed, we show that the problem of determining the optimal sequence of tool loadings can be modelled as a specially structured $0-1$ linear programming problem which can be solved in polynomial time. This provides an alternative proof of the correctness of the KTNS procedure.

In view of the complexity of the tool switching problem, several heuristic solution approaches are introduced. For instance, by modelling the tool switching problem as a shortest Hamiltonian path problem, well known 
heuristics for the latter problem become available. This was already noticed by Tang and Denardo (1988a), but the connection is here exploited in a systematic way by our traveling salesman and block minimization heuristics. Other new approaches include a greedy type heuristic and a heuristic based on the recognition of interval matrices. In addition, some improvement strategies are also proposed.

The performance of these heuristics is tested by applying them to a number of randomly generated problem instances. It turns out that the density of the tool job matrix affects the quality of the solutions obtained by some heuristics. In particular, the TSP-based heuristics of the type proposed by Tang and Denardo (1988a) perform poorly for sparse instances. The simple greedy heuristic performs well (in comparison with the other heuristics) on the sparse instances considered here, both in terms of quality of the solution found and of running time by the heuristic. The reason for this relatively good behaviour may be sought in the fact that greedy uses more information than e.g. the traveling salesman heuristics (see Section 6.3.3).

In order to formulate a more accurate judgement on the quality of the heuristics tested it would have been desirable to know tight and easily computed lower bounds on the cost of an optimal job sequence. The knowledge of such lower bounds would also be a prerequisite for the development of an exact optimization procedure (e.g. of the branch-andbound type) for the tool switching problem. Some of the research directions which may be worth pursuing in this regard are described. 


\section{Appendix}

\section{Graph-theoretic definitions}

In Chapter 6, a graph $\mathrm{G}$ is a triple of the form (V, E, d), where:

$\mathrm{V}$ is a finite set; the elements of $\mathrm{V}$ are the nodes of $\mathrm{G}$;

$\mathrm{E}$ is a set of pairs of nodes, called edges;

$\mathrm{d}$ is a function which assigns a nonnegative length to each pair of nodes; we assume that $d(u, v)=+\infty$ when $\{u, v\}$ is not an edge.

A path in a graph is a sequence of nodes, i.e. a permutation of a subset of $\mathrm{V}$. A traveling salesman path (or TS path) is a permutation of $\mathrm{V}$. The length of a path $\left(u_{1}, \ldots, u_{k}\right)$ is by definition:

$$
d\left(u_{1}, u_{2}\right)+d\left(u_{2}, u_{3}\right)+\ldots+d\left(u_{k-1}, u_{k}\right) .
$$

Notice, in particular, that the length of such a path is infinite if some pair $\left\{u_{i}, u_{i+1}\right\}$ is not an edge of the graph.

The traveling salesman problem on a graph $\mathrm{G}$ can be stated as follows: find a TS path of minimal length in G.

With a graph $\mathrm{G}=(\mathrm{V}, \mathrm{E}, \mathrm{d})$, we can associate another graph $\mathrm{H}=(\mathrm{E}, \mathrm{I}, \delta)$, called the edge-graph of $\mathrm{G}$, and defined as follows:

- each node of $\mathrm{H}$ is an edge of $\mathrm{G}$;

- a pair $\{e, f\}$, with e, $f \in E$, is an edge of $H$ if and only if the edges $e$ and $f$ share a common node in $G$;

- $\delta(e, f)=1$ if $\{e, f\}$ is an edge of $H$, and $\delta(e, f)=+\infty$ otherwise.

Observe that, in an edge-graph, every TS path has length either $|\mathrm{E}|-1$ or $+\infty$. Consider now the restriction of the traveling salesman problem to edge-graphs, that is: 


\section{Input: $\quad$ a graph $G$.}

Problem P3: $\quad$ find a TS path of minimal length in the edge-graph of $G$.

Equivalently, P3 asks whether there exists a TS path of finite length in the edge-graph of G. Bertossi (1981) proved that this problem is $N P$-hard.

We also deal in Chapter 6 with directed graphs. A directed graph is a triple $(V, U, d)$, where $V$ is defined as for a graph, and:

- $\quad \mathrm{U}$ is a set of ordered pairs of nodes, called arcs; i.e., $\mathrm{U} \subset \mathrm{VxV}$;

- $\quad d$ is a (nonnegative) length function defined on $V x V$, with the property that $d(u, v)=+\infty$ when $(u, v)$ is not an arc.

So, in a directed graph, $d(u, v)$ may differ from $d(v, u)$. The definitions of a TS path and of the TS problem extend in a straightforward way for directed graphs. 


\section{References}

Aanen, E. (1988), Planning and scheduling in a flexible manufacturing system, Ph.D. Thesis, University of Twente, Enschede.

Aggarwal, S.C. (1985), "MRP, JIT, OPT, FMS? Special report," Harvard Business Review 63 (5), 8-16.

Ahmadi, J., Grotzinger, S. and Johnson, D. (1988), "Component allocation and partitioning for a dual delivery placement machine," Operations Research 36 (2), 176-191.

Akella, R., Choong, Y. and Gershwin, S.B. (1984), "Performance of hierarchical production scheduling policy," in: Proceedings of the First ORSA/TIMS Special Interest Conference on Flexible Manufacturing Systems, Ann Arbor, MI, 385-396.

Ammons, J.C., Lofgren, C.B. and McGinnis, L.F. (1985), "A large scale machine loading problem in flexible assembly," Annals of Operations Research 3, 319-332.

Avonts, L.H. and Van Wassenhove, L.N. (1988), "The part mix and routing mix problem in FMS: a coupling between an LP model and a closed queueing network," International Joumal of Production Research 26 (12), 1891-1902.

Balas, E. and Ho, A. (1980), "Set covering algorithms using cutting planes, heuristics, and subgradient optimization: a computational study," Mathematical Programming Study 12, 37-60.

Ball, M.O. and Magazine, M.J. (1988), "Sequencing of insertions in printed circuit board assembly," Operations Research 36 (2), 192-201.

Bard, J.F. (1988), "A heuristic for minimizing the number of tool switches on a flexible machine," IIE Transactions 20 (4), 382-391. 
Bard, J.F. and Feo, T. (1989), "The cutting path and tool selection problem in computer aided process planning," Journal of Manufacturing Systems $8(1), 17-26$.

Bastos, J.M. (1988), "Batching and routing: two functions in the operational planning of flexible manufacturing systems," European Journal of Operational Research 33, 230-244.

Berrada, M. and Stecke, K.E. (1986), "A branch and bound approach for machine load balancing in flexible manufacturing systems," Management Science 32 (10), 1316-1335.

Bertossi, A.A. (1981), "The edge hamiltonian path problem is NP-complete," Information Processing Letters 13 (4,5), 157-159.

Blazewicz, J., Finke, G., Haupt, R. and Schmidt, G. (1988), "New trends in machine scheduling," European Journal of Operational Research 37, 303-317.

Booth, K.S. and Lueker, G.S. (1976), "Testing for the consecutive ones property, interval graph planarity using PQ-tree algorithms," Journal of Computer and System Sciences 13, 335-379.

Browne, J., Dubois, D., Rathmill, K., Sethi, S. and Stecke, K.E. (1984), "Classification of flexible manufacturing systems," The FMS Magazine $2(2), 114-117$.

Burkard, R.E. (1984), "Quadratic assignment problems," European Journal of Operational Research 15, 283-289.

Buzacott, J.A. and Yao, D.D. (1986), "Flexible manufacturing systems: a review of analytical models," Management Science 32 (7), 890-905.

Chaillou, P., Hansen, P. and Mahieu, Y. (1989), "Best network flow bounds for the quadratic knapsack problem," in: B. Simeone (ed.), Combinatorial Optimization, Springer-Verlag, Berlin, 225-235.

Chakravarty, A.K. and Shtub, A. (1984), "Selecting parts and loading flexible manufacturing systems," in: Proceedings of the First ORSA/TIMS Special Interest Conference on Flexible Manufacturing Systems, Ann Arbor, MI, 284-289.

Chams, M., Hertz, A. and de Werra, D. (1987), "Some experiments with simulated annealing for coloring graphs," European Joumal of Operational Research 32, 260-266.

Chang, Y.-L., Sullivan, R.S., Bagchi, U. and Wilson, J.R. (1985), "Experimental investigation of real-time scheduling in flexible 
manufacturing systems," Annals of Operations Research 3, 355-377.

Charles Stark Draper Laboratory (1984), Flexible Manufacturing Systems Handbook, Noyes Publications, Park Ridge, NJ.

Chung, C.H. (1991), "Planning tool requirements for flexible manufacturing systems," Journal of Manufacturing Systems 10 (6), 476-483.

Chvátal, V. (1983), Linear Programming, W.H. Freeman and Company, New York.

Cook, N.H. (1975), "Computer-managed parts manufacture," Scientific American 232, 22-28.

Cooper, R. and Jaikumar, R. (1984), "Management control of the flexible machining system," in: Proceedings of the First ORSA/TIMS Special Interest Conference on Flexible Manufacturing Systems, Ann Arbor, MI, 81-92.

CQM (1988), Philips Centre for Quantitative Methods, Eindhoven, The Netherlands.

Crama, Y., Kolen, A.W.J., Oerlemans, A.G. and Spieksma, F.C.R. (1989), "Throughput rate optimization in the automated assembly of printed circuit boards," Research Memorandum RM 89.034, Department of Quantitative Economics, University of Limburg, Maastricht.

Crama, Y. and Spieksma, F.C.R. (1992), "Approximation algorithms for three-dimensional assignment problems with triangle inequalities," European Journal of Operational Research 60 (3), to appear.

Dantzig, G.B. and Wolfe, P. (1960), "Decomposition principle for linear programs," Operations Research 8, 101-111.

Darrow, W.P. (1987), "An international comparison of flexible manufacturing systems technology," Interfaces 17 (6), 86-91.

Daskin, M., Jones, P.C. and Lowe, T.J. (1990), "Rationalizing tool selection in a flexible manufacturing system for sheet-metal products," Operations Research 38 (6), 1104-1115.

De Werra, D. and Widmer, M. (1990), "Loading problems with tool management in FMSs: a few integer programming models," The International Journal of Flexible Manufacturing Systems 3, 71-82.

Desrosiers, J., Soumis, F. and Desrochers, M. (1984), "Routing with time windows by column generation," Networks $14,545-565$. 
Dietrich, B.L., Lee, J. and Lee, Y.S. (1991), "Order selection on a single machine with high set-up costs," Working Paper OR 90-19, Yale University, New Haven.

Dorf, R.C. (1983), Robotics and Automated Manufacturing, Reston Publishing Company, Reston, VA.

Dupont-Gatelmand, C. (1982), "A survey of flexible manufacturing systems," Journal of Manufacturing Systems 1 (1), 1-16.

ElMaraghy, H.A. (1985), "Automated tooling management in flexible manufacturing," Journal of Manufacturing Systems 4 (1), 1-13.

Ettlie, J.E. (1988), "Implementation strategies for discrete parts manufacturing technologies," Final report, The University of Michigan, Ann Arbor, MI.

Farley, A.A. (1990), "A note on bounding a class of linear programming problems, including cutting stock problems," Operations Research 38 (5), 922-923.

Finke, G. and Kusiak, A. (1987), "Models for the process planning problem in a flexible manufacturing system," International Journal of Advanced Manufacturing Technology 2, 3-12.

Fisher, M.L. (1981), "The lagrangean relaxation method for solving integer programming problems," Management Science 27 (1), 1-18.

Fisher, M.L., Jaikumar, R. and Van Wassenhove, L.N. (1986), "A multiplier adjustment method for the generalized assignment problem," Management Science 32 (9), 1095-1103.

Fisk, J. and McKeown, P.G. (1979), "The pure fixed charge transportation problem," Naval Research Logistics 26, 631-641.

Förster, H.-U. and Hirt, K. (1989), "Entwicklung einer Handlungsanleitung zur Gestaltung von Produktionsplanungs- und -Steuerungskonzepten beim Einsatz flexibler Fertigungssysteme," Schlußbericht zum Forschungsvorhaben, $\mathrm{Nr}$. S 172, Forschungsinstitut für Rationalisierung, Rheinisch-Westfälische Technische Hochschule, Aachen.

Frieze, A.M. and Yadegar, J. (1981), "An algorithm for solving 3-dimensional assignment problems with application to scheduling a teaching practice," Journal of the Operational Research Society 32, 989-995. 
Fulkerson, D.R. and Gross, D.A. (1965), "Incidence matrices and interval graphs," Pacific Journal of Mathematics 15 (3), 833-835.

Gallo, G., Hammer, P.L. and Simeone, B. (1980), "Quadratic knapsack problems," Mathematical Programming Study 12, 132-149.

Gallo, G. and Simeone, B. (1988), "On the supermodular knapsack problem," Mathematical Programming 45, 295- 309.

Garey, M.R. and Johnson, D.S. (1979), Computers and Intractability: A Guide to the Theory of NP-Completeness, Freeman, New York.

Gerwin, D. (1982), "Do's and don'ts of computerized manufacturing," Harvard Business Review 60 (2), 107-116.

Gilmore, P.C. and Gomory, R.E. (1961), "A linear programming approach to the cutting-stock problem," Operations Research 9, 849-859.

Glover, F. (1989), "Tabu search - part I," ORSA Journal on Computing 1 (3), 190-206.

Glover, F. (1990), "Tabu search - part II," ORSA Journal on Computing 2 (1), 4-32.

Golden, B.L. and Stewart, W.R. (1985), "Empirical analysis of heuristics," in: E.L. Lawler, J.K. Lenstra, A.H.G. Rinnooy Kan and D.B. Shmoys (eds.), The Traveling Salesman Problem, John Wiley \& Sons, Chichester, 207-249.

Goldhar, J.D. and Jelinek, M. (1985), "Computer integrated flexible manufacturing: organizational, economic, and strategic implications," Interfaces 15 (3), 94-105.

Graver, T.W. and McGinnis, L.F. (1989), "A tool provisioning problem in an FMS," The Intemational Joumal of Flexible Manufacturing Systems $1,239-254$.

Graves, S.C. and Lamar, B.W. (1983), "An integer programming procedure for assembly system design problems," Operations Research 31 (3), 522-545.

Gray, A.E., Seidmann, A. and Stecke, K.E. (1988), "Tool management in automated manufacturing: operational issues and decision problems," Working Paper CMOM 88-03, Simon Graduate School of Business Administration, University of Rochester, New York.

Greene, T.J. and Sadowski, R.P. (1986), "A mixed integer program for loading and scheduling multiple flexible manufacturing cells," European 
Joumal of Operational Research 24, 379-386.

Groover, M.P. (1980), Automation, Production Systems, and Computer-Aided Manufacturing, Prentice-Hall, Englewood Cliffs, NJ.

Groover, M.P. and Zimmers jr., E.W. (1984), CAD/CAM Computer-Aided Design and Manufacturing, Prentice-Hall, Englewood Cliffs, NJ.

Gruver, W.A. and Senninger, M.T. (1990), "Tooling management in FMS," Mechanical Engineering 112 (3), 40-44.

Gupta, Y.P. and Goyal, S. (1989), "Flexibility of manufacturing systems: concepts and measurements," European Journal of Operational Research 43, 119-135.

Hartley, J. (1984), FMS at Work, IFS/North-Holland, Amsterdam.

Hirabayashi, R., Suzuki, H. and Tsuchiya, N. (1984), "Optimal tool module design problem for NC machine tools," Journal of the Operations Research Society of Japan 27 (3), 205-228.

Hoffman, A.J., Kolen, A.W.J. and Sakarovitch, M. (1985), "Totally balanced and greedy matrices," SIAM Journal on Algebraic and Discrete Methods 6 (4), 721-730.

Holstein, W.K. (1968), "Production planning and control integrated," Harvard Business Review 46 (3), 121-140.

Huang, P.Y. and Chen, C. (1986), "Flexible manufacturing systems: an overview and bibliography," Production and Inventory Management Third Quarter, 80-90.

Hwang, S. (1986), "A constraint-directed method to solve the part selection problem in flexible manufacturing systems planning stage," in: K.E. Stecke and R. Suri (eds.), Proceedings of the Second ORSA/TIMS Conference on Flexible Manufacturing Systems, Elsevier Science Publishers B.V., Amsterdam, 297-309.

Hwang, S.S. and Shogan, A.W. (1989), "Modelling and solving an FMS part selection problem," International Joumal of Production Research 27 (8), 1349-1366.

Hyer, N. and Wemmerlöv, V. (1984), "Group technology and productivity," Harvard Business Review 62 (4),

Jaikumar, R. (1986), "Postindustrial manufacturing," Harvard Business Review 64 (6), 69-76. 
Jaikumar, R. and Van Wassenhove, L.N. (1989), "A production planning framework for flexible manufacturing systems," Journal of Manufacturing and Operations Management 2, 52-79.

Jain, A.K., Kasilingam, G. and Bhole, S.D. (1991), "Joint consideration of cell formation and tool provisioning problems in flexible manufacturing systems," Computers \& Industrial Engineering 20 (2), 271-277.

Jaumard, B., Hansen, P. and Poggi de Aragāo, M. (1991), "Column generation methods for probabilistic logic," ORSA Journal on Computing 3 (2), 135-148.

Johnson, D.S., Aragon, C.R., McGeoch, L.A. and Schevon, C. (1989), "Optimization by simulated annealing: an experimental evaluation; part I, graph partitioning," Operations Research 37 (6), 865-892.

Johnson, D.S., Aragon, C.R., McGeoch, L.A. and Schevon, C. (1991), "Optimization by simulated annealing: an expirimental evaluation; part II, graph coloring and number partitioning," Operations Research 39 (3), $378-406$.

Johnson, D.S. and Papadimitriou, C.H. (1985), "Computational complexity," in: E.L. Lawler, J.K. Lenstra, A.H.G. Rinnooy Kan and D.B. Shmoys (eds.), The Traveling Salesman Problem, John Wiley \& Sons, Chichester, 37-85.

Kaplan, R.S. (1986), "Must CIM be justified by faith alone?," Harvard Business Review 64 (2), 87-95.

Kashiwabara, T. and Fujisawa, T. (1979), "NP-completeness of the problem of finding a minimum-clique-number interval graph containing a given graph as a subgraph," in: Proc. 1979 Intern. Symposium on Circuits and Systems, 657-660.

Kavvadias, D. and Papadimitriou, C.H. (1989), "A linear programming approach to reasoning about probabilities," Annals of Mathematics and Artificial Intelligence 1, 189-205.

Kernighan, B.W. and Lin, S. (1970), "An efficient heuristic procedure for partitioning graphs," The Bell System Technical Journal 49 (2), 291-307.

Kim, Y.D. and Yano, C.A. (1992), "An iterative approach to system setup problems in flexible manufacturing systems," The International Journal of Flexible Manufacturing Systems 4, 183-209.

Kiran, A.S. and Krason, R.J. (1988), "Automated tooling in a flexible manufacturing system," Industrial Engineering April, 52-57. 
Kiran, A.S. and Tansel, B.C. (1986), "The system setup in FMS: concepts and formulation," in: K.E. Stecke and R. Suri (eds.), Proceedings of the Second ORSA/TIMS Conference on Flexible Manufacturing Systems, Elsevier Science Publishers B.V., Amsterdam, 321- 332.

Korte, B. (1989), "Applications of combinatorial optimization," in: M. Iri and K. Tanabe (eds.), Mathematical Programming, Recent Developments and Applications, KTK Scientific Publ., Tokyo, 1-55.

Kou, L.T. (1977), "Polynomial complete consecutive information retrieval problems," SIAM Journal on Computing 6, 67-75.

Kuhn, H. (1990), Einlastungsplanung von flexiblen Fertigungssystemen, Physica-Verlag, Heidelberg.

Kumar, K.R., Kusiak, A. and Vanelli, A. (1986), "Grouping of parts and components in flexible manufacturing systems," European Journal of Operational Research 24, 387-397.

Kusiak, A. (1985a), "Flexible manufacturing systems: a structural approach," International Journal of Production Research 23 (6), 1057-1073.

Kusiak, A. (1985b), "Integer programming approach to process planning," International Journal of Advanced Manufacturing Technology 1 (1), 73-83.

Kusiak, A. (1985c), "The part families problem in flexible manufacturing systems," Annals of Operations Research 3, 279-300.

Kusiak, A. (1986), "Application of operational research models and techniques in flexible manufacturing systems," European Journal of Operational Research 24, 336-345.

Lawler, E.L., Lenstra, J.K., Rinnooy Kan, A.H.G. and Shmoys, D.B. (1985), The Traveling Salesman Problem, John Wiley \& Sons, Chichester.

Leipälä, T. and Nevalainen, O. (1989), "Optimization of the movements of a component placement machine," European Journal of Operational Research 38, 167-177.

Mamer, J.W. and Shogan, A.W. (1987), "A constrained capital budgetting problem with applications to repair kit selection," Management Science 33 (6), 800-806.

Mattson, R., Gecsei, J., Slutz, D.R. and Traiger, I.L. (1970), "Evaluation techniques for storage hierarchies," IBM Systems Journal 9 (2), 78-117. 
Mazzola, J.B., Neebe, A.W. and Dunn, C.V.R. (1989), "Production, planning of a flexible manufacturing system in a material requirements planning environment," The International Journal of Flexible Manufacturing Systems 1 (2), 115-142.

Meredith, J.R. (1987), "Implementing new manufacturing technologies: managerial lessons over the FMS life cycle," Interfaces 17 (6), 51-62.

Minoux, M. (1987), "A class of combinatorial problems with polynomially solvable large scale set covering/partitioning relaxations," R.A.I.R.O. Recherche opérationnelle/Operations Research 21 (2), 105-136.

Möhring, R.H. (1990), "Graph problems related to gate matrix layout and PLA folding," in: G. Tinhofer et al. (eds.), Computational Graph Theory, Springer-Verlag, Wien, 17-51.

Monahan, G.E. and Smunt, T.L. (1987), "A multilevel decision support system for the financial justification of automated flexible manufacturing systems," Interfaces 17 (6), 29-40.

Montazeri, M. and Van Wassenhove, L.N. (1990), "Analysis of scheduling rules for an FMS," International Journal of Production Research 28 (4), 785-802.

Mullins, P. (1990), "PCB assembly: a total package," Production 102 (2), 60-61.

Nemhauser, G.L. and Wolsey, L.A. (1988), Integer and Combinatorial Optimization, John Wiley \& Sons, New York.

Panwalker, S.S. and Iskander, W. (1977), "A survey of scheduling rules," Operations Research 25 (1), 45-61.

Papadimitriou, C.H. and Steiglitz, K. (1982), Combinatorial Optimization, Algorithms and Complexity, Prentice Hall, Inc., Englewood Cliffs, NJ.

Primrose, P.L. and Leonard, R. (1991), "Selecting technology for investment in flexible manufacturing," The International Journal of Flexible Manufacturing Systems 4 (1), 51-77.

Rajagopalan, S. (1985), "Scheduling problems in flexible manufacturing systems," Working Paper, Graduate School of Industrial Administration, Carnegie-Mellon University, Pittsburgh, PA.

Rajagopalan, S. (1986), "Formulation and heuristic solutions for parts grouping and tool loading in flexible manufacturing systems," in: K.E. Stecke and R. Suri (eds.), Proceedings of the Second ORSA/TIMS Conference on Flexible Manufacturing Systems, Elsevier Science 
Publishers B.V., Amsterdam, 311-320.

Ránky, P.G. (1983), The Design and Operation of FMS, IFS/North-Holland, Amsterdam.

Ranta, J. and Tchijov, I. (1990), "Economics and success factors of flexible manufacturing systems: the conventional explanation revisited," The International Joumal of Flexible Manufacturing Systems 2, 169-190.

Ribeiro, C.C., Minoux, M. and Penna, M.C. (1989), "An optimal column-generation-with-ranking algorithm for very large scale set partitioning problems in traffic assignment," European Joumal of Operational Research 41, 232-239.

Roger, C. (1990), "La gestion des outils sur machines à commande numérique," Mémoire DEA de Recherche Opérationnelle, Mathématiques et Informatique de la Production, Université Joseph Fourier, Grenoble.

Sethi, A.K. and Sethi, S.P. (1990), "Flexibility in manufacturing: a survey," The International Joumal of Flexible Manufacturing Systems 2, 289-328.

Shanker, K. and Tzen, Y.J. (1985), "A loading and dispatching problem in a random flexible manufacturing system," International Joumal of Production Research 23 (3), 579-595.

Singhal, K., Fine, C.H., Meredith, J.R. and Suri, R. (1987), "Research and models for automated manufacturing," Interfaces 17 (6), 5-14.

Solot, P. and van Vliet, M. (1990), "Analytical models for FMS design optimization: a survey," Working Paper ORWP 90/16, Ecole Polytechnique Fédérale de Lausanne, Lausanne.

Spieksma, F.C.R. (1992), Assignment and scheduling algorithms in automated manufacturing, Ph.D. Thesis, University of Limburg, Maastricht.

Spieksma, F.C.R., Oerlemans, A.G. and Vrieze, K. (1990), "On the system setup and the scheduling problem in a flexible manufacturing system," Statistica Neerlandica 44 (3), 125-138.

Stecke, K.E. (1983), "Formulation and solution of nonlinear integer production planning problems for flexible manufacturing systems," Management Science 29, 273-288.

Stecke, K.E. (1985), "Design, planning, scheduling, and control problems of flexible manufacturing systems," Annals of Operations Research 3, $3-12$. 
Stecke, K.E. (1988), “O.R. applications to flexible manufacturing," in: G.K. Rand (ed.), Operational Research '87, 217-232.

Stecke, K.E. (1989), "Algorithms for efficient planning and operation of a particular FMS," The International Journal of Flexible Manufacturing Systems 1 (4), 287-324.

Stecke, K.E. and Browne, J. (1985), "Variations in flexible manufacturing systems according to the relevant types of automated materials handling," Material Flow 2, 179-185.

Stecke, K.E. and Kim, I. (1988), "A study of FMS part type selection approaches for short-term production planning," The International Journal of Flexible Manufacturing Systems 1 (1), 7-29.

Stecke, K.E. and Solberg, J.J. (1981), "Loading and control policies for a flexible manufacturing system," Intemational Journal of Production Research 19 (5), 481-490.

Suri, R. (1985), "An overview of evaluative models for flexible manufacturing systems," Annals of Operations Research 3, 13-21.

Suri, R. and Whitney, C.K. (1984), "Decision support requirements in flexible manufacturing," Joumal of Manufacturing Systems 3 (1), 61-69.

Tang, C.S. and Denardo, E.V. (1988a), "Models arising from a flexible manufacturing machine, part I: minimization of the number of tool switches," Operations Research 36 (5), 767-777.

Tang, C.S. and Denardo, E.V. (1988b), "Models arising from a flexible manufacturing machine, part II: minimization of the number of switching instants," Operations Research 36 (5), 778-784.

Van Laarhoven, P.J.M. and Aarts, E.H.L. (1987), Simulated Annealing: Theory and Applications, D. Reidel Publishing Company, Dordrecht.

Van Laarhoven, P.J.M. and Zijm, W.H.M. (1993), "Production preparation and numerical control in PCB assembly," The International Journal of Flexible Manufacturing Systems 5 (3), to appear.

Van Looveren, A.J., Gelders, L.F. and Van Wassenhove, L.N. (1986), "A review of FMS planning models," in: A. Kusiak (eds.), Modelling and Design of Flexible Manufacturing Systems, Elsevier Science Publishers B.V., Amsterdam, 3-31.

Van Vliet, M. and Van Wassenhove, L.N. (1989), "Operational research techniques for analyzing flexible manufacturing systems," Research 
Memorandum Series, No. TI-1989/16, Tinbergen Institute, Erasmus University Rotterdam, Rotterdam.

Vasko, F.J. and Wolf, F.E. (1988), "Solving large set covering problems on a personal computer," Computers \& Operations Research 15 (2), 115-121.

Ventura, J.A., Chen, F.F. and Leonard, M.S. (1988), "Loading tools to machines in flexible manufacturing systems," Computers \& Industrial Engineering 15 (1-4), 223-230.

Volgenant, T. and Jonker, R. (1982), "A branch and bound algorithm for the symmetric traveling salesman problem based on l-tree relaxation," European Joumal of Operational Research 9, 83-89.

Warnecke, H.-J. and Steinhilper, R. (1985), Flexible Manufacturing Systems, IFS Publications, Springer-Verlag, Berlin.

Whitney, C.K. and Gaul, T.S. (1985), "Sequential decision procedures for batching and balancing in FMSs," Annals of Operations Research 3, 301-316.

Whitney, C.K. and Suri, R. (1985), "Algorithms for part and machine selection in flexible manufacturing systems," Annals of Operations Research 3, 239-261.

Widmer, M. (1991), "Job shop scheduling with tooling constraints: a tabu search approach," Journal of the Operational Research Society 42 (1), $75-82$.

Zeestraten, M.J. (1989), Scheduling flexible manufacturing systems, Ph.D. Thesis, Delft University of Technology, Delft.

Zijm, W.H.M. (1988), "Flexible manufacturing systems: background, examples and models," in: H. Schellhaas et al. (eds.), Operations Research Proceedings 1988, Springer-Verlag, Heidelberg, 142-161. 


\section{Author index}

Aanen,E. 11

Aarts, E.H.L. 113

Aggarwal, S.C. 2

Ahmadi, J. 21, 26, 29, 34

Akella, R. 16

Ammons, J.C. 14

Aragon, C.R. 110, 113

Avonts, L.H. 12

Bagchi, U. 16

Balas, E. 52

Ball, M.O. 21, 25, 39

Bard, J.F. 15, 19, 50, 125 , $126,131,139$, $140,142,151$, 153

Bastos, J.M. 16

Berrada, M. 10, 13

Bertossi, A.A. 156

Bhole, S.D. 15

Blazewicz, J. 16, 125

Booth, K.S. 138, 139

Browne, J. 5, 6

Burkard, R.E. 43, 44

Buzacott, J.A. 4, 9-11, 22

Chaillou, P. 56

Chakravarty, A.K. 13, 50

Chams, M. 110

Chang, Y.-L. 16

Charles Stark Draper Laboratory 4
Chen, C. 3, 4

Chen, F.F. 15

Choong, Y. 16

Chung, C.H. 15

Chvátal, V. 53, 54

Cook, N.H. 2

Cooper, R. 12

CQM 25, 40, 44, 45, 48

Crama, Y. 29, 38, 39

Dantzig, G.B. 53

Darrow, W.P. 7

Daskin, M. 15, 131

De Werra, D. 15, 110

Denardo, E.V. 15, 17, 19 , $50,51,62,63$, $68,69,73,77$, $78,85,86,96$, $98,99,125,126$, $129,131,135$, $141,142,149$, 151-154

Desrochers, M. 54

Desrosiers, J. 54

Dietrich, B.L. 56, 64, 66

Dorf, R.C. 3

Dubois, D. 5

Dunn, C.V.R. 82

Dupont-Gatelmand, C. 4

ElMaraghy, H.A. 15

Ettlie, J.E. 6 
Farley, A.A. 57, 58, 85

Feo, T. 50, 125

Fine, C.H. 9, 10

Finke, G. 16, 50, 125, 128, 131,140

Fisher, M.L. 61

Fisk, J. 32

Förster, H.-U. 125, 143

Frieze, A.M. 38, 39

Fujisawa, T. 129

Fulkerson, D.R. 138, 139, 153

Gallo, G. 55, 56

Garey, M.R. 29, 38

Gaul, T.S. $13,14,17,50$, 64,86

Gecsei, J. 131

Gelders, L.F. 9

Gershwin, S.B. 16

Gerwin, D. 3

Gilmore, P.C. 53, 54

Glover, F. 112, 113

Golden, B.L. 134, 135

Goldhar, J.D. 9

Gomory, R.E. 53, 54

Goyal, S. 5

Graver, T.W. 15

Graves, S.C. 12

Gray, A.E. $15,49,125$

Greene, T.J. 16

Groover, M.P. 2, 4

Gross, D.A. 138, 139, 153

Grotzinger, S. 21

Gruver, W.A. 15

Gupta, Y.P. 5

Hammer, P.L. 55

Hansen, P. 55, 56

Hartley, J. 4, 9

Haupt, R. 16, 125

Hertz, A. 110

Hirabayashi, R. 15, 17, 50, $51,55,84,125$
Hirt, K. 125, 143

Ho, A. 52

Hoffman, A.J. 131, 133, 134,153

Holstein, W.K. 9

Huang, P.Y. 3, 4

Hwang, S.S. 14, 17, 50, 51, $55,69,81,97$, 105

Hyer, N. 12

Iskander, W. 16

Jaikumar, R. 3, 6, 9, 11, 12,

Jain, A.K. 15

$$
14,61,97
$$

Jaumard, B. 55

Jelinek, M. 9

Johnson, D. 21

Johnson, D.S. 29, 38, 110, $113,114,135$

Jones, P.C. 15, 131

Jonker, R. 136

Kaplan, R.S. 7, 8

Kashiwabara, T. 129

Kasilingam, G. 15

Kavvadias, D. 55

Kernighan, B.W. 108, 112, 114

Kim, I. 14, 50

Kim, Y.D. 15

Kiran, A.S. 10, 14, 15, 49, 125

Kolen, A.W.J. 29, 131

Korte, B. 22

Kou, L.T. 137, 153

Krason, R.J. 15, 49, 125

Kuhn, H. 50, 69

Kumar, K.R. 13

Kusiak, A. 9-13, 50, 81, 125, 128

Lamar, B.W. 12

Lawler, E.L. 40, 41

Lee, J. 56 
Lee, Y.S. 56

Leipälä, T. 21, 26, 39-41, 43

Lenstra, J.K. 40

Leonard, M.S. 15

Leonard, R. 7, 8

Lin, S. 108, 112, 114

Lofgren, C.B. 14

Lowe, T.J. 15, 131

Lueker, G.S. 138, 139

Magazine, M.J. 21, 25, 39

Mahieu, Y. 56

Mamer, J.W. 56

Mattson, R. 131

Mazzola, J.B. 82, 88

McGeoch, L.A. 110, 113

McGinnis, L.F. 14, 15

Mckeown, P.G. 32

Meredith, J.R. 9, 10

Minoux, M. 54

Möhring, R.H. 129, 153

Monahan, G.E. 12

Montazeri, M. 16

Mullins, P. 17

Neebe, A.W 82

Nemhauser, G.L. 29, 32, 52, $62,65,87,129$, $131,134,135$, $138,150,153$

Nevalainen, O. 21, 26, 39$-41,43$

Oerlemans, A.G. 16, 29

Panwalker, S.S. 16

Papadimitriou, C.H. 38, 55, 135

Penna, M.C. 54

Poggi de Aragão, M. 55

Primrose, P.L. 7, 8

Rajagopalan, S. 14, 17, 50, $64,69,81,82$, $86,88,96,99$, 105

Ránky, P.G. 4
Ranta, J. 7-9

Rathmill, K. 5

Ribeiro, C.C. 54

Rinnooy Kan, A.H.G. 40

Roger, C. 131, 140

Sadowski, R.P. 16

Sakarovitch, M. 131

Schevon, C. 110,113

Schmidt, G. 16, 125

Seidmann, A. 15, 125

Senninger, M.T. 15

Sethi, A.K. 5

Sethi, S.P. 5

Shanker, K. 16, 82, 88

Shmoys, D.B. 40

Shogan, A.W. 14, 50, 55, $56,69,97,105$

Shtub, A. 13, 50

Simeone, B. 55, 56

Singhal, K. 9, 10

Slutz, D.R. 131

Smunt, T.L. 12

Solberg, J.J. 10, 13, 16

Solot, P. 12

Soumis, F. 54

Spieksma, F.C.R. 16, 17, 29, 38,39

Stecke, K.E. 3, 5, 6, 9-11, $13-16,50,81,82$, $88,90,96,125$

Steiglitz, K. 38

Steinhilper, R. 4

Stewart, W.R. 134, 135

Sullivan, R.S. 16

Suri, R. 9-12

Suzuki, H. 15, 50, 84, 125

Tang, C.S. 15, 17, 19, 50, $51,62,63,68$, $69,73,77,78$, $85,86,96,98$, $99,125,126$, 129, 131, 135, 


$$
\begin{aligned}
& 141,142, \\
& 149,151- \\
& -154
\end{aligned}
$$

Tansel, B.C. 10,14

Tchijov, I. 7-9

Traiger, I.L. 131

Tsuchiya, N. $15,50,84,125$

Tzen, Y.L. 16, 82, 88

Van Laarhoven, P.J.M. 17,

$$
25,113
$$

Van Looveren, A.J. 9-12, 15

Van Vliet, M. 11, 12

Van Wassenhove, L.N. 6, 9,

$$
\begin{aligned}
& 11,12,14,16, \\
& 61,97
\end{aligned}
$$

Vanelli, A. 13

Vasko, F.J. 52

Ventura, J.A. 15

Volgenant, T. 136

Vrieze, K. 16

Warnecke, H.-J. 4

Wemmerlöv, V. 12

Whitney, C.K. 9, 10, 12-14,

$$
17,50,64,86
$$

Widmer, M. 15

Wilson, J.R. 16

Wolf, F.E. 52

Wolfe, P. 53

Wolsey, L.A. 29, 32, 52, 62, $65,87,129,131$, $134,135,138$, 150,153

Yadegar, J. 38, 39

Yano, C.A. 15

Yao, D.D. 4, 9-11, 22

Zeestraten, M:J. 11, 16

Zijm, W.H.M. 2, 11, 12, 16,

$$
17,25
$$

Zimmers jr., E.W. 2 


\section{Samenvatting}

\section{Produktieplanning voor flexibele produktiesystemen}

Produktieplanning is essentieel voor het efficiënt gebruik van moderne produktiesystemen. In dit proefschrift worden methoden ontwikkeld, die kunnen worden toegepast om een aantal specifieke produktieplanningsproblemen in flexibele produktiesystemen (FPS) op te lossen. Eerst zal worden ingegaan op de flexibele produktietechnologie en vervolgens zal per hoofdstuk een overzicht worden gegeven van het onderzoek.

Het industriële landschap bestaat uit een wijd spectrum van produktiefaciliteiten. Aan de ene kant van het spectrum is er sprake van stuksproduktie, waar met behulp van machines kleine series van een bepaald produkt worden gemaakt. De machines in een dergelijk systeem kunnen verschillende produkten fabriceren. Wanneer er wordt overgeschakeld op een ander type produkt worden de machines omgesteld, hetgeen echter wel produktieverlies betekent. Het voordeel van het maken van kleine series of individuele produkten is de flexibiliteit, die aanwezig is om tegemoet te komen aan de specifieke wensen uit de markt. Aan de andere kant van het spectrum is er sprake van massaproduktie. De machines zijn gebouwd voor een bepaald produkt of een klasse van produkten. Overgaan op een ander produkt is erg kostbaar omdat de machines mogelijk geheel moeten worden aangepast. Het grote voordeel is het grote produktievolume, waardoor de kosten per eenheid voor één type produkt relatief laag zijn. Massaproduktie kenmerkt zich door een starheid als het gaat om het snel en goedkoop reageren op een veranderende vraag. Flexibele produktiesystemen (FPS) zijn ontworpen om de voordelen van beide systemen te combineren. Dit gebeurt door de omsteltijden, die nodig zijn om van één produkt over te schakelen op een ander produkt aanzienlijk te verminderen. In plaats van machines, die slechts 
één specifieke functie kunnen vervullen, worden machines ingezet, die een magazijn hebben, waarin verschillende gereedschappen kunnen worden opgeslagen. Gereedschappen, die aanwezig zijn in het magazijn, kunnen automatisch in enkele seconden worden verwisseld door een gereedschappenverwisselaar, die aan iedere machine is bevestigd. Hierdoor is het mogelijk dat snel en zonder noemenswaardig produktieverlies kan worden overgeschakeld van het ene naar het andere produkt. De omsteltijden bij stuksproduktie worden op deze wijze verminderd, terwijl tevens de starheid, die massaproduktiesystemen kenmerkt, wordt voorkomen.

Een FPS bestaat uit een aantal machines met een gereedschappenmagazijn, een transportsysteem, een aantal voorraadbuffers, aanvoer- en afvoerstations, eventueel afwerkingsmachines en een computersysteem, dat zorgt voor de coördinatie van de diverse functies en sturing van de apparatuur. Het transport kan plaatsvinden via een transportband, die de machines, de aanvoer- en afvoerplaatsen en buffers met elkaar verbindt, maar er wordt ook in toenemende mate gebruik gemaakt van automatisch gestuurde wagens, die langs de diverse lokaties in het systeem worden geleid.

De eerste FPS zijn aan het eind van de jaren '60 ontworpen. Momenteel zijn wereldwijd 500-1500 systemen in gebruik (het exacte aantal is afhankelijk van de aangehangen definitie voor FPS). Een FPS is technologisch complex en de investeringen in het systeem en de opleiding van het personeel zijn hoog. De systemen zijn het meest geschikt in omgevingen, waar het gewenst is om geregeld de machines om te stellen. De belangrijkste toepassingen van FPS zijn te vinden in de metaalindustrie (de produktie van specifieke motor- en machineonderdelen), maar ook in de elektronica zijn er toepassingen, onder andere in de fabricage van printplaten, die te vinden zijn in tal van industriële en consumentenprodukten. Momenteel bevindt FPS-technologie zich niet meer in een test-fase, maar heeft zij zich ruimschoots bewezen in tal van toepassingen.

Dit proefschrift richt zich op een aantal problemen binnen de produktieplanning van FPS. De complexiteit van de produktieplanningsproblemen nodigt uit tot het splitsen van deze problemen in een aantal deelproblemen, die beter hanteerbaar zijn. Nadeel van deze methode is het risico dat het oplossen van de deelproblemen niet leidt tot een oplossing van het totale probleem in verband met het weglaten van interacties tussen de deelproblemen. Het voordeel is echter dat door gebruik te maken van een opzet met hiërarchisch geordende deelproblemen een beter inzicht kan worden verkregen in de fundamentele problemen, die ten grondslag liggen aan het produktieplanningsprobleem. In hoofdstuk 2 van dit proefschrift wordt een hiërarchische oplossingsmethode voorgesteld voor een produktieplanningsprobleem in de elektronica-sector. In de overige hoofdstukken van het proefschrift worden een aantal produktieplanningsproblemen besproken, die betrekking hebben op 
toewijzingsproblemen van opdrachten en gereedschappen aan machines.

In hoofdstuk 2 wordt een produktieplanningsprobleem besproken uit de praktijk. Het probleem betreft het maximaliseren van de produktiesnelheid bij de produktie van printplaten (printed circuit boards). Printplaten worden algemeen toegepast in de consumentenelectronika (onder andere computers, audio en video) en de industrie (bijvoorbeeld in telecommunicatie). De produktie van printplaten vindt hoofdzakelijk plaats met behulp van computergestuurde $(\mathrm{CNC})$ machines en de technologie verandert snel. Er wordt een probleem bestudeerd, waarbij een vastgesteld aantal componenten op een printplaat moet worden bevestigd met behulp van een aantal machines. Er moet worden vastgesteld welke componenten door welke machine moeten worden bevestigd. Tevens moet worden bepaald op welke wijze en in welke volgorde de componenten door de machines moeten worden bevestigd. Dit produktieplanningsprobleem wordt aangepakt met behulp van een hiërarchische oplossingsmethode, waarbij het probleem wordt gesplitst in een aantal deelproblemen, die apart moeten worden opgelost. Een aantal van deze deelproblemen blijkt $N P$-moeilijk te zijn. Dit betekent dat het oplossen van deze problemen met gangbare methoden exponentieel veel rekentijd vraagt. Het optimaal oplossen van grotere $N P$-moeilijke problemen duurt hierdoor extreem lang. In dit soort gevallen wordt daarom gebruik gemaakt van heuristische methoden, hetgeen ook in dit onderzoek is gebeurd. De hiërarchische oplossingsmethode is met succes getest op een praktijkgeval, waarbij 258 componenten van 39 verschillende typen op een printplaat moeten worden bevestigd met behulp van een lijn met drie machines. De geschatte reductie in produktietijd bedroeg tenminste 18 procent.

In hoofdstuk 3 wordt een tool management probleem besproken. Tool management, het efficiënt plannen van het gebruik van de gereedschappen, die door de machines van het FPS worden gebruikt, is essentieel voor het efficiënt gebruik maken van het systeem. In conventionele produktiesystemen ligt de nadruk op de planning van de opdrachten, waarbij vastligt welke opdrachten door bepaalde machines kunnen worden vervuld. In FPS kunnen machines diverse opdrachten vervullen, afhankelijk van de gereedschappen, die aanwezig zijn in de magazijnen van de machines. Deze hebben een beperkte capaciteit variërend van 10 tot 250 (maar doorgaans enkele tientallen) gereedschappen. In de planningsfase moet worden bepaald welke gereedschappen in het magazijn worden bewaard, waarmee de mogelijke functies van de machines worden vastgelegd. Indien een opdracht moet worden uitgevoerd met een gereedschap, dat niet aanwezig is in het magazijn van de machine, moet de samenstelling van het magazijn worden aangepast. In dit geval is een langere omsteltijd noodzakelijk. Efficiënt tool management richt zich op het efficiënt inrichten van de magazijnen, zodat omsteltijden zoveel mogelijk worden vermeden. In hoofdstuk 3 wordt een model 
gepresenteerd dat zich richt op het minimaliseren van het aantal keren dat een machine moet worden omgesteld ten gevolge van een toevoeging van gereedschappen aan het magazijn. We veronderstellen dat een aantal opdrachten moeten worden uitgevoerd op een machine. Voor iedere opdracht zijn een aantal gereedschappen nodig, die aanwezig dienen te zijn in het magazijn bij het uitvoeren van de opdracht. We noemen een groep van opdrachten uitvoerbaar, indien de gereedschappen, die nodig zijn voor deze opdrachten tegelijk in het magazijn van de machine passen. Voor een uitvoerbare groep hoeft de machine dus maar één keer te worden ingesteld. Het job grouping probleem bestaat uit het splitsen van de opdrachten in een minimaal aantal uitvoerbare groepen. Het job grouping probleem behoort tot de klasse van $N P$-moeilijke problemen, waardoor het exact oplossen van het probleem wordt bemoeilijkt.

Diverse auteurs hebben methoden voorgesteld, die een benadering geven voor het minimaal aantal uitvoerbare groepen (zie hoofdstuk 3). De kwaliteit van deze methoden werd vastgesteld door de resultaten van de methoden te vergelijken met de optimale oplossing, die kan worden verkregen door (zeer lange) computerberekeningen met behulp van branch-and-bound methoden. Wij maken gebruik van een set covering formulering om een ondergrens te vinden voor het aantal uitvoerbare groepen. Het oplossen van de lineaire relaxatie van deze formulering geeft een ondergrens, die nauw aansluit bij de optimale waarde. Hierdoor is het exact berekenen van deze waarde niet meer noodzakelijk. De lineaire relaxatie wordt berekend met behulp van een kolom generatie methode. Tevens worden enkele methoden voorgesteld voor het vinden van oplossingen voor het probleem. De resultaten worden bevestigd door computerexperimenten op een grote verzameling van testproblemen. Uit deze berekeningen blijkt dat de kwaliteit van de ondergrens en van een aantal heuristische methoden goed is.

In hoofdstuk 4 worden de resultaten van hoofdstuk 3 uitgebreid naar enkele algemenere gevallen. Eerst wordt bestudeerd of de resultaten van kracht blijven indien gereedschappen verschillende afmetingen hebben. Vervolgens worden problemen met meerdere machines getest. Uit computerexperimenten blijkt dat de voorgestelde ondergrens ook voor deze uitbreidingen erg goed is. Voor 98 procent van de geteste problemen blijkt de ondergrens gelijk aan de optimale oplossing.

In hoofdstuk 5 wordt het job grouping probleem vanuit een andere invalshoek bestudeerd. Doel van het onderzoek is om na te gaan of een aantal lokale zoekmethoden goede oplossingen leveren voor het probleem en of deze oplossingen binnen acceptabele rekentijd worden bereikt. Lokale zoekmethoden onderscheiden zich van de bovengenoemde methoden in de wijze, waarop een oplossing wordt gevonden. De oplossing wordt niet stapsgewijs opgebouwd, maar uitgaande van een (random) oplossing worden 
nieuwe oplossingen gegenereerd en onderzocht. Een oplossing van het probleem bestaat uit een verdeling van de opdrachten in een aantal groepen. Een nieuwe oplossing kan worden verkregen door bepaalde opdrachten van de ene naar de andere groep te verplaatsen of door twee opdrachten in verschillende groepen met elkaar te verwisselen. Iedere oplossing heeft een aantal buuroplossingen. Een doelstellingsfunctie wordt gebruikt om de kwaliteit van de oplossing te beschrijven. Door op een slimme manier de andere oplossingen in de buuromgeving van de huidige oplossing te bekijken, kan een betere oplossing worden verkregen, dat wil zeggen een oplossing, die gebruik maakt van minder uitvoerbare groepen. Soms kan het verstandig zijn om tijdelijk een verslechtering van de doelstellingsfunctie toe te staan. Het verwisselen van twee opdrachten kan een verslechtering betekenen van de doelstellingswaarde, maar het is mogelijk dat na enkele verwisselingen een oplossing wordt bereikt met een lager aantal uitvoerbare groepen. Vier verschillende lokale zoekmethoden, te weten een eenvoudige verbeteringsstrategie, een tabu-zoekmethode, een simulated annealing aanpak en een variable-depth methode zijn onderzocht. Experimenten zijn uitgevoerd om vast te stellen welke methoden efficiënt werken. Tevens wordt de invloed van de startoplossing, de doelstellingsfunctie, de buuromgeving en de stopcriteria onderzocht. Uit de experimenten blijkt dat veel instanties waarvoor de eerder genoemde methoden (in hoofdstuk 3) geen optimale oplossing geven met behulp van lokale zoekmethoden wel optimaal kunnen worden opgelost. De keuze van de startoplossing, de doelstellingsfunctie en de buuromgeving blijkt van veel sterkere invloed dan de keuze van de methode zelf, indien tijdens het proces verslechteringen van de doelstellingsfunctie worden toegestaan. In een aantal gevallen is de tijd om een optimale oplossing te bereiken echter aanzienlijk.

In hoofdstuk 6 wordt een ander tool management probleem, het tool switching probleem, bestudeerd. De doelstelling is het vinden van een opdrachtenvolgorde, waarbij het aantal gereedschappen dat moet worden toegevoegd aan het magazijn tijdens de produktie wordt geminimaliseerd. De gereedschappen passen niet tegelijkertijd in het magazijn en het kan noodzakelijk zijn om bepaalde gereedschappen een aantal keren toe te voegen en te verwijderen (omdat andere gereedschappen op dat moment noodzakelijk zijn). Niet het aantal keren dat de machine moet worden omgesteld (zoals in het job grouping probleem), maar het aantal gereedschappen dat wordt gewisseld is hier van belang. Het tool switching probleem is vooral relevant indien de tijd, die nodig is voor het verwisselen van een gereedschap, aanzienlijk is vergeleken met de produktietijd voor de opdracht (of serie opdrachten), zoals dit voorkomt in bepaalde sectoren van de metaalindustrie. In dit hoofdstuk wordt het tool switching probleem zowel vanuit theoretisch oogpunt als met behulp van experimenten bestudeerd. Er wordt bewezen dat 
het probleem al NP-moeilijk is indien het magazijn slechts twee gereedschappen bevat. Een nieuw bewijs wordt gegeven voor het feit dat gegeven een vaste opdrachtenvolgorde binnen polynomiale tijd een optimale toewijzing van de gereedschappen kan plaatsvinden. Nadruk wordt gelegd op de overeenkomsten met andere bekende problemen uit de combinatorische optimalisering (het handelsreizigersprobleem, het blok minimalisatieprobleem, het interval matrix herkenningsprobleem, etc.) en heuristieken worden voorgesteld, die gebruik maken van deze eigenschappen. De resultaten van computerexperimenten met heuristische oplossingsmethoden en enkele lokale zoekmethoden worden gepresenteerd. 


\section{Curriculum vitae}

Alwin G. Oerlemans, geboren 13 februari 1965 te Utrecht, heeft in 1983 het Voorbereidend Wetenschappelijk Onderwijs aan het College Blaucapel te Utrecht met goed gevolg afgesloten. Van 1983 tot 1988 studeerde hij Econometrie aan de Erasmus Universiteit in Rotterdam. In 1987 was hij werkzaam bij de verzekeringsmaatschappij Nationale Nederlanden N.V. te Rotterdam als assistent-onderzoeker ten behoeve van onderzoek op het gebied van ziektekostenverzekeringen. In 1988 werd aan de Erasmus Universiteit het doctoraal examen Econometrie afgelegd in de richting bedrijfseconometrie. Van 1988 tot 1992 was de auteur verbonden aan de vakgroep Kwantitatieve Economie van de Faculteit der Economische Wetenschappen van de Rijksuniversiteit Limburg te Maastricht, eerst in de functie van assistent in opleiding en in 1992 als toegevoegd docent mathematische besliskunde.

Alwin G. Oerlemans (born February 13, 1965 in Utrecht) studied Econometrics and Operations Research at Erasmus University Rotterdam from 1983 to 1988. In 1987 he worked as research assistant at the insurance company Nationale-Nederlanden N.V. in Rotterdam. In 1988, he received his Master's degree in Econometrics and Operations Research from Erasmus University Rotterdam. Between 1988 and 1992 he held positions as research assistant and lecturer in Operations Research at the Department of Quantitative Economics of the Faculty of Economics and Business Administration of the University of Limburg in Maastricht. During this period, he completed his work on this thesis. 
Production planning is essential for the efficient operation of modern manufacturing systems. The focus of this study is on mathematical programming models for production planning problems arising in flexible manufacturing systems (FMS), and on their solution.

The main part of the book is devoted to a discussion of a number of production planning problems. First, a case study is presented. A hierarchical solution procedure is developed for a real-world production planning problem in the automated manufacturing of printed circuit boards. Special attention goes to the combinatorial modelling of many of the problems which constitute the planning hierarchy. An important aspect of the efficient use of an FMS is the planning of the flow of parts and tools through the production facility. To take advantage of the new opportunities offered by FMS-technology tool management has to be handled well. The simultaneous scheduling of tools and parts adds complexity to the already difficult scheduling operation. Tool management tries to overcome these problems. Two specific tool management problems are studied in detail. Both problems concern the efficient batching and sequencing of parts and tools under different technological constraints. Their objective is to decrease the number of machine-setups. A batching problem with applications in printed circuit manufacturing as well as the metal working industry is studied. The objective is to partition a set of jobs in a minimal number of batches. Solution procedures and lower bounds on the solution value for problems of this type are developed. Under different technological constraints, sequencing of individual operations becomes more important than batching.

Computational experiments show that satisfactory solutions can be obtained for both tool management problems discussed here.

The author received a Master's degree in Econometrics and Operations Research from Erasmus University Rotterdam in 1988. He has held positions as research assistant at Nationale Nederlanden N.V., a major insurance company, and since 1988 as research assistant and lecturer in Operations Research at the Department of Quantitative Economics of the University of Limburg in Maastricht. 